
tert-butyl- $N$-methyl-tyrosine, coupled with CSD data: A survey of interactions in crystal structures of $\mathrm{Fmoc}$-amino acids.

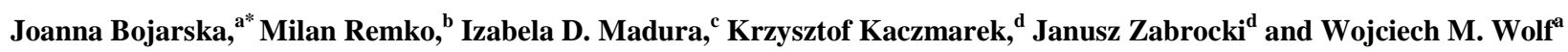

${ }^{a}$ Institute of General and Ecological Chemistry, Lodz University of Technology, Faculty of Chemistry, Żeromskiego 116, Lodz 90-924, Poland, ${ }^{\mathrm{b}}$ Remedika, Sustekova 1, 85104, Bratislava, Slovakia, ${ }^{\mathrm{c}}$ Warsaw University of Technology, Faculty of Chemistry, Noakowskiego 3, 00-664 Warszawa, Poland, ' Institute of Organic Chemistry, Lodz University of Technology, Faculty of Chemistry, Żeromskiego 116, Lodz 90-924, Poland.

\title{
SUPLEMENTARY MATERIALS
}

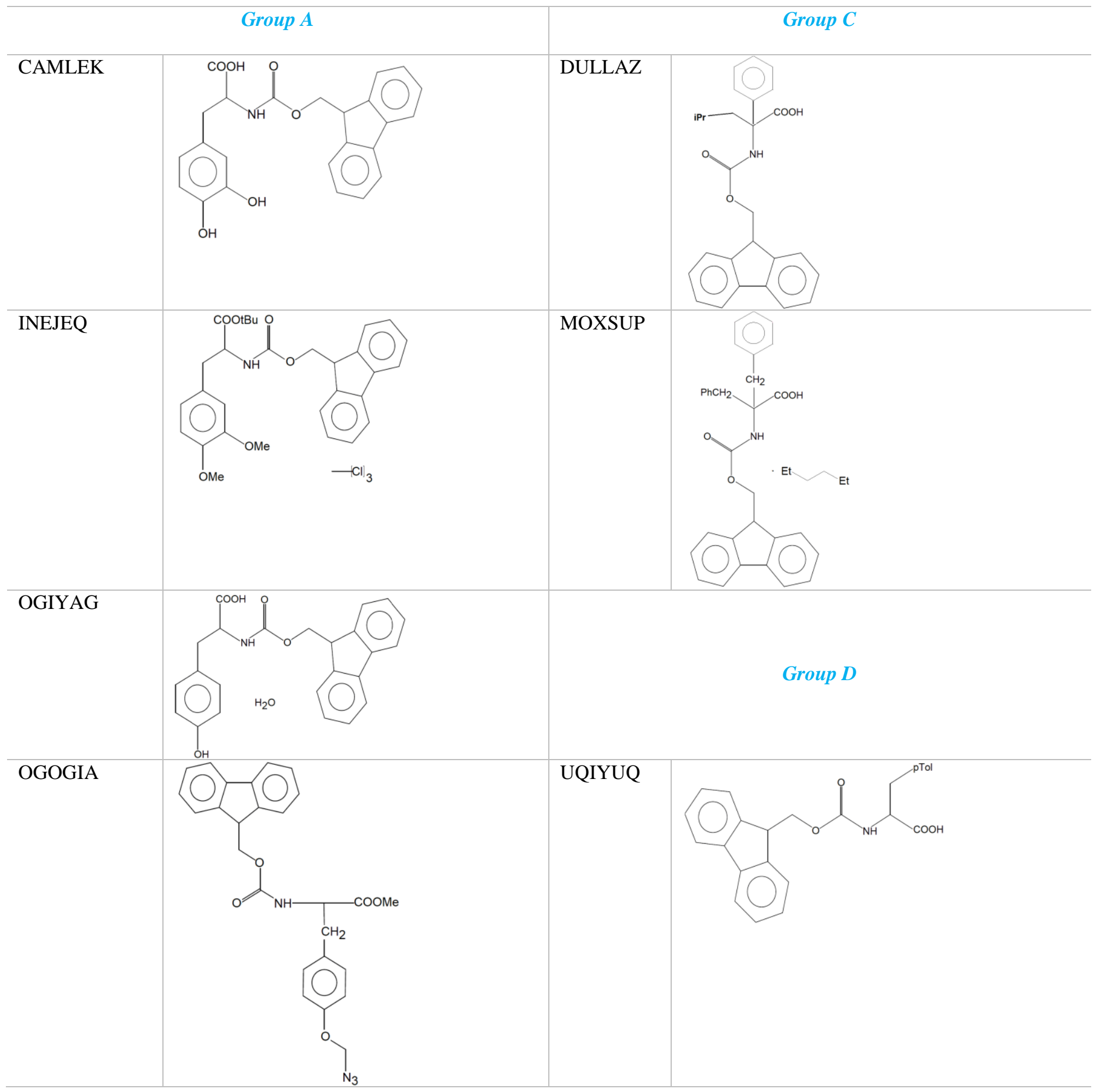


group B

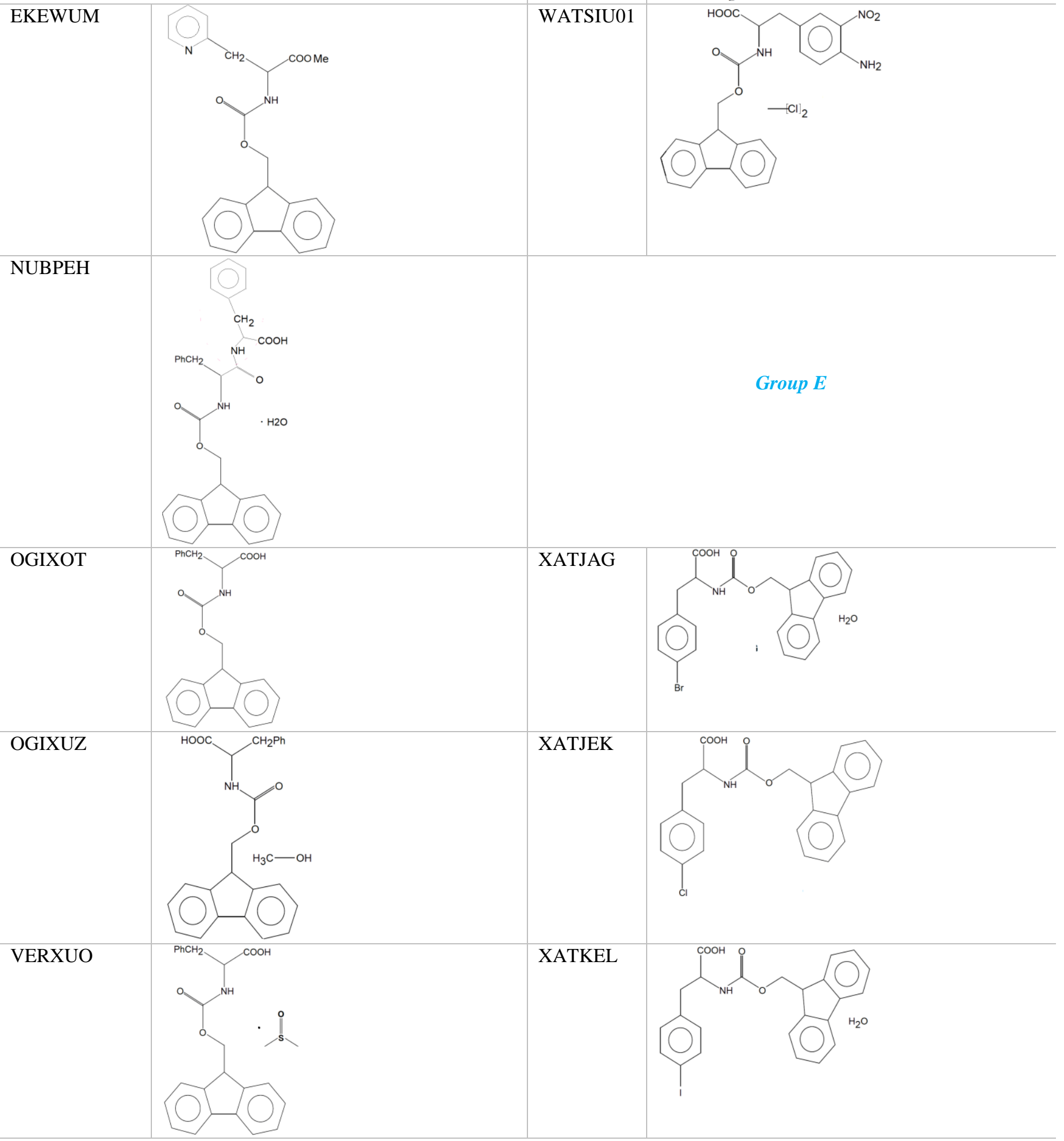




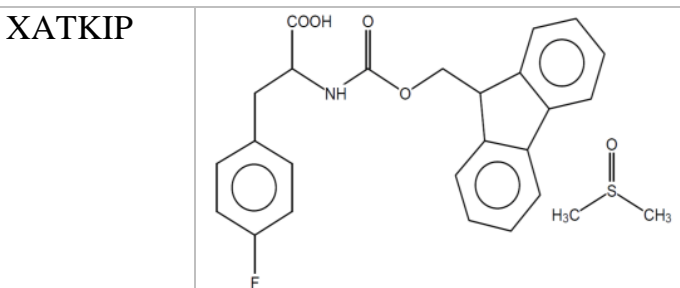

\section{Scheme S1}

Chemical diagram of Fmoc-Tyr/Phe derivatives, retrieved from the CSD.

FMOC-TRYPTOPHAN derivative DIZNIK

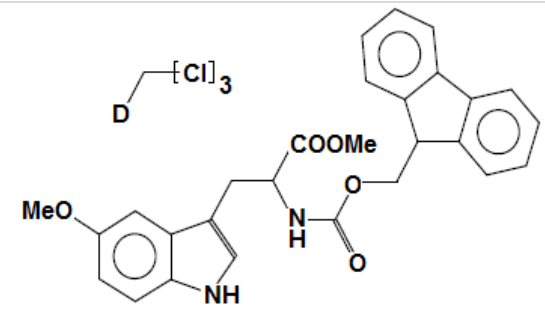

FMOC-ORNITHINE derivative EXOFAY

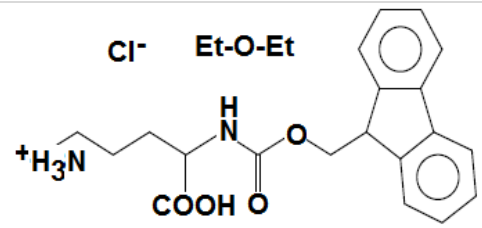

FMOC-LEUCINE derivative

BIZXUE

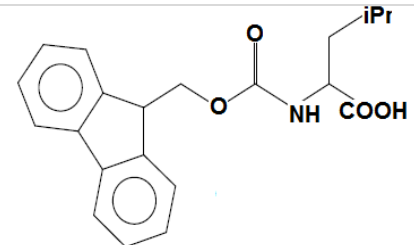

FMOC-ISOLEUCINE derivative QOFHID

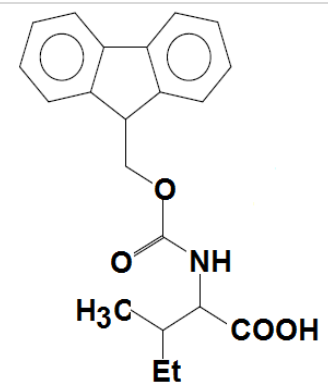

FMOC-SERINE derivative ADAGOE

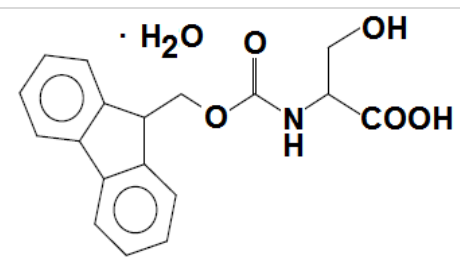

FMOC-GLYCINE derivative

VERQER

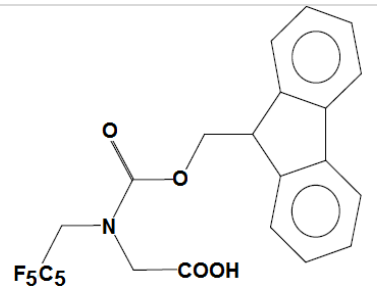

VERQIV

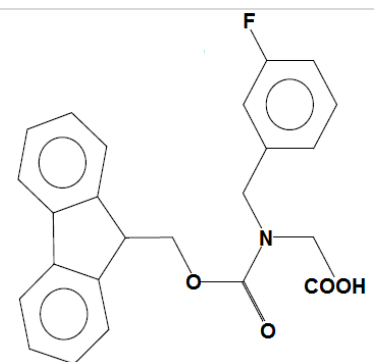

VERQOB

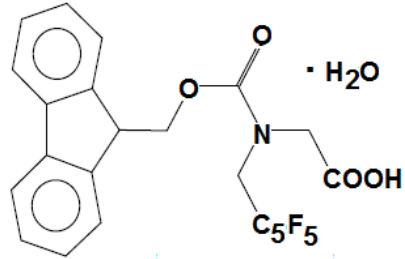

VERXIC

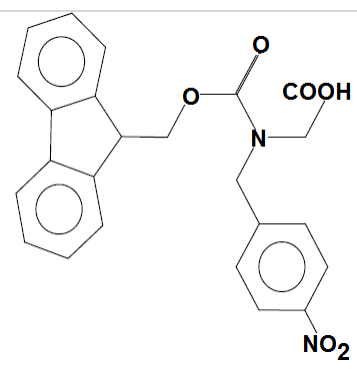

VERXOI

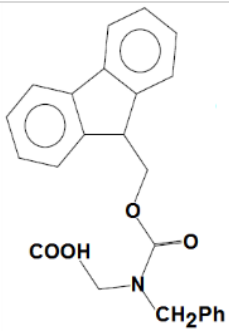


MOHCIW

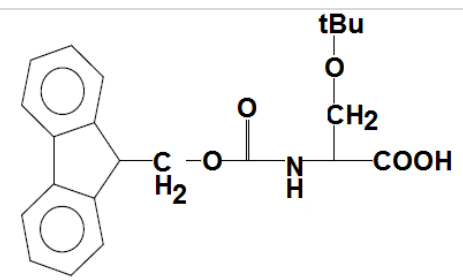

FMOC-ALANINE derivative

ADAGUK

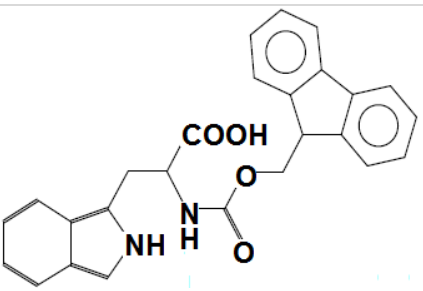

CUWKIO01

CUWKOU01
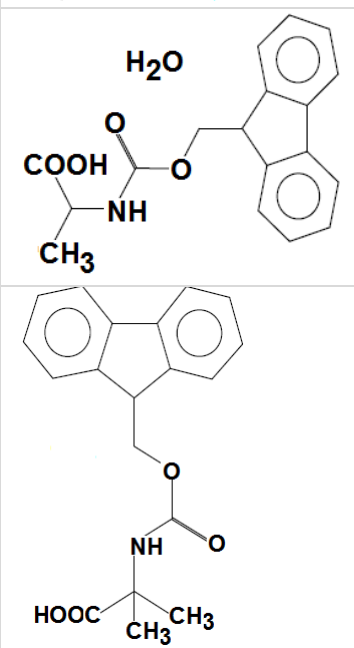

\section{Scheme S2}

Chemical diagram of other Fmoc-AAs, retrieved from the CSD.
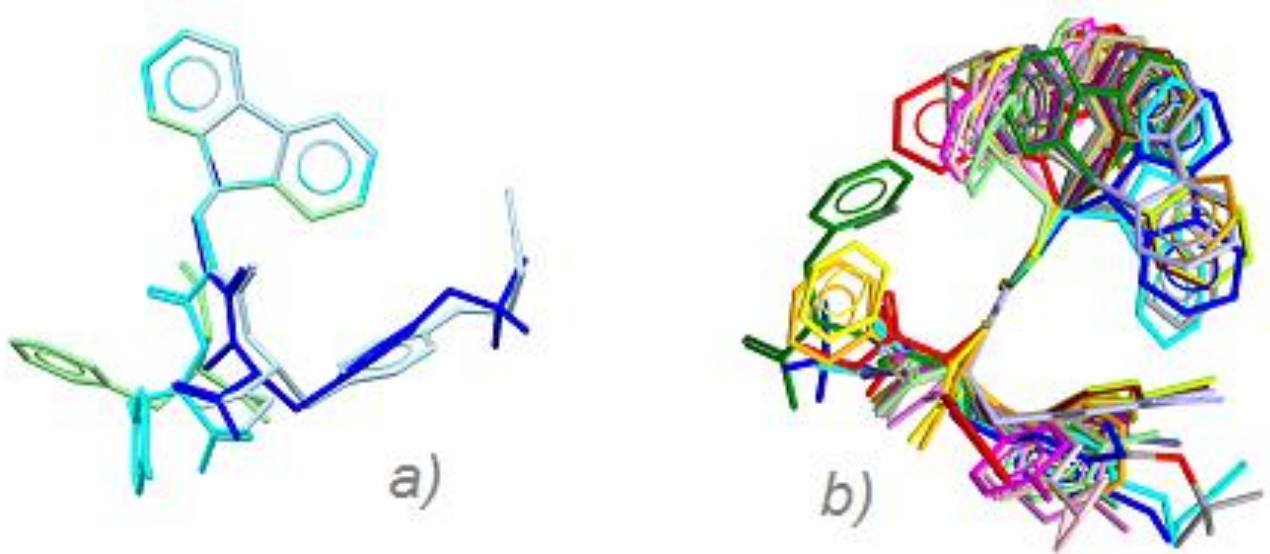

\section{Figure S1}

Superimposition of: $a$ ): (1) (in blue color) and OGIXOT (in light green), OGIYAG (cyan) and OGOGIA (light blue); b): (1): by element; CAMLEK: light green, DULLAZ: yellow; EKEWUM: red, INEJEQ: blue, MOXSUP: orange, NUBPEH: green, OGIYAG: violet, OGIXOT: purple, OGIXUZ: grey, OGOGIA: cyan, UQIYUQ: magenta, UQOGUE: pink, VERXUO: khaki, WATSIU01: light violet, XATJAG: brown, XATJEK: dark violet, XATKEL: limon, XATKIP: beige. 


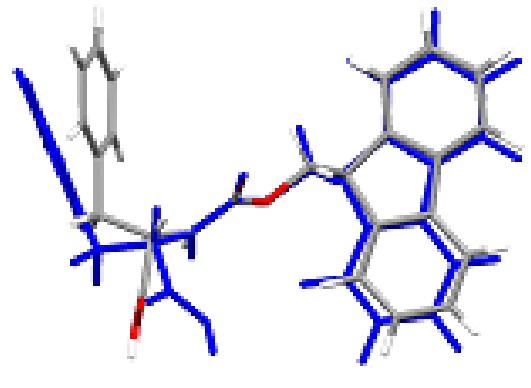

a)

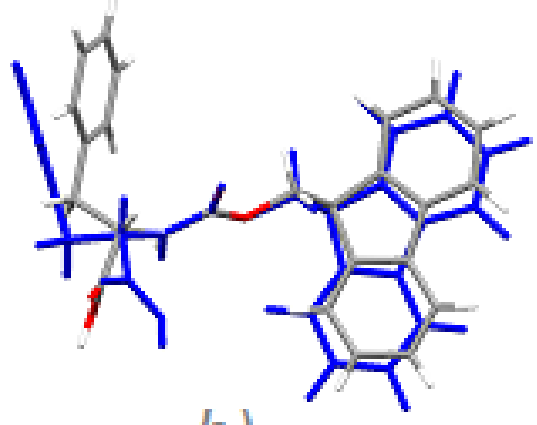

b)

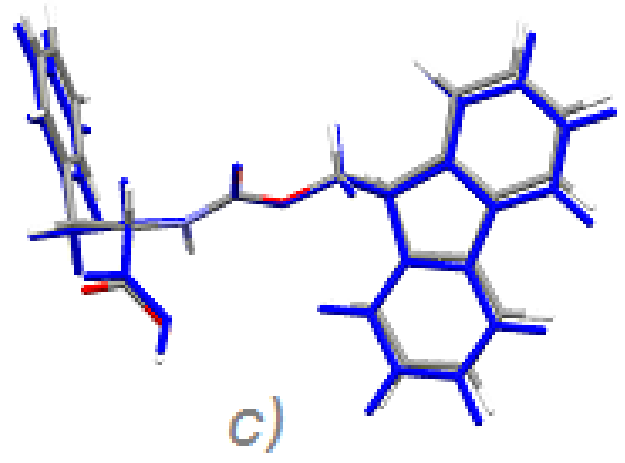

c)

Figure S2

Superposition (with respect to the $\mathrm{O}=\mathrm{C}-\mathrm{N}$ group) of the X-ray structure of OGIXOT and: (a) the B3LYP optimized (blue), (b) B3LYP optimized solvated OGIXOT (blue), and also (c) B3LYP optimized OGIXOT and B3LYP optimized solvated OGIXOT (blue).

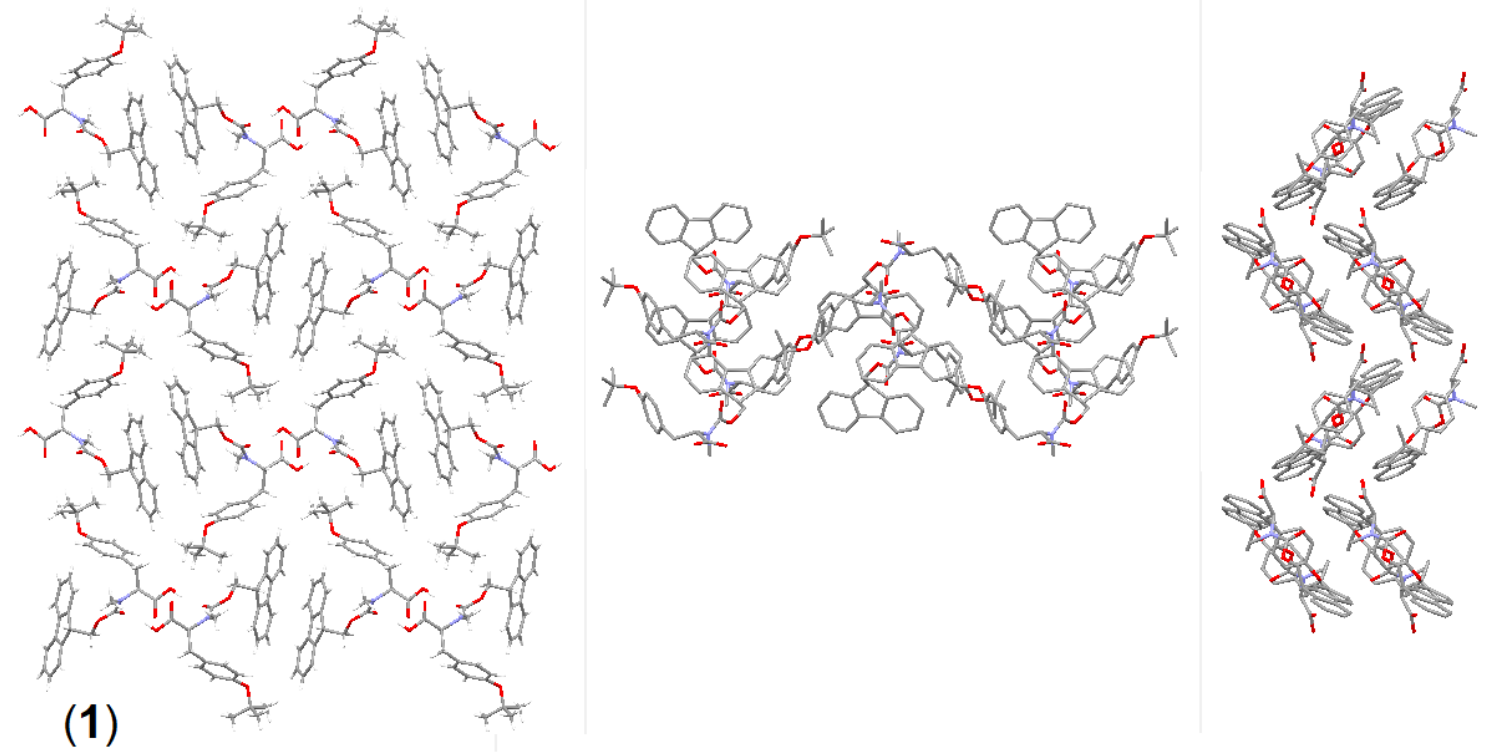

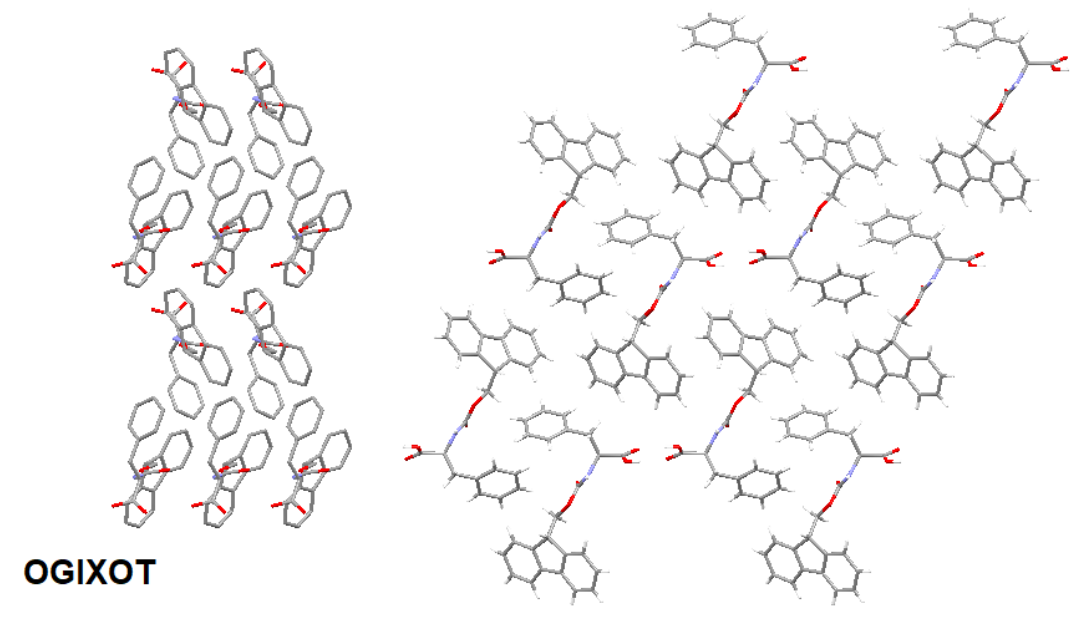

(a)

(b)

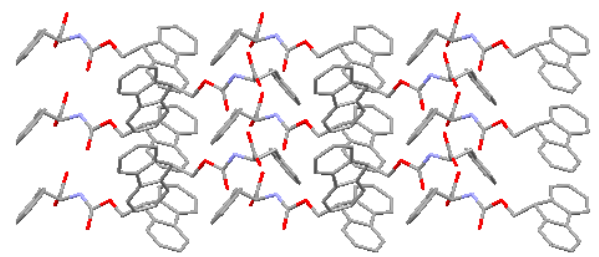

(c)

Figure S3

Crystal packing in the (1) and OGIXOT: views from the $(a) \mathrm{a},(b) \mathrm{b}$, and $(c) \mathrm{c}$ axes. 


\section{OGIXOT}

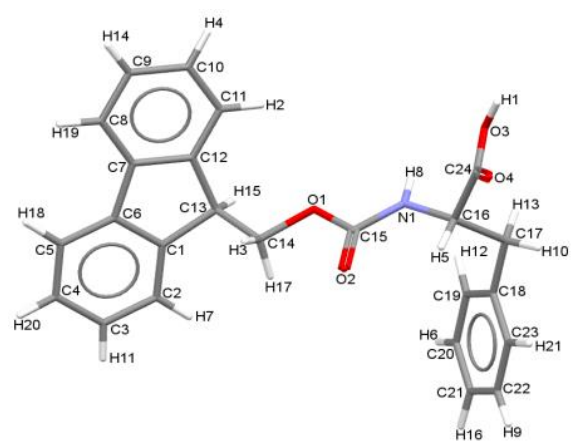

DULLAZ

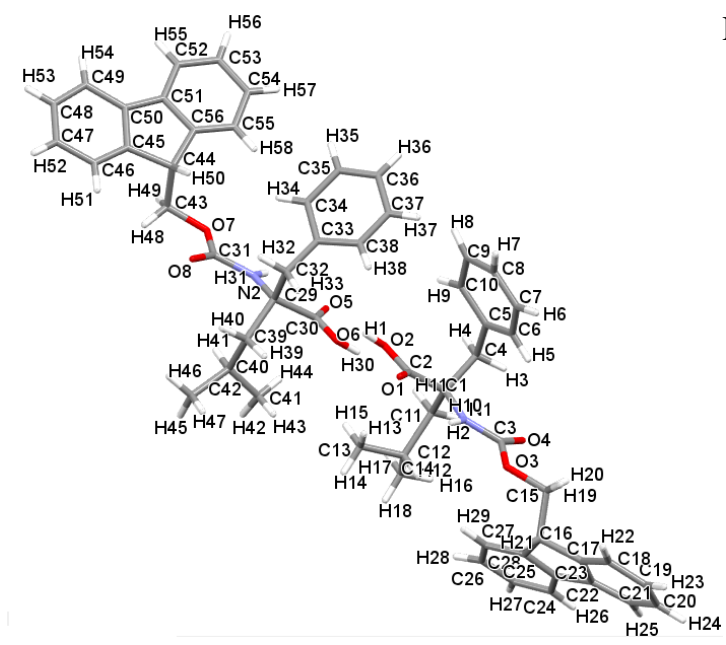

INEJEQ

NUBPEH
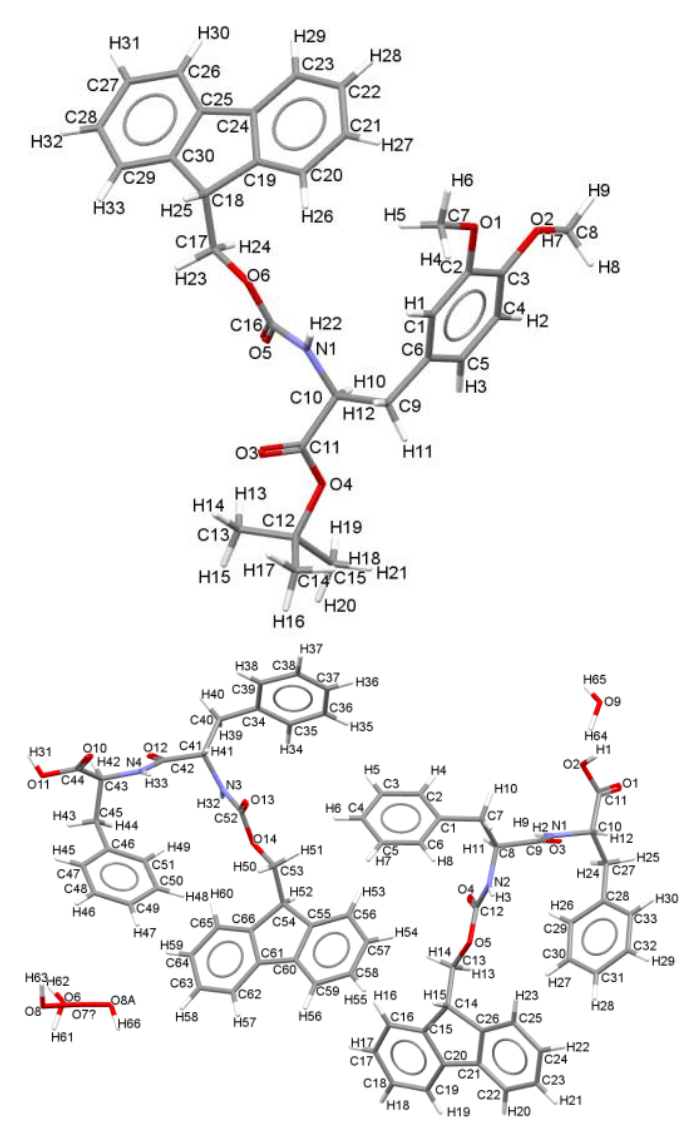

CAMLEK

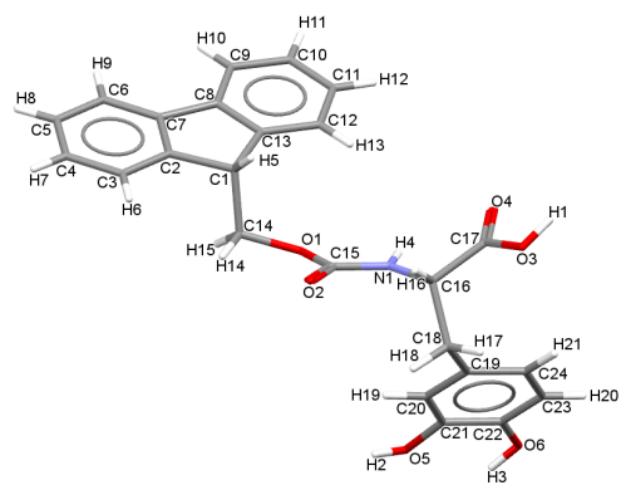

EKEWUM

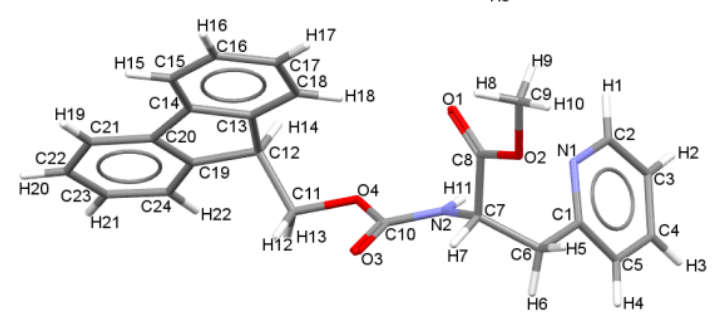

MOXSUP

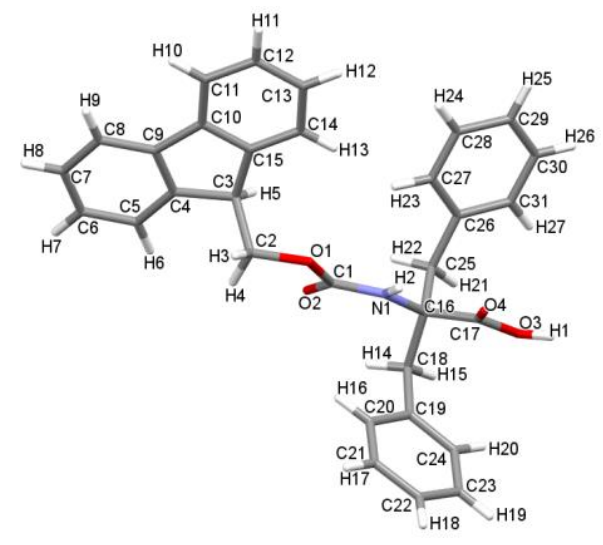

OGIXUZ

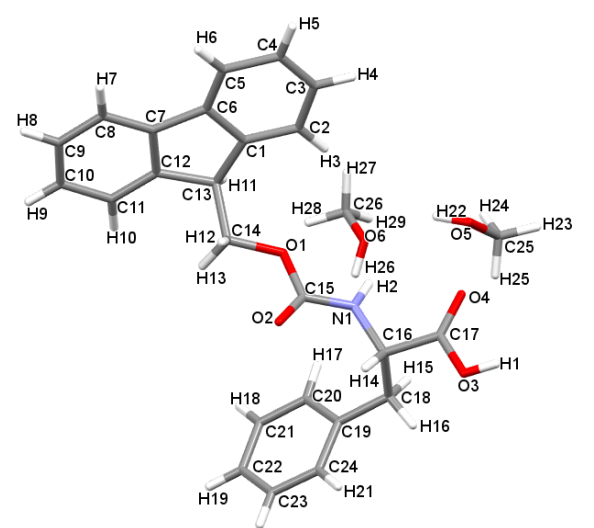


OGIYAG

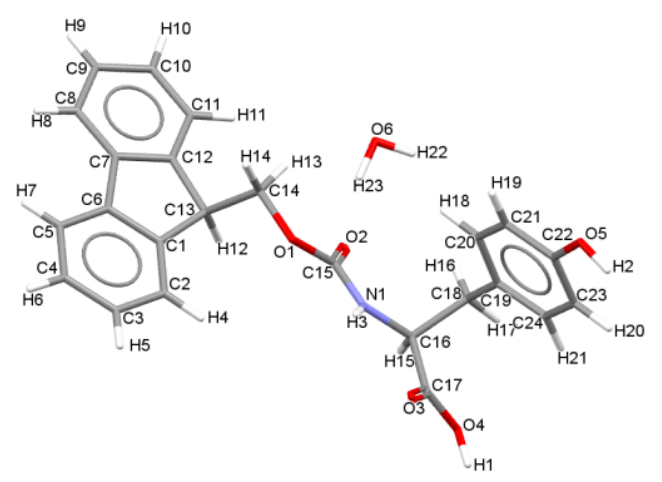

UQIYUQ

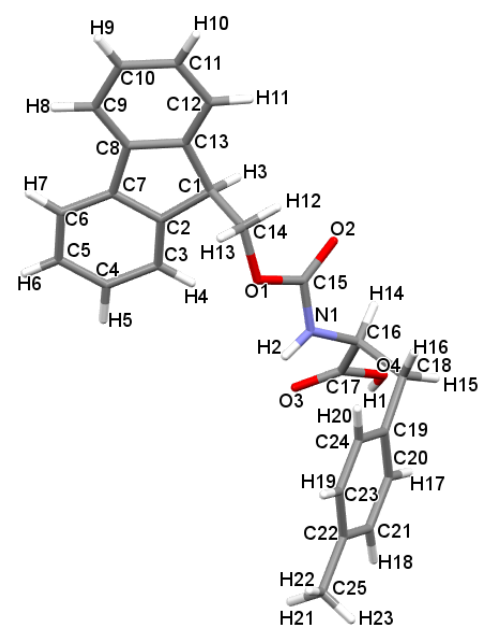

VERXEO

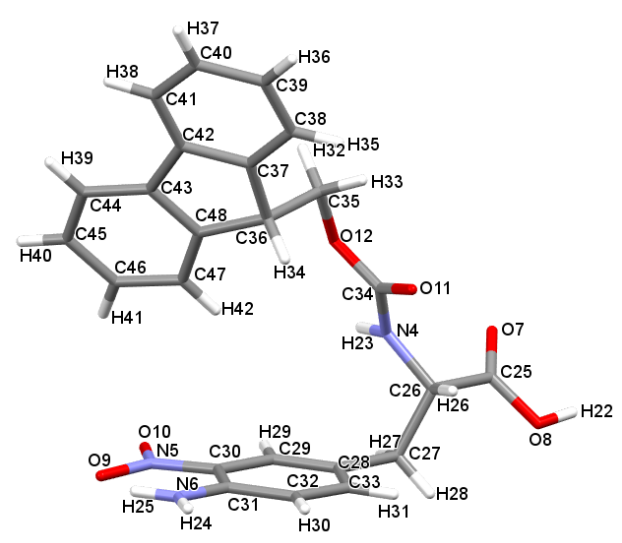

XATJAG

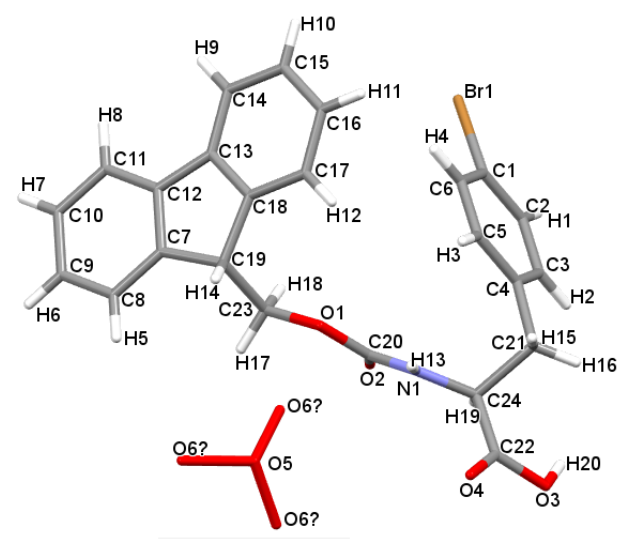

OGOGIA

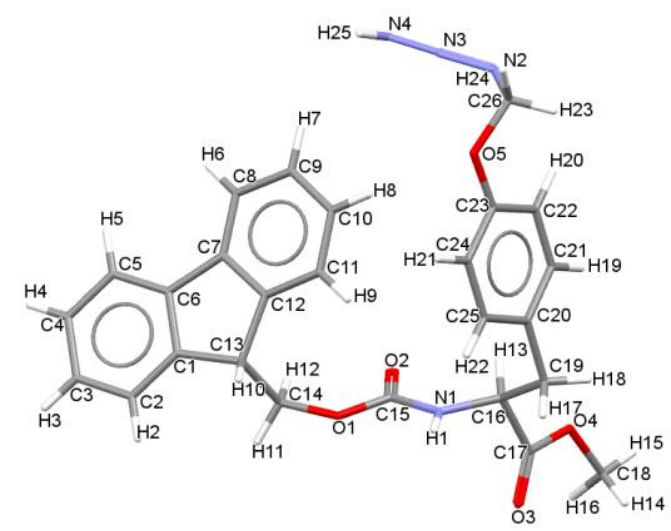

UQOGUE

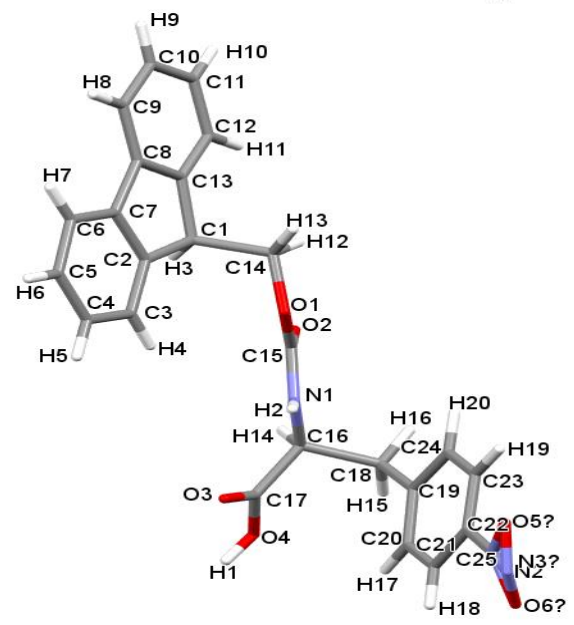

WATSIU01

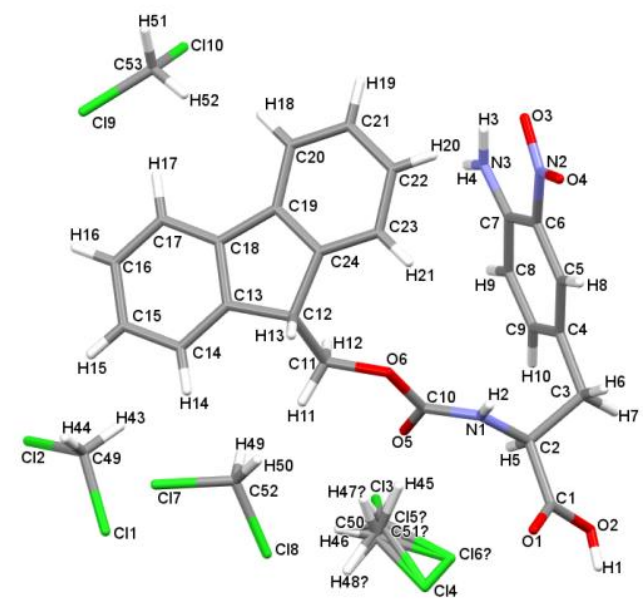

XATJEK

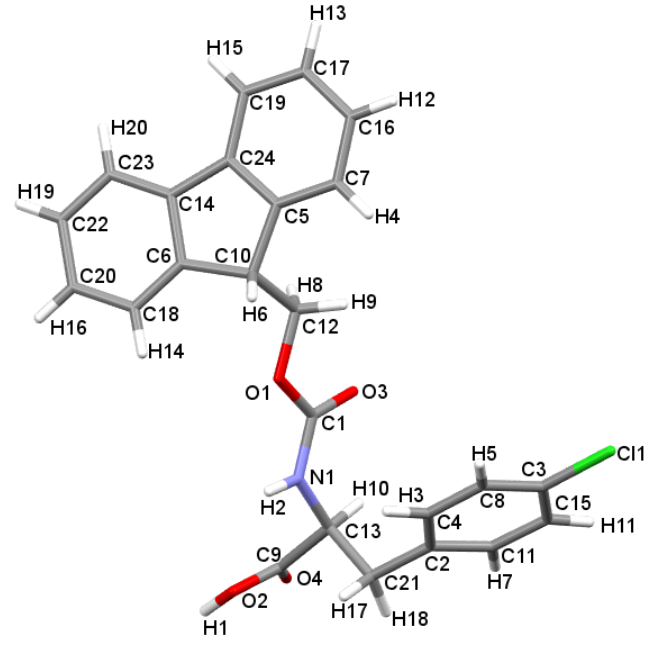


XATKEL

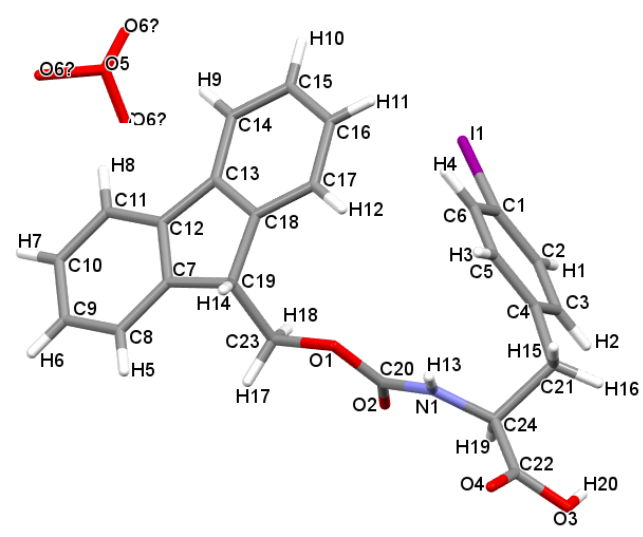

XATKIP

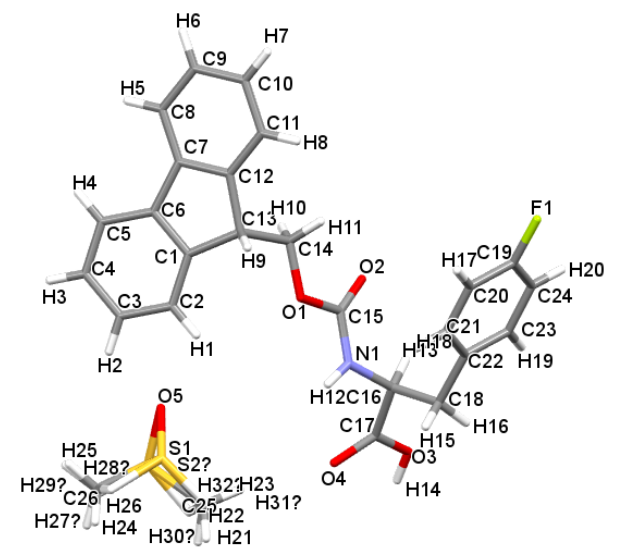

\section{Figure S4}

ORTEP diagrams for Fmoc-Tyr/Phe AAs.

(1)
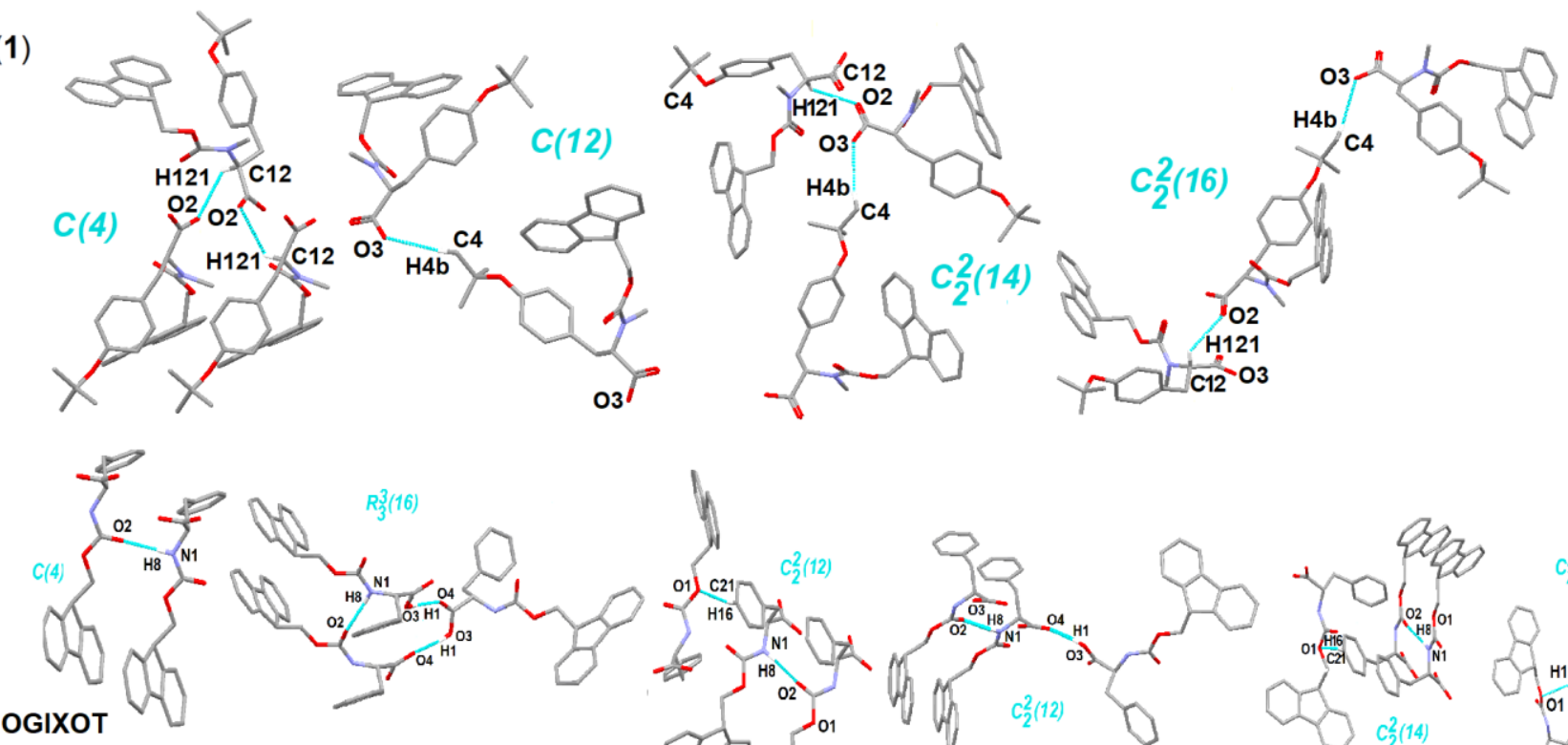

\section{Figure S5}
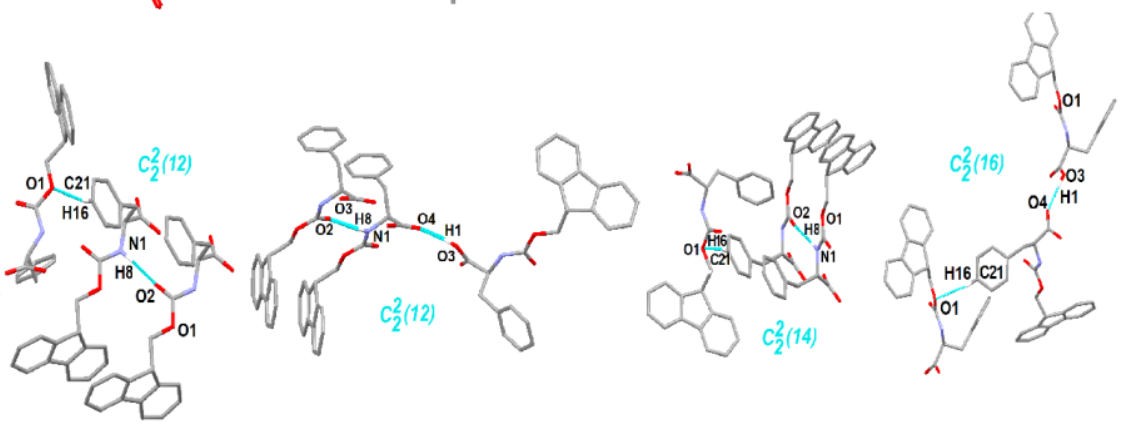

Other supramolecular H-bonding patterns found in (1) and OGIXOT. H-atoms not involved in the H-bonds were omitted for clarity.

$H S d_{\text {norm }}$

ESP

group A

CAMLEK
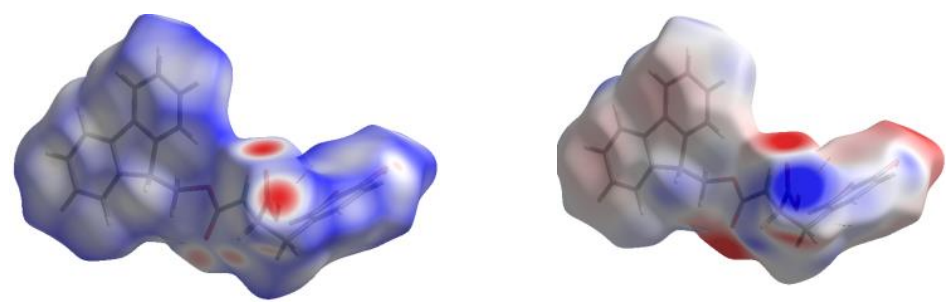
OGOGIA

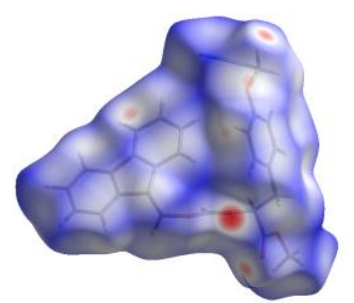

OGIYAG

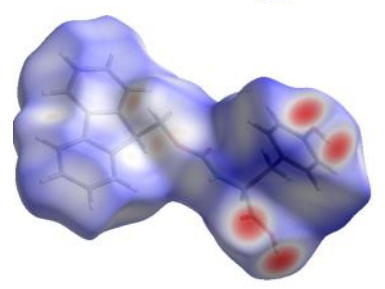

INEJEQ

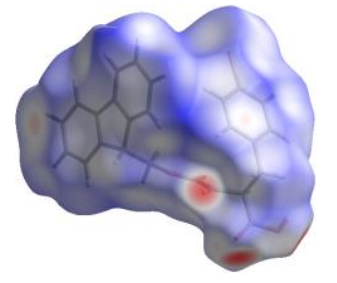

group $\boldsymbol{B}$

VERXUO

OGIXUZ

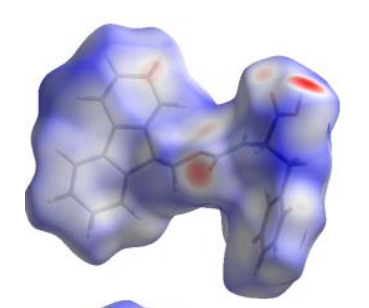

EKEWUM
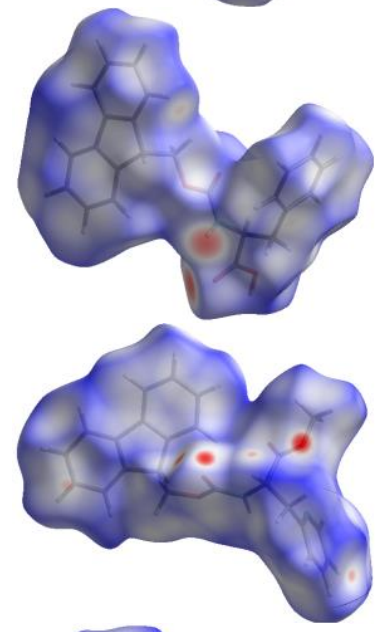

NUBPEH

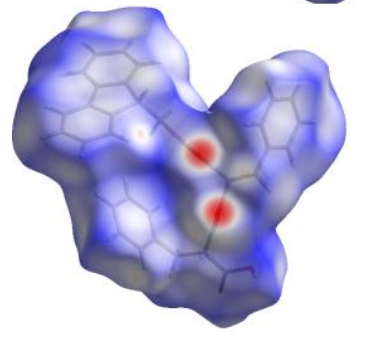

group $\mathbf{C}$ MOXSUP

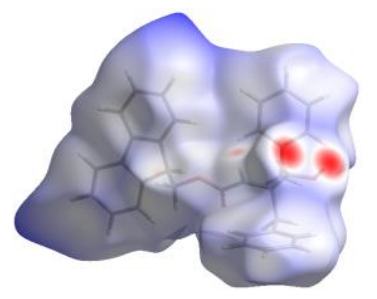

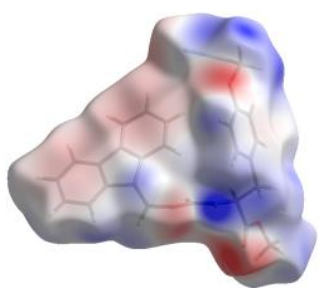
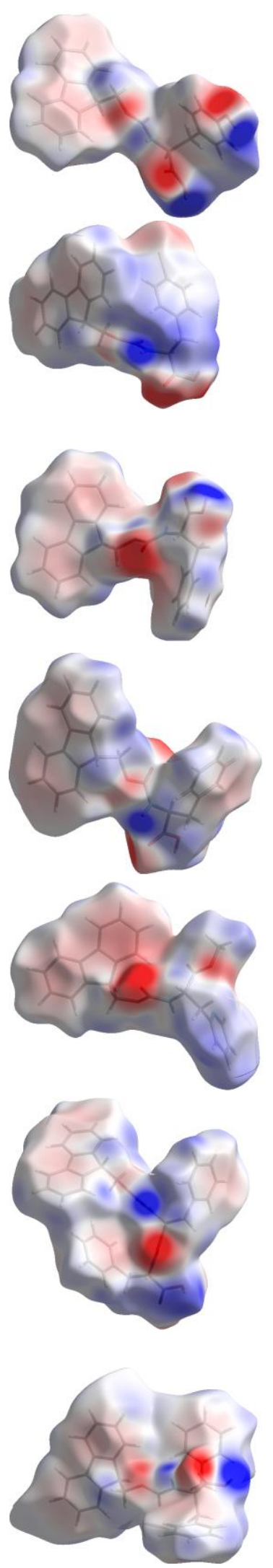


\section{DULLAZ}
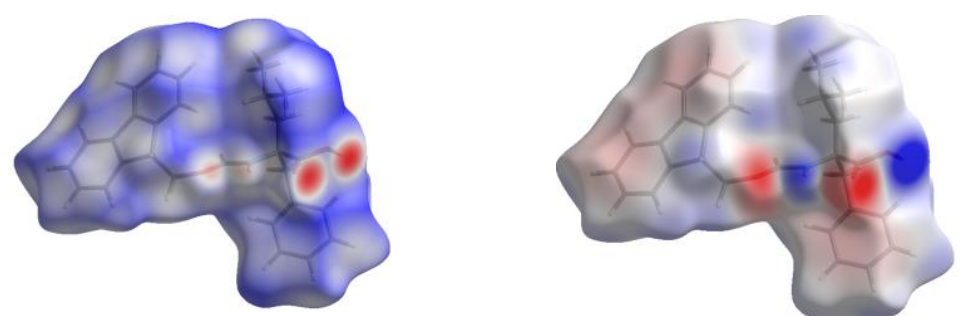

group $D$

WATSIU01

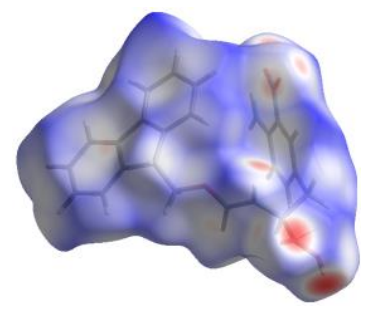

UQOGUE
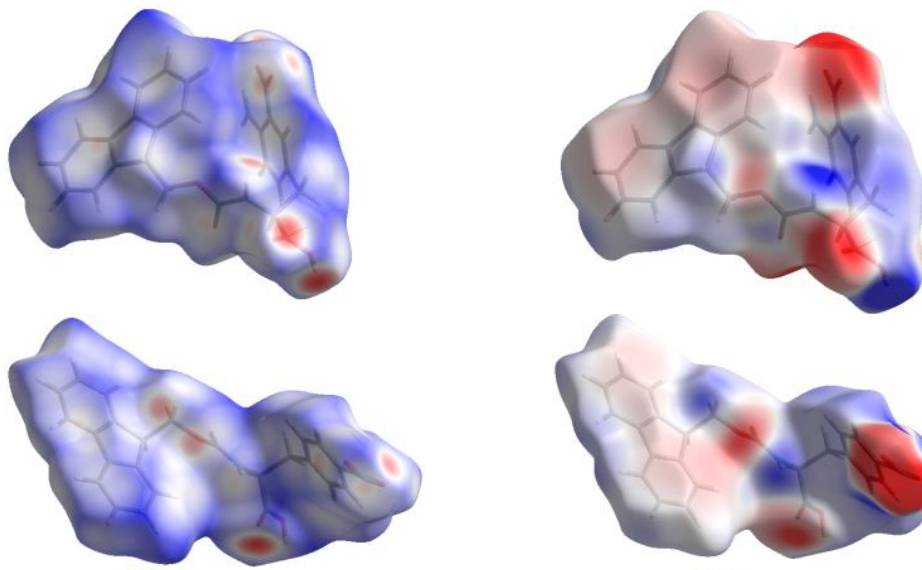

UQIYUQ
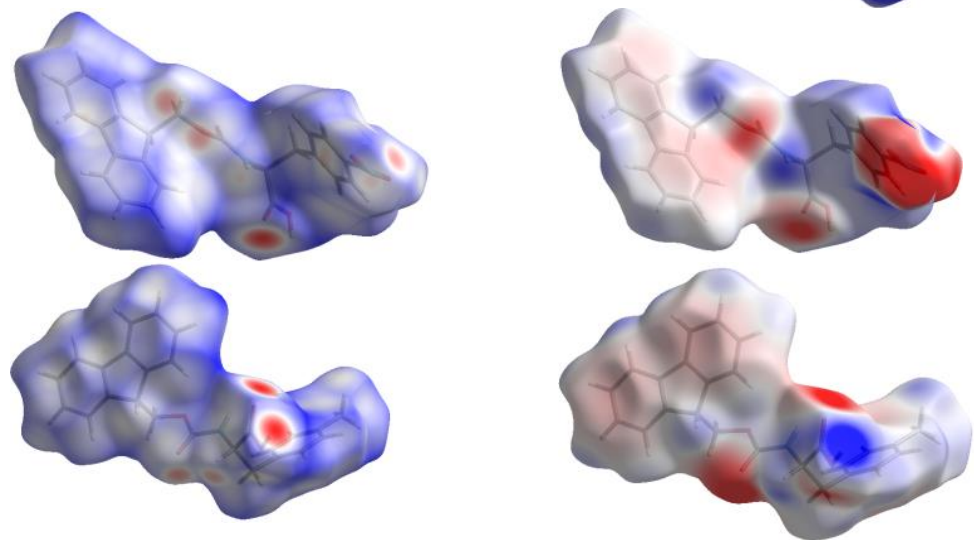

group $E$

XATKEL
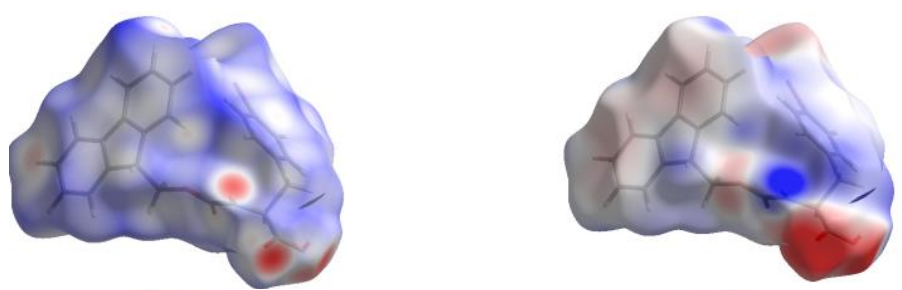

XATJAG
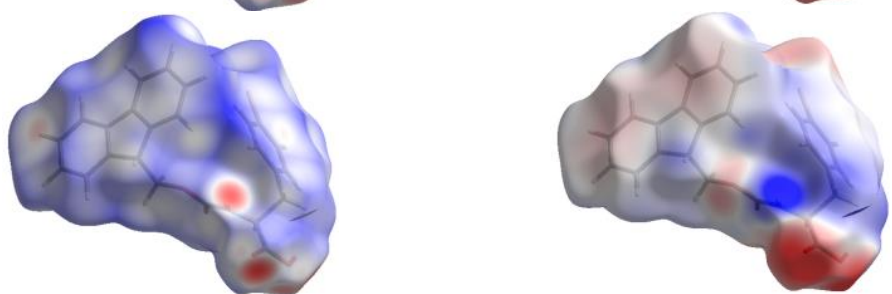

XATJEK

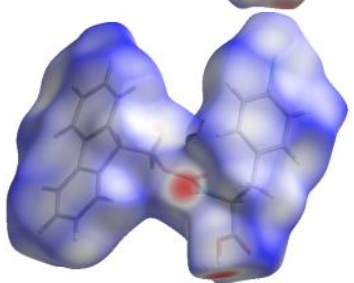

XATKIP
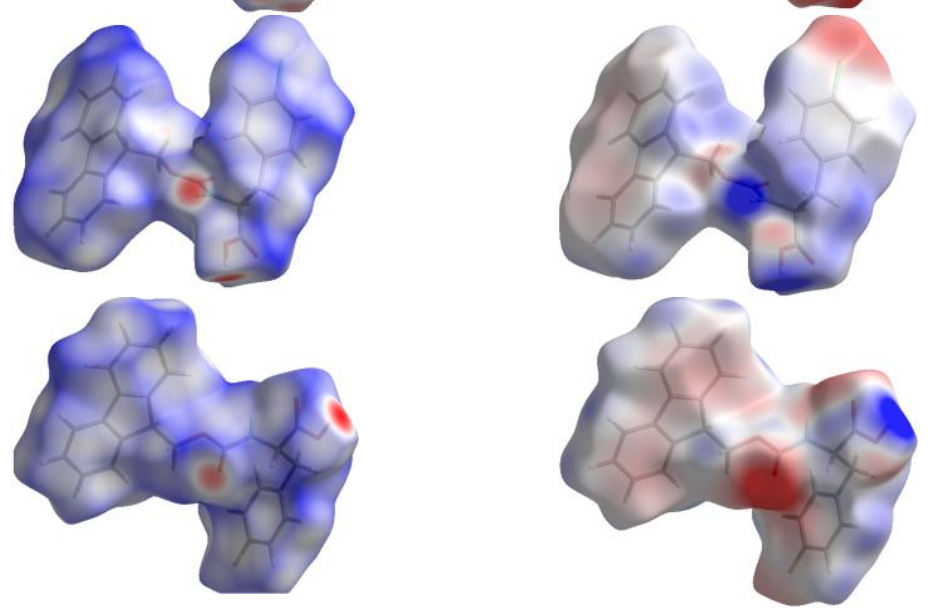

\section{Figure S6}

Views of the HSs with $d_{\text {norm }}$ and ESPs for Fmoc-Tyr/Phe crystals. 


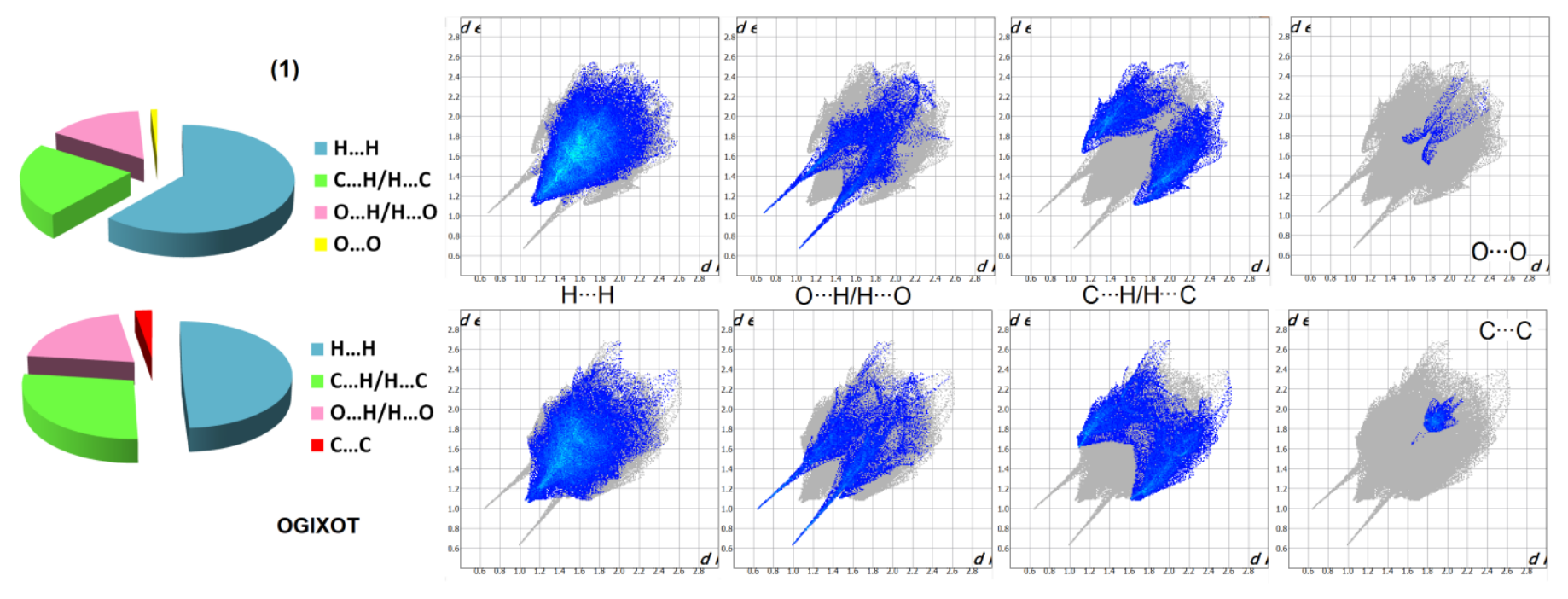

Figure S7

Full and decomposed FPs for (1) OGIXOT.

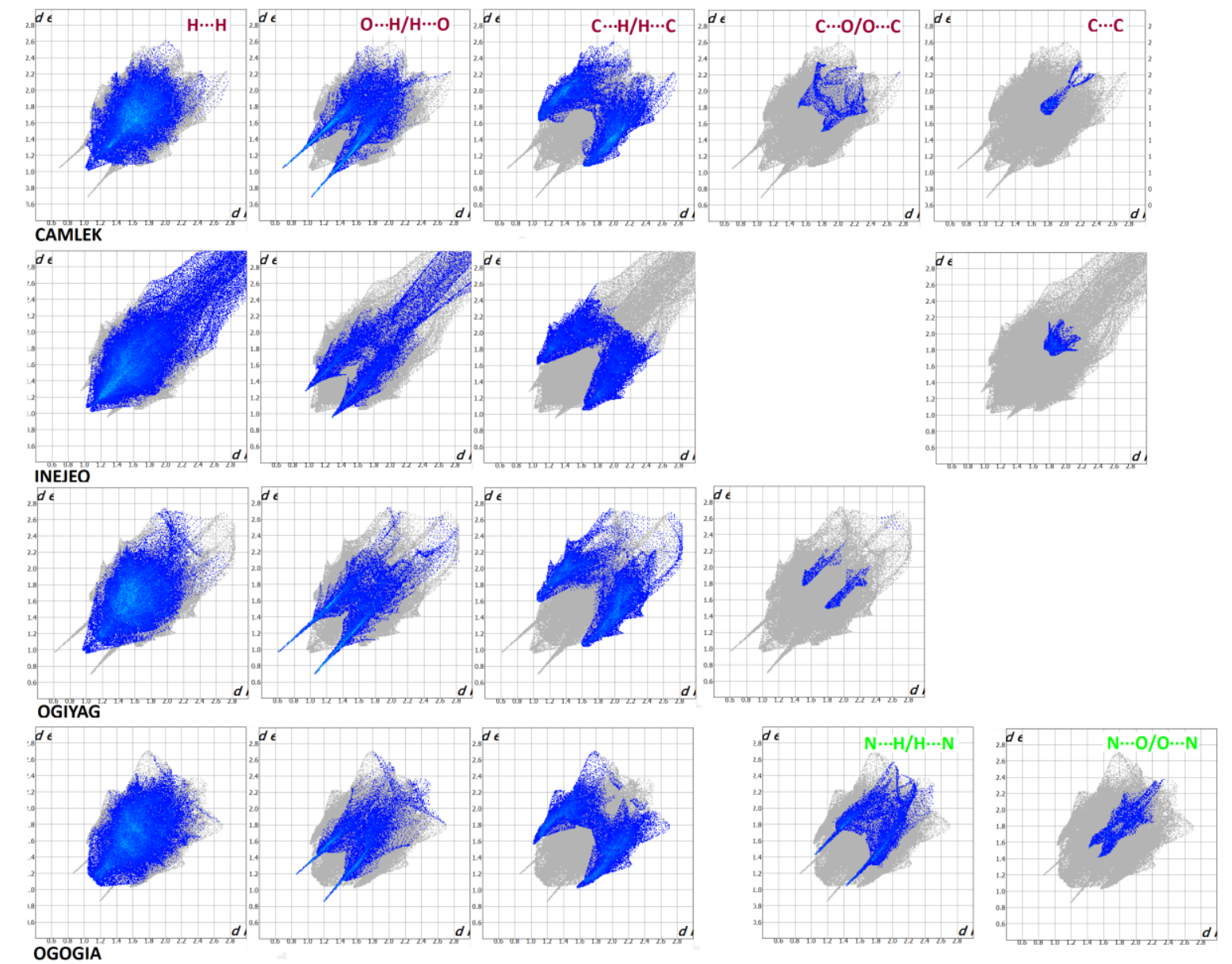

group A 

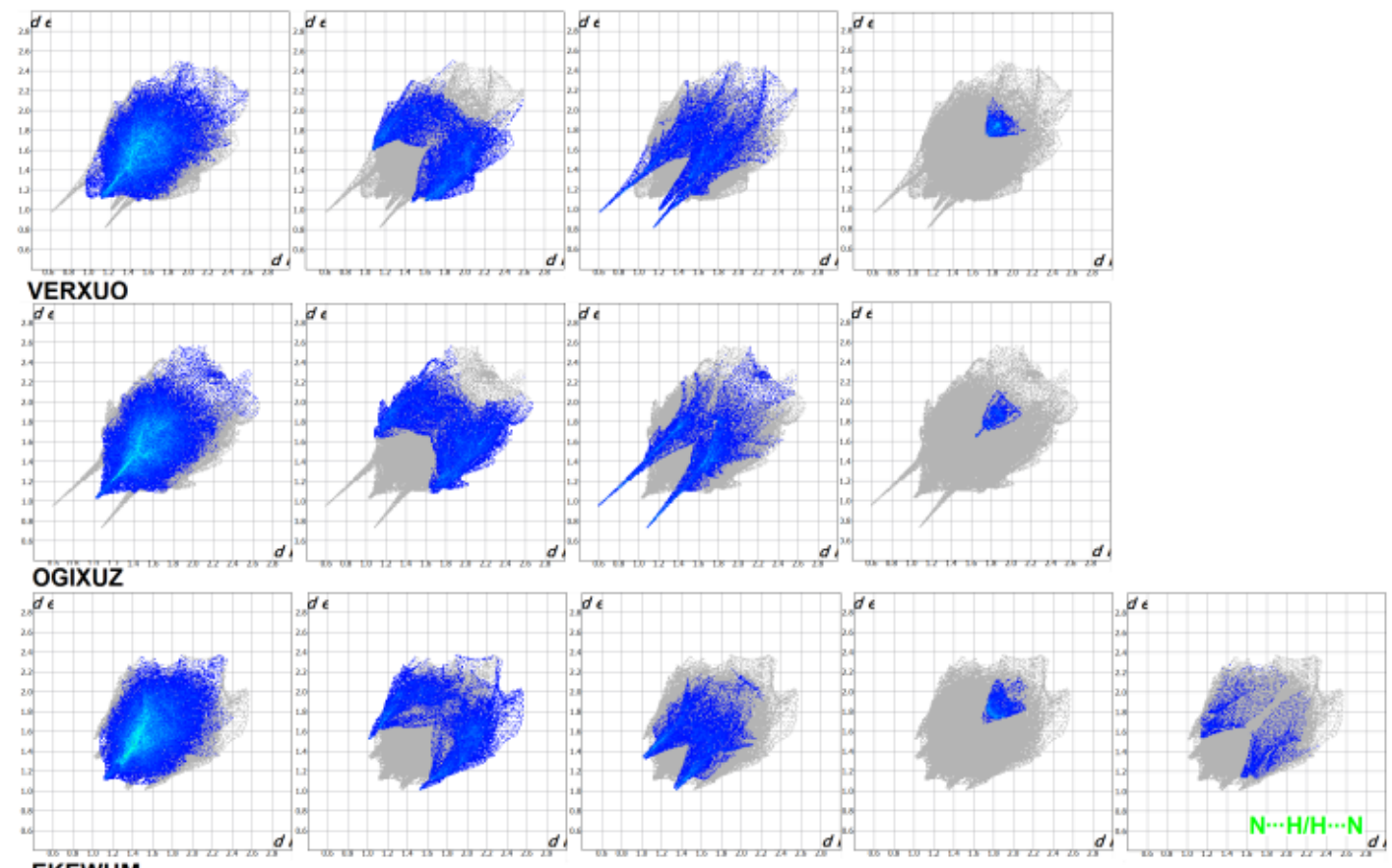

group $\boldsymbol{B}$
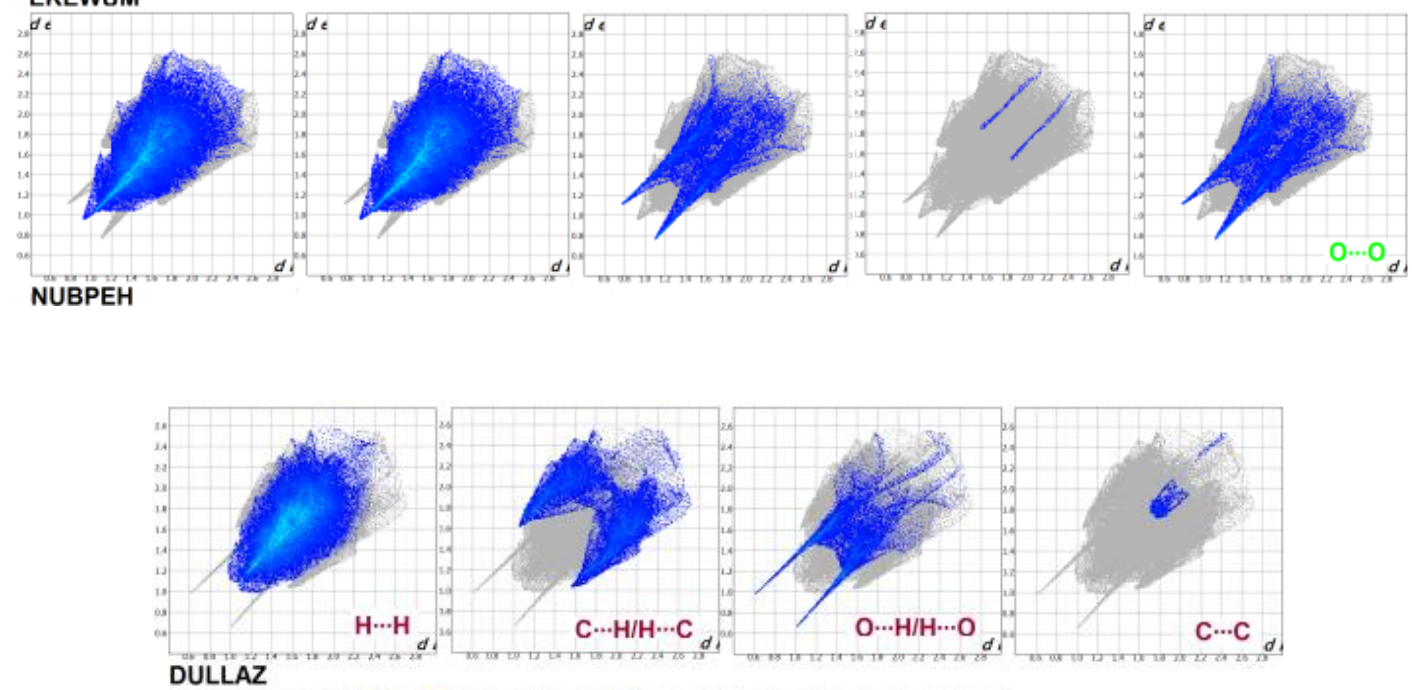

group $C$

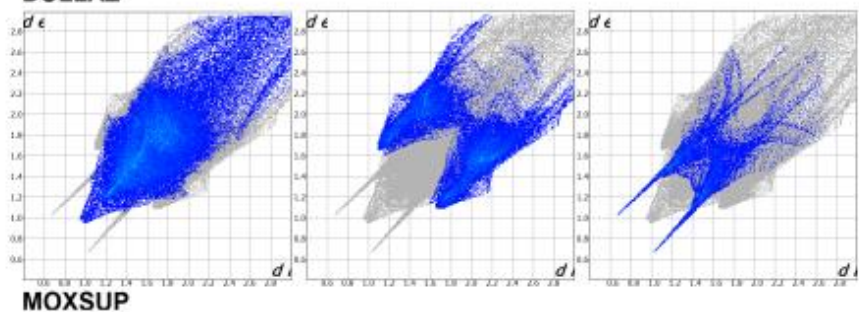



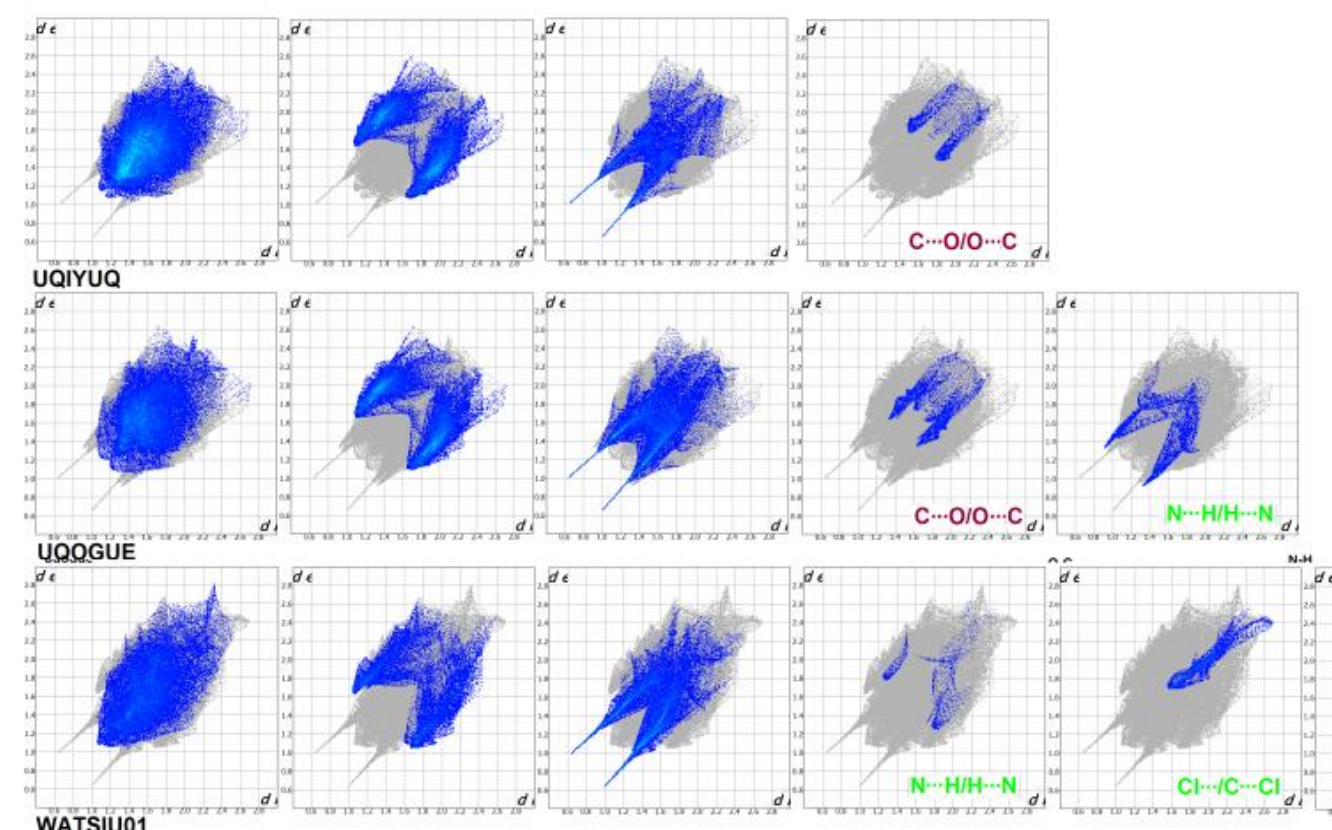

group $D$
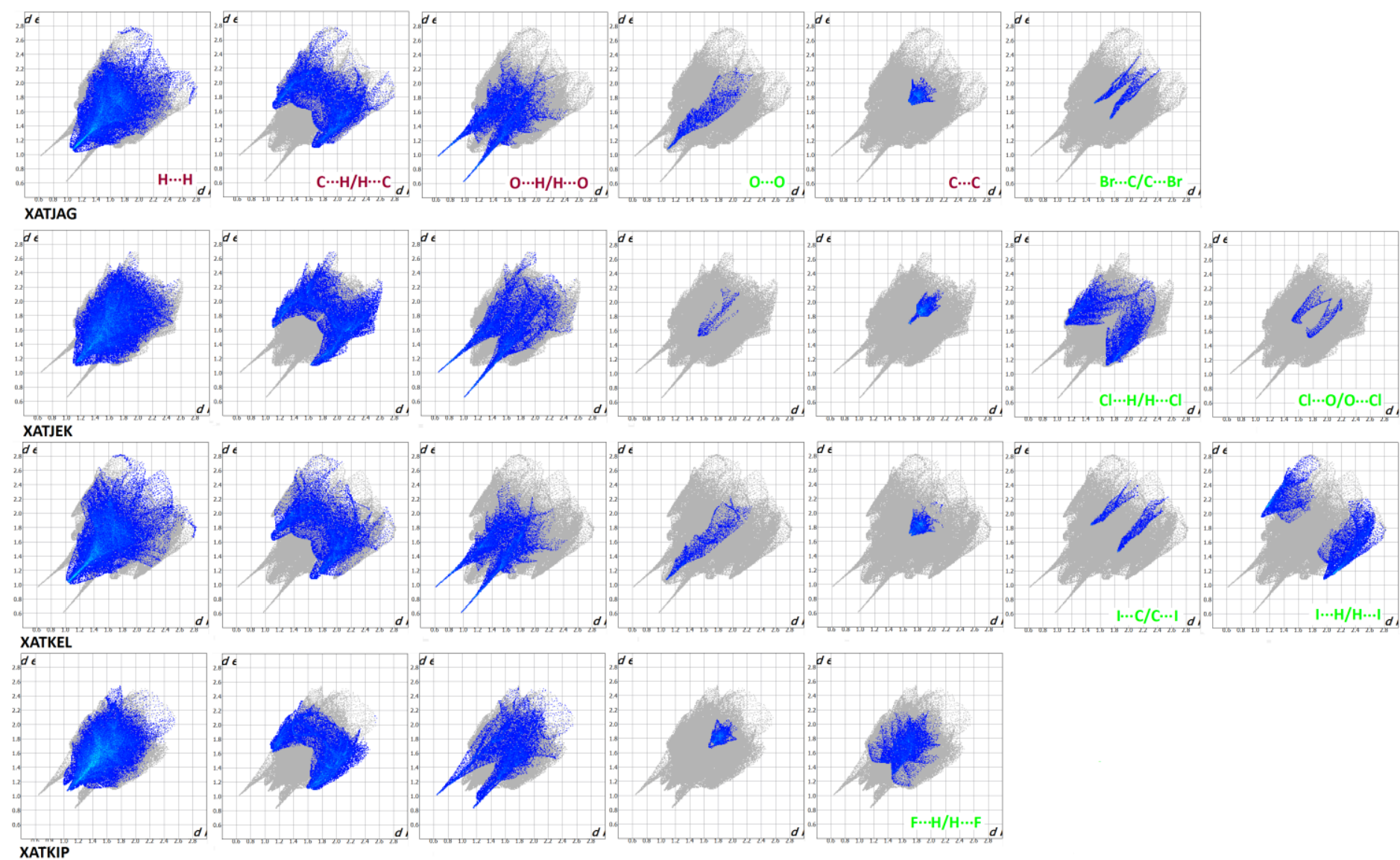

group $E$

Figure S8

Decomposed FPs for Fmoc-Tyr/Phe derivatives. 


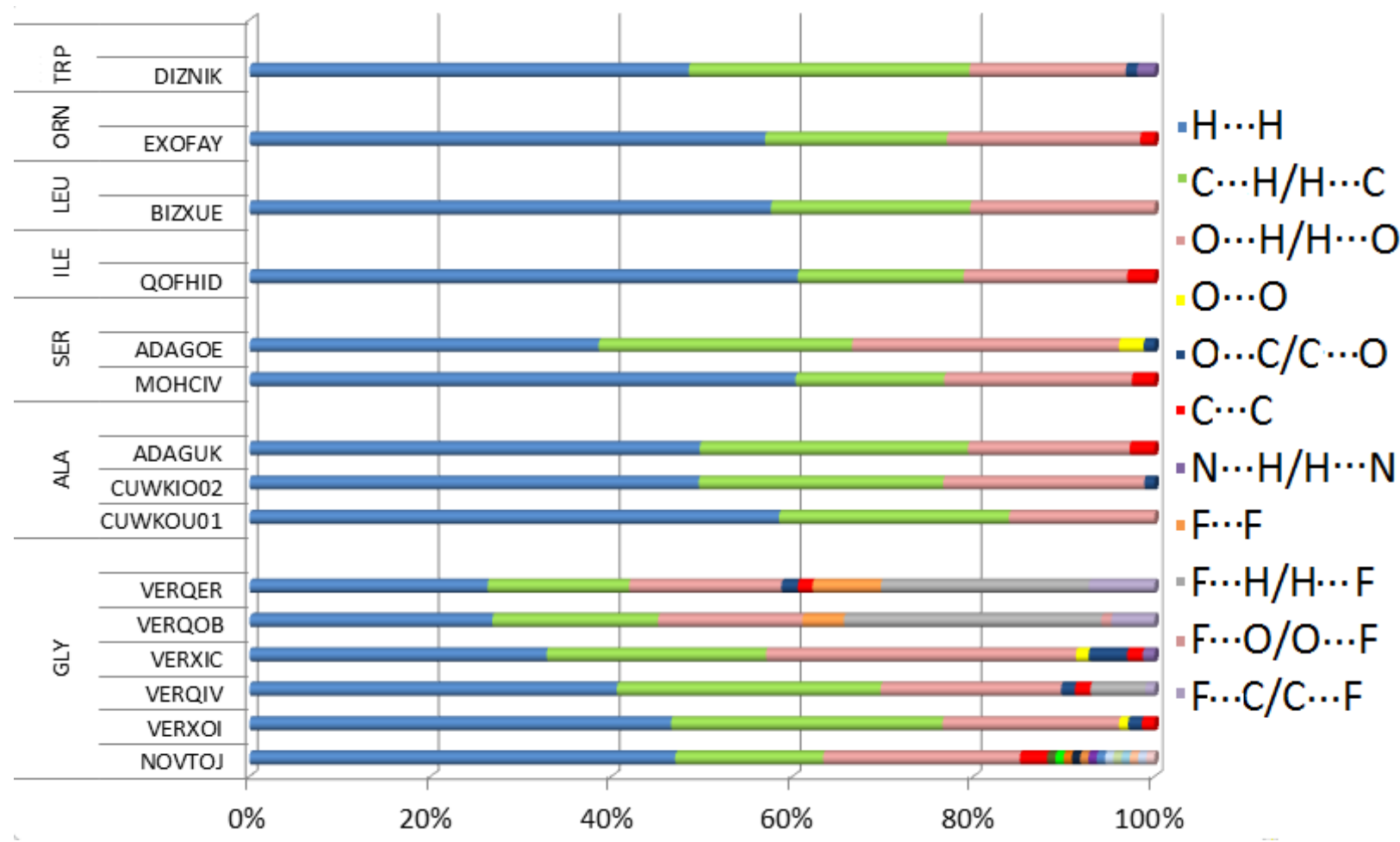

\section{Figure S9}

Percentage conribution of close interactions for other Fmoc-AA derivatives

\section{Table S1}

Name of considered Fmoc-Tyr/Phe derivatives.

CSD code (reference)

\section{Fmoc-TYROSINE (FmocY) / PHENYLALANINE (FmocF) derivatives}

\section{CAMLEK}

9-fluorenylmethoxycarbonyl-3,4-dihydroxyphenylalanine

(Fichman et al., 2016)

\section{DULLAZ}

$N$-((9H-fluoren-9-ylmethoxy)carbonyl)-a-isobutylphenylalanine

(Wang et al., 2015)

\section{EKEWUM}

Methyl 2(S)-(N-fluoren-9-ylmethoxycarbonylamino)-3-(2-pyridyl) propionate

(Clegg \& Horsburgh, 2003)

\section{INEJEQ}

(Clegg \& Elsegood,

\section{MOXSUP}

(Scroggs et al., 2015)

\section{NUBPEH}

(Raeburn et al., 2015)

OGIXOT
(Draper et al., 2015)

OGIXUZ

(Draper et al., 2015)

OGIYAG

(Draper et al., 2015)

\section{OGOGIA}

tert-butyl (2S)-2-(9H-fluoren-9-ylmethoxycarbonylamino)-3-(3',4'dimethoxyphenyl)propionate chloroform solvate

a-benzyl-N-((9H-fluoren-9-ylmethoxy)carbonyl)phenylalanine hexane solvate

$N$-((9H-Fluoren-9-ylmethoxy)carbonyl)phenylalanyl-phenylalanine hydrate

$N$-((9H-fluoren-9-ylmethoxy)carbonyl)phenylalanine

$N$-((9H-Ffluoren-9-ylmethoxy)carbonyl)phenylalanine methanol solvate

$N$-((9H-fluoren-9-ylmethoxy)carbonyl)tyrosine monohydrate

(Young, 2002) 


\section{UGIYUQ}

(Liyanage, 2016)

\section{UQOGUE}

(Liyanage, 2016)

\section{WATSIU01}

(Stefanowicz et al.,

2011)

\section{XATJAG}

(Pizzi et al., 2017)

\section{XATJEK}

(Pizzi et al., 2017)

XATKEL

(Pizzi et al., 2017)

XATKIP

(Pizzi et al., 2017)

VERXUO

(Rajbhandry et al., 2018)

\section{$N$-((9H-fluoren-9-ylmethoxy)carbonyl)-4-methylphenylalanine}

4-cyano- $N$-((9H-fluoren-9-ylmethoxy)carbonyl)phenylalanine 4-nitro- $N((9 \mathrm{H}$-fluoren-9-

ylmethoxy)carbonyl)phenylalanine

(2S)-3-(4-Amino-3-nitrophenyl)-2-(9H-fluoren-9-ylmethoxycarbonylamino)propanoic acid dichloromethane solvate

4-bromo- $N$-(((9H-fluoren-9-yl)methoxy)carbonyl)phenylalanine hydrate

4-chloro-N-(((9H-fluoren-9-yl)methoxy)carbonyl)phenylalanine

$N$-(((9H-fluoren-9-yl)methoxy)carbonyl)-4-iodophenylalanine hydrate

$N$-(((9H-fluoren-9-yl)methoxy)carbonyl)-4-fluorophenylalanine dimethyl sulfoxide solvate

$\mathrm{N}$-((9H-fluoren-9-ylmethoxy)carbonyl)phenylalanine dimethyl sulfoxide solvate

\section{Table S2}

Names of other Fmoc-AA derivatives.

\section{Fmoc-ALANINE (FmocA) derivatives}

\begin{tabular}{|c|c|}
\hline $\begin{array}{l}\text { ADAGUK } \\
\quad(\text { Xing et al., 2017) }\end{array}$ & $N$-\{[(9H-fluoren-9-yl)methoxy]carbonyl $\}-3-(2 \mathrm{H}$-isoindol-1-yl)alanine \\
\hline $\begin{array}{l}\text { CUWKIO01 } \\
\text { (Al-Mahamad et al., } \\
\text { 2017) }\end{array}$ & $N$ - $\{[(9 \mathrm{H}$-fluoren-9-yl)methoxy]carbonyl $\}$ alanine monohydrate \\
\hline $\begin{array}{l}\text { CUWKOU01 } \\
\text { (Hammarston et al., } \\
\text { 2013) }\end{array}$ & $N$-((9H-fluoren-9-ylmethoxy)carbonyl)-2-methylalanine \\
\hline
\end{tabular}

\section{Fmoc-CYSTEINE (FmocC) derivatives}

EJEWUL

$N$-Fluorenylmethoxycarbonyl-N,S-dimethyl- $L$-cysteine

(Liu et al., 2002)

\section{Fmoc-GLYCINE (FmocG) derivatives}

\begin{tabular}{|c|c|}
\hline $\begin{array}{l}\text { NOVTOJ } \\
\text { (Wu et al., 2015) }\end{array}$ & $\begin{array}{l}\mathrm{N} \text {-(Benzylsulfonyl)-N-(2-(((9H-fluoren-9-ylmethoxy)carbonyl)amino)propyl)glycine isopropano } \\
\text { solvate }\end{array}$ \\
\hline $\begin{array}{l}\text { VERQER } \\
\text { (Rajbhandary et al., } \\
\text { 2018) }\end{array}$ & $N$-((9H-fluoren-9-ylmethoxy)carbonyl)-N-(pentafluorobenzyl)glycine \\
\hline $\begin{array}{l}\text { VERQIV } \\
\text { (Rajbhandary et al., } \\
\text { 2018) }\end{array}$ & $N$-((9H-fluoren-9-ylmethoxy)carbonyl)-N-(3-fluorobenzyl)glycine \\
\hline $\begin{array}{l}\text { VERQOB } \\
\text { (Rajbhandary et al., } \\
\text { 2018) }\end{array}$ & $N$-((9H-fluoren-9-ylmethoxy)carbonyl)-N-(pentafluorobenzyl)glycine hydrate \\
\hline $\begin{array}{l}\text { VERXIC } \\
\text { (Rajbhandary et al., } \\
\text { 2018) }\end{array}$ & $N$-((9H-fluoren-9-ylmethoxy)carbonyl)-N-(4-nitrobenzyl)glycine \\
\hline $\begin{array}{l}\text { VERXOI } \\
\text { (Rajbhandary et al., }\end{array}$ & $N$-benzyl-N-((9H-fluoren-9-ylmethoxy)carbonyl)glycine \\
\hline
\end{tabular}


Fmoc-ISOLEUCINE (FmocI) derivatives

QOFHID

$N$-(Fluoren-9-ylmethoxycarbonyl)- $L$-isoleucine

(Yamada et. al, 2008)

[Synonym: Fluoren-9-ylmethyl N-(1-carboxy-2-methylbutyl)carbamate]

\section{Fmoc-LEUCINE(FmocL) derivatives}

\section{BIZXUE $N$-((Fluoren-9-yl)methoxycarbonyl)- $L$-leucine}

(Yamada et. al, 2008) [Synonym: Fluoren-9-ylmethyl N-(1-carboxy-3-methylbutyl)carbamate]

Fmoc-ORNITHINE derivatives

EXOFAY $N$-(9-Fluorenyl)methoxycarbonyl- $L$-ornithine hydrochloride diethyl ether clathrate

(Mazur et. al., 2002)

\section{Fmoc-SERINE (FmocS) derivatives}

ADAGOE $N$ - $\{[(9 \mathrm{H}-$ fluoren-9-yl)methoxy $]$ carbonyl $\}$ serine monohydrate

(Pengyao et al., 2017)

MOHCIW $N$ - $\alpha$-Fluoren-9-ylmethoxycarbonyl-O-t-butyl- $L$-serine

(Yamada et. al, 2008)

\section{Fmoc-TRYPTOPHAN (FmocW) derivatives}

\section{DIZNIK}

(Blaser et al., 2008) methyl Na-(fluoren-9-ylmethoxycarbonyl)-5-methoxy- $L$-tryptophan deuterochloroform solvate

\section{Table S3}

Crystal data of other Fmoc-AA derivatives.

\begin{tabular}{|c|c|c|c|c|c|c|c|c|c|c|}
\hline & \multirow{13}{*}{ ALA } & ADAGUK & CUWKIO01 & CUWKOU01 & \multirow{13}{*}{ GLY } & NOVTOJ & VERQIV & VERQER & VRRQOB & VERXIC \\
\hline $\begin{array}{l}\text { Struct. } \\
\text { formula }\end{array}$ & & $\mathrm{C}_{26} \mathrm{H}_{22} \mathrm{~N}_{2} \mathrm{O}_{4}$ & $\begin{array}{l}\mathrm{C}_{18} \mathrm{H}_{17} \mathrm{NO}_{4} \\
. \mathrm{H}_{2} \mathrm{O}\end{array}$ & & & $\begin{array}{l}\mathrm{C}_{27} \mathrm{H}_{28} \mathrm{~N}_{2} \\
\mathrm{O}_{6} \mathrm{~S} \\
\mathrm{C}_{3} \mathrm{H}_{8} \mathrm{O}\end{array}$ & $\mathrm{C}_{24} \mathrm{H}_{20} \mathrm{FNO}_{4}$ & $\mathrm{C}_{24} \mathrm{H}_{16} \mathrm{~F}_{5} \mathrm{NO}_{4}$ & $\begin{array}{l}\mathrm{C}_{24} \mathrm{H}_{16} \mathrm{~F}_{5} \mathrm{NO}_{4}, \\
0.1 \mathrm{H}_{2} \mathrm{O}\end{array}$ & $\mathrm{C}_{24} \mathrm{H}_{20} \mathrm{~N}_{2} \mathrm{O}_{6}$ \\
\hline Crystal syst & & monoclinic & Orthor. & $\begin{array}{l}\text { orthorhomb } \\
\text { ic }\end{array}$ & & $\begin{array}{l}\text { monoclin } \\
\text { ic }\end{array}$ & monoclinic & monoclinic & monoclinic & monoclinic \\
\hline Space gr. & & $P 2_{1}$ & $P 2_{1} 2_{1} 2_{1}$ & $P$ bcn & & $P 2_{1}$ & $P 2{ }_{1} / \mathrm{c}$ & $P 2_{1} / \mathrm{c}$ & $I 2 / \mathrm{a}$ & $P$ bca \\
\hline density & & 1.301 & 1.315 & 1.248 & & 1.311 & 1.391 & 1.518 & 1.496 & 1.404 \\
\hline $\mathrm{a}[\AA]$ & & $9.494(5)$ & $7.244(0)$ & $29.823(2)$ & & $5.038(0)$ & $12.093(3)$ & $16.793(1)$ & $21.895(11)$ & $12.115(3)$ \\
\hline $\mathrm{b}[\AA]$ & & $10.567(6)$ & $9.268(0)$ & $12.128(1)$ & & $22.705(0)$ & $9.210(1)$ & $7.741(0)$ & $6.597(3)$ & $9.187(2)$ \\
\hline c $[\AA]$ & & $10.951(6)$ & $24.780(0)$ & $9.579(0)$ & & $12.728(0)$ & $35.336(8)$ & $16.195(0)$ & $29.900(20)$ & $36.754(9)$ \\
\hline$\alpha\left[{ }^{0}\right]$ & & 90.00 & 90.00 & 90.00 & & 90.00 & 90.00 & 90.00 & 90.00 & 90.00 \\
\hline$\beta\left[^{0}\right]$ & & $97.68(0)$ & 90.00 & 90.00 & & $98.45(0)$ & $100.31(0)$ & $97.05(0)$ & $99.93(0)$ & 90.00 \\
\hline$\gamma\left[{ }^{\circ}\right]$ & & 90.00 & 90.00 & 90.00 & & 90.00 & 90.00 & 90.00 & 90.00 & 90.00 \\
\hline $\mathrm{R} 1[\mathrm{I}>2 \sigma(\mathrm{I})]$ & & 6.35 & 2.49 & 4.06 & & 5.94 & 8.80 & 5.11 & 8.29 & 5.17 \\
\hline \multirow[t]{2}{*}{ Temp. } & & 298 & 150 & 298 & & 100 & 100 & 100 & 100 & 100 \\
\hline & VERXOI & XAVYIE & \multirow{13}{*}{ CYST. } & EJEWUL & \multirow{13}{*}{ ILE } & QOFHID & \multirow{13}{*}{ LEU } & BIZXUE & \multirow{13}{*}{ ORN } & EXOFAY \\
\hline $\begin{array}{l}\text { Structural } \\
\text { formula }\end{array}$ & $\mathrm{C}_{24} \mathrm{H}_{21} \mathrm{NO}_{4}$ & $\begin{array}{l}\mathrm{C}_{28} \mathrm{H}_{36} \mathrm{~N}_{2} \mathrm{O}_{6}, \\
\mathrm{CH}_{4} \mathrm{O}\end{array}$ & & $\begin{array}{l}\mathrm{C}_{20} \mathrm{H}_{21} \mathrm{NO}_{4} \\
\mathrm{~S}\end{array}$ & & $\begin{array}{l}\mathrm{C}_{21} \mathrm{H}_{23} \mathrm{~N} \\
\mathrm{O}_{4}\end{array}$ & & $\mathrm{C} 21 \mathrm{H} 23 \mathrm{NO} 4$ & & $\begin{array}{l}\mathrm{C}_{20} \mathrm{H}_{23} \mathrm{~N}_{2} \mathrm{O}_{4}, \mathrm{Cl}, \\
\mathrm{C}_{4} \mathrm{H}_{10} \mathrm{O}\end{array}$ \\
\hline Crystal syst & monoclinic & monoclinic & & monoclinic & & Orthorh. & & Orthorh. & & monoclinic \\
\hline Space gr. & $P 2_{1} / \mathrm{c}$ & $P 2_{1} / \mathrm{n}$ & & $\mathrm{P} 21$ & & $P 2_{1} 2_{1} 2_{1}$ & & $P 2_{1} 2_{1} 2_{1}$ & & $\mathrm{P} 21$ \\
\hline density & 1.319 & 1.269 & & 1.281 & & 1.273 & & 1.228 & & 1.265 \\
\hline $\mathrm{a}[\AA]$ & $9.327(2)$ & $8.532(1)$ & & $11.262(1)$ & & $5.334(0)$ & & $5.495(0)$ & & $5.094(1)$ \\
\hline $\mathrm{b}[\AA]$ & $34.646(9)$ & $9.475(1)$ & & $6.448(0)$ & & $13.697(0)$ & & $14.270(0)$ & & $15.499(3)$ \\
\hline $\mathrm{c}[\AA \AA]$ & $12.074(3)$ & $34.448(3)$ & & 13.281(1) & & $25.251(0)$ & & $24.376(0)$ & & $15.575(3)$ \\
\hline$\alpha\left[{ }^{\circ}\right]$ & 90.00 & 90.00 & & 90.00 & & 90.00 & & 90.00 & & 90.00 \\
\hline$\beta\left[{ }^{\circ}\right]$ & $91.12(0)$ & 96.31(1) & & $92.66(0)$ & & 90.00 & & 90.00 & & $96.74(3)$ \\
\hline$\gamma\left[{ }^{\circ}\right]$ & 90.00 & 90.00 & & 90.00 & & 90.00 & & 90.00 & & 90.00 \\
\hline $\mathrm{R} 1[\mathrm{I}>2 \sigma(\mathrm{I})]$ & 7.29 & 5.06 & & 3.65 & & 3.40 & & 3.93 & & 5.43 \\
\hline \multirow[t]{2}{*}{ Temp. } & 100 & 123 & & 293 & & 90 & & 150 & & 293 \\
\hline & \multirow[t]{3}{*}{ SER } & ADAGOE & MOHCIW & \multirow[t]{3}{*}{ TRP } & DIZNIK & & & & & \\
\hline $\begin{array}{l}\text { Structural } \\
\text { formula }\end{array}$ & & $\begin{array}{l}\mathrm{C}_{18} \mathrm{H}_{17} \mathrm{NO}_{5}, \\
\mathrm{H}_{2} \mathrm{O}\end{array}$ & $\mathrm{C}_{22} \mathrm{H}_{25} \mathrm{NO}_{6}$ & & $\begin{array}{l}\mathrm{C}_{28} \mathrm{H}_{26} \mathrm{~N}_{2} \mathrm{O} \\
{ }_{5} \cdot \mathrm{CDCl}_{3}\end{array}$ & & & & & \\
\hline Crystal syst & & orthorhombic & monoclinic & & monoclinic & & & & & \\
\hline
\end{tabular}




\begin{tabular}{|c|c|c|c|}
\hline Space gr. & $P 2_{1} 2_{1} 2_{1}$ & $P 2_{1}$ & $P 2_{1}$ \\
\hline density & 1.374 & 1.221 & 1.402 \\
\hline $\mathrm{a}[\AA]$ & $7.388(10)$ & $5.844(0)$ & $11.439(1)$ \\
\hline $\mathrm{b}[\AA]$ & $9.215(13)$ & $11.937(0)$ & $5.867(0)$ \\
\hline c $[\AA]$ & $24.530(30)$ & $15.042(1)$ & $21.531(2)$ \\
\hline$\alpha\left[^{0}\right]$ & 90.00 & 90.00 & 90.00 \\
\hline$\beta\left[^{0}\right]$ & 90.00 & $96.20(0)$ & 104.44(1) \\
\hline$\gamma\left[^{\circ}\right]$ & 90.00 & 90.00 & 90.00 \\
\hline $\mathrm{R} 1[\mathrm{I}>2 \sigma(\mathrm{I})]$ & 4.75 & 5.36 & 3.35 \\
\hline Temp. & 298 & 290 & 120 \\
\hline
\end{tabular}

\section{Table S4}

Hydrogen-bond geometry $\left(\AA,^{\circ}\right)$ for Fmoc-Tyr/Phe derivatives.

\begin{tabular}{|c|c|c|c|c|c|}
\hline & $D$-H & $\mathrm{H}^{\cdots} A$ & $D^{\cdots} A$ & $D-\mathrm{H}^{\cdots} A$ & Symmetry codes \\
\hline \multicolumn{6}{|c|}{ group $\boldsymbol{A}$} \\
\hline \multicolumn{6}{|l|}{ CAMLEK } \\
\hline $\mathrm{O} 3-\mathrm{H} 1 \cdots \mathrm{O} 4$ & 0.89 & 1.82 & $2.681(4)$ & $162(5)$ & $1-x,-1 / 2+y, 1-z$ \\
\hline $\mathrm{O} 5-\mathrm{H} 2 \cdots \mathrm{O} 2$ & 0.87 & 1.92 & $2.754(4)$ & $162(5)$ & $2-x, 1 / 2+y, 1-z$ \\
\hline *O6-H3 $\cdots$ O5 & 0.84 & 2.25 & $2.680(5)$ & 112 & \\
\hline $\mathrm{N} 1-\mathrm{H} 4 \cdots \mathrm{O} 3$ & 0.94 & 2.45 & $3.386(5)$ & $172(4)$ & $x, 1+y, z$ \\
\hline *C14-H14 $\cdots \mathrm{O} 2$ & 0.99 & 2.41 & $2.761(6)$ & 100 & \\
\hline $\mathrm{C} 14-\mathrm{H} 15 \cdots \mathrm{O} 2$ & 0.99 & 2.34 & $3.284(7)$ & 159 & $x, 1+y, z$ \\
\hline $\mathrm{C} 16-\mathrm{H} 16 \cdots \mathrm{O} 1$ & 1.00 & 2.36 & $3.282(6)$ & 154 & $x,-1+y, z$ \\
\hline${ }^{*} \mathrm{C} 18-\mathrm{H} 17 \cdots \mathrm{O} 3$ & 0.99 & 2.59 & $2.935(5)$ & 100 & \\
\hline \multicolumn{6}{|l|}{ OGOGIA } \\
\hline $\mathrm{N} 1-\mathrm{H} 1 \cdots \mathrm{O} 2$ & 0.88 & 2.20 & $3.075(5)$ & 171 & $-1+x, y, z$ \\
\hline${ }^{*} \mathrm{C} 14-\mathrm{H} 12 \cdots \mathrm{O} 2$ & 0.99 & 2.30 & $2.741(5)$ & 106 & \\
\hline$* \mathrm{C} 16-\mathrm{H} 13 \cdots \mathrm{O} 2$ & 1.00 & 2.45 & $2.795(5)$ & 100 & \\
\hline $\mathrm{C} 18-\mathrm{H} 15 \cdots \mathrm{N} 4$ & 0.98 & 2.60 & $3.559(10)$ & 167 & $5 / 2-x, 1-y, 1 / 2+z$ \\
\hline $\mathrm{C} 26-\mathrm{H} 24 \cdots \mathrm{O} 3$ & 0.99 & 2.44 & $3.352(6)$ & 153 & $1-x, 1 / 2+y, 1 / 2-z$ \\
\hline \multicolumn{6}{|l|}{ OGIYAG } \\
\hline $\mathrm{O} 4-\mathrm{H} 1 \cdots \mathrm{O} 6$ & 0.98 & 1.60 & $2.577(3)$ & $174(4)$ & $1 / 2+x,-1 / 2+y, z$ \\
\hline $\mathrm{O} 5-\mathrm{H} 2 \cdots \mathrm{O} 3$ & 0.91 & 1.86 & $2.708(3)$ & $155(3)$ & $1-x, y,-z$ \\
\hline$* \mathrm{~N} 1-\mathrm{H} 3 * \mathrm{O} 3$ & 0.91 & 2.31 & $2.682(3)$ & 104(3) & \\
\hline O6-H22 $\cdots 5$ & 0.91 & 1.85 & $2.759(4)$ & $174(4)$ & $1 / 2-x,-1 / 2+y,-z$ \\
\hline $\mathrm{O} 6-\mathrm{H} 23 \cdots \mathrm{O} 2$ & & & & & $0.95(4)$ \\
\hline $\mathrm{C} 14-\mathrm{H} 14 \cdots \mathrm{O} 2$ & 1.04 & 2.39 & $3.565(5)$ & $155(2)$ & $x, 1+y, z$ \\
\hline $\mathrm{C} 16-\mathrm{H} 15 \cdots \mathrm{O} 1$ & 1.02 & 2.47 & $3.237(4)$ & $131.4(19)$ & $x,-1+y, z$ \\
\hline${ }^{*} \mathrm{C} 18-\mathrm{H} 16 \cdots \mathrm{O} 2$ & 0.97 & 2.56 & $3.187(4)$ & $122.4(19)$ & \\
\hline $\mathrm{C} 18-\mathrm{H} 17 \cdots \mathrm{O} 1$ & 1.00 & 2.58 & $3.172(4)$ & $117.8(19)$ & $x,-1+y, z$ \\
\hline \multicolumn{6}{|l|}{ INEJEQ } \\
\hline $\mathrm{N} 1-\mathrm{H} 22 \cdots \mathrm{O} 5$ & 0.88 & 2.36 & $3.205(3)$ & 161 & $x, 1+y, z$ \\
\hline $\mathrm{C} 1-\mathrm{H} 1 \cdots \mathrm{O} 5$ & 0.95 & 2.42 & $3.305(4)$ & 154 & $x, 1+y, z$ \\
\hline $\mathrm{C} 13-\mathrm{H} 13 \cdots \mathrm{O} 3$ & 0.98 & 2.59 & $3.488(4)$ & 153 & $x,-1+y, z$ \\
\hline${ }^{*} \mathrm{C} 13-\mathrm{H} 14 \cdots \mathrm{O} 3$ & 0.98 & 2.45 & $2.997(4)$ & 115 & \\
\hline${ }^{*} \mathrm{C} 14-\mathrm{H} 17 \cdots \mathrm{O} 3$ & 0.98 & 2.54 & $3.056(4)$ & 113 & \\
\hline \multicolumn{6}{|c|}{ group $\boldsymbol{B}$} \\
\hline \multicolumn{6}{|l|}{ OGIXOT } \\
\hline $\mathrm{O} 3-\mathrm{H} 1 \cdots \mathrm{O} 4^{\mathrm{i}}$ & 0.84 & 1.80 & $2.633(4)$ & 173 & $-x,-1 / 2+y, 1-z$ \\
\hline $\mathrm{N} 1-\mathrm{H} 8 \cdots \mathrm{O} 2^{\mathrm{ii}}$ & 0.84 & 2.08 & $2.904(4)$ & $168(3)$ & $x,-1+y, z$ \\
\hline${ }^{*} \mathrm{C} 16-\mathrm{H} 5 \cdots \mathrm{O} 2$ & 0.97 & 2.37 & $2.807(5)$ & $106(3)$ & \\
\hline \multicolumn{6}{|l|}{ VERXUO } \\
\hline $\mathrm{O} 4-\mathrm{H} 1 \cdots \mathrm{S} 1$ & 0.84 & 2.80 & $3.576(10)$ & 154 & \\
\hline $\mathrm{O} 4-\mathrm{H} 1 \cdots \mathrm{O} 9$ & 0.84 & 1.73 & $2.564(13)$ & 172 & \\
\hline $\mathrm{N} 1-\mathrm{H} 2 \cdots \mathrm{O} 2$ & 0.88 & 2.10 & $2.922(14)$ & 156 & $1+x, y, z$ \\
\hline $\mathrm{O} 8-\mathrm{H} 22 \cdots \mathrm{O} 10$ & 0.84 & 1.74 & $2.555(16)$ & 164 & $1+x, y, z$ \\
\hline N2-H23 ․ O6 & 0.88 & 2.08 & $2.933(14)$ & 161 & \\
\hline C5-H6 $\cdots 9$ & 0.95 & 2.58 & $3.437(19)$ & 150 & $x, 1+y, z$ \\
\hline *C16-H14 $\cdots$ O9 & 1.00 & 2.41 & $2.811(17)$ & 103 & \\
\hline${ }^{*} \mathrm{C} 18-\mathrm{H} 16 \cdots \mathrm{O} 4$ & 0.99 & 2.50 & $2.859(15)$ & 101 & \\
\hline${ }^{*} \mathrm{C} 40-\mathrm{H} 35 \cdots \mathrm{O} 6$ & 1.00 & 2.40 & $2.827(17)$ & 105 & \\
\hline $\mathrm{C} 49-\mathrm{H} 43 \cdots \mathrm{O} 3$ & 0.98 & 2.46 & $3.296(19)$ & 143 & \\
\hline $\mathrm{C} 49-\mathrm{H} 44 \cdots \mathrm{O} 7$ & 0.98 & 2.49 & $3.208(19)$ & 130 & $-1+x,-1+y, 1+z$ \\
\hline $\mathrm{C} 50-\mathrm{H} 47 \cdots \mathrm{O} 9$ & 0.98 & 2.56 & $3.394(19)$ & 142 & $-1+x, y, z$ \\
\hline $\mathrm{C} 51-\mathrm{H} 49 \cdots \mathrm{O} 3$ & 0.93 & 2.26 & $3.14(2)$ & 157 & $x, 1+y,-1+z$ \\
\hline $\mathrm{C} 52-\mathrm{H} 53 \cdots \mathrm{O} 10$ & 0.98 & 2.13 & $3.00(3)$ & 147 & $1+x, y, z$ \\
\hline $\mathrm{C} 52-\mathrm{H} 58 \cdots \mathrm{O} 10$ & 1.10 & 2.55 & $3.00(3)$ & 103 & $1+x, y, z$ \\
\hline \multicolumn{6}{|l|}{ OGIXUZ } \\
\hline $\mathrm{O} 3-\mathrm{H} 1 \cdots \mathrm{O} 5$ & 0.84 & 1.71 & $2.539(19)$ & 170 & $1-x,-1 / 2+y, 1-z$ \\
\hline $\mathrm{N} 1-\mathrm{H} 2 \cdots \mathrm{O} 2$ & 0.88 & 2.05 & $2.857(18)$ & 152 & $x,-1+y, z$ \\
\hline O5-H22 $\cdots 6$ & 0.84 & 1.94 & $2.712(19)$ & 152 & \\
\hline O6-H26 $\cdots 4$ & 0.84 & 1.98 & $2.788(19)$ & 162 & $x, 1+y, z$ \\
\hline C4-H5 $\cdots 06$ & 0.95 & 2.59 & $3.49(2)$ & 156 & $2-x,-1 / 2+y, 1-z$ \\
\hline$* \mathrm{C} 16-\mathrm{H} 14 \cdots \mathrm{O} 2$ & 1.00 & 2.43 & $2.83(2)$ & 103 & \\
\hline${ }^{*} \mathrm{C} 18-\mathrm{H} 16 \cdots \mathrm{O} 3$ & 0.99 & 2.55 & $2.91(2)$ & 101 & \\
\hline
\end{tabular}




\begin{tabular}{|c|c|c|c|c|c|}
\hline $\mathrm{C} 25-\mathrm{H} 23 \cdots \mathrm{O} 3$ & 0.98 & 2.60 & $3.52(3)$ & 157 & $1-x,-1 / 2+y, 1-z$ \\
\hline $\mathrm{C} 25-\mathrm{H} 24 \cdots \mathrm{O} 6$ & 0.98 & 2.55 & $3.48(3)$ & 157 & $x,-1+y, z$ \\
\hline \multicolumn{6}{|l|}{ EKEWUM } \\
\hline $\mathrm{N} 2-\mathrm{H} 11 \cdots \mathrm{O} 2$ & 0.88 & 2.47 & $3.213(5)$ & 142 & \multirow[t]{4}{*}{$1+x, y, z$} \\
\hline 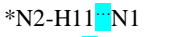 & 0.88 & 2.59 & $3.042(7)$ & 113 & \\
\hline$* \mathrm{C} 6-\mathrm{H} 5 \cdots \mathrm{O} 2$ & 0.99 & 2.46 & $2.849(6)$ & 103 & \\
\hline${ }^{*} \mathrm{C} 7-\mathrm{H} 7 \cdots \mathrm{O} 3$ & 1.00 & 2.37 & $2.810(7)$ & 106 & \\
\hline $\mathrm{C} 7-\mathrm{H}^{2} \cdots \mathrm{O} 4$ & 1.00 & 2.59 & $3.396(6)$ & 137 & \multirow[t]{2}{*}{$-1+x, y, z$} \\
\hline$*^{\mathrm{C}} 11-\mathrm{H} 12 \cdots \mathrm{O} 3$ & 0.99 & 2.24 & $2.688(6)$ & 106 & \\
\hline $\mathrm{C} 11-\mathrm{H} 13 \cdots \mathrm{O} 3$ & 0.99 & 2.45 & $3.188(6)$ & 131 & $1+x, y, z$ \\
\hline $\mathrm{C} 24-\mathrm{H} 22 \cdots \mathrm{O} 3$ & 0.95 & 2.54 & $3.355(6)$ & 144 & $1+x, y, z$ \\
\hline \multicolumn{6}{|l|}{ NUBPEH } \\
\hline $\mathrm{N} 1-\mathrm{H} 2 \cdots \mathrm{O} 3$ & 0.88 & 2.01 & $2.779(18)$ & 146 & \multirow{4}{*}{$\begin{array}{l}x, 1+y, z \\
x,-1+y, z\end{array}$} \\
\hline $\mathrm{N} 2-\mathrm{H} 3 \cdots \mathrm{O} 4$ & 0.88 & 2.04 & $2.908(15)$ & 170 & \\
\hline${ }^{*} \mathrm{C} 8-\mathrm{H} 11 \cdots \mathrm{O} 4$ & 1.00 & 2.36 & $2.767(19)$ & 103 & \\
\hline${ }^{*} \mathrm{C} 10-\mathrm{H} 12 \cdots \mathrm{O} 3$ & 1.00 & 2.43 & $2.87(2)$ & 105 & \\
\hline \multirow[t]{2}{*}{$\mathrm{C} 27-\mathrm{H} 24 \cdots \mathrm{O} 3$} & 0.99 & 2.56 & $3.289(17)$ & 131 & $x, 1+y, z$ \\
\hline & \multicolumn{5}{|c|}{ group $C$} \\
\hline MOXSUP & & & & & \\
\hline $\mathrm{O} 3-\mathrm{H} 1 \cdots \mathrm{O} 4$ & 0.92 & 1.77 & $2.684(3)$ & $176(3)$ & $1-x, 1-y, 1-z$ \\
\hline *N1-H2 $\cdots 4$ & 0.82 & 2.17 & $2.624(3)$ & $115(3)$ & \\
\hline $\mathrm{C} 2-\mathrm{H} 3 \cdots \mathrm{O} 2$ & 0.99 & 2.56 & $3.273(4)$ & 129 & $-1+x, y, z$ \\
\hline *C18-H14 & 0.99 & 2.46 & $3.104(4)$ & 122 & \\
\hline $\mathrm{C} 25-\mathrm{H} 21 \cdots \mathrm{O} 4$ & 0.99 & 2.54 & $3.502(4)$ & 163 & $1+x, y, z$ \\
\hline$* \mathrm{C} 25-\mathrm{H} 22 \cdots \mathrm{O} 2$ & 0.99 & 2.57 & $3.189(4)$ & 121 & \\
\hline DULLAZ & & & & & \\
\hline $\mathrm{O} 2-\mathrm{H} 1 \cdots \mathrm{O} 5$ & 0.84 & 1.75 & $2.592(16)$ & 180 & \\
\hline$* \mathrm{~N} 1-\mathrm{H} 2 \cdots \mathrm{O} 1$ & 0.88 & 2.19 & $2.616(16)$ & 109 & \\
\hline O6-H30 $\cdots 1$ & 0.84 & 1.82 & $2.661(16)$ & 179 & \\
\hline *N2-H31 $\cdots$ & 0.88 & 2.22 & $2.671(16)$ & 112 & \\
\hline${ }^{*} \mathrm{C} 4-\mathrm{H} 3 * \mathrm{O} 4$ & 0.99 & 2.40 & $3.062(19)$ & 124 & \\
\hline $\mathrm{C} 11-\mathrm{H} 10 \cdots \mathrm{O} 3$ & 0.99 & 2.46 & $3.411(19)$ & 161 & $-1+x, y, z$ \\
\hline $\mathrm{C} 11-\mathrm{H} 11 \cdots \mathrm{O} 1$ & 0.99 & 2.59 & $3.473(19)$ & 149 & $-1+x, y, z$ \\
\hline${ }^{*} \mathrm{C} 11-\mathrm{H} 11 \cdots \mathrm{O} 2$ & 0.99 & 2.51 & $2.865(19)$ & 101 & \\
\hline$*^{\mathrm{C}} 15-\mathrm{H} 20 \cdots \mathrm{O} 4$ & 0.99 & 2.35 & $2.78(2)$ & 105 & \\
\hline *C $32-\mathrm{H} 32 \cdots \mathrm{O} 8$ & 0.99 & 2.56 & $3.161(19)$ & 119 & \\
\hline${ }^{*} \mathrm{C} 39-\mathrm{H} 39 \cdots \mathrm{O} 6$ & 0.99 & 2.54 & $2.875(19)$ & 100 & \\
\hline${ }^{*} \mathrm{C} 39-\mathrm{H} 40 \cdots \mathrm{O} 8$ & 0.99 & 2.51 & $3.062(19)$ & 115 & \\
\hline 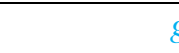 & & & & & \\
\hline WATSIU01 & & & & & \\
\hline $\mathrm{O} 2-\mathrm{H} 1 \cdots \mathrm{O} 5$ & 0.84 & 1.80 & $2.631(3)$ & 173 & $1-x, 1 / 2+y,-z$ \\
\hline $\mathrm{N} 1-\mathrm{H} 2 \cdots \mathrm{O} 9$ & 0.88 & 2.35 & $3.134(3)$ & 148 & \\
\hline$* \mathrm{~N} 3-\mathrm{H} 3 \cdots \mathrm{O} 3$ & 0.88 & 2.03 & $2.643(3)$ & 126 & \\
\hline $\mathrm{N} 3-\mathrm{H} 3 \cdots \mathrm{O} 10$ & 0.88 & 2.32 & $2.958(3)$ & 129 & $x,-1+y, z$ \\
\hline $\mathrm{N} 3-\mathrm{H} 4 \cdots \mathrm{O} 1$ & 0.88 & 2.08 & $2.932(3)$ & 163 & $x,-1+y, z$ \\
\hline $\mathrm{O} 8-\mathrm{H} 22 \cdots \mathrm{O} 11$ & 0.84 & 1.83 & $2.669(3)$ & 177 & $1-x, 1 / 2+y, 1-z$ \\
\hline N4-H23 -O3 & 0.88 & 2.37 & $3.186(3)$ & 154 & $x, 1+y, z$ \\
\hline $\mathrm{N} 6-\mathrm{H} 24 \cdots \mathrm{O} 7$ & 0.88 & 2.13 & 2.977(3) & 161 & $x,-1+y, z$ \\
\hline N6-H25 & 0.88 & 2.39 & $2.942(3)$ & 121 & \\
\hline *N6-H25 *O & 0.88 & 2.01 & $2.630(3)$ & 126 & \\
\hline $\mathrm{Cl} 4-\mathrm{H} 48 \cdots \mathrm{Cl} 10$ & 1.73 & 2.81 & $3.999(3)$ & 122 & $1+x, y, z$ \\
\hline$* \mathrm{C} 2-\mathrm{H} 5 \cdots \mathrm{O} 5$ & 1.00 & 2.33 & $2.778(3)$ & 106 & \\
\hline $\mathrm{C} 8-\mathrm{H} 9 \cdots \mathrm{O} 5$ & 0.95 & 2.59 & $3.494(3)$ & 160 & $1-x,-1 / 2+y,-z$ \\
\hline${ }^{*} \mathrm{C} 26-\mathrm{H} 26 \cdots \mathrm{O} 11$ & 1.00 & 2.42 & $2.831(3)$ & 104 & \\
\hline $\mathrm{C} 29-\mathrm{H} 29 \cdots \mathrm{O} 3$ & 0.95 & 2.50 & $3.277(3)$ & 139 & $x, 1+y, z$ \\
\hline $\mathrm{C} 32-\mathrm{H} 30 \cdots \mathrm{O} 11$ & 0.95 & 2.41 & $3.326(3)$ & 162 & $1-x,-1 / 2+y, 1-z$ \\
\hline $\mathrm{C} 49-\mathrm{H} 43 \cdots \mathrm{O} 1$ & 0.99 & 2.49 & $3.170(4)$ & 125 & $-1+x, y, z$ \\
\hline $\mathrm{C} 50-\mathrm{H} 45 \cdots \mathrm{O} 4$ & 0.99 & 2.35 & $3.239(6)$ & 149 & $1-x, 1 / 2+y, 1-z$ \\
\hline $\mathrm{C} 50-\mathrm{H} 47 \cdots \mathrm{O} 7$ & 0.79 & 2.50 & $3.165(6)$ & 143 & $1-x,-1 / 2+y, 1-z$ \\
\hline UQOGUE & & & & & \\
\hline 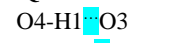 & 0.84 & 1.81 & $2.644(6)$ & 169 & $-1 / 2+x, 1 / 2-y,-z$ \\
\hline $\mathrm{C} 10-\mathrm{H} 9 \cdots \mathrm{O} 6$ & 0.95 & 2.47 & $3.137(18)$ & 127 & $1+x,-1+y, z$ \\
\hline $\mathrm{C} 11-\mathrm{H} 10 \cdots \mathrm{N} 2$ & 0.95 & 2.59 & $3.312(13)$ & 133 & $x,-1+y, z$ \\
\hline $\mathrm{C} 14-\mathrm{H} 13 \cdots \mathrm{O} 2$ & 0.99 & 2.30 & $3.188(8)$ & 148 & $1+x, y, z$ \\
\hline $\mathrm{C} 16-\mathrm{H} 14 \cdots \mathrm{O} 1$ & 1.00 & 2.33 & $3.185(7)$ & 142 & $-1+x, y, z$ \\
\hline${ }^{*} \mathrm{C} 16-\mathrm{H} 14 \cdots \mathrm{O} 2$ & 1.00 & 2.42 & $2.789(7)$ & 101 & \\
\hline $\mathrm{C} 18-\mathrm{H} 16 \cdots \mathrm{N} 2$ & 0.99 & 2.35 & $3.335(13)$ & 175 & $-x,-1 / 2+y, 1 / 2-z$ \\
\hline UQIYUQ & & & & & \\
\hline $\mathrm{O} 4-\mathrm{H} 1 \cdots \mathrm{O} 3$ & 0.82 & 1.84 & $2.652(2)$ & $172(3)$ & $-1 / 2+x, 1 / 2-y,-z$ \\
\hline $\mathrm{N} 1-\mathrm{H} 2 \cdots \mathrm{O} 4$ & 0.87 & 2.59 & $3.415(2)$ & $159(2)$ & $1+x, y, z$ \\
\hline $\mathrm{C} 14-\mathrm{H} 13 \cdots \mathrm{O} 2$ & 0.97 & 2.27 & $3.138(3)$ & $149(2)$ & $1+x, y, z$ \\
\hline $\mathrm{C} 16-\mathrm{H} 14 \cdots \mathrm{O} 1$ & 0.96 & 2.38 & $3.222(2)$ & $147(2)$ & $-1+x, y, z$ \\
\hline${ }^{*} \mathrm{C} 16-\mathrm{H} 14 \cdots \mathrm{O} 2$ & $0.96(2)$ & $2.35(3)$ & $2.790(2)$ & $107(2)$ & \\
\hline & & & & & \\
\hline XATKEL & & & & & \\
\hline N1-H13 “-O2 & 0.88 & 2.09 & $2.90(4)$ & 154 & $x, y, l+z$ \\
\hline $\mathrm{O} 3-\mathrm{H} 20 \cdots \mathrm{O} 4$ & 0.84 & 1.75 & $2.58(4)$ & 170 & $y,-x+y,-1 / 2+z$ \\
\hline $\mathrm{C} 21-\mathrm{H} 16 \cdots \mathrm{O} 4$ & 0.99 & 2.54 & $3.23(4)$ & 127 & \\
\hline$* \mathrm{C} 24-\mathrm{H} 19 * \mathrm{O} 2$ & 1.00 & 2.31 & $2.68(3)$ & 101 & \\
\hline
\end{tabular}




\begin{tabular}{|c|c|c|c|c|c|}
\hline \multicolumn{6}{|l|}{ ХАТJAG } \\
\hline $\mathrm{N} 1-\mathrm{H} 13 \cdots \mathrm{O} 2$ & 0.88 & 2.05 & $2.87(2)$ & 155 & $x, y, 1+z$ \\
\hline $\mathrm{O} 3-\mathrm{H} 20 \cdots \mathrm{O} 4$ & 0.84 & 1.76 & $2.58(3)$ & 166 & $y, 1-x+y,-1 / 2+z$ \\
\hline $\mathrm{C} 21-\mathrm{H} 16 \cdots \mathrm{O} 4$ & 0.99 & 2.54 & $3.25(2)$ & 129 & $y, 1-x+y,-1 / 2+z$ \\
\hline${ }^{*} \mathrm{C} 24-\mathrm{H} 19 \cdots \mathrm{O} 2$ & 1.00 & 2.37 & $2.732(15)$ & 100 & \\
\hline \multicolumn{6}{|l|}{ ХАТJЕК } \\
\hline $\mathrm{O} 2-\mathrm{H} 1 \cdots \mathrm{O} 4$ & 0.84 & 1.81 & $2.646(11)$ & 172 & $2-x, 1 / 2+y, 2-z$ \\
\hline $\mathrm{N} 1-\mathrm{H} 2 \cdots \mathrm{O} 3$ & 0.88 & 2.00 & $2.824(9)$ & 156 & $x, 1+y, z$ \\
\hline${ }^{*} \mathrm{C} 13-\mathrm{H} 10 \cdots \mathrm{O} 3$ & 1.00 & 2.37 & $2.806(11)$ & 105 & \\
\hline \multicolumn{6}{|l|}{ XATKIP } \\
\hline $\mathrm{N} 1-\mathrm{H} 12 \cdots \mathrm{O} 2$ & 0.88 & 2.10 & $2.849(14)$ & 142 & $-1+x, y, z$ \\
\hline $\mathrm{O} 3-\mathrm{H} 14 \cdots \mathrm{O} 5$ & 0.84 & 1.80 & $2.61(2)$ & 161 & \\
\hline $\mathrm{S} 1-\mathrm{H} 28 \cdots \mathrm{O} 5$ & 1.64 & 2.33 & $3.442(16)$ & 119 & $-1+x, y, z$ \\
\hline${ }^{*} \mathrm{C} 16-\mathrm{H} 13 \cdots \mathrm{O} 2$ & 1.00 & 2.40 & $2.820(17)$ & 105 & \\
\hline *C18-H16 $\cdots 3$ & 0.99 & 2.57 & $2.92(2)$ & 100 & \\
\hline $\mathrm{C} 25-\mathrm{H} 21 \cdots \mathrm{O} 4$ & 0.98 & 2.27 & $3.02(3)$ & 132 & $-1-x,-1 / 2+y, 1 / 2-z$ \\
\hline $\mathrm{C} 26-\mathrm{H} 24 \cdots \mathrm{O} 5$ & 0.98 & 2.26 & $3.09(3)$ & 141 & $-1+x, y, z$ \\
\hline $\mathrm{C} 25-\mathrm{H} 30 \cdots \mathrm{O} 4$ & 0.98 & 2.17 & $3.02(3)$ & 144 & $-1-x,-1 / 2+y, 1 / 2-z$ \\
\hline *intramolecular & & & & & \\
\hline
\end{tabular}

\section{Table S5}

Geometrical parameters (in $\AA$ and angles in ${ }^{\circ}$ ) for the $\pi$-stacking moieties involved in the $\pi \cdots \pi$ interactions for $F m o c$-AAs. Intercontact distances below $5 \AA$ are considered.

\begin{tabular}{|c|c|c|c|c|c|c|c|}
\hline $\mathrm{Cg}(\mathrm{I})-\mathrm{Cg}(\mathrm{J})^{\mathrm{a}, \mathrm{b}}$ & symmetry & $\mathrm{Cg} \cdots \mathrm{Cg}^{\mathrm{c}}$ & Cg(I)-perp ${ }^{d}$ & $\mathrm{Cg}(\mathrm{J})-$ perp $^{\mathrm{e}}$ & $\alpha^{f}$ & $\beta^{g}$ & $\gamma^{\mathrm{h}}$ \\
\hline \multicolumn{8}{|c|}{ group $A$} \\
\hline \multicolumn{8}{|l|}{ CAMLEK } \\
\hline $\mathrm{Cg}(1) \cdots \operatorname{Cg}(2)$ & $1-x,-1 / 2+y,-z$ & $4.760(3)$ & $-2.466(2)$ & $-4.6656(19)$ & $61.3(2)$ & 11.4 & 58.8 \\
\hline $\operatorname{Cg}(2) \cdot \operatorname{Cg}(3)$ & $1-x,-1 / 2+y,-z$ & $4.875(3)$ & $-1.7119(19)$ & $-4.6761(18)$ & $61.2(2)$ & 16.4 & 69.4 \\
\hline $\operatorname{Cg}(2) \cdot \operatorname{Cg}(4)$ & $2-x, 1 / 2+y, 1-z$ & $4.840(3)$ & $3.5746(19)$ & $3.488(2)$ & $16.3(2)$ & 43.9 & 42.4 \\
\hline $\operatorname{Cg}(3) \cdots \operatorname{Cg}(2)$ & $1-x,-1 / 2+y,-z$ & $4.778(3)$ & $-1.8397(18)$ & $-4.6560(19)$ & $61.2(2)$ & 13.0 & 67.4 \\
\hline \multicolumn{8}{|l|}{ OGOGIA } \\
\hline $\mathrm{Cg}(1) \operatorname{Cg}(2)$ & $-1+x, y, z$ & $4.956(3)$ & $3.4172(17)$ & $-3.544(2)$ & $2.6(2)$ & 44.4 & 46.4 \\
\hline \multicolumn{8}{|l|}{ OGIYAG } \\
\hline $\operatorname{Cg}(1) \cdots \operatorname{Cg}(2)$ & $1 / 2-x,-1 / 2+y, 1-z$ & $4.712(4)$ & $2.3423(9)$ & $4.5379(9)$ & $60.18(11)$ & 15.6 & 60.2 \\
\hline $\operatorname{Cg}(3) \cdot \operatorname{Cg}(2)$ & $1 / 2-x,-1 / 2+y, 1-z$ & $4.630(4)$ & $1.9203(9)$ & $4.5296(9)$ & $59.55(11)$ & 12.0 & 65.5 \\
\hline \multicolumn{8}{|l|}{ INEJEQ } \\
\hline $\operatorname{Cg}(1) \cdots \operatorname{Cg}(3)$ & $x,-1+y, z$ & $4.482(2)$ & $-3.5501(15)$ & $3.5545(14)$ & $1.47(18)$ & 37.5 & 37.6 \\
\hline $\operatorname{Cg}(1) \cdots \operatorname{Cg}(4)$ & $x, 1+y, z$ & $3.977(2)$ & $3.4739(15)$ & $-3.5410(15)$ & 2.21(19) & 27.1 & 29.1 \\
\hline $\operatorname{Cg}(2) \cdot \operatorname{Cg}(2)$ & $1-x, 1 / 2+y, 1-z$ & $4.8741(18)$ & $1.0324(12)$ & $4.6644(12)$ & $84.97(14)$ & 16.9 & 77.8 \\
\hline $\mathrm{Cg}(3) \cdots \operatorname{Cg}(1)$ & $x, 1+y, z$ & $4.482(2)$ & $3.5545(14)$ & $-3.5501(15)$ & $1.47(18)$ & 37.6 & 37.5 \\
\hline $\operatorname{Cg}(3) \cdots \operatorname{Cg}(4)$ & $x, 1+y, z$ & $3.834(2)$ & $3.4785(14)$ & $-3.5412(15)$ & $2.48(17)$ & 22.5 & 24.9 \\
\hline $\operatorname{Cg}(4) \operatorname{Cg}(4)$ & $1-x, 1 / 2+y,-z$ & $4.977(2)$ & $-1.4004(15)$ & $-4.8426(15)$ & $79.59(18)$ & 13.3 & 73.7 \\
\hline \multicolumn{8}{|l|}{ (1) } \\
\hline $\mathrm{Cg}(1) \cdots \operatorname{Cg}(4)$ & $1 / 2+x, 1 / 2-y, 2-z$ & $4.5303(8)$ & $2.4145(2)$ & $4.4321(5)$ & $46.41(6)$ & 12.0 & 57.8 \\
\hline & & & group $\boldsymbol{B}$ & & & & \\
\hline \multicolumn{8}{|l|}{ OGIXOT } \\
\hline $\operatorname{Cg}(1) \quad \operatorname{Cg}(2)$ & $x,-1+y, z$ & $3.896(2)$ & $3.5805(17)$ & $-3.6435(16)$ & $2.5(2)$ & 20.7 & 23.2 \\
\hline $\operatorname{Cg}(1) \cdot \operatorname{Cg}(3)$ & $x, 1+y, z$ & $4.560(2)$ & $-3.6222(17)$ & $3.6338(16)$ & $1.9(2)$ & 37.2 & 37.4 \\
\hline $\operatorname{Cg}(2) \quad \operatorname{Cg}(3)$ & $x, 1+y, z$ & $4.432(2)$ & $-3.6559(16)$ & $3.5516(17)$ & $2.46(19)$ & 36.7 & 34.4 \\
\hline \multicolumn{8}{|l|}{ VERXUO } \\
\hline $\mathrm{Cg}(1) \cdots \operatorname{Cg}(2)$ & $-1+x, y, z$ & $4.384(10)$ & $3.477(6)$ & $-3.492(6)$ & $4.1(8)$ & 37.2 & 37.5 \\
\hline $\operatorname{Cg}(1) \cdots \operatorname{Cg}(3)$ & $1+x, y, z$ & $3.838(9)$ & $-3.501(6)$ & $3.556(6)$ & $2.4(7)$ & 22.1 & 24.2 \\
\hline $\operatorname{Cg}(2) \cdots \operatorname{Cg}(3)$ & $1+x, y, z$ & $4.045(9)$ & $-3.409(6)$ & $3.584(6)$ & 5.1(7) & 27.6 & 32.6 \\
\hline $\operatorname{Cg}(2) \cdots \operatorname{Cg}(16)$ & $-1+x,-1+y, 1+z$ & $4.152(16)$ & $1.324(6)$ & $-3.864(15)$ & 53 & 21.5 & 71.4 \\
\hline $\mathrm{Cg}(4) \mathrm{Cg}(10)$ & $x,-1+y, z$ & $4.940(9)$ & $-1.412(6)$ & $-4.225(6)$ & $50.4(7)$ & 31.2 & 73.4 \\
\hline $\operatorname{Cg}(8) \cdots \operatorname{Cg}(9)$ & $-1+x, y, z$ & $4.510(10)$ & $3.554 \backslash(6)$ & $-3.522(6)$ & $3.2(8)$ & 38.7 & 38.0 \\
\hline $\operatorname{Cg}(8) \cdots \operatorname{Cg}(10)$ & $1+x, y, z$ & $3.876(10)$ & $-3.483(6)$ & $3.566(6)$ & 3.1(8) & 23.1 & 26.0 \\
\hline $\operatorname{Cg}(9) \cdots \operatorname{Cg}(3)$ & $1+x, y, z$ & $4.225(9)$ & $-3.395(6)$ & $3.601(6)$ & $5.0(7)$ & 31.5 & 36.5 \\
\hline \multicolumn{8}{|l|}{ OGIXUZ } \\
\hline $\mathrm{Cg}(1) \operatorname{Cg}(1)$ & $x,-1+y, z$ & $4.887(10)$ & $3.572(7)$ & $-3.571(7)$ & 0 & 43.1 & 43.1 \\
\hline $\mathrm{Cg}(1) \cdots \operatorname{Cg}(2)$ & $x, 1+y, z$ & $4.723(11)$ & $-3.517(7)$ & $3.557(8)$ & 1 & 41.1 & 41.9 \\
\hline $\operatorname{Cg}(1) \cdots \operatorname{Cg}(3)$ & $x,-1+y, z$ & 3.934(10) & $3.466(7)$ & $-3.541(7)$ & $2.4(9)$ & 25.8 & 28.3 \\
\hline $\operatorname{Cg}(2) \operatorname{Cg}(2)$ & $x,-1+y, z$ & $4.887(11)$ & $3.499(8)$ & $-3.500(8)$ & $0.0(9)$ & 44.3 & 44.3 \\
\hline $\operatorname{Cg}(2) \cdot \operatorname{Cg}(3)$ & $x,-1+y, z$ & $4.649(10)$ & $3.447(8)$ & $-3.535(7)$ & $1.8(8)$ & 40.5 & 42.2 \\
\hline $\operatorname{Cg}(3) \cdot \operatorname{Cg}(3)$ & $x,-1+y, z$ & $4.887(9)$ & $3.443(7)$ & $-3.442(7)$ & $0.0(8)$ & 45.2 & 45.2 \\
\hline $\mathrm{Cg}(4) \cdots \operatorname{Cg}(4)$ & & $4.887(11)$ & $3.170(7)$ & $-3.170(7)$ & $0.0(8)$ & 49.6 & 49.6 \\
\hline EKEWUM & & & & & $1.3(3)$ & 30.3 & 31.5 \\
\hline $\operatorname{Cg}(1) \cdots \operatorname{Cg}(3)$ & $1+x, y, z$ & $4.065(4)$ & $-3.466(2)$ & $3.510(2)$ & & & \\
\hline $\operatorname{Cg}(1) \operatorname{Cg}(4)$ & $-1+x, y, z$ & $4.507(4)$ & $3.428(2)$ & $-3.495(2)$ & $2.1(3)$ & 39.1 & 40.5 \\
\hline $\operatorname{Cg}(3) \cdots \operatorname{Cg}(4)$ & $-1+x, y, z$ & $3.729(4)$ & $3.478(2)$ & $-3.473(2)$ & $0.9(2)$ & 21.4 & 21.2 \\
\hline $\operatorname{Cg}(4) \cdot \operatorname{Cg}(2)$ & $1 / 2+x, 1 / 2-y, 1-z$ & $4.767(5)$ & $2.285(2)$ & $-3.994(2)$ & $40.6(2)$ & 33.1 & 61.3 \\
\hline \multicolumn{8}{|l|}{ NUBPEH } \\
\hline $\operatorname{Cg}(1) \cdots \operatorname{Cg}(3)$ & $x,-1+y, z$ & $3.806(11)$ & $3.528(7)$ & $-3.589(6)$ & $2.9(8)$ & 19.4 & 22.1 \\
\hline
\end{tabular}




\begin{tabular}{|c|c|c|c|c|c|c|c|}
\hline $\operatorname{Cg}(1) \cdots \operatorname{Cg}(4)$ & $x, 1+y, z$ & $4.275(11)$ & $-3.558(7)$ & $3.578(6)$ & $2.8(8)$ & 33.2 & 33.7 \\
\hline $\operatorname{Cg}(3) \cdots \operatorname{Cg}(4)$ & & $3.914(10)$ & $-3.597(6)$ & $3.495(6)$ & $3.7(7)$ & 26.8 & 23.2 \\
\hline $\operatorname{Cg}(9) \cdots \operatorname{Cg}(12)$ & & $3.883(11)$ & $-3.466(7)$ & $3.534(7)$ & $2.6(8)$ & 24.5 & 26.8 \\
\hline $\mathrm{Cg}(9) \operatorname{Cg}(13)$ & $x,-1+y, z$ & $4.584(11)$ & $3.526(6)$ & $-3.512(6)$ & $1.4(8)$ & 40.0 & 39.7 \\
\hline $\mathrm{Cg}(12) \cdots \operatorname{Cg}(13)$ & & $4.330(11)$ & $3.550(7)$ & $-3.401(6)$ & $3.4(7)$ & 38.2 & 34.9 \\
\hline \multicolumn{8}{|c|}{ group $C$} \\
\hline \multicolumn{8}{|l|}{ DULLAZ } \\
\hline $\mathrm{Cg}(1) \cdots \operatorname{Cg}(4)$ & $1+x, y, z$ & $4.239(10)$ & $-3.490(7)$ & $3.575(7)$ & $3.2(9)$ & 32.5 & 34.6 \\
\hline $\operatorname{Cg}(3) \operatorname{Cg}(4)$ & $1+x, y, z$ & $4.589(10)$ & $-3.348(7)$ & $3.507(7)$ & $5.1(8)$ & 40.2 & 43.1 \\
\hline $\mathrm{Cg}(8) \cdots \mathrm{Cg}(11)$ & $1+x, y, z$ & $4.238(10)$ & $3.426(7)$ & $-3.452(7)$ & $4.8(9)$ & 35.4 & 36.0 \\
\hline $\operatorname{Cg}(10) \cdot \operatorname{Cg}(11)$ & & $4.632(10)$ & $3.186(7)$ & $-3.454(7)$ & $7.1(8)$ & 41.8 & 46.5 \\
\hline \multicolumn{8}{|l|}{ MOXSUP } \\
\hline $\operatorname{Cg}(1) \cdots \operatorname{Cg}(3)$ & $1+x, y, z$ & $4.445(2)$ & $-3.5135(14)$ & $3.5532(14)$ & $1.98(18)$ & 36.9 & 37.8 \\
\hline \multicolumn{8}{|c|}{ group $D$} \\
\hline \multicolumn{8}{|l|}{ WATSIU01 } \\
\hline $\mathrm{Cg}(1) \quad \mathrm{Cg}(20)$ & $2-x,-1 / 2+y, 1-z$ & $4.238(7)$ & $-0.5585(13)$ & $4.093(6)$ & 72 & 15.1 & 82.4 \\
\hline $\operatorname{Cg}(2) \quad \operatorname{Cg}(3)$ & $-1+x, y, z$ & $4.588(2)$ & $1.6614(10)$ & $-4.3791(11)$ & $52.41(12)$ & 17.3 & 68.8 \\
\hline $\mathrm{Cg}(3) \quad \mathrm{Cg}(20)$ & $2-x,-1 / 2+y, 1-z$ & $4.232(5)$ & $-2.2280(12)$ & $4.090(6)$ & 72 & 14.9 & 58.2 \\
\hline $\mathrm{Cg}(8) \quad \operatorname{Cg}(15)$ & $1-x,-1 / 2+y, 1-z$ & $4.136(7)$ & $3.5765(13)$ & $-4.116(7)$ & 35 & 5.7 & 30.2 \\
\hline $\mathrm{Cg}(8) \quad \operatorname{Cg}(20)$ & $1-x,-1 / 2+y, 1-z$ & $4.307(6)$ & $3.8803(13)$ & $-4.187(6)$ & 37 & 13.6 & 25.8 \\
\hline $\operatorname{Cg}(9) \cdot \operatorname{Cg}(10)$ & $1+x, y, z$ & $4.729(2)$ & $1.8484(10)$ & $4.5402(12)$ & $59.85(14)$ & 16.3 & 67.0 \\
\hline $\mathrm{Cg}(10) \cdots \operatorname{Cg}(15)$ & $1-x,-1 / 2+y, 1-z$ & $4.705(9)$ & $3.1056(13)$ & $-4.130(7)$ & 37 & 28.6 & 48.7 \\
\hline $\mathrm{Cg}(10) \cdots \operatorname{Cg}(20)$ & $1-x,-1 / 2+y, 1-z$ & $4.859(8)$ & $4.5646(13)$ & $-4.237(6)$ & 35 & 29.3 & 20.1 \\
\hline $\operatorname{Cg}(11) \cdots \overline{C g}(3)$ & $-1+x, 1+y, z$ & $4.891(2)$ & $-0.0509(12)$ & $4.8376(12)$ & $84.45(13)$ & 8.5 & 89.4 \\
\hline $\operatorname{Cg}(11) \cdots \operatorname{Cg}(15)$ & $1-x,-1 / 2+y, 1-z$ & $4.765(6)$ & $4.6401(12)$ & $-4.108(6)$ & 35 & 30.4 & 13.1 \\
\hline $\mathrm{Cg}(11) \cdots \overline{C g}(20)$ & $1-x,-1 / 2+y, 1-z$ & $4.510(5)$ & $2.6206(12)$ & $-4.193(6)$ & 37 & 21.6 & 54.5 \\
\hline $\operatorname{Cg}(15) \quad \operatorname{Cg}(8)$ & $1-x, 1 / 2+y, 1-z$ & $4.137(7)$ & $-4.116(7)$ & $3.5766(13)$ & 35 & 30.2 & 5.7 \\
\hline $\operatorname{Cg}(15) \cdot \operatorname{Cg}(10)$ & $1-x, 1 / 2+y, 1-z$ & $4.705(9)$ & $-4.130(7)$ & $3.1056(13)$ & 37 & 48.7 & 28.6 \\
\hline $\mathrm{Cg}(15) \cdots \operatorname{Cg}(11)$ & $1-x, 1 / 2+y, 1-z$ & $4.765(6)$ & $-4.108(6)$ & $4.6400(12)$ & 35 & 13.1 & 30.4 \\
\hline $\operatorname{Cg}(20) \quad \operatorname{Cg}(3)$ & $2-x, 1 / 2+y, 1-z$ & $4.232(5)$ & $4.090(6)$ & $-2.2279(12)$ & 72 & 58.2 & 14.9 \\
\hline $\mathrm{Cg}(20) \quad \operatorname{Cg}(8)$ & $1-x, 1 / 2+y, 1-z$ & $4.308(6)$ & $-4.187(6)$ & $3.8802(13)$ & 37 & 25.8 & 13.6 \\
\hline $\operatorname{Cg}(20) \cdot \operatorname{Cg}(10)$ & $1-x, 1 / 2+y, 1-z$ & $4.859(8)$ & $-4.237(6)$ & $4.5646(13)$ & 35 & 20.1 & 29.3 \\
\hline $\operatorname{Cg}(20) \cdots \operatorname{Cg}(11)$ & $1-x, 1 / 2+y, 1-z$ & $4.510(5)$ & $-4.194(6)$ & $2.6206(12)$ & 37 & 54.5 & 21.6 \\
\hline \multicolumn{8}{|l|}{ UQOGUE } \\
\hline $\operatorname{Cg}(4) \cdot \operatorname{Cg}(6)$ & $-1 / 2+x,-1 / 2-y,-z$ & $4.802(5)$ & $-2.514(3)$ & $-4.686(3)$ & $60.5(3)$ & 12.6 & 58.4 \\
\hline $\operatorname{Cg}(5) \cdots \operatorname{Cg}(6)$ & $-1 / 2+x,-1 / 2-y,-z$ & $4.800(4)$ & $-1.924(3)$ & $-4.672(3)$ & $59.7(3)$ & 13.3 & 66.4 \\
\hline $\operatorname{Cg}(6) \cdots \operatorname{Cg}(5)$ & $-1 / 2+x,-1 / 2-y,-z$ & $4.938(5)$ & $-1.788(3)$ & $-4.756(3)$ & 59.7(3) & 15.6 & 68.8 \\
\hline \multicolumn{8}{|l|}{ UQIYUG } \\
\hline $\operatorname{Cg}(1) \cdot \operatorname{Cg}(3)$ & $-1 / 2+x,-1 / 2-y,-z$ & $4.8990(14)$ & $-2.4542(9)$ & $-4.7341(8)$ & $63.20(11)$ & 12.5 & 59.6 \\
\hline $\mathrm{Cg}(2) \cdots \operatorname{Cg}(3)$ & $-1 / 2+x,-1 / 2-y,-z$ & $4.8930(14)$ & $-1.8807(8)$ & $-4.7251(8)$ & $61.78(10)$ & 13.7 & 67.2 \\
\hline $\operatorname{Cg}(3) \cdot \operatorname{Cg}(2)$ & $-1 / 2+x,-1 / 2-y,-z$ & $4.9595(14)$ & $-1.8386(8)$ & $-4.8209(8)$ & $61.78(10)$ & 13.6 & $-1.8386(8)$ \\
\hline \multicolumn{8}{|c|}{ group $E$} \\
\hline XATKEL & & & & & & & \\
\hline $\mathrm{Cg}(1) \cdots \operatorname{Cg}(1)$ & $x, y,-1+z$ & $4.997(14)$ & $-3.456(9)$ & $3.454(9)$ & 0 & 46.3 & 46.3 \\
\hline $\operatorname{Cg}(1) \cdots \operatorname{Cg}(3)$ & $x, y, 1+z$ & $3.809(13)$ & 3.471(9) & $-3.478(8)$ & 2 & 24.1 & 24.3 \\
\hline $\mathrm{Cg}(1) \cdot \operatorname{Cg}(4)$ & $x, y,-1+z$ & $4.422(11)$ & $-3.419(4)$ & $3.452(4)$ & $0.7(9)$ & 38.7 & 39.4 \\
\hline $\operatorname{Cg}(2) \cdots \operatorname{Cg}(2)$ & $x, y,-1+z$ & $4.999(12)$ & $3.115(7)$ & $-3.117(7)$ & $0.0(8)$ & 51.5 & 51.5 \\
\hline $\mathrm{Cg}(3) \cdots \operatorname{Cg}(3)$ & $x, y,-1+z$ & $4.999(14)$ & $-3.483(8)$ & $3.483(8)$ & 0 & 45.8 & 45.8 \\
\hline $\operatorname{Cg}(3) \cdots \operatorname{Cg}(4)$ & $x, y,-1+z$ & $4.079(9)$ & $-3.450(8)$ & $3.395(4)$ & $1.7(8)$ & 33.7 & 32.2 \\
\hline $\operatorname{Cg}(4) \quad \operatorname{Cg}(4)$ & $x, y, 1+z$ & 4.999(6) & $3.432(4)$ & $-3.432(4)$ & $0.0(5)$ & 46.6 & 46.6 \\
\hline ХАТJАG & & & & & & & \\
\hline $\mathrm{Cg}(1) \cdot \operatorname{Cg}(1)$ & $x, y,-1+z$ & $4.998(8)$ & $3.526(5)$ & $-3.525(5)$ & $0.0(6)$ & 45.1 & 45.1 \\
\hline $\operatorname{Cg}(1) \cdots \operatorname{Cg}(3)$ & $x, y, 1+z$ & $3.832(7)$ & $3.483(5)$ & $3.519(4)$ & $1.6(6)$ & 23.3 & 24.7 \\
\hline $\operatorname{Cg}(1) \quad \operatorname{Cg}(4)$ & $x, y,-1+z$ & $4.469(6)$ & $-3.417(5)$ & $-3.482(2)$ & $2.2(5)$ & 38.8 & 40.1 \\
\hline $\operatorname{Cg}(2) \cdot \operatorname{Cg}(2)$ & $x, y,-1+z$ & $4.997(6)$ & $3.084(4)$ & $-3.084(4)$ & $0.0(5)$ & 51.9 & $3.084(4)$ \\
\hline $\mathrm{Cg}(3) \cdot \operatorname{Cg}(3)$ & $x, y,-1+z$ & 4.997(7) & $-3.467(4)$ & $3.467(4)$ & $0.0(5)$ & 46.1 & 46.1 \\
\hline $\operatorname{Cg}(3) \quad \operatorname{Cg}(4)$ & $x, y,-1+z$ & $4.152(5)$ & $-3.494(4)$ & $3.421(2)$ & $2.2(4)$ & 34.5 & 32.7 \\
\hline $\operatorname{Cg}(4) \cdot \operatorname{Cg}(4)$ & $x, y,-1+z$ & 4.997(3) & $-3.393(2)$ & $3.393(2)$ & $0.0(3)$ & 47.2 & 47.2 \\
\hline ХАТЈЕК & & & & & & & \\
\hline $\operatorname{Cg}(1) \cdot \operatorname{Cg}(1)$ & $x,-1+y, z$ & $4.839(6)$ & $3.708(4)$ & $-3.708(4)$ & $0.0(5)$ & 40.0 & 40.0 \\
\hline $\operatorname{Cg}(1) \cdots \operatorname{Cg}(3)$ & $x, 1+y, z$ & $3.936(6)$ & $-3.648(4)$ & $3.706(4)$ & $2.6(5)$ & 19.7 & 22.1 \\
\hline $\operatorname{Cg}(1) \cdot \operatorname{Cg}(4)$ & $x,-1+y, z$ & $4.572(6)$ & $3.632(4)$ & $-3.677(4)$ & $1.8(5)$ & 36.5 & 37.4 \\
\hline $\operatorname{Cg}(2) \cdot \operatorname{Cg}(2)$ & $x,-1+y, z$ & $4.839(6)$ & $3.326(4)$ & $-3.326(4)$ & $0.0(5)$ & 46.6 & 46.6 \\
\hline $\operatorname{Cg}(3) \cdots \operatorname{Cg}(3)$ & $x,-1+y, z$ & $4.839(6)$ & $3.638(4)$ & $-3.638(4)$ & $0.0(5)$ & 41.3 & 41.3 \\
\hline $\operatorname{Cg}(3) \cdots \operatorname{Cg}(4)$ & $x,-1+y, z$ & $4.555(6)$ & $3.701(4)$ & $-3.567(4)$ & $2.8(5)$ & 38.4 & 35.7 \\
\hline $\mathrm{Cg}(4) \cdots \operatorname{Cg}(4)$ & $x,-1+y, z$ & $4.839(6)$ & $3.610(4)$ & $-3.610(4)$ & $0.0(5)$ & 41.8 & 41.8 \\
\hline XATKIP & & & & & & & \\
\hline $\mathrm{Cg}(1) \cdots \operatorname{Cg}(1)$ & $-1+x, y, z$ & $4.920(7)$ & $3.510(4)$ & $-3.511(4)$ & $0.0(6)$ & 44.5 & 44.5 \\
\hline $\operatorname{Cg}(1) \quad \operatorname{Cg}(2)$ & $1+x, y, z$ & $4.441(6)$ & $-3.476(4)$ & $3.424(4)$ & $3.9(5)$ & 39.6 & 38.5 \\
\hline $\operatorname{Cg}(1) \cdots \operatorname{Cg}(3)$ & $-1+x, y, z$ & $3.821(6)$ & $3.482(4)$ & $-3.516(3)$ & $2.1(5)$ & 23.0 & 24.3 \\
\hline $\operatorname{Cg}(2) \quad \operatorname{Cg}(2)$ & $-1+x, y, z$ & $4.920(6)$ & $3.417(4)$ & $-3.418(4)$ & $0.0(4)$ & 46.0 & 46.0 \\
\hline $\operatorname{Cg}(2) \quad \operatorname{Cg}(3)$ & $-1+x, y, z$ & $4.221(5)$ & $3.336(4)$ & $-3.576(3)$ & $5.8(4)$ & 32.1 & 37.8 \\
\hline $\operatorname{Cg}(3) \cdots \operatorname{Cg}(3)$ & $-1+x, y, z$ & $4.920(5)$ & $3.489(3)$ & $-3.489(3)$ & $0.0(4)$ & 44.8 & 44.8 \\
\hline $\operatorname{Cg}(4) \cdot \operatorname{Cg}(4)$ & $-1+x, y, z$ & $4.920(5)$ & $3.202(4)$ & $-3.202(4)$ & $0.0(4)$ & 49.4 & 49.4 \\
\hline
\end{tabular}

${ }^{a, b}$ In (1): $\boldsymbol{C g}(\mathbf{1})$ is the centroid of the 5-membered ring of the Fmoc system (C16/C17/C22/C23/C28) and Cg(4)-6-membered ring of the Fmoc group (C23/C24/C25/C26/C27/C28) [symmetry code: 1/2+x,1/2-y, 2-z], in OGIXOT: Cg1 and Cg2 are the centroids of the 
CAMLEK: Cg1: C1-C2-C7-C8-C13; Cg2: C2-C3-C4-C5-C6-C7; Cg3: C8-C9-C10-C11-C12-C13; Cg4: C19-C20-C21-C22-C23-C(24); OGOGIA: Cg1: C1C6-C7-C12-C13; Cg2: C1-C2-C3-C4-C5-C6; Cg3: C7-C8-C9-C10-C11- C12; Cg4: C20-C21-C22-C23-C24-C25; OGIYAG: Cg1: C1-C6-C7-C12-C13; Cg 2: C1-C2-C3-C4-C5-C6; Cg 3: C7-C8-C9-C10-C11-C12; Cg 4: C19-C20-C21-C22-C23-C(24); INEJEQ: Cg1: C18-C19-C24-C25-C30; Cg2: C1-C2-C3-C4-C5C6; Cg3: C19-C20-C21-C22-C23-C24; Cg4: C25-C26-C27-C28-C29-C30; VERXUO: Cg1: C1-C2-C7-C8-C13; Cg2: C2-C3-C4-C5-C6-C7; Cg3: C8-C9-C10C11-C12-C13; Cg4: C19-C20-C21-C22-C23-C24; Cg8: C25-C26-C31-C32-C37; Cg9:C26-C27-C28-C29-C30-C31; Cg10: C32-C33-C34-C35-C36-C37; Cg16: S2-S3-C52-C54; OGIXUZ: Cg1: C1-C6-C7-C12-C13; Cg2: C1-C2-C3-C4-C5-C6; Cg3: C7-C8-C9-C10-C11-C12; Cg4: C19-C20-C21-C22-C23-C24; EKEWUM: Cg1: C12-C13-C14-C20-C19; Cg2: N1-C1-C5-C4-C3-C2; Cg3: C13-C14-C15-C16-C17-C18; Cg4: C19-C20-C21-C22-C23-C24; NUBPEH: Cg1: C14-C15-C20-C21-C26; Cg2: C1-C2-C3-C4-C5-C6; Cg3: C15-C16-C17-C18-C19-C20; Cg4: C21-C22-C23-C24-C25-C26; Cg5: C28-C29-C30-C31-C32-C33; Cg9: C54-C55-C60-C61-C66; Cg10: C34-C35-C36-C37-C38-C39; Cg11: C46-C47-C48-C49-C50-C51; Cg12: C55-C56-C57-C58-C59-C60; Cg13: C61-C62C63-C64-C65-C66; DULLAZ: Cg1: C16-C17-C22-C23-C28; Cg2: C5-C6-C7-C8-C9-C10; Cg3: C17-C18-C19-C20-C21-C22; Cg4: C23-C24-C25-C26-C27C28; Cg8: C44-C45-C50-C51-C56; Cg9: C33-C34-C35-C36-C37-C38; Cg10: C45-C46-C47-C48-C49-C50; Cg11: C51-C52-C53-C54-C55-C56; MOXSUP: Cg1: C3-C4-C9-C10-C15; Cg2: C4-C5-C6-C7-C8-C9; Cg3: C10-C11-C12-C13-C14-C15; Cg4: C19-C20-C21-C22-C23-C24; Cg5: C26-C27-C28-C29-C30C31; WATSIU01: Cg1: C12-C13-C18-C19-C24; Cg2: C4-C5-C6-C7-C8-C9; Cg3: C13-C14-C15-C16-C17-C18; Cg4: C19-C20-C21-C22-C23-C24; Cg8: C36C37-C42-C43-C48; Cg9: C28-C29-C30-C31-C32-C33; Cg10: C37-C38-C39-C40-C41-C42; Cg11: C43-C44-C45-C46-C47-C48; Cg15: Cl3-C15-C50-C51; Cg16: Cl3-C15-C51-C50; Cg20: C14-Cl6-C50-C51; UQOGUE Cg4: C1-C2-C7-C8-C13; Cg5: C2-C3-C4-C5-C6-C7; Cg6: C8-C9-C10-C11-C12-C13; Cg7: C19C20-C21-C22-C23-C24; UQIYUG: Cg1: C1-C2-C7-C8-C13; Cg2: C2-C3-C4-C5-C6-C7; Cg3: C8-C9-C10-C11-C12-C13; Cg4: C19-C20-C21-C22-C23-C24; XATKEL Cg1: C7-C12-C13-C18-C19; Cg2: C1-C2-C3-C4-C5-C6; Cg3: C7-C8-C9-C10-C11-C12; Cg4: C13-C14-C15-C16-C17-C18; XATJAG Cg1: C7-C12C13-C18-C19; Cg2: C1-C2-C3-C4-C5-C6; Cg3: C7-C8-C9-C10-C11-C12; Cg4: C13-C14-C15-C16-C17-C18; XATJEK: Cg1: C5-C10-C6-C14-C24; Cg2: C2C4-C8-C3-C15-C11; Cg3: C5-C7-C16-C17-C19-C24; Cg4: C6-C14-C23-C22-C20-C18; XATKIP Cg1: C1-C6-C7-C12-C13; Cg2: C1-C2-C3-C4-C5-C6; Cg3: C7-C8-C9-C10-C11-C12; Cg4: C19-C20-C21-C22-C23-C24.

\section{Table S6}

Geometrical parameters for $\mathrm{C}-\mathrm{H}^{\cdots} \pi$ inter-contacts.

${ }^{a} \mathrm{Cg}(\mathrm{J})$ - center of gravity of ring $\mathrm{J} ; \mathrm{H}^{\cdots} \mathrm{Cg},{ }^{b} \mathrm{X}^{\cdots} \mathrm{Cg}$ - distance $[A],{ }^{c} \mathrm{X}-\mathrm{H}^{\cdots} \mathrm{Cg}$ - angle $\left[{ }^{o}\right],{ }^{d} \gamma$ - angle between $\mathrm{Cg}$ - $\mathrm{H}$ vector and ring $\mathrm{J}$ normal, ${ }^{e}$ $H$-perp-perpendicular distance of $H$ to ring plane $J,{ }^{f} X-H, \pi$-angle of the $X-H$ bond with the $\pi$-plane $\left(\right.$ perpendicular $=90^{\circ}$, parallel $\left.=0^{\circ}\right)$.

\begin{tabular}{|c|c|c|c|c|c|c|c|}
\hline $\mathrm{X}^{\cdots \cdot} \mathrm{H}(\mathrm{Y}-\mathrm{X})-\mathrm{Cg}(\mathrm{J})^{\mathrm{a}}$ & symmetry & $\mathrm{H}(\mathrm{X})^{\cdots} \mathrm{Cg}$ & $\mathrm{X}(\mathrm{Y})^{\cdots \cdot \mathrm{Cg}^{\mathrm{b}}}$ & $\mathrm{X}-\mathrm{H}(\mathrm{Y}-\mathrm{X})^{\cdots} \mathrm{Cg}^{\mathrm{c}}$ & $\gamma^{\mathrm{d}}$ & $\begin{array}{l}\mathrm{H}(\mathrm{X})- \\
\text { perp }^{\text {e }}\end{array}$ & $\mathrm{X}-\mathrm{H}(\mathrm{Y}-\mathrm{X}), \pi^{\mathrm{f}}$ \\
\hline group & & & & & & & \\
\hline \multicolumn{8}{|c|}{ CAMLEK } \\
\hline $\mathrm{C}(9)-\mathrm{H}(10) \cdots \mathrm{Cg}(2)$ & $1-x, 1 / 2+y,-z$ & 2.80 & $3.590(5)$ & 142 & 13.12 & -2.72 & 56 \\
\hline $\mathrm{C} 15-\mathrm{O} 2 \cdots \mathrm{Cg}(1)$ & $x,-1+y, z$ & $3.804(4)$ & $383(5)$ & $110.7(3)$ & 3.87 & 3.795 & 16.79 \\
\hline \multicolumn{8}{|l|}{ OGIYAG } \\
\hline $\mathrm{C}(4)-\mathrm{H}(6) \cdots \mathrm{Cg}(3)$ & $1 / 2-x, 1 / 2+y, 1-z$ & $2.93(3)$ & $3.546(4)$ & 121(2) & 14.76 & 2.84 & 46 \\
\hline $\mathrm{C}(5)-\mathrm{H}(7) \cdots \mathrm{Cg}(1)$ & $1 / 2-x, 1 / 2+y, 1-z$ & $2.92(2)$ & $3.567(4)$ & $126.9(19)$ & 23.37 & 2.68 & 52 \\
\hline $\mathrm{C}(8)-\mathrm{H}(8) \cdots \mathrm{Cg}(2)$ & $1 / 2-x, 1 / 2+y, 1-z$ & $2.91(3)$ & $3.782(4)$ & $148(2)$ & 6.80 & 2.89 & 58 \\
\hline $\mathrm{C} 15-\mathrm{O} 2 \cdots \mathrm{Cg} 1$ & $x,-1+y, z$ & $3.339(3)$ & $4.250(4)$ & $131.68(13)$ & 12.51 & -3.259 & 30.97 \\
\hline $\mathrm{C} 15-\mathrm{O} 2{ }^{\prime} \mathrm{Cg} 2$ & $x,-1+y, z$ & $3.643(4)$ & $3.946(4)$ & $95.19(12)$ & 26.30 & -3.266 & 30.73 \\
\hline \multicolumn{8}{|l|}{ INEJEQ } \\
\hline $\mathrm{C} 4-\mathrm{H} 2 \cdots \mathrm{Cg} 2$ & $1-x,-1-2+y, 1-z$ & 2.82 & $3.612(3)$ & 141 & 16.92 & 2.70 & 57 \\
\hline $\mathrm{C} 7-\mathrm{H} 4 \mathrm{Cg} 2$ & $x, 1+y, z$ & 2.89 & $3.737(4)$ & 145 & 6.09 & -2.88 & 54 \\
\hline \multicolumn{8}{|l|}{ (1) } \\
\hline $\mathrm{C} 29-\mathrm{H} 29 \mathrm{~A} \cdots \mathrm{Cg}(4)$ & $1+x, y, z$ & 2.91 & $3.5457(15)$ & 124 & 6.87 & -2.89 & 41 \\
\hline $\mathrm{C} 24-\mathrm{H} 241 \cdots \mathrm{Cg}(1)$ & $-1 / 2+x, 1 / 2-y, 2-z$ & $2.957(19)$ & $3.4952(13)$ & $114.8(13)$ & 17.58 & 2.82 & 42 \\
\hline
\end{tabular}
group $B$

\section{OGIXOT}

\begin{tabular}{|c|c|c|c|c|c|c|c|}
\hline $\mathrm{C} 13-\mathrm{H} 15 \cdots \mathrm{Cg}(2)$ & $x,-1+y, z$ & $2.92(4)$ & $3.695(4)$ & 134(3) & 13.26 & 2.84 & 53 \\
\hline \multicolumn{8}{|l|}{ VERXUO } \\
\hline $\mathrm{C} 25-\mathrm{H} 24 \cdots \mathrm{Cg} 10$ & $1+x, y, z$ & 2.97 & $3.680(16)$ & 128 & 17.96 & -2.83 & 49 \\
\hline C36-H32 ․ Cg4 & $x, 1+y, z$ & 2.84 & $3.660(16)$ & 145 & 15.39 & -2.74 & 40 \\
\hline $\mathrm{C} 50-\mathrm{H} 46 \cdots \mathrm{Cg} 9$ & $x,-1+y, 1+z$ & 2.88 & $3.802(19)$ & 157 & 15.64 & -2.78 & 53 \\
\hline $\mathrm{C} 15-\mathrm{O} 2 \cdots \mathrm{Cg} 4$ & $x, y, z$ & $3.949(12)$ & $4.030(15)$ & $85.2(8)$ & 25.7 & 3.558 & 22.74 \\
\hline \multicolumn{8}{|l|}{ OGIXUZ } \\
\hline $\mathrm{C} 13-\mathrm{H} 11^{\cdots} \mathrm{Cg} 3$ & $x,-1+y, z$ & 2.77 & $3.583(16)$ & 139 & 5.37 & 2.76 & 50 \\
\hline \multicolumn{8}{|l|}{ EKEWUM } \\
\hline $\mathrm{C} 5-\mathrm{H} 4 \cdots \mathrm{Cg} 4$ & $-1 / 2+x, 1 / 2-y, 1-z$ & 2.95 & $3.639(7)$ & 131 & 17.82 & -2.81 & 31 \\
\hline \multicolumn{8}{|l|}{ NUBPEH } \\
\hline $\mathrm{C} 45-\mathrm{H} 44 \cdots \mathrm{Cg} 11$ & $x, 1+y, z$ & 2.72 & $3.425(17)$ & 128 & 10.49 & & \\
\hline $\mathrm{C} 54-\mathrm{H} 52 \cdots \mathrm{Cg} 12$ & & 2.85 & $3.625(17)$ & 135 & 15.49 & & \\
\hline \multicolumn{8}{|c|}{ group $C$} \\
\hline C44-H50 ․ Cg11 & $1+x, y, z$ & 2.89 & $3.738(17)$ & 143 & 18.21 & 2.75 & 49 \\
\hline $\mathrm{C} 53-\mathrm{H} 56 \cdots \mathrm{Cg} 3$ & $-x, 1 / 2+y, 1-z$ & 2.95 & $3.766(19)$ & 145 & 23.13 & -2.71 & 78 \\
\hline $\mathrm{C} 2-\mathrm{O} 1 \cdots \mathrm{Cg} 2$ & $x, y, z$ & $3.796(13)$ & $3.659(18)$ & $74.4(10)$ & 28.91 & 3.323 & 43.54 \\
\hline \multicolumn{8}{|l|}{ MOXSUP } \\
\hline $\mathrm{C} 3-\mathrm{H} 5 \cdots \mathrm{Cg} 3$ & $1+x, y, z$ & 2.90 & $3.847(4)$ & 158 & 16.60 & -2.78 & 52 \\
\hline \multicolumn{8}{|c|}{ WATSIU01 ${ }^{\operatorname{group} D}$} \\
\hline $\mathrm{C} 15-\mathrm{H} 15 \quad \mathrm{Cg} 2$ & $1+x, y, z$ & 2.63 & $3.362(3)$ & 135 & 4.56 & -2.62 & 48 \\
\hline
\end{tabular}




\begin{tabular}{|c|c|c|c|c|c|c|c|}
\hline C17-H17 Cg11 & $1+x,-1+y, z$ & 2.74 & $3.576(3)$ & 147 & 11.49 & 2.69 & 66 \\
\hline C53-H51 Cg4 & $1-x, 1 / 2+y, 1-z$ & 2.87 & $3.839(5)$ & 167 & 14.19 & 2.78 & 68 \\
\hline C49-Cl1 Cg1 & $-1+x, 1+y, z$ & $3.9229(19)$ & $4.531(4)$ & $98.27(12)$ & 16.58 & -3.760 & 8.24 \\
\hline \multicolumn{8}{|l|}{ UQOGUE } \\
\hline C6-H7 Cg6 & $1 / 2+x,-1 / 2-y,-z$ & 2.87 & $3.666(7)$ & 142 & 11.40 & -2.81 & 56 \\
\hline $\mathrm{C} 15-\mathrm{O} 2 \mathrm{Cg} 4$ & $-1+x, y, z$ & $3.315(5)$ & $4.140(7)$ & $125.8(4)$ & 6.66 & 3.292 & 29.68 \\
\hline \multicolumn{8}{|l|}{ UQIYUG } \\
\hline $\mathrm{C} 6-\mathrm{H} 7 \quad \mathrm{Cg} 3$ & $1 / 2+x,-1 / 2-y,-z$ & $2.90(3)$ & $3.686(2)$ & $140(2)$ & 12.70 & -2.83 & 57 \\
\hline $\mathrm{C} 14-\mathrm{H} 12 \mathrm{Cg} 4$ & $-x,-1 / 2+y, 1 / 2-z$ & $2.98(2)$ & $3.872(2)$ & $153(2)$ & 15.27 & 2.88 & 72 \\
\hline $\mathrm{C} 15-\mathrm{O} 2 \mathrm{Cg} 1$ & $-1+x, y, z$ & $3.2499(18)$ & $4.179(2)$ & $133.84(13)$ & 8.76 & 3.212 & 35.16 \\
\hline $\mathrm{C} 15-\mathrm{O} 2 \quad \mathrm{Cg} 2$ & $-1+x, y, z$ & $3.6671(18)$ & $4.013(2)$ & $97.74(12)$ & 28.79 & 3.214 & 34.96 \\
\hline \multicolumn{8}{|c|}{ group $\boldsymbol{E}$} \\
\hline C19-H14 Cg3 & $x, y, 1+z$ & 2.98 & $3.66(3)$ & 126 & 22.84 & 2.75 & 52 \\
\hline $\mathrm{C} 20-\mathrm{O} 2 \mathrm{Cg} 2$ & $x, y, z$ & $3.95(2)$ & $3.901(14)$ & $79.2(12)$ & 21.56 & -3.670 & 22.98 \\
\hline \multicolumn{8}{|l|}{ XАTJAG } \\
\hline C19-H14 Cg3 & $x, y, 1+z$ & 2.98 & $3.659(19)$ & 126 & 21.48 & 2.77 & 51 \\
\hline $\mathrm{C} 20-\mathrm{O} 2 \mathrm{Cg} 2$ & $x, y, z$ & $3.976(12)$ & $3.915(6)$ & $78.3(6)$ & 21.50 & -3.699 & 24.33 \\
\hline \multicolumn{8}{|l|}{ XАТJEK } \\
\hline $\mathrm{C} 10-\mathrm{H} 6 \mathrm{Cg} 3$ & $x, 1+y, z$ & 3.00 & $3.738(10)$ & 132 & 10.50 & -2.95 & 51 \\
\hline \multicolumn{8}{|l|}{ XATKIP } \\
\hline C13-H9 Cg3 & $-1+x, y, z$ & 2.85 & $3.598(14)$ & 132 & 16.07 & 2.74 & 53 \\
\hline $\mathrm{C} 26-\mathrm{H} 29 \mathrm{Cg} 2$ & $-x,-1 / 2+y, 1 / 2-z$ & $2.913 .874(11)$ & $3.70(3)$ & 139 & 18.25 & 2.76 & 51 \\
\hline $\mathrm{C} 15-\mathrm{O} 2 \mathrm{Cg} 4$ & $x, y, z$ & $3.874(11)$ & $3.967(15)$ & $85.5(8)$ & 24.15 & 3.535 & 23.89 \\
\hline
\end{tabular}

\section{Table S7}

Supramolecular H-bonding patterns in the crystals of Fmoc-AAs (taking into account strong H-bonds, Etter, 91; Bernstein, 95).

\begin{tabular}{|c|c|c|}
\hline \multicolumn{3}{|r|}{ Fmoc-Tyr/Phe derivatives } \\
\hline \multicolumn{3}{|r|}{ group $\mathrm{A}$} \\
\hline \multirow[t]{12}{*}{ (1) } & $S(16)$ & $(\mathrm{CHcycl}) \mathrm{C} 26-\mathrm{H} 261 \cdots \mathrm{O} 1_{(-\mathrm{O}-)}$ \\
\hline & $\mathrm{C}(4)$ & (CH) $\mathrm{C} 12-\mathrm{H} 121 \cdots \mathrm{O} 2_{(\mathrm{C}=\mathrm{O})}$ \\
\hline & $\mathrm{C}(5)$ & ${ }_{(\mathrm{CH} 3)} \mathrm{C} 29-\mathrm{H} 29 \mathrm{C} \cdots \mathrm{O} 4_{(\mathrm{C}=\mathrm{O})}$ \\
\hline & $\mathrm{C}(7)$ & (OH) $\mathrm{O} 3-\mathrm{H} 3_{\text {carbonyl }} \cdot \mathrm{O} 4_{(\mathrm{C}=\mathrm{O}) \text { carbonylFMOC }}$ \\
\hline & $\mathrm{C}_{2}^{1}(8)$ & ${ }_{(\mathrm{OH})} \mathrm{O} 3-\mathrm{H} 3_{\text {carbonyl }}{ }^{-\mathrm{O}} 4_{(\mathrm{C}=\mathrm{O}) \text { carbonylFMOC \& (CH3) }} \mathrm{C} 29-\mathrm{H} 29 \mathrm{C}^{\cdots} \mathrm{O} 4_{(\mathrm{C}=\mathrm{O})}$ \\
\hline & $\mathrm{C}(12)$ & (СH3) $\mathrm{C} 4-\mathrm{H} 4 \mathrm{~B} \cdots 3_{(\mathrm{OH})}$ \\
\hline & $\mathrm{C}_{2}^{2}(11)$ & $\begin{array}{l}{ }^{*(\mathrm{OH})} \mathrm{O} 3-\mathrm{H} 3 \cdots \mathrm{O} 4_{(\mathrm{C}=\mathrm{O})} \&(\mathrm{CH}) \mathrm{C} 12-\mathrm{H} 121 \cdots \mathrm{O} 2_{(\mathrm{C}=\mathrm{O})} \\
{ }^{*(\mathrm{CH} 3)} \mathrm{C} 29-\mathrm{H} 29 \mathrm{C}^{\cdots \cdots} 4_{(\mathrm{C}=\mathrm{O})} \&{ }_{(\mathrm{CH})} \mathrm{C} 12-\mathrm{H} 121 \cdots \mathrm{O} 2_{(\mathrm{C}=\mathrm{O})}\end{array}$ \\
\hline & $\mathrm{C}_{2}^{2}(12)$ & ${ }_{(\mathrm{OH})} \mathrm{O} 3-\mathrm{H} 3 \cdots \mathrm{O} 4_{(\mathrm{C}=\mathrm{O})} \&{ }_{(\mathrm{CH} 3)} \mathrm{C} 29-\mathrm{H} 29 \mathrm{C} \cdots \mathrm{O} 4_{(\mathrm{C}=\mathrm{O})}$ \\
\hline & $\mathrm{C}_{2}^{2}(14)$ & (CH3) $\mathrm{C} 4-\mathrm{H} 4 \mathrm{~B} \cdots \mathrm{O} 3_{(\mathrm{OH})} \&(\mathrm{CH}) \mathrm{C} 12-\mathrm{H} 121 \cdots \mathrm{O} 2_{(\mathrm{C}=\mathrm{O})}$ \\
\hline & $\mathrm{C}_{2}^{2}(15)$ & $(\mathrm{OH}) \mathrm{O} 3-\mathrm{H} 3 \cdots{ }^{2} 4_{(\mathrm{C}=\mathrm{O})} \&(\mathrm{CH} 3) \mathrm{C} 4-\mathrm{H} 4 \mathrm{~B} \cdots \mathrm{O} 3_{(\mathrm{OH})}$ \\
\hline & $\mathrm{C}_{2}^{2}(16)$ & $\left.{ }_{(\mathrm{CH} 3)} \mathrm{C} 4-\mathrm{H} 4 \mathrm{~B} \cdots \mathrm{O} 3_{(\mathrm{OH})} \&(\mathrm{CH})\right) \mathrm{C12}-\mathrm{H} 121 \cdots \mathrm{O} 2_{(\mathrm{C}=\mathrm{O})}$ \\
\hline & $\mathrm{C}_{2}^{2}(19)$ & 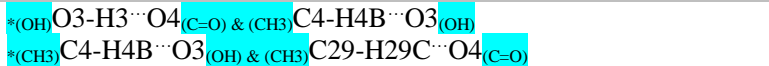 \\
\hline \multirow[t]{4}{*}{ level 2} & $\mathrm{R}_{2}^{2}(9)$ & ${ }_{(\mathrm{OH})} \mathrm{O} 3-\mathrm{H} 3 \cdots \mathrm{O} 4_{(\mathrm{C}=\mathrm{O})} \&(\mathrm{CH})$ \\
\hline & $\mathrm{R}_{3}^{2}(12)$ & ${ }_{(\mathrm{OH})} \mathrm{O} 3-\mathrm{H} 3 \cdots \mathrm{O} 4_{(\mathrm{C}=\mathrm{O})} \&{ }_{(\mathrm{CH} 3)} \mathrm{C} 29-\mathrm{H} 29 \mathrm{C} \cdots \mathrm{O} 4_{(\mathrm{C}=\mathrm{O})}$ \\
\hline & $\mathrm{R}_{3}^{3}(15)$ & ${ }_{(\mathrm{CH})} \mathrm{C} 12-\mathrm{H} 121 \cdots \mathrm{O} 2_{(\mathrm{C}=\mathrm{O})} \&(\mathrm{CH} 3) \mathrm{C} 29-\mathrm{H} 29 \mathrm{C} \cdots \mathrm{O} 4_{(\mathrm{C}=\mathrm{O})}$ \\
\hline & $\mathrm{R}_{4}^{3}(20)$ & ${ }_{(\mathrm{OH})} \mathrm{O} 3-\mathrm{H} 3 \cdots \mathrm{O} 4_{(\mathrm{C}=\mathrm{O})} \&{ }_{(\mathrm{CH} 3)} \mathrm{C} 29-\mathrm{H} 29 \mathrm{C} \cdots \mathrm{O} 4_{(\mathrm{C}=\mathrm{O})}$ \\
\hline CAMLEK & $\mathrm{C}(4)$ & (OH) $\mathrm{O}_{\text {carboxyl }}-\mathrm{H} 1 \cdots \mathrm{O} 4_{(\mathrm{C}=\mathrm{O}) \text { carbonyl }}$ \\
\hline \multirow[t]{4}{*}{ level 1} & $\mathrm{C}(5)$ & $\begin{array}{l}\mathrm{N} 1-\mathrm{H} 4 \cdots \mathrm{O} 3_{\text {carboxyl }} \\
\mathrm{C} 14-\mathrm{H} 15 \cdots \mathbf{O}_{\text {carbonylFMo }} \\
\mathrm{C} 16-\mathrm{H} 16 \cdots \mathbf{O 1}_{\text {carbonylFMo }}\end{array}$ \\
\hline & $\mathrm{C}(10)$ & (OH) $\mathrm{O} 5_{\text {hydroxyl }}-\mathrm{H} 2 \cdots \mathbf{O} 2_{(\mathrm{C}=\mathbf{0}) \text { carbonylFMOC }}$ \\
\hline & $\mathrm{C}(11)$ & (CH2) $^{\mathrm{C} 14-\mathrm{H} 14 \cdots \mathrm{O} 5}(\mathrm{OH})$ \\
\hline & $\mathrm{C}_{2}^{1}(13)$ & $\mathrm{O}_{\text {hydroxyl }}-\mathrm{H} 2 \cdots \mathbf{O} 2_{\text {carbonylFMOC \& }} \mathrm{C} 14-\mathrm{H} 15 \cdots \mathbf{O} 2_{\text {carbonylFMOC }}$ \\
\hline \multirow[t]{9}{*}{ level 2} & $\mathrm{C}_{2}^{2}(7)$ & $\mathrm{O} 3_{\text {carboxyl }}-\mathrm{H} 1 \cdots \mathrm{O} 4_{\text {carbonyl \& }} \mathrm{N} 1-\mathrm{H} 4 \cdots \mathrm{O} 3_{\text {carboxyl }}$ \\
\hline & $\mathrm{C}_{2}^{2}(9)$ & $\mathrm{O} 3_{\text {carboxyl }}-\mathrm{H} 1 \cdots \mathrm{O} 4_{\text {carbonyl \& }} \mathrm{N} 1-\mathrm{H} 4 \cdots \mathrm{O} 3_{\text {carboxyl }}$ \\
\hline & $\mathrm{C}_{2}^{2}(10)$ & $\begin{array}{l}\mathrm{N} 1-\mathrm{H} 4 \cdots \mathrm{O} 3_{\text {carboxyl }} \mathrm{C} 16-\mathrm{H} 16 \cdots \mathbf{O 1}_{\text {carbonylFMOC }} \\
\mathrm{C} 14-\mathrm{H} 15 \cdots \mathbf{O}_{\text {carbonylFMOC \& }} \mathrm{C} 16-\mathrm{H} 16 \cdots \mathbf{O 1}_{\text {carbonylFMOC }} \\
\mathrm{C} 14-\mathrm{H} 14 \cdots \mathrm{O} 5_{\&} \mathrm{C} 16-\mathrm{H} 16 \cdots \mathbf{O 1}_{\text {carbonylFMO }}\end{array}$ \\
\hline & $\mathrm{C}_{2}^{2}(11)$ & $\begin{array}{l}\mathrm{O} 3_{\text {carboxyl }}-\mathrm{H} 1 \cdots \mathrm{O} 4_{\text {carbonyl \& }} \mathrm{C} 16-\mathrm{H} 16 \cdots \mathbf{O 1} \text { carbonylFMoc } \\
\mathrm{O} 5_{\text {hydroxyl }}-\mathrm{H} 2 \cdots \mathbf{O} 2_{\text {carbonylFMOC \& }} \mathrm{C} 16-\mathrm{H} 16 \cdots \mathbf{O} 1_{\text {carbonylFMOC }}\end{array}$ \\
\hline & $\mathrm{C}_{2}^{2}(12)$ & $\begin{array}{l}\mathrm{N} 1-\mathrm{H} 4 \cdots \mathrm{O}_{\text {carboxyl }} \mathrm{C} 14-\mathrm{H} 15 \cdots \mathbf{O}_{\text {carbonylFMOC }} \\
\left(\mathrm{C} 14-\mathrm{H} 15 \cdots \mathbf{O} 2_{\text {carbonylFMOC }}\right)_{2}\end{array}$ \\
\hline & $\mathrm{C}_{2}^{2}(13)$ & $\begin{array}{l}\mathrm{N} 1-\mathrm{H} 4 \cdots \mathrm{O} 3_{\text {carboxyl \& }} \mathrm{O} 3_{\text {carboxyl }}-\mathrm{H} 1 \cdots \mathrm{O} 4_{\text {carbonyl }} \\
\mathrm{N} 1-\mathrm{H} 4 \cdots{ }^{2} 3_{\text {carboxyl \& }} \mathrm{O}-\mathrm{H} 2 \cdots \mathbf{O} 2_{\text {carbonylFMOC }}\end{array}$ \\
\hline & $\mathrm{C}_{2}^{2}(14)$ & $\mathrm{N} 1-\mathrm{H} 4 \cdots \mathrm{O} 3_{\text {carboxyl \& }} \mathrm{C} 14-\mathrm{H} 14 \cdots \mathrm{O} 5$ \\
\hline & $\mathrm{C}_{2}^{2}(15)$ & 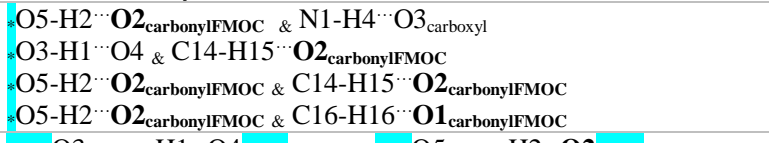 \\
\hline & $\mathrm{C}_{2}^{2}(16)$ & ${ }^{*}(\mathrm{OH}) 3_{\text {carboxyl }}-\mathrm{H} 1 \cdots \mathrm{O} 4_{(\mathrm{C}=\mathrm{O}) \text { carbonyl \& (OH) }} \mathrm{O} 5_{\text {hydroxyl }} \mathrm{H} 2 \cdots \mathbf{O} 2_{(\mathrm{C}=0) \text { carbonylFMOC }}$ \\
\hline
\end{tabular}




\begin{tabular}{|c|c|c|}
\hline & & $\begin{array}{l}{ }_{(\mathrm{NH})} \mathrm{N} 1-\mathrm{H} 4 \cdots \mathrm{O} 3_{(\mathrm{OH}) \text { carboxyl } \&(\mathrm{CH} 2)} \mathrm{C} 14-\mathrm{H} 14 \cdots \mathrm{O} 5_{(\mathrm{OH})} \\
{ }^{*(\mathrm{CH} 2)} \mathrm{C} 14-\mathrm{H} 15 \cdots \mathrm{O} 2_{(\mathrm{C}=\mathrm{O})} \&(\mathrm{CH} 2) \mathrm{C} 14-\mathrm{H} 14 \cdots \mathrm{O} 5_{(\mathrm{OH})} \\
{ }_{(\mathrm{CH} 2)} \mathrm{C} 14-\mathrm{H} 14 \cdots \mathrm{O} 5_{(\mathrm{OH})} \& \mathrm{C} 16-\mathrm{H} 16 \cdots \mathbf{O 1}_{\text {carbonylFMOC }}\end{array}$ \\
\hline & $\mathrm{C}_{2}^{2}(17)$ & $(\mathrm{OH}){ } 3_{\text {carboxyl }}-\mathrm{H} 1 \cdots \mathrm{O} 4_{(\mathrm{C}=\mathrm{O})}$ carbonyl \& $(\mathrm{CH} 2) \mathrm{C} 14-\mathrm{H} 14 \cdots \mathrm{O} 5_{(\mathrm{OH})}$ \\
\hline & $\mathrm{R}_{2}^{2}(7)$ & $\begin{array}{l}{ }_{(\mathrm{OH})} \mathrm{O} 3_{\text {carboxyl }}-\mathrm{H} 1 \cdots \mathrm{O} 4_{(\mathrm{C}=\mathrm{O}) \text { carbonyl } \&(\mathrm{NH})} \mathrm{N} 1-\mathrm{H} 4 \cdots \mathrm{O} 3_{(\mathrm{OH}) \text { carboxyl }} \\
{ }_{(\mathrm{OH})} \mathrm{O} 5-\mathrm{H} 2 \cdots 2_{(\mathrm{C}=\mathrm{O}) \text { carbonylFMOC } \&(\mathrm{CH} 2)} \mathrm{C} 14-\mathrm{H} 14 \cdots \mathrm{O} 5_{(\mathrm{OH})}\end{array}$ \\
\hline & $\mathrm{R}_{3}^{3}(12)$ & $\mathrm{N} 1-\mathrm{H} 4 \cdots \mathrm{O} 3_{\text {carboxyl \& }} \mathrm{C} 14-\mathrm{H} 15 \cdots \mathbf{O} 2_{\text {carbonylFMOC }}$ \\
\hline & $\mathrm{R}_{3}^{3}(15)$ & $\left(\mathrm{O} 3_{\text {carboxyl }}-\mathrm{H} 1 \cdots \mathrm{O} 4_{\text {carbonyl }}\right)_{2 \&} \mathrm{C} 16-\mathrm{H} 16 \cdots \mathbf{O} 1_{\text {carbonylFMOC }}$ \\
\hline & $\mathrm{R}_{3}^{3}(11)$ & {$\left[\mathrm{O} 3_{\text {carboxyl }}-\mathrm{H} 1 \cdots \mathrm{O} 4_{\text {carbonyl }}\right]_{2 \&} \mathrm{~N} 1-\mathrm{H} 4 \cdots \mathrm{O} 3_{\text {carboxyl }}$} \\
\hline & $\mathrm{R}_{3}^{3}(19)$ & $\mathrm{O} 3_{\text {carboxyl }}-\mathrm{H} 1 \cdots \mathrm{O} 4_{\text {carbony \& }} \mathrm{C} 14-\mathrm{H} 15 \cdots \mathbf{O} 2_{\text {carbonylFMOC }}$ \\
\hline & $\mathrm{R}_{4}^{4}(16)$ & {$\left[\mathrm{O}_{3 \text { carboxyl }}-\mathrm{H} 1 \cdots \mathrm{O} 4_{\text {carbonyl }}\right]_{2} \&\left[\mathrm{~N} 1-\mathrm{H} 4 \cdots \mathrm{O} 3_{\text {carboxyl }}\right]_{2}$} \\
\hline & $\mathrm{R}_{2}^{2}(8)$ & 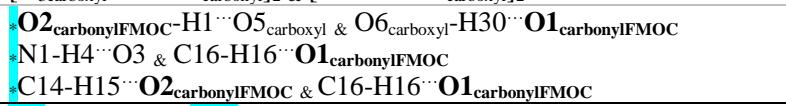 \\
\hline INEJEQ & $\mathrm{C}(4)$ & $(\mathrm{NH}) \mathrm{N} 1-\mathrm{H} 22 \cdots \mathbf{O 5}_{(\mathrm{C}=\mathrm{O}) \text { carbonylFMOC }}$ \\
\hline & $\mathrm{C}(6)$ & ${ }_{(\mathrm{CH} 3)} \mathrm{C} 13-\mathrm{H} 13 \cdots \mathrm{O} 3_{(\mathrm{C}=\mathrm{O})}$ \\
\hline & $\mathrm{C}(8)$ & $(\mathrm{CHcycl}) \mathrm{C} 1-\mathrm{H} 1 \cdots \mathbf{O 5}_{(\mathrm{C}=\mathbf{0}) \text { carbonylFMOC }}$ \\
\hline & $\mathbf{C}(12)$ & ${ }_{(\mathrm{CH} 3)} \mathrm{C} 15-\mathrm{H} 20 \cdots \mathrm{O} 2_{(\mathrm{C}-\mathrm{O}-\mathrm{C})}$ \\
\hline Level 2 & $\mathrm{C}_{2}^{2}(12)$ & $\mathrm{N} 1-\mathrm{H} 22 \cdots \mathbf{O 5}_{\text {carbonylFMOC \& }} \mathrm{C} 1-\mathrm{H} 1 \cdots \mathbf{O} 5_{\text {carbonylFMOC }}$ \\
\hline & $\mathrm{C}_{2}^{2}(14)$ & $\begin{array}{l}{ }_{(\mathrm{NH})} \mathrm{N} 1-\mathrm{H} 22 \cdots \mathbf{O 5}_{(\mathrm{C}=\mathrm{O})} \text { carbonylFMOC \& }{ }_{(\mathrm{CH} 3)} \mathrm{C} 13-\mathrm{H} 13 \cdots \mathrm{O} 3_{(\mathrm{C}=\mathrm{O})} \\
{ }_{(\mathrm{CH} y \mathrm{C})} \mathrm{C} 1-\mathrm{H} 1 \cdots \mathbf{O 5}_{(\mathrm{C}=\mathrm{O})} \text { carbonylFMOC } \&{ }_{(\mathrm{CH} 3)} \mathrm{C} 15-\mathrm{H} 20 \cdots \mathrm{O} 2_{(\mathrm{C}-\mathrm{O}-\mathrm{C})} \\
{ }_{(\mathrm{CH} 3)} \mathrm{C} 13-\mathrm{H} 13 \cdots \mathrm{O} 3_{(\mathrm{C}=\mathrm{O})} \&(\mathrm{CH} 3) \mathrm{C} 15-\mathrm{H} 20 \cdots \mathrm{O} 2_{(\mathrm{C}-\mathrm{O}-\mathrm{C})}\end{array}$ \\
\hline & $\mathrm{C}_{2}^{2}(16)$ & $(\mathrm{CHcycl}) \mathrm{C} 1-\mathrm{H} 1 \cdots \mathbf{O 5}_{(\mathrm{C}=\mathrm{O})}$ carbonylFMOC \& $(\mathrm{CH} 3) \mathrm{C} 13-\mathrm{H} 13 \cdots \mathrm{O} 3_{(\mathrm{C}=\mathrm{O})}$ \\
\hline & $\mathrm{C}_{2}^{2}(18)$ & $\begin{array}{l}{ }_{(\mathrm{NH})} \mathrm{N} 1-\mathrm{H} 22 \cdots \mathbf{O 5}_{(\mathrm{C}=\mathrm{O}) \text { carbonylFMoc } \&{ }_{(\mathrm{CH} 3)} \mathrm{C} 15-\mathrm{H} 20 \cdots \mathrm{O} 2_{(\mathrm{C}-\mathrm{O}-\mathrm{C})}} \mathrm{C} 13-\mathrm{H} 13 \cdots \mathrm{O} 3_{(\mathrm{C}=\mathrm{O})} \&{ }_{(\mathrm{CH} 3)} \mathrm{C} 15-\mathrm{H} 20 \cdots \mathrm{O} 2_{(\mathrm{C}-\mathrm{O}-\mathrm{C})}\end{array}$ \\
\hline & $\mathrm{C}_{2}^{2}(\mathbf{2 0})$ & ${ }_{(\mathrm{CHcycl})} \mathrm{C} 1-\mathrm{H} 1 \cdots \mathrm{O5}_{(\mathrm{C}=\mathrm{O})}$ carbonylFMOC \& $(\mathrm{CH} 3) \mathrm{C} 15-\mathrm{H} 20 \cdots \mathrm{O} 2_{(\mathrm{C}-\mathrm{O}-\mathrm{C})}$ \\
\hline & $\mathrm{R}_{2}^{1}(8)$ & 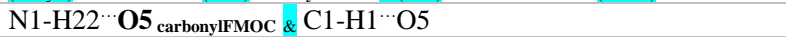 \\
\hline & $\mathbf{R}_{2}^{2}(14)$ & 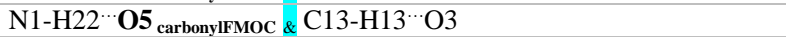 \\
\hline & $\mathbf{R}_{2}^{2}(16)$ & 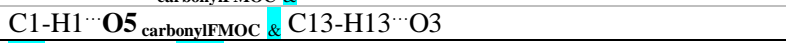 \\
\hline OGOGIA & $\mathrm{C}(4)$ & $(\mathrm{NH}) \mathrm{N} 1-\mathrm{H} 1 \cdots \mathbf{O} 2_{(\mathrm{C}=\mathrm{O}) \text { carbonylFMOC }}$ \\
\hline & $\mathrm{C}(11)$ & $(\mathrm{CH} 2) \mathrm{C} 26-\mathrm{H} 24 \cdots \mathrm{O} 3_{(\mathrm{C}=\mathrm{O})}$ \\
\hline & $\mathrm{C}(12)$ & ${ }_{(\mathrm{CH} 2)} \mathrm{C} 26-\mathrm{H} 23 \cdots \mathbf{O 1}$ (C-O-C)FMOC \\
\hline & $\mathrm{C}(15)$ & $\mathrm{C} 18-\mathrm{H} 15 \cdots \mathrm{N} 4$ \\
\hline Level 2 & $\mathrm{C}_{2}^{2}(10)$ & $\mathrm{C} 18-\mathrm{H} 15 \cdots \mathrm{N} 4$ \& $\mathrm{C} 26-\mathrm{H} 24 \cdots \mathrm{O} 3$ \\
\hline & $\mathrm{C}_{2}^{2}(13)$ & ${ }_{(\mathrm{CH} 3)} \mathrm{C} 18-\mathrm{H} 15 \cdots \mathrm{N} 4_{(\mathrm{NH})} \&(\mathrm{CH} 2) \mathrm{C} 26-\mathrm{H} 23 \cdots \mathbf{O 1}_{(\mathrm{C}-\mathrm{O}-\mathrm{C}) \mathrm{FMOC}}$ \\
\hline & $\mathrm{C}_{2}^{2}(14)$ & $\mathrm{N} 1-\mathrm{H} 1 \cdots \mathbf{O} 2$ carbonylFMOC \& $\mathrm{C} 26-\mathrm{H} 23 \cdots \mathbf{O 1}_{\text {FMOC }}$ \\
\hline & $\mathrm{C}_{2}^{2}(16)$ & $\mathrm{N} 1-\mathrm{H} 1 \cdots \mathbf{O} 2_{\text {carbonylFMOC \& }} \mathrm{C} 26-\mathrm{H} 23 \cdots \mathbf{O 1}_{\text {FMOC }}$ \\
\hline & $\mathrm{C}_{2}^{2}(17)$ & ${ }_{(\mathrm{NH})} \mathrm{N} 1-\mathrm{H} 1 \cdots \mathbf{O} 2_{(\mathrm{C}=\mathrm{O}) \text { carbonylFMOC \& }(\mathrm{CH} 2)} \mathrm{C} 26-\mathrm{H} 24 \cdots \mathrm{O} 3_{(\mathrm{C}=\mathrm{O})}$ \\
\hline & $\mathrm{R}_{2}^{2}(9)$ & $\mathrm{C} 26-\mathrm{H} 23 \cdots \mathbf{O 1}_{\mathrm{FMOC} \&} \mathrm{C} 26-\mathrm{H} 24 \cdots \mathrm{O} 3$ \\
\hline OGIYAG & $S(6)$ & ${ }_{(\mathrm{CH} 2)} \mathrm{C} 18-\mathrm{H} 16 \cdots \mathbf{O} 2$ carbonylFMOC \\
\hline & $\mathrm{C}(5)$ & 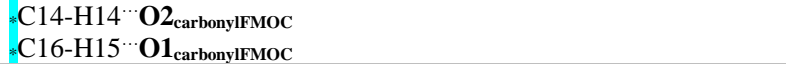 \\
\hline & $\mathrm{R}_{2}^{2}(20)$ & {$\left[\mathrm{O} 5_{\text {hydroxy }}-\mathrm{H} 2 \cdots \mathrm{O} 3_{\text {carboxy }}\right]_{2}$} \\
\hline & $\mathrm{D}(2)$ & $\begin{array}{l}\mathrm{O} 4_{\text {carboxyl }}-\mathrm{H} 1 \cdots \mathrm{O}_{\text {water }} \\
\mathrm{O} 6-\mathrm{H} 22 \cdots \mathrm{O} 5_{\text {hydroxy }} \\
\mathrm{O} 6_{\text {water }}-\mathrm{H} 23 \cdots \mathbf{O} 2_{\text {carbonylFMoc }}\end{array}$ \\
\hline level 2 & $\mathrm{C}_{2}^{2}(9)$ & $\mathrm{O} 4-\mathrm{H} 1 \cdots \mathrm{O} 6_{\&} \mathrm{O} 6-\mathrm{H} 23 \cdots \mathbf{O} 2_{\text {carbonylFMoc }}$ \\
\hline & $\mathrm{C}_{2}^{2}(10)$ & ${ }_{(\mathrm{CH})} \mathrm{C} 16-\mathrm{H} 15 \cdots \mathbf{O 1}_{(\mathrm{C}-\mathrm{O}-\mathrm{C}) \text { carbonylFMOC \& }(\mathrm{CH} 2)} \mathrm{C} 14-\mathrm{H} 14 \cdots \mathbf{O 2}_{(\mathrm{C}=\mathrm{O}) \text { carbonylFMOC }}$ \\
\hline & $\mathrm{C}_{2}^{2}(12)$ & $(\mathrm{OH}) \mathrm{O} 4-\mathrm{H} 1 \cdots 6_{(\mathrm{H} 2 \mathrm{O})} \&(\mathrm{H} 2 \mathrm{O}) \mathrm{O} 6-\mathrm{H} 22 \cdots \mathrm{O} 5_{(\mathrm{OH})}$ \\
\hline & $\mathrm{C}_{2}^{2}(13)$ & ${ }_{(\mathrm{H} 2 \mathrm{O})} \mathrm{O} 6-\mathrm{H} 23 \cdots \mathbf{O} 2_{(\mathrm{C}=\mathrm{O}) \text { carbonylFMOC \& (H2O) }} \mathrm{O} 6-\mathrm{H} 22 \cdots \mathrm{O} 5_{(\mathrm{OH})}$ \\
\hline & $\mathrm{C}_{2}^{2}(15)$ & $\mathrm{O} 5-\mathrm{H} 2 \cdots \mathrm{O} 3_{\&} \mathrm{C} 16-\mathrm{H} 15 \cdots \mathbf{O 1}_{\text {carbonylFMOC }}$ \\
\hline & $\mathrm{C}_{2}^{2}(19)$ & ${ }_{(\mathrm{OH})} \mathrm{O} 5-\mathrm{H} 2 \cdots \mathrm{O} 3_{(\mathrm{C}=\mathrm{O})} \&(\mathrm{CH} 2) \mathrm{C} 14-\mathrm{H} 14 \cdots \mathbf{O 2}_{(\mathrm{C}=\mathrm{O}) \text { carbonylFMOC }}$ \\
\hline & $\mathrm{R}_{2}^{2}(8)$ & 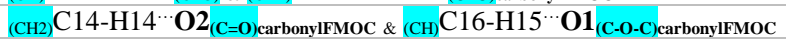 \\
\hline & $\mathrm{D}_{3}^{2}(8)$ & $\mathrm{O} 6-\mathrm{H} 23 \cdots \mathbf{O} 2_{\text {carbonylFMOC \& }} \mathrm{C} 14-\mathrm{H} 14 \cdots \mathbf{O} 2_{\text {carbonylFMOC }}$ \\
\hline & $\mathrm{D}_{3}^{3}(10)$ & O6-H23 $\cdots 22_{\text {carbonylFMOC \& }}$ C16-H15 $\cdots 1_{\text {carbonylFMOC }}$ \\
\hline & $\mathrm{D}_{3}^{3}(13)$ & $\mathrm{O} 5-\mathrm{H} 2 \cdots \mathrm{O} 3 \&[\mathrm{O} 6-\mathrm{H} 22 \cdots \mathrm{O} 5]_{2}$ \\
\hline & $\mathrm{D}_{3}^{3}(14)$ & {$[\mathrm{O} 4-\mathrm{H} 1 \cdots \mathrm{O} 6]_{2} \& \mathrm{C} 16-\mathrm{H} 15 \cdots \mathbf{O 1}_{\text {carbonylFMoc }}$} \\
\hline & $\mathrm{D}_{3}^{3}(17)$ & {$[\mathrm{O} 4-\mathrm{H} 1 \cdots \mathrm{O} 6]_{2} \& \mathrm{O} 5-\mathrm{H} 2 \cdots \mathrm{O} 3$} \\
\hline & $\mathrm{D}_{3}^{3}(18)$ & {$[\mathrm{O} 4-\mathrm{H} 1 \cdots \mathrm{O} 6]_{2} \& \mathrm{C} 14-\mathrm{H} 14 \cdots \mathbf{O} 2_{\text {carbonylFMoc }}$} \\
\hline & $\mathrm{D}_{3}^{3}(19)$ & $\mathrm{O} 5-\mathrm{H} 2 \cdots \mathrm{O} 3_{\&} \mathrm{O} 6-\mathrm{H} 23 \cdots \mathbf{O} 2_{\text {carbonylFMOc }}$ \\
\hline & $\mathrm{D}_{3}^{3}(20)$ & $(\mathrm{O} 5-\mathrm{H} 22 \cdots \mathrm{O} 6)_{2 \&} \mathrm{C} 16-\mathrm{H} 15 \cdots \mathbf{O 1}_{\text {carbonylFMoc }}$ \\
\hline & & group $\boldsymbol{B}$ \\
\hline OGIXOT & $\mathrm{C}(4)$ & $\begin{array}{l}{ }_{(\mathrm{OH})} \mathrm{O} 3-\mathrm{H} 1_{\text {carboxyl }} \cdots \mathrm{O} 4_{(\mathrm{C}=\mathrm{O}) \text { carbonyl }} \\
{ }_{(\mathrm{NH})} \mathrm{N} 1-\mathrm{H} 8 \cdots \mathrm{O} 2_{(\mathrm{C}=\mathrm{O}) \text { carbonylFMoc }}\end{array}$ \\
\hline & $C(10)$ & ${ }_{(\mathrm{CH} \text { cycl) }} \mathrm{C} 21-\mathrm{H} 16 \cdots \mathrm{O} 1_{(\mathrm{C}-\mathrm{O}-\mathrm{C})}$ \\
\hline & $C(14)$ & 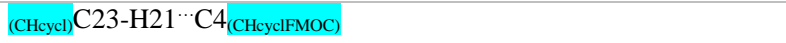 \\
\hline & $\mathrm{C}_{2}^{2}(12)$ & $\begin{array}{l}\mathrm{O} 3_{\text {carboxyl }}-\mathrm{H} 1{ }^{\cdots} \mathrm{O} 4_{\text {carbonyl }} \& \mathrm{~N} 1-\mathrm{H} 8 \cdots \mathrm{O} 2_{\text {carbonylFMOC }} \\
\mathrm{N} 1-\mathrm{H} 8{ }^{\cdots} \mathrm{O} 2_{\text {carbonylFMOC \& }} \mathrm{C} 21-\mathrm{H} 16 \cdots \mathrm{O} 1\end{array}$ \\
\hline & $\mathrm{C}_{2}^{2}(14)$ & $* \mathrm{~N} 1-\mathrm{H} 8 \cdots \mathrm{O} 2$ carbonylFMOc \& $\mathrm{C} 21-\mathrm{H} 16 \cdots \mathrm{O} 1$ \\
\hline Lev. 2 & $\mathrm{C}_{2}^{2}(16)$ & ${ }_{(\mathrm{OH})} \mathrm{O} 3-\mathrm{H} 1 \cdots \mathrm{O} 4_{(\mathrm{C}=\mathrm{O})} \&(\mathrm{CHcycl}) \mathrm{C} 21-\mathrm{H} 16 \cdots \mathrm{O} 1_{(\mathrm{C}-\mathrm{O}-\mathrm{C})}$ \\
\hline & $\mathrm{R}_{3}^{3}(16)$ & {$\left[\mathrm{O} 3_{\text {carboxyl }}-\mathrm{H} 1 \cdots \mathrm{O} 4_{\text {carbonyl }}\right]_{2 \&} \mathrm{~N} 1-\mathrm{H} 8 \cdots \mathrm{O} 2_{\text {carbonylFMOC }}$} \\
\hline EKEWUM & & \\
\hline & $\mathrm{C}(5)$ & $\begin{array}{l}{ }_{(\mathrm{CH})} \mathrm{C} 7-\mathrm{H} 7 \cdots \mathrm{O} 4_{(\mathrm{C}-\mathrm{O}-\mathrm{C})} \\
{ }_{(\mathrm{CH} 2)} \mathrm{C} 11-\mathrm{H} 13 \cdots \mathrm{O} 3_{(\mathrm{C}=\mathrm{O})} \\
{ }_{(\mathrm{NH})} \mathrm{N} 2-\mathrm{H} 11 \cdots \mathrm{O} 2_{(\mathrm{C}-\mathrm{O}-\mathrm{C})}\end{array}$ \\
\hline & $\mathrm{C}(8)$ & ${ }_{(\mathrm{CHcycl})} \mathrm{C} 24-\mathrm{H} 22 \cdots \mathrm{O} 3_{(\mathrm{C}=\mathrm{O})}$ \\
\hline & $\mathrm{C}(12)$ & ${ }_{(\mathrm{CHcycl})} \mathrm{C} 21-\mathrm{H} 19 \cdots \mathrm{O} 1_{(\mathrm{C}=\mathrm{O})}$ \\
\hline & $\mathrm{S}(11)$ & ${ }_{(\mathrm{CHcycl})} \mathrm{C} 18-\mathrm{H} 18 \cdots \mathrm{O} 1_{(\mathrm{C}=\mathrm{O})}$ \\
\hline
\end{tabular}




\begin{tabular}{|c|c|c|}
\hline \multirow[t]{12}{*}{ Level 2} & $\mathrm{C}_{2}^{2}(10)$ & $\begin{array}{l}{ }_{(\mathrm{NH})} \mathrm{N} 2-\mathrm{H} 11 \cdots \mathrm{O} 2_{(\mathrm{C}=\mathrm{O})} \&(\mathrm{CH}) \mathrm{C} 7-\mathrm{H} 7 \cdots \mathrm{O} 4_{(\mathrm{C}-\mathrm{O}-\mathrm{C})} \\
{ }_{(\mathrm{CH})} \mathrm{C} 7-\mathrm{H} 7 \cdots \mathrm{O} 4_{(\mathrm{C}-\mathrm{O}-\mathrm{C})} \&(\mathrm{CH} 2) \\
\mathrm{C} 11-\mathrm{H} 13 \cdots \mathrm{O} 3_{(\mathrm{C}=\mathrm{O})}\end{array}$ \\
\hline & $\mathrm{C}_{2}^{2}(11)$ & ${ }_{(\mathrm{CH})} \mathrm{C} 7-\mathrm{H} 7 \cdots \mathrm{O} 4_{(\mathrm{C}-\mathrm{O}-\mathrm{C})} \&(\mathrm{CHcycl}) \mathrm{C} 21-\mathrm{H} 19 \cdots \mathrm{O} 1_{(\mathrm{C}=\mathrm{O})}$ \\
\hline & $\mathrm{C}_{2}^{2}(12)$ & 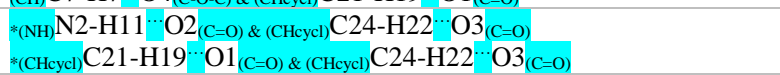 \\
\hline & $\mathrm{C}_{2}^{2}(13)$ & 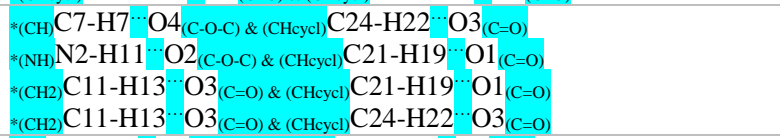 \\
\hline & $\mathrm{C}_{2}^{2}(15)$ & ${ }_{(\mathrm{NH})} \mathrm{N} 2-\mathrm{H} 11^{\cdots} \mathrm{O} 2_{(\mathrm{C}-\mathrm{O}-\mathrm{C})} \&{ }_{(\mathrm{CHcycl})} \mathrm{C} 24-\mathrm{H} 22 \cdots \mathrm{O} 3_{(\mathrm{C}=\mathrm{O})}$ \\
\hline & $\mathrm{C}_{2}^{2}(17)$ & 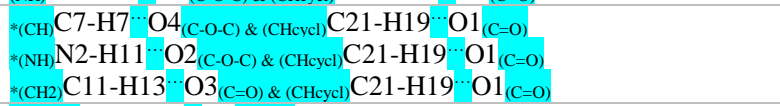 \\
\hline & $\mathrm{C}_{2}^{2}(20)$ & {$\left[(\mathrm{CHcycl}) \mathrm{C} 21-\mathrm{H} 19 \cdots \mathrm{O} 1_{(\mathrm{C}=\mathrm{O})]_{2}}\right.$} \\
\hline & $\mathrm{R}_{2}^{1}(7)$ & ${ }_{(\mathrm{CH} 2)} \mathrm{C} 11-\mathrm{H} 13 \cdots \mathrm{O} 3_{(\mathrm{C}=\mathrm{O})} \&(\mathrm{CHcycl}) \mathrm{C} 24-\mathrm{H} 22 \cdots \mathrm{O} 3_{(\mathrm{C}=\mathrm{O})}$ \\
\hline & $\mathrm{R}_{2}^{2}(8)$ & $\begin{array}{l}*_{(\mathrm{NH})} \mathrm{N} 2-\mathrm{H} 11 \cdots \mathrm{O} 2_{(\mathrm{C}-\mathrm{O}-\mathrm{C})} \&{ }_{(\mathrm{CH})} \mathrm{C} 7-\mathrm{H} 7 \cdots \mathrm{O} 4_{(\mathrm{C}-\mathrm{O}-\mathrm{C})} \\
{ }_{(\mathrm{CH})} \mathrm{C} 7-\mathrm{H} 7 \cdots \mathrm{O} 4_{(\mathrm{C}-\mathrm{O}-\mathrm{C})} \&(\mathrm{CH}) \mathrm{C} 11-\mathrm{H} 13 \cdots \mathrm{O} 3_{(\mathrm{C}=\mathrm{O})}\end{array}$ \\
\hline & $\mathrm{R}_{2}^{2}(11)$ & ${ }_{(\mathrm{CH})} \mathrm{C} 7-\mathrm{H} 7 \cdots \mathrm{O} 4_{(\mathrm{C}-\mathrm{O}-\mathrm{C})} \&(\mathrm{CHcycl}) \mathrm{C} 24-\mathrm{H} 22 \cdots \mathrm{O} 3_{(\mathrm{C}=\mathrm{O})}$ \\
\hline & $\mathrm{R}_{2}^{2}(12)$ & ${ }_{(\mathrm{NH})} \mathrm{N} 2-\mathrm{H} 11 \cdots \mathrm{O} 2_{(\mathrm{C}-\mathrm{O}-\mathrm{C})} \&(\mathrm{CH} 2) \mathrm{C} 11-\mathrm{H} 13 \cdots \mathrm{O} 3_{(\mathrm{C}=\mathrm{O})}$ \\
\hline & $\mathrm{R}_{2}^{2}(15)$ & ${ }_{(\mathrm{NH})} \mathrm{N} 2-\mathrm{H} 11 \cdots \mathrm{O} 2_{(\mathrm{C}-\mathrm{O}-\mathrm{C})} \&(\mathrm{CHcycl}) \mathrm{C} 24-\mathrm{H} 22 \cdots \mathrm{O} 3_{(\mathrm{C}=\mathrm{O})}$ \\
\hline \multicolumn{3}{|r|}{ 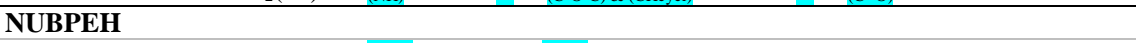 } \\
\hline & $\mathrm{C}(4)$ & $\begin{array}{l}{ }_{(\mathrm{NH})} \mathrm{N} 1-\mathrm{H} 2 \cdots \mathrm{O} 3_{(\mathrm{C}=\mathrm{O})} \\
{ }_{(\mathrm{NH})} \mathrm{N} 2-\mathrm{H} 3 \cdots \mathrm{O} 44_{(\mathrm{C}=\mathrm{O})} \\
{ }_{(\mathrm{NH})} \mathrm{N} 3-\mathrm{H} 32 \cdots \mathrm{O} 13_{(\mathrm{C}=\mathrm{O})} \\
{ }_{(\mathrm{NH})} \mathrm{N} 1-\mathrm{H} 33 \cdots \mathrm{O} 12_{(\mathrm{C}=\mathrm{O})} \\
{ }^{*}{ }_{(\mathrm{CH})} \mathrm{C} 41-\mathrm{H} 41 \cdots \mathrm{O} 12_{(\mathrm{C}=\mathrm{O})} \\
{ }_{(\mathrm{CH})} \mathrm{C} 43-\mathrm{H} 42 \cdots \mathrm{O} 10_{(\mathrm{C}=\mathrm{O})}\end{array}$ \\
\hline & $\mathrm{C}(6)$ & ${ }_{(\mathrm{CH} 2)} \mathrm{C} 27-\mathrm{H} 24 \cdots \mathrm{O} 3_{(\mathrm{C}=\mathrm{O})}$ \\
\hline & $\mathrm{D}(2)$ & 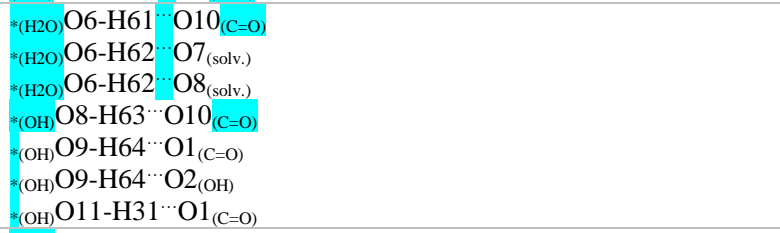 \\
\hline & $\mathrm{D}_{2}^{1}(3)$ & $(\mathrm{H} 2 \mathrm{O}) \mathrm{O} 6-\mathrm{H} 62 \cdots \mathrm{O} 7_{\text {(solv. })}$ \\
\hline & $\mathrm{D}_{2}^{2}(5)$ & {$\left[(\mathrm{H} 2 \mathrm{O}) \mathrm{O} 6-\mathrm{H} 62 \cdots \mathrm{O} 8_{\left.\left(\text {solv }_{*}\right)\right]_{2}}\right.$} \\
\hline & $\mathrm{D}_{2}^{2}(7)$ & ${ }_{(\mathrm{OH})} \mathrm{O} 8 \mathrm{~A}-\mathrm{H} 66 \cdots \mathrm{O} 10_{(\mathrm{C}=\mathrm{O})} \&(\mathrm{OH}) \mathrm{O} 8-\mathrm{H} 63 \cdots \mathrm{O} 10_{(\mathrm{C}=\mathrm{O})}$ \\
\hline \multirow[t]{27}{*}{ Level 2} & $\mathrm{C}(12)$ & ${ }_{(\mathrm{NH})} \mathrm{N} 2-\mathrm{H} 3 \cdots \mathrm{O} 4_{(\mathrm{C}=\mathrm{O})}$ \\
\hline & $\mathrm{C}_{2}^{2}(8)$ & ${ }_{(\mathrm{NH})} \mathrm{N} 4-\mathrm{H} 33 \cdots \mathrm{O} 12_{(\mathrm{C}=\mathrm{O})} \&(\mathrm{CH}) \mathrm{C} 41-\mathrm{H} 41 \cdots \mathrm{O} 12_{(\mathrm{C}=\mathrm{O})}$ \\
\hline & $\mathrm{C}_{2}^{2}(10)$ & $\begin{array}{l}*_{(\mathrm{NH})} \mathrm{N} 1-\mathrm{H} 2 \cdots \mathrm{O} 3_{(\mathrm{C}=\mathrm{O})} \&(\mathrm{CH} 2) \mathrm{C} 27-\mathrm{H} 24 \cdots \mathrm{O} 3_{(\mathrm{C}=\mathrm{O})} \\
{ }_{(\mathrm{NH})} \mathrm{N} 3-\mathrm{H} 32 \cdots \mathrm{O} 13_{(\mathrm{C}=\mathrm{O})} \&(\mathrm{CH}) \mathrm{C} 41-\mathrm{H} 41 \cdots \mathrm{O} 12_{(\mathrm{C}=\mathrm{O})} \\
*_{(\mathrm{NH})} \mathrm{N} 4-\mathrm{H} 33 \cdots \mathrm{O} 12_{(\mathrm{C}=\mathrm{O})} \&(\mathrm{CH}) \\
\mathrm{C} 43-\mathrm{H} 42 \cdots \mathrm{O} 10_{(\mathrm{C}=\mathrm{O})}\end{array}$ \\
\hline & $\mathrm{C}_{2}^{2}(12)$ & $\begin{array}{l}{ }_{(\mathrm{NH})} \mathrm{N} 3-\mathrm{H} 32 \cdots \mathrm{O} 13_{(\mathrm{C}=\mathrm{O})} \&(\mathrm{NH}) \mathrm{N} 4-\mathrm{H} 33 \cdots \mathrm{O} 12_{(\mathrm{C}=\mathrm{O})} \\
{ }_{(\mathrm{CH})} \mathrm{C} 41-\mathrm{H} 41^{\cdots} \mathrm{O} 12_{(\mathrm{C}=\mathrm{O})} \&(\mathrm{CH}) \mathrm{C} 43-\mathrm{H} 42 \cdots \mathrm{O} 10_{(\mathrm{C}=\mathrm{O})}\end{array}$ \\
\hline & $\mathrm{C}_{2}^{2}(14)$ & ${ }_{(\mathrm{NH})} \mathrm{N} 2-\mathrm{H} 3 \cdots \mathrm{O} 4_{(\mathrm{C}=\mathrm{O})} \&(\mathrm{CH} 2) \mathrm{C} 27-\mathrm{H} 24 \cdots \mathrm{O} 3_{(\mathrm{C}=\mathrm{O})}$ \\
\hline & $\mathrm{C}_{2}^{2}(16)$ & ${ }_{(\mathrm{NH})} \mathrm{N} 3-\mathrm{H} 32 \cdots \mathrm{O} 13_{(\mathrm{C}=\mathrm{O})} \&(\mathrm{CH}) \mathrm{C} 43-\mathrm{H} 42 \cdots \mathrm{O} 10_{(\mathrm{C}=\mathrm{O})}$ \\
\hline & $\mathrm{C}_{3}^{3}(10)$ & ${ }_{\text {(solv. })} \mathrm{O} 8-\mathrm{H} 63 \cdots \mathrm{O} 10_{(\mathrm{C}=\mathrm{O})]_{2} \&(\mathrm{CH})} \mathrm{C} 43-\mathrm{H} 42 \cdots \mathrm{O} 10_{(\mathrm{C}=\mathrm{O})}$ \\
\hline & $\mathrm{C}_{3}^{3}(16)$ & {$\left[(\mathrm{OH}) \mathrm{O} 8-\mathrm{H} 63 \cdots \mathrm{O} 10_{(\mathrm{C}=\mathrm{O})}\right]_{2} \&(\mathrm{NH}) \mathrm{N} 4-\mathrm{H} 33^{\cdots} \mathrm{O} 12_{(\mathrm{C}=\mathrm{O})}$} \\
\hline & $\mathrm{C}_{3}^{3}(18)$ & 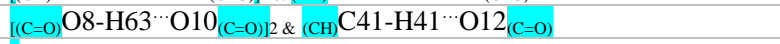 \\
\hline & $\mathrm{R}_{2}^{1}(6)$ & $\begin{array}{l}{ }_{(\mathrm{NH})} \mathrm{N} 1-\mathrm{H} 2 \cdots \mathrm{O} 3_{(\mathrm{C}=\mathrm{O})} \&(\mathrm{CH} 2) \mathrm{C} 27-\mathrm{H} 24 \cdots \mathrm{O} 3_{(\mathrm{C}=\mathrm{O})} \\
{ }_{(\mathrm{NH})} \mathrm{N} 4-\mathrm{H} 33 \cdots \mathrm{O} 12_{(\mathrm{C}=\mathrm{O})} \&(\mathrm{CH}) \mathrm{C} 41-\mathrm{H} 41 \cdots \mathrm{O} 12_{(\mathrm{C}=\mathrm{O})}\end{array}$ \\
\hline & $\mathrm{R}^{2}{ }_{1}(3)$ & ${ }_{(\mathrm{H} 2 \mathrm{O})} \mathrm{O} 6-\mathrm{H} 62 \cdots \mathrm{O} 7_{(\text {solv. })} \&(\mathrm{H} 2 \mathrm{O}) \mathrm{O} 6-\mathrm{H} 62 \cdots \mathrm{O} 8_{(\mathrm{C}=\mathrm{O})}$ \\
\hline & $\mathrm{R}_{1}^{2}(4)$ & ${ }_{(\mathrm{H} 2 \mathrm{O})} \mathrm{O} 9-\mathrm{H} 64 \cdots \mathrm{O} 1_{(\mathrm{C}=\mathrm{O})} \&(\mathrm{H} 2 \mathrm{O}) \mathrm{O} 9-\mathrm{H} 64 \cdots \mathrm{O} 2_{(\mathrm{OH})}$ \\
\hline & $\mathrm{R}_{2}^{2}(10)$ & $\begin{array}{l}{ }_{(\mathrm{NH})} \mathrm{N} 3-\mathrm{H} 32 \cdots \mathrm{O} 13_{(\mathrm{C}=\mathrm{O})} \&(\mathrm{CH}) \mathrm{C} 41-\mathrm{H} 41 \cdots \mathrm{O} 12_{(\mathrm{C}=\mathrm{O})} \\
\left.{ }^{*} \mathrm{NH}\right) \\
\mathrm{N} 4-\mathrm{H} 33 \cdots \mathrm{O} 12_{(\mathrm{C}=\mathrm{O})} \&(\mathrm{CH}) \mathrm{C} 43-\mathrm{H} 42 \cdots \mathrm{O} 10_{(\mathrm{C}=\mathrm{O})}\end{array}$ \\
\hline & $\mathrm{R}_{2}^{2}(12)$ & $\begin{array}{l}{ }_{(\mathrm{NH})} \mathrm{N} 2-\mathrm{H} 3 \cdots \mathrm{O} 4_{(\mathrm{C}=\mathrm{O})} \&(\mathrm{NH}) \mathrm{N} 1-\mathrm{H} 2 \cdots \mathrm{O} 3_{(\mathrm{C}=\mathrm{O})} \\
{ }_{(\mathrm{NH})} \mathrm{N} 3-\mathrm{H} 32 \cdots \mathrm{O} 13_{(\mathrm{C}=\mathrm{O})} \&(\mathrm{NH}) \mathrm{N} 4-\mathrm{H} 33^{\cdots} \mathrm{O} 12_{(\mathrm{C}=\mathrm{O})} \\
{ }_{(\mathrm{CH})} \mathrm{C} 41-\mathrm{H} 41 \cdots \mathrm{O} 12_{(\mathrm{C}=\mathrm{O})} \&(\mathrm{CH}) \\
\mathrm{C} 43-\mathrm{H} 42 \cdots \mathrm{O} 10_{(\mathrm{C}=\mathrm{O})}\end{array}$ \\
\hline & $\mathrm{R}_{2}^{2}(14)$ & ${ }_{(\mathrm{NH})} \mathrm{N} 2-\mathrm{H} 3 \cdots \mathrm{O} 4_{(\mathrm{C}=\mathrm{O})} \&(\mathrm{CH} 2) \mathrm{C} 27-\mathrm{H} 24 \cdots \mathrm{O} 3_{(\mathrm{C}=\mathrm{O})}$ \\
\hline & $\mathrm{R}_{2}^{2}(16)$ & ${ }_{(\mathrm{NH})} \mathrm{N} 3-\mathrm{H} 32 \cdots \mathrm{O} 13_{(\mathrm{C}=\mathrm{O})} \&(\mathrm{CH}) \mathrm{C} 43-\mathrm{H} 42 \cdots \mathrm{O} 10_{(\mathrm{C}=\mathrm{O})}$ \\
\hline & $\mathrm{R}_{6}^{4}(20)$ & {$\left[(\mathrm{OH}) \mathrm{O} 8-\mathrm{H} 63 \cdots \mathrm{O} 10_{(\mathrm{C}=\mathrm{O})] 2} \&(\mathrm{CH}) \mathrm{C} 43-\mathrm{H} 42 \cdots \mathrm{O} 10_{(\mathrm{C}=\mathrm{O})}\right.$} \\
\hline & $\mathrm{D}_{2}^{1}(3)$ & $\begin{array}{l}{ }_{(\mathrm{H} 2 \mathrm{O})} \mathrm{O} 6-\mathrm{H} 61 \cdots \mathrm{O} 10_{(\mathrm{C}=\mathrm{O})} \&(\mathrm{OH}) \\
{ }_{(\mathrm{H} 2 \mathrm{O})} \mathrm{O} 9-\mathrm{H} 64 \cdots \mathrm{O} 1_{(\mathrm{C}=\mathrm{O})} \&(\mathrm{OH}) \mathrm{O} 11-\mathrm{H} 31^{\cdots} \mathrm{O} 1_{(\mathrm{C}=\mathrm{O})}\end{array}$ \\
\hline & $\mathrm{D}_{2}^{2}(4)$ & ${ }_{(\mathrm{H} 2 \mathrm{O})} \mathrm{O} 6-\mathrm{H} 62 \cdots \mathrm{O} 8_{(\mathrm{C}=\mathrm{O})} \&(\mathrm{OH}) \mathrm{O} 8-\mathrm{H} 63 \cdots \mathrm{O} 10_{(\mathrm{C}=\mathrm{O})}$ \\
\hline & $\mathrm{D}_{2}^{2}(5)$ & $\begin{array}{l}{ }_{(\mathrm{H} 2 \mathrm{O})} \mathrm{O} 6-\mathrm{H} 61 \cdots \mathrm{O} 10_{(\mathrm{C}=\mathrm{O})} \&(\mathrm{H} 2 \mathrm{O}) \mathrm{O} 6-\mathrm{H} 62 \cdots \mathrm{O} 7_{(\mathrm{solv} .)} \\
{ }_{(\mathrm{H} 2 \mathrm{O})} \mathrm{O} 6-\mathrm{H} 61 \cdots \mathrm{O} 10_{(\mathrm{C}=\mathrm{O})} \&(\mathrm{H} 2 \mathrm{O}) \mathrm{O} 6-\mathrm{H} 62 \cdots \mathrm{O} 8_{(\mathrm{C}=\mathrm{O})} \\
{ }_{(\mathrm{H} 2 \mathrm{O})} \mathrm{O} 6-\mathrm{H} 62 \cdots \mathrm{O} 7_{(\mathrm{solv})} \&(\mathrm{OH}) \mathrm{O} 8-\mathrm{H} 63 \cdots \mathrm{O} 1_{(\mathrm{C}=\mathrm{O})} \\
{ }_{(\mathrm{H} 2 \mathrm{O})} \mathrm{O} 9-\mathrm{H} 64 \cdots \mathrm{O} 2_{(\mathrm{OH})} \&(\mathrm{OH}) \mathrm{O} 11-\mathrm{H} 31 \cdots \mathrm{O} 1_{(\mathrm{C}=\mathrm{O})}\end{array}$ \\
\hline & $\mathrm{D}_{2}^{2}(6)$ & $\begin{array}{l}{ }_{(\mathrm{H} 2 \mathrm{O})} \mathrm{O} 6-\mathrm{H} 61 \cdots \mathrm{O} 10_{(\mathrm{C}=\mathrm{O})} \&(\mathrm{OH}) \mathrm{O} 11-\mathrm{H} 31 \cdots \mathrm{O} 1_{(\mathrm{C}=\mathrm{O})]} \\
\text { (solv. })^{\mathrm{O}} \mathrm{O} 8 \mathrm{~A}-\mathrm{H} 66 \cdots \mathrm{O} 10_{(\mathrm{C}=\mathrm{O})} \&(\mathrm{H} 2 \mathrm{O}) \\
{ }_{\text {(solv. })} \mathrm{O} 6-\mathrm{H} 62 \cdots \mathrm{O} 28_{(\mathrm{C}=\mathrm{O})}\end{array}$ \\
\hline & $\mathrm{D}_{3}^{2}(6)$ & $(\mathrm{H} 2 \mathrm{O}) \mathrm{O} 6-\mathrm{H} 61 \cdots \mathrm{O} 10_{(\mathrm{C}=\mathrm{O})} \&(\mathrm{H} 2 \mathrm{O}) \mathrm{O} 6-\mathrm{H} 62 \cdots \mathrm{O} 7_{(\text {solv. }}$ \\
\hline & $\mathrm{D}_{3}^{2}(7)$ & {$\left[(\mathrm{H} 2 \mathrm{O}) \mathrm{O} 6-\mathrm{H} 61 \cdots \mathrm{O} 10_{(\mathrm{C}=\mathrm{O})] 2} \&(\mathrm{CH}) \mathrm{C} 43-\mathrm{H} 42 \cdots \mathrm{O} 10_{(\mathrm{C}=\mathrm{O})}\right.$} \\
\hline & $\mathrm{D}_{3}^{2}(8)$ & ${ }_{(\mathrm{OH})} \mathrm{O} 8-\mathrm{H} 63 \cdots \mathrm{O} 10_{(\mathrm{C}=\mathrm{O})} \&(\mathrm{OH}) \mathrm{O} 8-\mathrm{H} 66 \cdots \mathrm{O} 10_{(\mathrm{C}=\mathrm{O})} \&(\mathrm{H} 2 \mathrm{O}) \mathrm{O} 6-\mathrm{H} 61 \cdots \mathrm{O} 10_{(\mathrm{C}=\mathrm{O})}$ \\
\hline & $\mathrm{D}_{3}^{3}(8)$ & ${ }_{(\mathrm{H} 2 \mathrm{O})} \mathrm{O} 6-\mathrm{H} 61 \cdots \mathrm{O} 10_{(\mathrm{C}=\mathrm{O})} \&(\mathrm{H} 2 \mathrm{O}) \mathrm{O} 6-\mathrm{H} 62 \cdots \mathrm{O} 8_{(\mathrm{C}=\mathrm{O})}$ \\
\hline & $\mathrm{D}_{3}^{3}(11)$ & $\begin{array}{l}{ }_{(\mathrm{OH})} \mathrm{O} 8-\mathrm{H} 63 \cdots \mathrm{O} 10_{(\mathrm{C}=\mathrm{O})} \&(\mathrm{OH}) \mathrm{O} 8 \mathrm{~A}-\mathrm{H} 66 \cdots \mathrm{O} 10_{(\mathrm{C}=\mathrm{O})} \\
{ }_{[(\mathrm{OH})} \mathrm{O} 11-\mathrm{H} 31 \cdots \mathrm{O} 1_{(\mathrm{C}=\mathrm{O})] 2} \&(\mathrm{CH}) \\
\mathrm{C} 43-\mathrm{H} 42 \cdots \mathrm{O} 1_{(\mathrm{C}=\mathrm{O})}\end{array}$ \\
\hline & $\mathrm{D}_{3}^{3}(13)$ & $\begin{array}{l}{ }_{[(\mathrm{H} 2 \mathrm{O})} \mathrm{O} 6-\mathrm{H} 61 \cdots \mathrm{O} 10_{(\mathrm{C}=\mathrm{O})] 2} \&(\mathrm{NH}) \mathrm{N} 4-\mathrm{H} 33^{\cdots} \mathrm{O} 12_{(\mathrm{C}=\mathrm{O})} \\
*\left[(\mathrm{OH}) \mathrm{O} 9-\mathrm{H} 64 \cdots \mathrm{O} 1_{(\mathrm{C}=\mathrm{O})}\right]_{2} \&(\mathrm{NH}) \mathrm{N} 1-\mathrm{H} 2 \cdots \mathrm{O} 3_{(\mathrm{C}=\mathrm{O})} \\
{ }_{(\mathrm{OH})} \mathrm{O} 9-\mathrm{H} 64 \cdots \mathrm{O} 2_{(\mathrm{OH})} \&(\mathrm{NH}) \\
\mathrm{N} 1-\mathrm{H} 2 \cdots \mathrm{O} 3_{(\mathrm{C}=\mathrm{O})}\end{array}$ \\
\hline
\end{tabular}




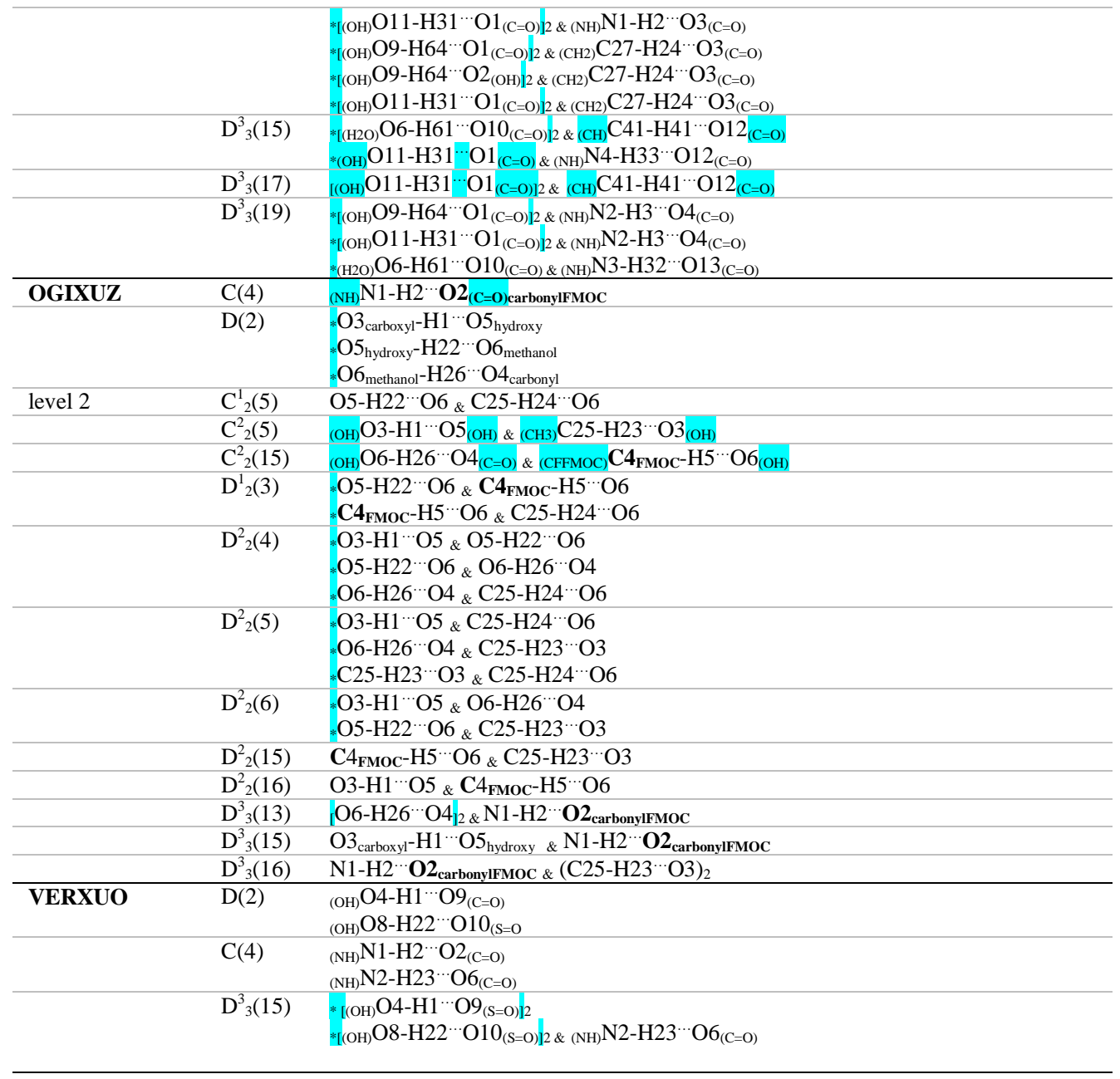

\begin{tabular}{|c|c|c|}
\hline \multirow[t]{5}{*}{ DULLAZ } & $S(6)$ & ${ }_{(\mathrm{CH} 2)} \mathrm{C} 4-\mathrm{H} 3 \cdots \mathrm{O} 4_{(\mathrm{C}=\mathrm{O})}$ \\
\hline & $S(7)$ & $\begin{array}{l}{ }^{(\mathrm{CH} 3)} \mathrm{C} 13-\mathrm{H} 13 \cdots \mathrm{O} 1_{(\mathrm{C}=\mathrm{O})} \\
{ }_{(\mathrm{CH} 3)} \mathrm{C} 41-\mathrm{H} 44 \cdots 5_{(\mathrm{C}=\mathrm{O})}\end{array}$ \\
\hline & $\mathrm{C}(5)$ & 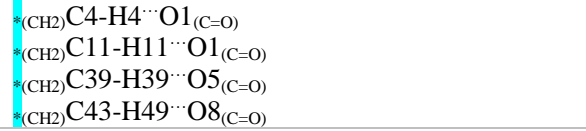 \\
\hline & $\mathrm{C}(6)$ & ${ }_{(\mathrm{CH})} \mathrm{C} 11-\mathrm{H} 10 \cdots 3_{(\mathrm{C}-\mathrm{O}-\mathrm{C})}$ \\
\hline & $\mathrm{D}(2)$ & 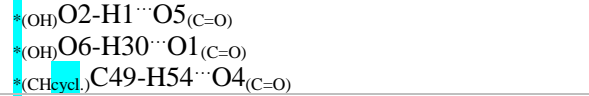 \\
\hline \multirow[t]{18}{*}{ Level 2} & $\mathrm{C}_{2}^{2}(10)$ & $\begin{array}{l}{ }_{(\mathrm{CH} 2)} \mathrm{C} 4-\mathrm{H} 4 \cdots \mathrm{O} 1_{(\mathrm{C}=\mathrm{O})} \&(\mathrm{CH} 2) \\
{ }_{(\mathrm{CH} 2)} \mathrm{C} 11-\mathrm{H} 11-\mathrm{H} 10 \cdots \mathrm{O} 3_{(\mathrm{C}-\mathrm{O}-\mathrm{C})} \\
{ }_{(\mathrm{C}-\mathrm{O}-\mathrm{C})} \&(\mathrm{CH} 2) \\
\mathrm{C} 11-\mathrm{H} 11^{\cdots} \mathrm{O} 1_{(\mathrm{C}=\mathrm{O})}\end{array}$ \\
\hline & $\mathrm{C}_{2}^{2}(11)$ & ${ }_{(\mathrm{CH} 2)} \mathrm{C} 11-\mathrm{H} 10 \cdots \mathrm{O} 3_{(\mathrm{C}-\mathrm{O}-\mathrm{C})} \&(\mathrm{CH} 2) \mathrm{C} 4-\mathrm{H} 4 \cdots \mathrm{O} 1_{(\mathrm{C}=\mathrm{O})}$ \\
\hline & $\mathrm{C}_{2}^{2}(14)$ & ${ }_{(\mathrm{CH} 2)} \mathrm{C} 39-\mathrm{H} 39 \cdots \mathrm{O}_{(\mathrm{C}=\mathrm{O})} \&\left(\mathrm{CH}_{2}\right) \mathrm{C} 43-\mathrm{H} 49 \cdots \mathrm{O} 8_{(\mathrm{C}=\mathrm{O})}$ \\
\hline & $\mathrm{C}_{2}^{2}(19)$ & 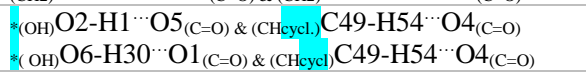 \\
\hline & $\mathrm{R}_{2}^{1}(6)$ & ${ }_{(\mathrm{CH} 2)} \mathrm{C} 4-\mathrm{H} 4 \cdots \mathrm{O} 1_{(\mathrm{C}=\mathrm{O})} \&\left(\mathrm{CH}_{2}\right) \mathrm{C} 11-\mathrm{H} 10 \cdots \mathrm{O} 3_{(\mathrm{C}-\mathrm{O}-\mathrm{C})}$ \\
\hline & $\mathrm{R}_{2}^{2}(8)$ & $(\mathrm{OH}) \mathrm{O} 2-\mathrm{H} 1 \cdots \mathrm{O} 5_{(\mathrm{C}=\mathrm{O})} \&(\mathrm{OH}) \mathrm{C} 6-\mathrm{H} 30 \cdots \mathrm{O} 1_{(\mathrm{C}=\mathrm{O})}$ \\
\hline & $\mathrm{R}_{2}^{2}(9)$ & ${ }_{(\mathrm{CH} 2)} \mathrm{C} 11-\mathrm{H} 11 \cdots \mathrm{O} 1_{(\mathrm{C}=\mathrm{O})} \&(\mathrm{CH} 2) \mathrm{C} 11-\mathrm{H} 10 \cdots \mathrm{O} 3_{(\mathrm{C}=\mathrm{O})}$ \\
\hline & $\mathrm{R}_{2}^{2}(11)$ & ${ }_{(\mathrm{CH} 2)} \mathrm{C} 4-\mathrm{H} 4 \cdots \mathrm{O} 1_{(\mathrm{C}=\mathrm{O})} \&(\mathrm{CH} 2)$ \\
\hline & $\mathrm{R}_{2}^{2}(14)$ & ${ }_{(\mathrm{CH} 2)} \mathrm{C} 39-\mathrm{H} 39 \cdots \mathrm{O} 5_{(\mathrm{C}=\mathrm{O})} \&\left(\mathrm{CH}_{2}\right) \mathrm{C} 43-\mathrm{H} 49 \cdots \mathrm{O} 8_{(\mathrm{C}=\mathrm{O})}$ \\
\hline & $\mathrm{D}_{3}^{2}(8)$ & $*(\mathrm{OH}) \mathrm{O} 6-\mathrm{H} 30 \cdots \mathrm{O} 1_{(\mathrm{C}=\mathrm{O})} \mid 2 \&{ }_{(\mathrm{CH} 2)} \mathrm{C} 11-\mathrm{H} 11 \cdots \mathrm{O} 1_{(\mathrm{C}=\mathrm{O})}$ \\
\hline & $\mathrm{D}_{3}^{3}(8)$ & $\begin{array}{l}*(\mathrm{OH}) \mathrm{O} 2-\mathrm{H} 1 \cdots \mathrm{O} 5_{(\mathrm{C}=\mathrm{O})} 2 \&(\mathrm{CH} 2) \mathrm{C} 39-\mathrm{H} 39 \cdots \mathrm{O} 5_{(\mathrm{C}=\mathrm{O})} \\
{ }_{(\mathrm{OH})} \mathrm{O} 6-\mathrm{H} 30 \cdots \mathrm{O} 1_{(\mathrm{C}=\mathrm{O})} \&(\mathrm{CH} 2) \\
\mathrm{C} 4-\mathrm{H} 4 \cdots \mathrm{O} 1_{(\mathrm{C}=\mathrm{O})}\end{array}$ \\
\hline & $\mathrm{D}_{3}^{3}(11)$ & ${ }_{(\mathrm{CH} 2)} \mathrm{C} 11-\mathrm{H} 10 \cdots \mathrm{O} 3_{(\mathrm{C}-\mathrm{O}-\mathrm{C})} \&\left(\mathrm{CH}\right.$ cycl) $\mathrm{C} 49-\mathrm{H} 54 \cdots \mathrm{O} 4_{(\mathrm{C}=\mathrm{O})}$ \\
\hline & $\mathrm{D}_{3}^{3}(12)$ & 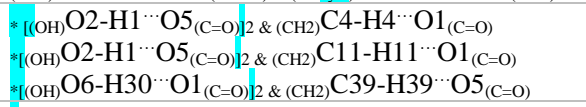 \\
\hline & $\mathrm{D}_{3}^{3}(13)$ & ${ }_{(\mathrm{OH})} \mathrm{O} 6-\mathrm{H} 30 \cdots 1_{(\mathrm{C}=\mathrm{O})} \&(\mathrm{CH} 2) \mathrm{C} 11-\mathrm{H} 10 \cdots 3_{(\mathrm{C}-\mathrm{O}-\mathrm{C})}$ \\
\hline & $\mathrm{D}_{3}^{3}(14)$ & 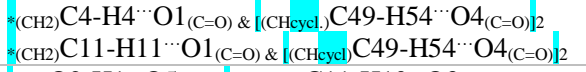 \\
\hline & $\mathrm{D}_{3}^{3}(15)$ & $(\mathrm{OH}) \mathrm{O} 2-\mathrm{H} 1 \cdots \mathrm{O} 5_{(\mathrm{C}=\mathrm{O})} \mid 2 \&(\mathrm{CH} 2) \mathrm{C} 11-\mathrm{H} 10 \cdots \mathrm{O} 3_{(\mathrm{C}-\mathrm{O}-\mathrm{C})}$ \\
\hline & $\mathrm{D}_{3}^{3}(16)$ & $\left({ }_{(\mathrm{OH})} \mathrm{O} 2-\mathrm{H} 1 \cdots 5_{(\mathrm{C}=\mathrm{O}) \mid 2} \&(\mathrm{CH} 2) \mathrm{C} 43-\mathrm{H} 49 \cdots \mathrm{O} 8_{(\mathrm{C}=\mathrm{O})}\right.$ \\
\hline & $\mathrm{D}_{3}^{3}(18)$ & 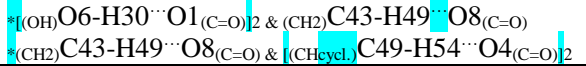 \\
\hline \multirow[t]{2}{*}{ MOXSUP } & $\mathrm{S}(6)$ & $\begin{array}{l}{ }_{(\mathrm{CH})} \mathrm{C} 18-\mathrm{H} 14 \cdots \mathrm{O} 2_{(\mathrm{C}=\mathrm{O})} \\
{ }_{(\mathrm{CH} 2)} \mathrm{C} 25-\mathrm{H} 22 \cdots \mathrm{O} 1_{(\mathrm{C}-\mathrm{O}-\mathrm{C})}\end{array}$ \\
\hline & $\mathrm{C}(5)$ & ${ }_{(\mathrm{CH} 2)} \mathrm{C} 2-\mathrm{H} 3 \cdots \mathrm{O} 2_{(\mathrm{C}=\mathrm{O})}$ \\
\hline
\end{tabular}




\begin{tabular}{|c|c|c|}
\hline & & $*_{(\mathrm{CH} 2)} \mathrm{C} 25-\mathrm{H} 21 \cdots \mathrm{O} 4_{(\mathrm{C}=0)}$ \\
\hline & $\mathrm{C}(6)$ & ${ }_{(\mathrm{CH} 2)} \mathrm{C} 25-\mathrm{H} 22 \cdots \mathrm{O} 1_{(\mathrm{C}-\mathrm{O}-\mathrm{C})}$ \\
\hline & $\mathrm{R}_{2}^{2}(8)$ & {$\left[(\mathrm{OH}) \mathrm{O} 3-\mathrm{H} 1 \cdots \mathrm{O} 4_{(\mathrm{C}=\mathrm{O})}\right]_{2}$} \\
\hline \multirow[t]{8}{*}{ Level 2} & $\mathrm{C}_{2}^{2}(11)$ & $\begin{array}{l}{ }_{(\mathrm{CH} 2)} \mathrm{C} 2-\mathrm{H} 3 \cdots \mathrm{O} 2_{(\mathrm{C}=\mathrm{O})} \&(\mathrm{CH} 2) \mathrm{C} 25-\mathrm{H} 22 \cdots \mathrm{O} 1_{(\mathrm{C}-\mathrm{O}-\mathrm{C})} \\
{ }_{(\mathrm{CH} 2)} \mathrm{C} 25-\mathrm{H} 21 \cdots \mathrm{O} 4_{(\mathrm{C}=\mathrm{O})} \&(\mathrm{CH} 2) \mathrm{C} 25-\mathrm{H} 22 \cdots \mathrm{O} 1_{(\mathrm{C}-\mathrm{O}-\mathrm{C})}\end{array}$ \\
\hline & $\mathrm{C}_{2}^{2}(14)$ & ${ }_{(\mathrm{CH} 2)} \mathrm{C} 2-\mathrm{H} 3 \cdots \mathrm{O} 2_{(\mathrm{C}=\mathrm{O})} \&(\mathrm{CH} 2) \mathrm{C} 25-\mathrm{H} 21 \cdots \mathrm{O} 4_{(\mathrm{C}=\mathrm{O})}$ \\
\hline & $\mathrm{R}_{2}^{2}(9)$ & $\begin{array}{l}{ }_{(\mathrm{CH} 2)} \mathrm{C} 2-\mathrm{H} 3 \cdots \mathrm{O} 2_{(\mathrm{C}=\mathrm{O})} \&(\mathrm{CH} 2) \mathrm{C} 25-\mathrm{H} 22 \cdots \mathrm{O} 1_{(\mathrm{C}-\mathrm{O}-\mathrm{C})} \\
{ }_{(\mathrm{CH} 2)} \mathrm{C} 25-\mathrm{H} 21 \cdots \mathrm{O} 4_{(\mathrm{C}=\mathrm{O})} \&(\mathrm{CH} 2) \mathrm{C} 25-\mathrm{H} 22 \cdots \mathrm{O} 1_{(\mathrm{C}-\mathrm{O}-\mathrm{C})}\end{array}$ \\
\hline & $\mathrm{R}_{2}^{2}(14)$ & ${ }_{(\mathrm{CH} 2)} \mathrm{C} 2-\mathrm{H} 3 \cdots \mathrm{O} 2_{(\mathrm{C}=\mathrm{O})} \&\left[(\mathrm{CH} 2) \mathrm{C} 25-\mathrm{H} 21 \cdots \mathrm{O} 4_{(\mathrm{C}=\mathrm{O})]_{2}}\right.$ \\
\hline & $\mathrm{R}_{4}^{2}(14)$ & {$\left[(\mathrm{OH}) \mathrm{O} 3-\mathrm{H} 1 \cdots \mathrm{O} 4_{(\mathrm{C}=\mathrm{O})}\right] 2 \&\left[(\mathrm{CH} 2) \mathrm{C} 25-\mathrm{H} 21 \cdots \mathrm{O} 4_{(\mathrm{C}=\mathrm{O})]_{2}}\right.$} \\
\hline & $\mathrm{C}_{4}^{3}(16)$ & {$\left[(\mathrm{OH}) \mathrm{O} 3-\mathrm{H} 1 \cdots \mathrm{O} 4_{(\mathrm{C}=\mathrm{O})}\right] 2 \&\left[(\mathrm{CH} 2) \mathrm{C} 25-\mathrm{H} 21^{\cdots} \mathrm{O} 4_{(\mathrm{C}=\mathrm{O})}\right]_{2}$} \\
\hline & $\mathrm{R}_{4}^{3}(16)$ & {$\left[(\mathrm{OH}) \mathrm{O} 3-\mathrm{H} 1 \cdots \mathrm{O} 4_{(\mathrm{C}=\mathrm{O})}\right] 2 \&\left[(\mathrm{CH} 2) \mathrm{C} 25-\mathrm{H} 21^{\cdots} \mathrm{O} 4_{(\mathrm{C}=\mathrm{O})}\right]_{2}$} \\
\hline & $\mathrm{R}_{4}^{4}(18)$ & {$\left[(\mathrm{OH}) \mathrm{O} 3-\mathrm{H} 1 \cdots \mathrm{O} 4_{(\mathrm{C}=\mathrm{O})}\right] 2 \&\left[(\mathrm{CH} 2) \mathrm{C} 25-\mathrm{H} 21 \cdots \mathrm{O} 4_{(\mathrm{C}=\mathrm{O})}\right] 2$} \\
\hline \multicolumn{3}{|r|}{ group $\boldsymbol{D}$} \\
\hline \multirow[t]{2}{*}{ UQIYUQ } & $\mathrm{C}(4)$ & ${ }_{(\mathrm{OH})} \mathrm{O} 4-\mathrm{H} 1^{\cdots} \mathrm{O} 3_{(\mathrm{C}=\mathrm{O})}$ \\
\hline & $C(5)$ & $\begin{array}{l}{ }_{(\mathrm{NH})} \mathrm{N} 1-\mathrm{H} 1 \cdots \mathrm{O} 4_{(\mathrm{OH})} \\
*_{(\mathrm{CH} 2)} \mathrm{C} 14-\mathrm{H} 13 \cdots \mathrm{O} 2_{(\mathrm{C}=\mathrm{O})} \\
{ }_{(\mathrm{CH})} \mathrm{C} 16-\mathrm{H} 14 \cdots \mathrm{O} 1_{(\mathrm{C}-\mathrm{O}-\mathrm{C})}\end{array}$ \\
\hline \multirow[t]{12}{*}{ Level 2} & $\mathrm{C}_{2}^{2}(7)$ & ${ }_{(\mathrm{OH})} \mathrm{O} 4-\mathrm{H} 1 \cdots \mathrm{O} 3_{(\mathrm{C}=\mathrm{O})} \&(\mathrm{NH}) \mathrm{N} 1-\mathrm{H} 2 \cdots \mathrm{O} 4_{(\mathrm{OH})}$ \\
\hline & $\mathrm{C}_{2}^{2}(9)$ & ${ }_{(\mathrm{OH})} \mathrm{O} 4-\mathrm{H} 1 \cdots \mathrm{O} 3_{(\mathrm{C}=\mathrm{O})} \&(\mathrm{NH}) \mathrm{N} 1-\mathrm{H} 2 \cdots \mathrm{O} 4_{(\mathrm{OH})}$ \\
\hline & $C_{2}^{2}(10)$ & $\begin{array}{l}*_{(\mathrm{NH})} \mathrm{N} 1-\mathrm{H} 2 \cdots \mathrm{O} 4_{(\mathrm{OH})} \&(\mathrm{CH}) \mathrm{C} 16-\mathrm{H} 14 \cdots \mathrm{O} 1_{(\mathrm{C}-\mathrm{O}-\mathrm{C})} \\
*_{(\mathrm{CH} 2)} \mathrm{C} 14-\mathrm{H} 13 \cdots \mathrm{O} 2_{(\mathrm{C}=\mathrm{O})} \&(\mathrm{CH}) \mathrm{C} 16-\mathrm{H} 14 \cdots \mathrm{O} 1_{(\mathrm{C}-\mathrm{O}-\mathrm{C})}\end{array}$ \\
\hline & $\mathrm{C}_{2}^{2}(11)$ & 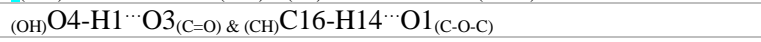 \\
\hline & $\mathrm{C}_{2}^{2}(12)$ & ${ }_{(\mathrm{NH})} \mathrm{N} 1-\mathrm{H} 2 \cdots \mathrm{O} 4_{(\mathrm{OH})} \&(\mathrm{CH} 2) \mathrm{C} 14-\mathrm{H} 13 \cdots \mathrm{O} 2_{(\mathrm{C}=\mathrm{O})}$ \\
\hline & $\mathrm{C}_{2}^{2}(15)$ & ${ }_{(\mathrm{OH})} \mathrm{O} 4-\mathrm{H} 1 \cdots \mathrm{O} 3_{(\mathrm{C}=\mathrm{O})} \&(\mathrm{CH} 2) \mathrm{C} 14-\mathrm{H} 13 \cdots \mathrm{O} 2_{(\mathrm{C}=\mathrm{O})}$ \\
\hline & $\mathrm{R}_{2}^{2}(8)$ & $\begin{array}{l}{ }_{(\mathrm{NH})} \mathrm{N} 1-\mathrm{H} 2 \cdots \mathrm{O} 4_{(\mathrm{OH})} \&(\mathrm{CH}) \mathrm{C} 16-\mathrm{H} 14 \cdots \mathrm{O} 1_{(\mathrm{C}-\mathrm{O}-\mathrm{C})} \\
{ }_{(\mathrm{CH} 2)} \mathrm{C} 14-\mathrm{H} 13 \cdots \mathrm{O} 2_{(\mathrm{C}=\mathrm{O})} \&(\mathrm{CH}) \\
\mathrm{C} 16-\mathrm{H} 14 \cdots \mathrm{O} 1_{(\mathrm{C}-\mathrm{O}-\mathrm{C})}\end{array}$ \\
\hline & $\mathrm{R}_{2}^{2}(11)$ & ${ }_{(\mathrm{NH})} \mathrm{N} 1-\mathrm{H} 2 \cdots \mathrm{O} 4_{(\mathrm{OH})} \&{ }_{(\mathrm{CH} 2)} \mathrm{C} 14-\mathrm{H} 13 \cdots \mathrm{O} 2_{(\mathrm{C}=\mathrm{O})}$ \\
\hline & $\mathrm{R}_{3}^{3}(11)$ & 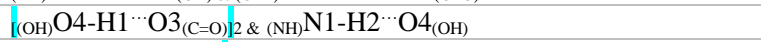 \\
\hline & $\mathrm{R}_{3}^{3}(15)$ & ${ }_{(\mathrm{OH})} \mathrm{O} 4-\mathrm{H} 1 \cdots \mathrm{O} 3_{(\mathrm{C}=\mathrm{O})} \mid 2 \&(\mathrm{CH}) \mathrm{C} 16-\mathrm{H} 14 \cdots \mathrm{O} 1_{(\mathrm{C}-\mathrm{O}-\mathrm{C})}$ \\
\hline & $\mathrm{R}_{3}^{3}(19)$ & ${ }_{(\mathrm{OH})} \mathrm{O} 4-\mathrm{H} 1 \cdots \mathrm{O} 3_{(\mathrm{C}=\mathrm{O})} \&(\mathrm{CH} 2) \mathrm{C} 14-\mathrm{H} 13 \cdots \mathrm{O} 2_{(\mathrm{C}=\mathrm{O})}$ \\
\hline & $\mathrm{R}_{4}^{4}(16)$ & {$\left[(\mathrm{OH}) \mathrm{O} 4-\mathrm{H} 1 \cdots \mathrm{O} 3_{(\mathrm{C}=\mathrm{O})}\right] 2 \&\left[(\mathrm{NH}) \mathrm{N} 1-\mathrm{H} 2 \cdots \mathrm{O} 4_{(\mathrm{OH})] 2}\right.$} \\
\hline UQOGUE & $\mathrm{C}(4)$ & $(\mathrm{OH}) \mathrm{O} 4_{\text {carboxyl }}-\mathrm{H} 1^{\cdots} \mathrm{O} 3_{(\mathrm{C}=0) \text { carboxyl }}$ \\
\hline \multirow[t]{5}{*}{ level 1} & $\mathrm{C}(5)$ & 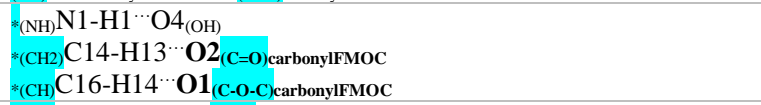 \\
\hline & $\mathrm{C}(6)$ & ${ }_{(\mathrm{CH} 2)} \mathrm{C} 24-\mathrm{H} 20 \cdots \mathrm{O6}_{(\mathrm{NO} 2)}$ \\
\hline & $\mathrm{C}(8)$ & ${ }_{(\mathrm{CH} 2)} \mathrm{C} 18-\mathrm{H} 16 \cdots 6_{(\mathrm{NO} 2)}$ \\
\hline & $\mathrm{C}(13)$ & $\mathrm{C} 14-\mathrm{H} 13 \cdots \mathrm{O} 5$ \\
\hline & $\mathrm{C}(18)$ & C10 $_{\text {FMOC }}-\mathrm{H} 9 \cdots \mathrm{O}$ \\
\hline \multirow[t]{22}{*}{ level 2} & $\mathrm{C}_{2}^{1}(14)$ & C10 $_{\text {FMOC }}-\mathrm{H} 9 \cdots \mathrm{O} 6$ \& $\mathrm{C} 18-\mathrm{H} 16 \cdots \mathrm{O} 6$ \\
\hline & $\mathrm{C}_{2}^{1}(16)$ & $\mathbf{C 1 0}_{\text {Fмос }}-\mathrm{H} 9 \cdots \mathrm{O} 6_{\&}$ C $24-\mathrm{H} 20 \cdots \mathrm{O} 6$ \\
\hline & $\mathrm{C}_{1}^{2}(11)$ & $\mathrm{C} 14-\mathrm{H} 13 \cdots \mathrm{O} 5$ \& $\mathrm{C} 14-\mathrm{H} 13 \cdots \mathbf{O} 2_{\text {carbonylFMoc }}$ \\
\hline & $\mathrm{C}_{2}^{2}(7)$ & ${ }_{(\mathrm{OH})} \mathrm{O} 4_{\text {carboxyl }}-\mathrm{H} 1 \cdots \mathrm{O} 3_{(\mathrm{C}=\mathrm{O}) \text { carboxyl } \&(\mathrm{NH})} \mathrm{N} 1-\mathrm{H} 1 \cdots{ }^{\cdots} 4_{(\mathrm{OH}) \text { carboxyl }}$ \\
\hline & $\mathrm{C}_{2}^{2}(9)$ & $\mathrm{O} 4_{\text {carboxyl }}-\mathrm{H} 1 \cdots \mathrm{O} 3_{\text {carboxyl \& }} \mathrm{N} 1-\mathrm{H} 1 \cdots \mathrm{O} 4_{\text {carboxyl }}$ \\
\hline & $\mathrm{C}_{2}^{2}(10)$ & $\begin{array}{l}{ }_{(\mathrm{NH})} \mathrm{N} 1-\mathrm{H} 2 \cdots \mathrm{O} 4_{(\mathrm{OH}) \text { carboxyl \& }(\mathrm{CH})} \mathrm{C} 16-\mathrm{H} 14 \cdots \mathbf{O 1}_{(\mathrm{C}-\mathrm{O}-\mathrm{C}) \text { carbonylFMOC }} \\
{ }_{*(\mathrm{CH} 2)} \mathrm{C} 14-\mathrm{H} 13 \cdots \mathbf{O}_{(\mathrm{C}=\mathrm{O}) \text { carbonylFMOC \& }} \mathrm{C} 16-\mathrm{H} 14 \cdots \mathbf{O 1}_{\text {carbonylFMOC }}\end{array}$ \\
\hline & $\mathrm{C}_{2}^{2}(11)$ & $\begin{array}{l}\mathrm{O} 4_{\text {carboxyl }}-\mathrm{H} 1 \cdots \mathrm{O} 3_{\text {carboxyl } \&} \mathrm{C} 16-\mathrm{H} 14 \cdots \mathbf{O 1}_{\text {carbonylFMOC }} \\
\text { C10 } 10_{\text {FMOC }}-\mathrm{H} 9 \cdots \mathrm{O} 6_{\&} \mathrm{C} 14-\mathrm{H} 13^{\cdots} \mathrm{O} 5 \\
\mathrm{C} 14-\mathrm{H} 13^{\cdots} \mathrm{O} 5_{\&} \mathrm{C} 18-\mathrm{H} 16^{\cdots} \mathrm{O} 6\end{array}$ \\
\hline & $\mathrm{C}_{2}^{2}(12)$ & $\begin{array}{l}\text { N1-H2 } \cdots \text { O } 4_{\text {carboxyl \& }} \mathrm{C} 14-\mathrm{H} 13 \cdots \mathbf{O}_{\text {carbonylFMoc }} \\
\mathrm{C} 14-\mathrm{H} 13 \cdots \mathrm{O} 5_{\&} \mathrm{C} 16-\mathrm{H} 14 \cdots \mathbf{O 1}_{\text {carbonylFMoc }}\end{array}$ \\
\hline & $\mathrm{C}_{2}^{2}(13)$ & ${ }_{(\mathrm{CH} 2)} \mathrm{C} 14-\mathrm{H} 13 \cdots \mathrm{O} 5_{(\mathrm{NO} 2)} \&(\mathrm{CHcycl}) \mathrm{C} 24-\mathrm{H} 20 \cdots \mathrm{O} 6_{(\mathrm{NO} 2)}$ \\
\hline & $\mathrm{C}_{2}^{2}(14)$ & $\mathrm{C} 18-\mathrm{H} 16 \cdots \mathrm{O} 6_{\&} \mathrm{C} 24-\mathrm{H} 20 \cdots \mathrm{O} 6$ \\
\hline & $\mathrm{C}_{2}^{2}(15)$ & $\begin{array}{l}\mathrm{O} 44_{\text {carboxyl }}-\mathrm{H} 1 \cdots \mathrm{O} 3_{\text {carboxyl \& }} \mathrm{C} 14-\mathrm{H} 13 \cdots \mathrm{O} 2_{\text {carbonylFMOc }} \\
\mathrm{N} 1-\mathrm{H} 2 \cdots \mathrm{O} 4_{\text {carboxyl \& }} \mathrm{C} 18-\mathrm{H} 16 \cdots \mathrm{O} 6 \\
\mathrm{C} 16-\mathrm{H} 14 \cdots \mathbf{O}_{\text {carbonylFMoc \& }} \mathrm{C} 18-\mathrm{H} 16 \cdots \mathrm{O} 6\end{array}$ \\
\hline & $\mathrm{C}_{2}^{2}(16)$ & $\begin{array}{l}{ }_{(\mathrm{OH})} \mathrm{O} 4_{\text {carboxyl }}-\mathrm{H} 1 \cdots \mathrm{O} 3_{(\mathrm{C}=\mathrm{O}) \text { carboxyl }} \&\left(\mathrm{CH}_{2}\right) \\
\left.{ }^{*} \mathrm{C} H\right) \\
\mathrm{N} 1-\mathrm{H} 2{ }^{\cdots} \mathrm{O} 4_{(\mathrm{OH})} \&{ }_{(\mathrm{CH} 2)} \mathrm{C} 14-\mathrm{H} 16 \cdots \mathrm{O}_{(\mathrm{NO} 2)}\end{array}$ \\
\hline & $\mathrm{C}_{2}^{2}(17)$ & $\begin{array}{l}{ }_{(\mathrm{NH})} \mathrm{N} 1-\mathrm{H} 2 \cdots \mathrm{O} 4{ }_{(\mathrm{OH})} \&(\mathrm{CHcycl}) \\
{ }_{(\mathrm{CHFMOC})} \mathrm{C10}_{\mathrm{FMOC}}-\mathrm{H} 9 \cdots \mathrm{O} 6_{(\mathrm{NO} 2)} \&{ }_{(\mathrm{CH})} \mathrm{C} 16-\mathrm{H} 14 \cdots \mathrm{O1}_{(\mathrm{C}-\mathrm{O}-\mathrm{C}) \text { carbonylFMOC }} \\
{ }_{(\mathrm{CH})} \mathrm{C} 16-\mathrm{H} 14 \cdots \mathbf{O 1}_{(\mathrm{C}-\mathrm{O}-\mathrm{C}) \text { carbonylFMOC } \&(\mathrm{CHcycl})} \mathrm{C} 24-\mathrm{H} 20 \cdots \mathrm{O} 6_{(\mathrm{NO} 2)}\end{array}$ \\
\hline & $\mathrm{C}_{2}^{2}(18)$ & $\begin{array}{l}\mathrm{O} 44_{\text {carboxyl }}-\mathrm{H} 1 \cdots \mathrm{O} 3_{\text {carboxyl \& }} \mathrm{C} 24-\mathrm{H} 20 \cdots \mathrm{O} 6 \\
\mathrm{C} 14-\mathrm{H} 13 \cdots \mathrm{O} 2_{\text {carbonylFMOc \& }} \mathrm{C} 14-\mathrm{H} 13 \cdots \mathrm{O} 5 \\
\mathrm{C} 14-\mathrm{H} 13 \cdots \mathbf{O} 2_{\text {carbonylFMOc \& }} \mathrm{C} 16-\mathrm{H} 14 \cdots \mathrm{O} 1_{\text {carbonylFMoc }}\end{array}$ \\
\hline & $\mathrm{C}_{2}^{2}(19)$ & 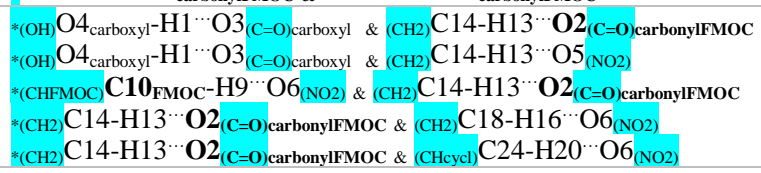 \\
\hline & $\mathrm{R}_{2}^{1}(6)$ & $\mathrm{C} 18-\mathrm{H} 16 \cdots \mathrm{O} 6$ \& $\mathrm{C} 24-\mathrm{H} 20 \cdots \mathrm{O} 6$ \\
\hline & $\mathrm{R}_{2}^{2}(8)$ & $\begin{array}{l}\text { N1-H2 } \cdots 4_{\text {carboxyl \& }} \mathrm{C} 16-\mathrm{H} 14 \cdots \mathbf{O 1}_{\text {carbonylFMoc }} \\
{ }^{\mathrm{C} 14-\mathrm{H} 13} \cdots \mathbf{O}_{\text {carbonylFMOC \& }} \mathrm{C} 16-\mathrm{H} 14 \cdots \mathbf{O 1}_{\text {carbonylFMoc }}\end{array}$ \\
\hline & $\mathrm{R}_{2}^{2}(12)$ & $\mathrm{N} 1-\mathrm{H} 2 \cdots \mathrm{O} 4_{\text {carboxyl \& }} \mathrm{C} 14-\mathrm{H} 13 \cdots \mathbf{O} 2_{\text {carbonylFMOC }}$ \\
\hline & $\mathrm{R}_{3}^{3}(11)$ & $*[\mathrm{O} 4-\mathrm{H} 1 \cdots \mathrm{O} 3]_{2} \& \mathrm{~N} 1-\mathrm{H} 2 \cdots \mathrm{O} 4$ \\
\hline & $\mathrm{R}_{3}^{3}(15)$ & {$[\mathrm{O} 4-\mathrm{H} 1 \cdots \mathrm{O} 3]_{2}$ \& $\mathrm{C} 16-\mathrm{H} 14 \cdots \mathbf{O 1}_{\text {carbonylFMoc }}$} \\
\hline & $\mathrm{R}_{3}^{3}(19)$ & {$[\mathrm{O} 4-\mathrm{H} 1 \cdots \mathrm{O} 3]_{2}$ \& $\mathrm{C} 14-\mathrm{H} 13 \cdots \mathbf{O 2}_{\text {carbonylFMOC }}$} \\
\hline & $\mathrm{R}_{4}^{4}(16)$ & {$[\mathrm{O} 4-\mathrm{H} 1 \cdots \mathrm{O} 3]_{2} \&[\mathrm{~N} 1-\mathrm{H} 2 \cdots \mathrm{O} 4]_{2}$} \\
\hline
\end{tabular}




\begin{tabular}{|c|c|c|}
\hline \multicolumn{3}{|r|}{ group $E$} \\
\hline XATJAG & $\mathrm{C}(4)$ & $\begin{array}{l}\mathrm{N} 1-\mathrm{H} 13 \cdots \mathbf{O}_{\text {carbonylFMOC }} \\
{ }_{(\mathrm{C}=\mathrm{O})} \mathrm{O} 3-\mathrm{H} 20 \cdots \mathrm{O} 4 \\
(\mathrm{C}=\mathrm{O})\end{array}$ \\
\hline & $\mathrm{C}(5)$ & ${ }_{(\mathrm{CH} 2)} \mathrm{C} 21-\mathrm{H} 16 \cdots \mathrm{O} 4_{(\mathrm{C}=\mathrm{O})}$ \\
\hline \multirow[t]{4}{*}{ level 2} & $\mathrm{C}_{2}^{2}(9)$ & $(\mathrm{C}=0) \mathrm{O} 3-\mathrm{H} 20 \cdots \mathrm{O} 4_{(\mathrm{C}=0) 1 \&} \mathrm{C} 21-\mathrm{H} 16 \cdots \mathrm{O} 4_{(\mathrm{C}=0)}$ \\
\hline & $\mathrm{C}_{2}^{2}(11)$ & $\mathrm{N} 1-\mathrm{H} 13 \cdots \mathbf{O}_{\text {carbonylFMOc \& }} \mathrm{C} 21-\mathrm{H} 16 \cdots \mathrm{O} 4_{(\mathrm{C}=\mathrm{O})}$ \\
\hline & $\mathrm{C}_{2}^{2}(12)$ & $\mathrm{N} 1-\mathrm{H} 13 \cdots \mathrm{O} 2_{\text {carbonylFMOC \& }} \mathrm{O} 3_{\text {carboxyl}}-\mathrm{H} 20 \cdots \mathrm{O} 4_{(\mathrm{C}=0)}$ \\
\hline & $\mathrm{R}_{2}^{1}(7)$ & $(\mathrm{C}=0) \mathrm{O} 3-\mathrm{H} 20 \cdots \mathrm{O} 4_{(\mathrm{C}=0) \&} \mathrm{C} 21-\mathrm{H} 16 \cdots \mathrm{O} 4_{(\mathrm{C}=\mathrm{O})}$ \\
\hline \multirow[t]{2}{*}{ XАТJEK } & $\mathrm{C}(4)$ & $\begin{array}{l}{ }^{*}(\mathrm{OH}) \mathrm{O} 2-\mathrm{H} 1_{\text {carboxyl }} \cdots{ }^{\prime \prime} 4_{(\mathrm{C}=\mathrm{O})} \\
{ }_{(\mathrm{NH})} \mathrm{N} 1-\mathrm{H} 2 \cdots \cdots 3_{(\mathrm{C}=\mathrm{O}) \text { carbonylFMOC }}\end{array}$ \\
\hline & $\mathrm{C}(13)$ & 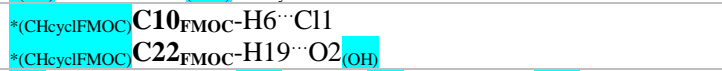 \\
\hline \multirow[t]{5}{*}{ level 2} & $\mathrm{C}_{2}^{2}(12)$ & (OH) $\mathrm{O} 2-\mathrm{H} 1_{\text {carboxyl }} \cdots 4_{(\mathrm{C}=\mathrm{O}) \text { carboxyl \& (NH) }} \mathrm{N} 1-\mathrm{H} 2 \cdots 3_{(\mathrm{C}=\mathrm{O}) \text { carbonylFMOC }}$ \\
\hline & $\mathrm{C}^{2}{ }_{2}(15)$ & $\begin{array}{l}\text { "(OH) } \mathrm{O} 2-\mathrm{H} 1_{\text {carboxyl }} \cdots \mathrm{O}_{\text {carboxyl \& }} \mathbf{C 2 2}_{\mathrm{FMOC}}-\mathrm{H} 19 \cdots \mathrm{O} 2_{(\mathrm{OH})} \\
{ }^{(\mathrm{NHH})} \mathrm{N} 1-\mathrm{H} 2 \cdots 3_{\text {carbonylFOC \& }} \mathbf{C 1 0}_{\mathrm{FMOC}}-\mathrm{H} 6 \cdots \mathrm{Cl} 1 \\
{ }^{(\mathrm{NH})} \mathrm{N} 1-\mathrm{H} 2 \cdots 3_{\text {carbonylFMOC \& }} \mathbf{C 2 2}_{\mathrm{FMOC}}-\mathrm{H} 19 \cdots \mathrm{O} 2_{(\mathrm{OH})}\end{array}$ \\
\hline & $\mathrm{C}_{2}^{2}(16)$ & (CH) $\mathrm{C} 10-\mathrm{H} 6 \cdots \mathrm{Cl} 1_{(\mathrm{CHFMOC})} \mathbf{C 2 2}_{\mathrm{FMOC}}-\mathrm{H} 19 \cdots \mathrm{O} 2_{(\mathrm{OH})}$ \\
\hline & $\mathrm{C}_{2}^{2}(17)$ & 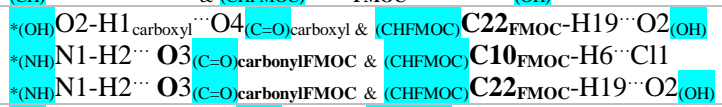 \\
\hline & $\mathrm{C}_{2}^{2}(19)$ & $(\mathrm{OH}) \mathrm{O} 2-\mathrm{H} 1_{\text {carboxyl }}{ }^{\circ} 4_{(\mathrm{C}=\mathrm{O}) \text { carboxyl \& (CHFMOC) }} \mathbf{C 1 0}_{\mathrm{FMOC}}-\mathrm{H} 6 \cdots \mathrm{Cl}$ \\
\hline \multirow[t]{3}{*}{ XATKEL } & $\mathrm{C}(4)$ & $\begin{array}{l}{ }^{(\mathrm{NH})} \mathrm{N} 1-\mathrm{H} 13 \cdots 2_{(\mathrm{C}=\mathrm{O}) \text { carbonylFMOC }} \\
\mathrm{O} 3_{(\mathrm{OH}) \text { carboxyl }}-\mathrm{H} 20 \cdots 4_{(\mathrm{C}=\mathrm{O}) \text { carboxyl }}\end{array}$ \\
\hline & $\mathrm{C}(5)$ & ${ }_{(\mathrm{CH} 2)} \mathrm{C} 21-\mathrm{H} 16 \cdots \mathrm{O} 4_{(\mathrm{C}=\mathrm{O})}$ \\
\hline & $\mathrm{C}(16)$ & С9 9MOC-H6”I1 \\
\hline \multirow[t]{7}{*}{ level 2} & $\mathrm{C}_{2}^{2}(9)$ & $\mathrm{O} 3-\mathrm{H} 20 \cdots \mathrm{O} 4 \& \mathrm{C} 21-\mathrm{H} 16 \cdots \mathrm{O} 4$ \\
\hline & $\mathrm{C}_{2}^{2}(11)$ & 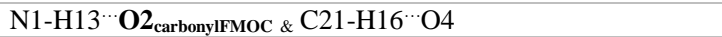 \\
\hline & $\mathrm{C}_{2}^{2}(12)$ & $(\mathrm{OH}) \mathrm{O} 3-\mathrm{H} 20 \cdots \mathrm{O} 4_{(\mathrm{C}=\mathrm{O})} \&(\mathrm{NH}) \mathrm{N} 1-\mathrm{H} 13 \cdots \mathbf{O}_{(\mathrm{C}=\mathrm{O}) \text { carbonylFMOC }}$ \\
\hline & $\mathrm{C}_{2}^{2}(18)$ & N1-H13 $\cdots \mathbf{O 2}_{\text {carbonylFMOC \& }} \mathbf{C 9}_{\text {FMOC }}-\mathrm{H} 6 \cdots$ I1 \\
\hline & $\mathrm{C}_{2}^{2}(19)$ & ${ }_{(\mathrm{CH} 2)} \mathrm{C} 21-\mathrm{H} 16 \cdots \mathrm{O} 4_{(\mathrm{C}=\mathrm{O})} \&(\mathrm{CHFMOC}) \mathbf{C} 9_{\mathrm{FMOC}}-\mathrm{H} 6 \cdots \mathrm{I} 1$ \\
\hline & $\mathrm{C}_{2}^{2}(20)$ & (NH) $\mathrm{N} 1-\mathrm{H} 13 \cdots \mathbf{O 2}_{(\mathrm{C}=\mathrm{O}) \text { carbonylFMOC \& (CHFMOC) }} \mathbf{C 9}_{\mathrm{FMOC}}-\mathrm{H} 6 \cdots \mathrm{I} 1$ \\
\hline & $\mathrm{R}_{2}^{1}(7)$ & $\mathrm{O} 3-\mathrm{H} 20 \cdots \mathrm{O} 4$ \& $\mathrm{C} 21-\mathrm{H} 16 \cdots \mathrm{O} 4$ \\
\hline \multirow[t]{2}{*}{ XАTKIP } & $\mathrm{C}(4)$ & N1-H12 $\cdots \mathbf{O 2}_{\text {carbonylFMOC }}$ \\
\hline & $\mathrm{D}(2)$ & $\mathrm{O}_{\text {carboxyl- }} \mathrm{H} 14 \cdots \mathrm{O} 5$ \\
\hline \multirow[t]{4}{*}{ level 2} & $\mathrm{C}_{2}^{2}(8)$ & ${ }_{(\mathrm{OH})} \mathrm{O} 3-\mathrm{H} 14 \cdots \mathrm{O} 5_{(\mathrm{S}=\mathrm{O})} \&(\mathrm{CH} 3) \mathrm{C} 25-\mathrm{H} 30 \cdots \mathrm{O} 4_{(\mathrm{C}=\mathrm{O})}$ \\
\hline & $\mathrm{D}_{3}^{3}(7)$ & $\mathrm{O} 3-\mathrm{H} 14 \cdots \mathrm{O} 5$ \& $\mathrm{C} 26-\mathrm{H} 24 \cdots \mathrm{O} 5$ \\
\hline & $\mathrm{D}^{3}{ }_{3}(11)$ & $(\mathrm{C} 25-\mathrm{H} 30 \cdots \mathrm{O} 4)_{2} \& \mathrm{C} 26-\mathrm{H} 24 \cdots \mathrm{O} 5$ \\
\hline & $\mathrm{D}_{3}^{3}(15)$ & 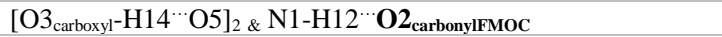 \\
\hline
\end{tabular}

\section{Fmoc-ALANINE derivatives}

\begin{tabular}{|c|c|c|}
\hline \multirow{4}{*}{ ADAGUK } & $\mathrm{C}(7)$ & ${ }_{(\mathrm{OH})} \mathrm{O} 4-\mathrm{H} 22 \cdots \mathrm{O} 2_{(\mathrm{C}=\mathrm{O})}$ \\
\hline & $\mathrm{C}(8)$ & $\left(\mathrm{CH}\right.$ cycl) $\mathrm{C} 22-\mathrm{H} 15 \cdots 3_{(\mathrm{C}=\mathrm{O})}$ \\
\hline & $\mathrm{C}(12)$ & $(\mathrm{CH}$ cycl $) \mathrm{C} 5-\mathrm{H} 4 \cdots 3_{(\mathrm{C}=\mathrm{O})}$ \\
\hline & $\mathrm{C}(13)$ & ${ }_{(\mathrm{CHcycl})} \mathrm{C} 9-\mathrm{H} 6 \cdots \mathrm{O} 4_{(\mathrm{OH})}$ \\
\hline \multirow[t]{7}{*}{ Level 2} & $\mathrm{C}_{2}{ }_{2}(16)$ & $(\mathrm{CHcycl}) \mathrm{C} 5-\mathrm{H} 4 \cdots \mathrm{O} 3_{(\mathrm{C}=\mathrm{O})} \&(\mathrm{CHcycl}) \mathrm{C} 22-\mathrm{H} 15 \cdots \mathrm{O} 3_{(\mathrm{C}=\mathrm{O})}$ \\
\hline & $\mathrm{C}_{2}^{2}(10)$ & ${ }_{(\mathrm{CHcyc})} \mathrm{C} 5-\mathrm{H} 4 \cdots 3_{(\mathrm{C}=\mathrm{O})} \&(\mathrm{CHcycl}) \mathrm{C} 9-\mathrm{H} 6 \cdots 4_{(\mathrm{OH})}$ \\
\hline & $\mathrm{C}_{2}^{2}(12)$ & $(\mathrm{OH}) \mathrm{O} 4-\mathrm{H} 22 \cdots \mathrm{O} 2_{(\mathrm{C}=\mathrm{O})} \&(\mathrm{Ccycl}) \mathrm{C} 9-\mathrm{H} 6 \cdots \mathrm{O} 4_{(\mathrm{OH})}$ \\
\hline & $\mathrm{C}_{2}^{2}(13)$ & $\begin{array}{l}{ }^{*(\mathrm{OH})} \mathrm{O} 4-\mathrm{H} 22 \cdots \mathrm{O} 2_{(\mathrm{C}=\mathrm{O})} \&(\mathrm{Ccycl}) \mathrm{C} 5-\mathrm{H} 4 \cdots \mathrm{O} 3_{(\mathrm{C}=\mathrm{O})} \\
{ }^{(\mathrm{OH})} \mathrm{O} 4-\mathrm{H} 22 \cdots \mathrm{O} 2_{(\mathrm{C}=\mathrm{O})} \&(\mathrm{CH} \mathrm{Cycl}) \\
\mathrm{C} 22-\mathrm{H} 15 \cdots \mathrm{O} 3_{(\mathrm{C}=\mathrm{O})}\end{array}$ \\
\hline & $\mathrm{C}_{2}^{2}(15)$ & ${ }_{(\mathrm{OH})} \mathrm{O} 4-\mathrm{H} 22 \cdots \mathrm{O} 2_{(\mathrm{C}=\mathrm{O})} \&(\mathrm{CHcycl}) \mathrm{C} 22-\mathrm{H} 15 \cdots \mathrm{O} 3_{(\mathrm{C}=\mathrm{O})}$ \\
\hline & $\mathrm{C}_{2}^{2}(19)$ & $\begin{array}{l}{ }_{(\mathrm{CH} \text { (Cycl) }} \mathrm{C} 5-\mathrm{H} 4 \cdots \mathrm{O} 3_{(\mathrm{C}=\mathrm{O})} \&(\mathrm{OH}) \mathrm{O} 4-\mathrm{H} 22 \cdots \mathrm{O} 2_{(\mathrm{C}=\mathrm{O})} \\
{ }_{(\mathrm{CHcycl})} \mathrm{C} 9-\mathrm{H} 6 \cdots \mathrm{O} 4_{(\mathrm{OH})} \&(\mathrm{CHcycl}) \mathrm{C} 22-\mathrm{H} 15 \cdots \mathrm{O} 3_{(\mathrm{C}=\mathrm{O})}\end{array}$ \\
\hline & $\mathrm{C}_{2}^{2}(20)$ & $\begin{array}{l}{ }^{(\mathrm{CH} C \mathrm{cycl})} \mathrm{C} 5-\mathrm{H} 4 \cdots \mathrm{O} 3_{(\mathrm{C}=\mathrm{O})} \&(\mathrm{CHcycl}) \\
{ }_{(\mathrm{OH})} \mathrm{O} 4-\mathrm{H} 22 \cdots \mathrm{O} 22-\mathrm{H} 15 \cdots \mathrm{O} 3_{(\mathrm{C}=\mathrm{O})} \&(\mathrm{CHcycl}) \\
\mathrm{C} 9-\mathrm{H} 6 \cdots\end{array}$ \\
\hline \multirow[t]{4}{*}{ CUWKOU01 } & $\mathrm{S}(6)$ & $(\mathrm{CH}) \mathrm{C} 19-\mathrm{H} 21 \cdots \mathrm{O} 2_{(\mathrm{C}=\mathrm{O})}$ \\
\hline & $\mathrm{C}(4)$ & $(\mathrm{NH}) \mathrm{N} 1-\mathrm{H} 2 \cdots \mathrm{O} 3_{(\mathrm{C}=\mathrm{O})}$ \\
\hline & $C(7)$ & $\left.{ }_{(\mathrm{CHcycl})}\right) \mathrm{C} 3-\mathrm{H} 4 \cdots \mathrm{O}_{(\mathrm{C}-\mathrm{O}-\mathrm{C})}$ \\
\hline & $\mathrm{C}(9)$ & ${ }_{(\mathrm{CH} \text { cycl. })} \mathrm{C} 3-\mathrm{H} 4 \cdots \mathrm{O} 2_{(\mathrm{C}=\mathrm{O})}$ \\
\hline \multirow[t]{8}{*}{ Level 2} & $\mathrm{R}_{2}^{2}(8)$ & $(\mathrm{OH}) \mathrm{O} 3-\mathrm{H} 1 \cdots \mathrm{O} 3_{(\mathrm{OH})} \&(\mathrm{OH}) \mathrm{O} 4-\mathrm{H} 20 \cdots \mathrm{O} 4_{(\mathrm{OH})}$ \\
\hline & $\mathrm{R}_{3}^{4}(20)$ & $\mid(\mathrm{CH}$ cycl. $) \mathrm{C} 3-\mathrm{H} 4 \cdots \mathrm{O} 2_{(\mathrm{C}=\mathrm{O}) \mid 2} \& \mid\left(\mathrm{CH}\right.$ cycl. $\mathrm{C} 3-\left.\mathrm{H} 4 \cdots \mathrm{O} 1_{(\mathrm{C}-\mathrm{O}-\mathrm{C})}\right|_{2}$ \\
\hline & $\mathrm{C}_{2}^{1}(11)$ & $(\mathrm{CHcycl}.) \mathrm{C} 3-\mathrm{H} 4 \cdots \mathrm{O} 2_{(\mathrm{C}=\mathrm{O})} \&(\mathrm{OH}) \mathrm{O} 4-\mathrm{H} 2 \cdots \mathrm{O} 4_{(\mathrm{OH})}$ \\
\hline & $\mathrm{C}_{1}^{2}(4)$ & ${ }_{(\mathrm{CHcycl})} \mathrm{C} 3-\mathrm{H} 4 \cdots \mathrm{O} 1_{(\mathrm{C}-\mathrm{O}-\mathrm{C})} \&(\mathrm{CHcycl}) \mathrm{C} 3-\mathrm{H} 4 \cdots \mathrm{O} 2_{(\mathrm{C}=\mathrm{O})}$ \\
\hline & $\mathrm{C}_{2}^{2}(13)$ & $\begin{array}{l}{ }_{(\mathrm{NH})} \mathrm{N} 1-\mathrm{H} 2 \cdots \mathrm{O} 2_{(\mathrm{C}=\mathrm{O})} \&(\mathrm{CHcycl.}) \\
{ }_{(\mathrm{CHcycl})} \mathrm{C} 3-\mathrm{C} 3-\mathrm{H} 4 \cdots \cdots \mathrm{O} 2_{(\mathrm{C}=\mathrm{O})} 2_{(\mathrm{C}=\mathrm{O})} \&(\mathrm{NH}) \\
\mathrm{N} 1-\mathrm{H} 2 \cdots \mathrm{O} 2_{(\mathrm{C}=\mathrm{O})}\end{array}$ \\
\hline & $\mathrm{C}_{2}^{2}(16)$ & $\left({ }_{(\mathrm{CHcycl} .)} \mathrm{C} 3-\mathrm{H} 4 \cdots \mathrm{O} 2_{(\mathrm{C}=\mathrm{O})} \&(\mathrm{CHcycl}) \mathrm{C} 3-\mathrm{H} 4 \cdots \mathrm{O} 1_{(\mathrm{C}-\mathrm{O}-\mathrm{C})}\right.$ \\
\hline & $\mathrm{C}^{2}{ }_{2}(19)$ & 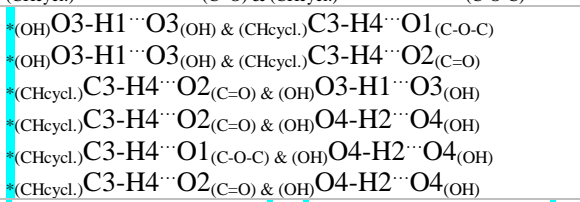 \\
\hline & $\mathrm{C}_{3}^{4}(20)$ & {$\left[(\mathrm{CHcycl}) \mathrm{C} 3-\mathrm{H} 4 \cdots \mathrm{O} 1_{(\mathrm{C}-\mathrm{O}-\mathrm{C})}\right]_{2} \&\left[(\mathrm{CHcycl}) \mathrm{C} 3-\mathrm{H} 4 \cdots \mathrm{O} 2_{(\mathrm{C}=\mathrm{O})]_{2}}\right.$} \\
\hline \multicolumn{3}{|l|}{ CUWKIO01 } \\
\hline & $\mathrm{D}(2)$ & $\begin{array}{l}{ }_{(\mathrm{OH})} \mathrm{O} 4-\mathrm{H} 1 \cdots \mathrm{O} 5_{(\mathrm{H} 2 \mathrm{O})} \\
{ }_{(\mathrm{H} 2 \mathrm{O})} \mathrm{O} 5-\mathrm{H} 18 \cdots \mathrm{O} 2_{(\mathrm{C}=\mathrm{O})} \\
{ }_{(\mathrm{H} 2 \mathrm{O})} \mathrm{O} 5-\mathrm{H} 19 \cdots 3_{(\mathrm{C}=\mathrm{O})}\end{array}$ \\
\hline & $\mathrm{C}(5)$ & ${ }_{(\mathrm{NH})} \mathrm{N} 1-\mathrm{H} 2 \cdots \mathrm{O} 3_{(\mathrm{C}=\mathrm{O})}$ \\
\hline & $\mathrm{C}(9)$ & ${ }_{(\mathrm{CHcycl})} \mathrm{C} 3-\mathrm{H} 4 \cdots \mathrm{O} 2_{(\mathrm{C}=\mathrm{O})}$ \\
\hline
\end{tabular}




\begin{tabular}{|c|c|c|}
\hline & $\mathrm{D}_{3}^{2}(8)$ & {$\left[(\mathrm{H} 20) \mathrm{O} 5-\mathrm{H} 19 \cdots \mathrm{O} 3_{(\mathrm{C}=\mathrm{O})] 2} \&(\mathrm{NH}) \mathrm{N} 1-\mathrm{H} 2 \cdots \mathrm{O} 3_{(\mathrm{C}=\mathrm{O})}\right.$} \\
\hline & $\mathrm{C}_{2}^{2}(6)$ & {$\left[(\mathrm{OH}) \mathrm{O} 5-\mathrm{H} 19 \cdots \mathrm{O} 3_{(\mathrm{C}=\mathrm{O})}\right]_{2}$} \\
\hline & $\mathrm{C}_{2}^{2}(9)$ & $\begin{array}{l}{ }_{(\mathrm{OH} 2)} \mathrm{O} 5-\mathrm{H} 18^{\cdots} \mathrm{O} 2_{(\mathrm{C}=\mathrm{O})} \&(\mathrm{OH}) \mathrm{O} 4-\mathrm{H} 1 \cdots \mathrm{O}_{5(\mathrm{H} 2 \mathrm{O})} \\
{ }^{*(\mathrm{H} 2 \mathrm{O})} \mathrm{O} 5-\mathrm{H} 18^{\cdots} \mathrm{O} 2_{\&[(\mathrm{H} 2 \mathrm{O})} \mathrm{O} 5-\mathrm{H} 19 \cdots \mathrm{O} 3_{(\mathrm{C}=\mathrm{O})] 2}\end{array}$ \\
\hline & $\mathrm{C}_{2}^{2}(16)$ & ${ }_{(\mathrm{NH})} \mathrm{N} 1-\mathrm{H} 2 \cdots \mathrm{O} 3_{(\mathrm{C}=\mathrm{O})} \&(\mathrm{CHcycl}) \mathrm{C} 3-\mathrm{H} 4 \cdots \mathrm{O} 2_{(\mathrm{C}=\mathrm{O})}$ \\
\hline \multirow[t]{2}{*}{ Level 2} & $\mathrm{D}_{3}^{3}(12)$ & 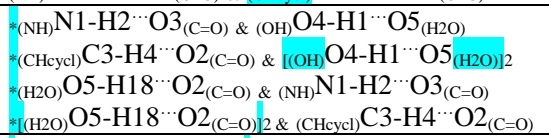 \\
\hline & $\mathrm{D}_{3}^{3}(20)$ & {$\left[(\mathrm{H} 20) \mathrm{O} 5-\mathrm{H} 19 \cdots \mathrm{O} 3_{(\mathrm{C}=\mathrm{O})}\right] 2 \&(\mathrm{CHcycl}) \mathrm{C} 3-\mathrm{H} 4 \cdots \mathrm{O} 2_{(\mathrm{C}=\mathrm{O})}$} \\
\hline \multicolumn{3}{|r|}{ Fmoc GLYCINE derivatives } \\
\hline \multirow[t]{4}{*}{ NOVTOJ } & $\mathrm{C}(4)$ & $(\mathrm{NH}) \mathrm{N} 1-\mathrm{H} 2 \cdots \mathrm{O} 5_{(\mathrm{C}=\mathrm{O})}$ \\
\hline & $\mathrm{C}_{2}^{2}(6)$ & ${ }_{(\mathrm{OH})} \mathrm{O} 3-\mathrm{H} 1 \cdots \mathrm{O} 7_{(\mathrm{C}=\mathrm{O})} \&(\mathrm{OH}) \mathrm{O} 7-\mathrm{H} 36 \cdots 6_{(\mathrm{C}=\mathrm{O})}$ \\
\hline & $\mathrm{D}(2)$ & $\begin{array}{l}*_{(\mathrm{OH})} \mathrm{O} 3-\mathrm{H} 1 \cdots \mathrm{O} 7_{(\mathrm{OH})} \\
*_{(\mathrm{OH})} \mathrm{O} 7-\mathrm{H} 36 \cdots 6_{(\mathrm{C}=\mathrm{O})}\end{array}$ \\
\hline & $\mathrm{D}_{3}^{3}(19)$ & ${ }_{(\mathrm{NH})} \mathrm{N} 1-\mathrm{H} 2 \cdots \mathrm{O} 5_{(\mathrm{C}=\mathrm{O})} \&\left[(\mathrm{OH}) \mathrm{O} 7-\mathrm{H} 36 \cdots 6_{(\mathrm{C}=\mathrm{O})]_{2}}\right.$ \\
\hline \multirow[t]{4}{*}{ VERQER } & $\mathrm{S}(7)$ & $(\mathrm{CH} 2) \mathrm{C} 16-\mathrm{H} 14 \cdots \mathrm{F} 5$ \\
\hline & $\mathrm{C}(8)$ & $\left(\mathrm{CH}_{c y c l}\right) \mathrm{C} 3-\mathrm{H} 3 \cdots \mathrm{O} 2_{(\mathrm{C}=\mathrm{O})}$ \\
\hline & $\mathrm{C}(9)$ & $\begin{array}{l}{ }_{(\mathrm{CHcycl})} \mathrm{C} 1-\mathrm{H} 2 \cdots \mathrm{O} 44_{(\mathrm{OH})} \\
{ }_{(\mathrm{CH} 2)} \mathrm{C} 14-\mathrm{H} 12 \cdots \mathrm{F} 5\end{array}$ \\
\hline & $\mathrm{C}(12)$ & $(\mathrm{CHcycl}) \mathrm{C} 4-\mathrm{H} 4 \cdots \mathrm{O} 4_{(\mathrm{OH})}$ \\
\hline \multirow[t]{12}{*}{ Level 2} & $\mathrm{C}_{2}^{1}(7)$ & 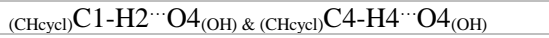 \\
\hline & $\mathrm{C}_{2}^{2}(10)$ & ${ }_{(\mathrm{CHcycl})} \mathrm{C} 3-\mathrm{H} 3 \cdots \mathrm{O} 2_{(\mathrm{C}=\mathrm{O})} \&(\mathrm{CHcycl}) \mathrm{C} 4-\mathrm{H} 4 \cdots \mathrm{O} 4_{(\mathrm{OH})}$ \\
\hline & $\mathrm{C}_{2}^{2}(12)$ & 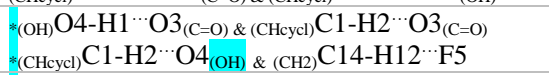 \\
\hline & $\mathrm{C}_{2}^{2}(13)$ & $\begin{array}{l}{ }_{(\mathrm{OH})} \mathrm{O} 4-\mathrm{H} 1 \cdots \mathrm{O} 3_{(\mathrm{C}=\mathrm{O})} \&(\mathrm{CHcycl}) \\
{ }_{(\mathrm{CHcycl})} \mathrm{C} 3-\mathrm{H} 3{ }^{\cdots} \mathrm{O} 22_{(\mathrm{C}=\mathrm{O})} \&(\mathrm{CH} 2) \\
\mathrm{C} 14-\mathrm{H} 12 \cdots \mathrm{F} 5\end{array}$ \\
\hline & $\mathrm{C}_{2}^{2}(14)$ & 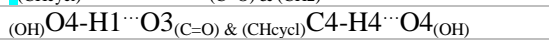 \\
\hline & $\mathrm{C}_{2}^{2}(15)$ & ${ }_{(\mathrm{CHcycl})} \mathrm{C} 4-\mathrm{H} 4 \cdots \mathrm{O} 4_{(\mathrm{OH})} \&(\mathrm{CH} 2)$ \\
\hline & $\mathrm{C}_{2}^{2}(16)$ & 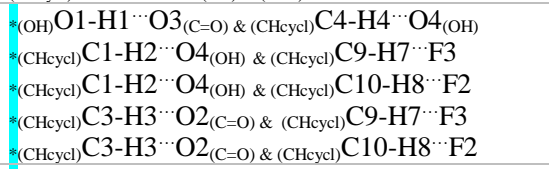 \\
\hline & $\mathrm{C}_{2}^{2}(17)$ & $\begin{array}{l}{ }_{(\mathrm{CHcycl})} \mathrm{C} 1-\mathrm{H} 2 \cdots \mathrm{O} 4_{(\mathrm{OH})} \&(\mathrm{CHcycl}) \\
{ }_{(\mathrm{CH} y \mathrm{Cl} l)} \mathrm{C} 3-\mathrm{H} 3{ }^{\cdots} \mathrm{O} 2^{\cdots}{ }_{(\mathrm{C}=\mathrm{O})} \&{ }_{(\mathrm{CH} 2)} \mathrm{C} 14-\mathrm{H} 12 \cdots \mathrm{F} 5\end{array}$ \\
\hline & $\mathrm{C}_{2}^{2}(18)$ & 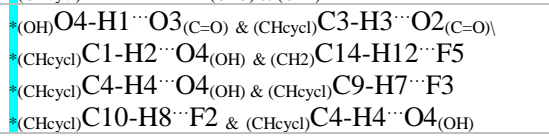 \\
\hline & $\mathrm{C}_{2}^{2}(20)$ & ${ }_{(\mathrm{CHcycl})} \mathrm{C} 3-\mathrm{H} 3 \cdots \mathrm{O} 2_{(\mathrm{C}=\mathrm{O})} \&(\mathrm{CHcycl}) \mathrm{C} 4-\mathrm{H} 4 \cdots \mathrm{O} 4_{(\mathrm{OH})}$ \\
\hline & $\mathrm{R}_{2}^{2}(8)$ & $\begin{array}{l}{ }^{*(\mathrm{OH})} \mathrm{O} 4-\mathrm{H} 1 \cdots \mathrm{O} 3_{(\mathrm{C}=\mathrm{O}) 12} \\
{ }_{(\mathrm{CHcycl})} \mathrm{C} 9-\mathrm{H} 7 \cdots \mathrm{F} 3_{\&(\mathrm{CHcycl})} \mathrm{C} 10-\mathrm{H} 8 \cdots \mathrm{F} 2\end{array}$ \\
\hline & $\mathrm{R}_{2}^{2}(11)$ & 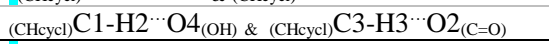 \\
\hline \multirow[t]{3}{*}{ VERQIW } & $\mathrm{D}(2)$ & 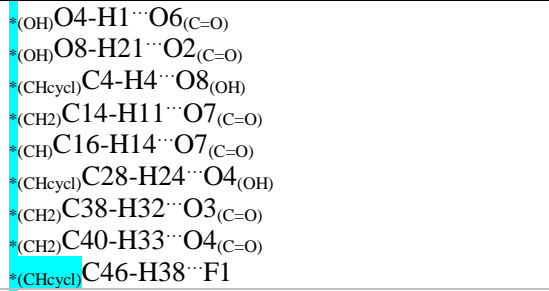 \\
\hline & $\mathrm{C}(8)$ & $\begin{array}{l}*(\mathrm{CHcycl}) \mathrm{C} 3-\mathrm{H} 3 \cdots \mathrm{O} 2_{(\mathrm{C}=\mathrm{O})} \\
*_{(\mathrm{CHcycl})} \mathrm{C} 27-\mathrm{H} 23 \cdots \mathrm{O} 6_{(\mathrm{C}=\mathrm{O})}\end{array}$ \\
\hline & $\mathrm{C}(14)$ & $\begin{array}{l}*(\mathrm{CHcycl}) \mathrm{C} 6-\mathrm{H} 6{ }^{\cdots} \mathrm{F} 1 \\
{ }_{(\mathrm{CH} \text { cycl })} \mathrm{C} 30-\mathrm{H} 26 \cdots \mathrm{F} 2\end{array}$ \\
\hline \multirow[t]{9}{*}{ Level 2} & $\mathrm{C}_{2}^{1}(8)$ & $\begin{array}{l}{ }_{(\mathrm{CH} 2)} \mathrm{C} 14-\mathrm{H} 11 \cdots \mathrm{O} 7_{(\mathrm{C}=\mathrm{O})} \&(\mathrm{CH}) \mathrm{C} 16-\mathrm{H} 14 \cdots \mathrm{O} 7_{(\mathrm{C}=\mathrm{O})} \\
{ }_{(\mathrm{CH} 2)} \mathrm{C} 38-\mathrm{H} 32^{\cdots} \mathrm{O} 3_{(\mathrm{C}=\mathrm{O})} \&(\mathrm{CH} 2) \mathrm{C} 40-\mathrm{H} 33^{\cdots} 3_{(\mathrm{C}=\mathrm{O})}\end{array}$ \\
\hline & $\mathrm{C}_{2}^{2}(8)$ & ${ }_{(\mathrm{CH})} \mathrm{C} 16-\mathrm{H} 14 \cdots \mathrm{O} 7_{(\mathrm{C}=\mathrm{O})} \&(\mathrm{CH} 2) \mathrm{C} 40-\mathrm{H} 33 \cdots \mathrm{O} 3_{(\mathrm{C}=\mathrm{O})}$ \\
\hline & $\mathrm{C}_{2}^{2}(9)$ & $\begin{array}{l}*(\mathrm{OH}) \mathrm{O} 4-\mathrm{H} 1 \cdots 6_{(\mathrm{C}=\mathrm{O})} \&(\mathrm{CHcycl}) \\
{ }_{(\mathrm{OH})} \mathrm{O} 4-\mathrm{H} 1 \cdots 6_{(\mathrm{C}=\mathrm{O})} \&(\mathrm{CH} 2) \\
*\end{array}$ \\
\hline & $\mathrm{C}_{2}^{2}(10)$ & $\begin{array}{l}{ }_{(\mathrm{CH} y c l)} \mathrm{C} 4-\mathrm{H} 4 \cdots \mathrm{O} 8_{(\mathrm{OH})} \&(\mathrm{CH} 2) \mathrm{C} 14-\mathrm{H} 11 \cdots \mathrm{O} 7_{(\mathrm{C}=\mathrm{O})} \\
{ }_{(\mathrm{CH} \text { cycl })} \mathrm{C} 28-\mathrm{H} 24 \cdots \mathrm{O} 4_{(\mathrm{OH})} \&(\mathrm{CH} 2) \mathrm{C} 38-\mathrm{H} 32 \cdots \mathrm{O} 3_{(\mathrm{C}=\mathrm{O})}\end{array}$ \\
\hline & $\mathrm{C}_{2}^{2}(11)$ & $\begin{array}{l}{ }_{(\mathrm{OH})} \mathrm{O} 4-\mathrm{H} 1 \cdots \mathrm{O} 6_{(\mathrm{C}=\mathrm{O})} \&(\mathrm{CH}) \\
{ }_{(\mathrm{OH})} \mathrm{O} 16-\mathrm{H} 1 \cdots \mathrm{O} 14 \cdots \mathrm{O} 6_{(\mathrm{C}=\mathrm{O})} \& \&(\mathrm{CHcycl}) \mathrm{C} 28-\mathrm{H} 24 \cdots \mathrm{O} 4_{(\mathrm{OH})} \\
{ }_{(\mathrm{CH}) \mathrm{Cycl})} \mathrm{C} 4-\mathrm{H} 4 \cdots \mathrm{O} 8_{(\mathrm{OH})} \&(\mathrm{OH}) \mathrm{O} 8-\mathrm{H} 21 \cdots \mathrm{O} 2_{(\mathrm{C}=\mathrm{O})} \\
{ }_{(\mathrm{OH})} \mathrm{O} 8-\mathrm{H} 21 \cdots \mathrm{O} 2_{(\mathrm{C}=\mathrm{O})} \&(\mathrm{CH} 2) \mathrm{C} 40-\mathrm{H} 33 \cdots \mathrm{O} 3_{(\mathrm{C}=\mathrm{O})}\end{array}$ \\
\hline & $\mathrm{C}_{2}^{2}(12)$ & $\begin{array}{l}{ }_{(\mathrm{CH} 2)} \mathrm{C} 14-\mathrm{H} 11 \cdots \mathrm{O} 7_{(\mathrm{C}=\mathrm{O})} \&(\mathrm{CH} 2) \mathrm{C} 40-\mathrm{H} 33 \cdots \mathrm{O} 3_{(\mathrm{C}=\mathrm{O})} \\
{ }_{(\mathrm{CH})} \mathrm{C} 16-\mathrm{H} 14 \cdots \mathrm{O} 7_{(\mathrm{C}=\mathrm{O})} \&(\mathrm{CH} 2) \mathrm{C} 38-\mathrm{H} 32 \cdots \mathrm{O} 3_{(\mathrm{C}=\mathrm{O})}\end{array}$ \\
\hline & $\mathrm{C}_{2}^{2}(14)$ & 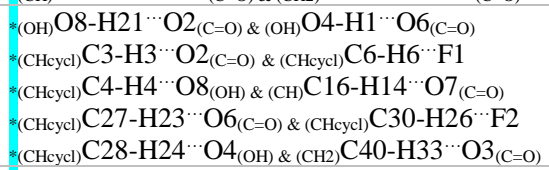 \\
\hline & $\mathrm{C}_{2}^{2}(15)$ & $\begin{array}{l}{ }_{(\mathrm{OH})}^{\mathrm{O}} 4-\mathrm{H} 1 \cdots \mathrm{O}_{(\mathrm{C}=\mathrm{O})} \&(\mathrm{CH} 2) \mathrm{C} 14-\mathrm{H} 11 \cdots \mathrm{O} 7_{(\mathrm{C}=\mathrm{O})} \\
{ }_{(\mathrm{OH})} \mathrm{O} 8-\mathrm{H} 21^{\cdots}{ }^{\circ} 2_{(\mathrm{C}=\mathrm{O})} \&(\mathrm{CH} 2) \\
\mathrm{C} 38-\mathrm{H} 32 \cdots \mathrm{O} 3_{(\mathrm{C}=\mathrm{O})}\end{array}$ \\
\hline & $C_{2}^{2}(16)$ & $\begin{array}{l}*_{(\mathrm{CH} y c l)} \mathrm{C} 4-\mathrm{H} 4 \cdots \mathrm{O} 8_{(\mathrm{OH})} \&(\mathrm{CH} 2) \mathrm{C} 40-\mathrm{H} 33 \cdots \mathrm{O} 3_{(\mathrm{C}=\mathrm{O})} \\
{ }_{(\mathrm{CH} 2)} \mathrm{C} 14-\mathrm{H} 11 \cdots \mathrm{O} 7_{(\mathrm{C}=\mathrm{O})} \&(\mathrm{CH} 2) \\
\mathrm{C} 38-\mathrm{H} 32 \cdots 3_{(\mathrm{C}=\mathrm{O})}\end{array}$ \\
\hline
\end{tabular}




\begin{tabular}{|c|c|c|}
\hline & & 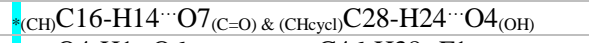 \\
\hline & $C_{2}^{2}(19)$ & 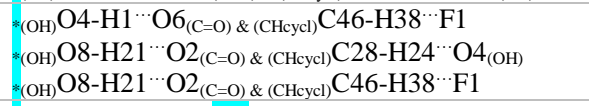 \\
\hline & $C_{2}^{2}(20)$ & $\begin{array}{l}{ }_{(\mathrm{CHcycl})} \mathrm{C} 4-\mathrm{H} 4 \cdots \mathrm{O} 8_{(\mathrm{OH})} \&(\mathrm{CH} 2) \mathrm{C} 38-\mathrm{H} 32 \cdots \mathrm{O} 3_{(\mathrm{C}=\mathrm{O})} \\
{ }_{(\mathrm{CH} 2)} \mathrm{C} 14-\mathrm{H} 11 \cdots \mathrm{O} 7_{(\mathrm{C}=\mathrm{O})} \&(\mathrm{CHcycl}) \mathrm{C} 28-\mathrm{H} 24 \cdots \mathrm{O} 4_{(\mathrm{OH})} \\
{ }_{(\mathrm{CH} 2)} \mathrm{C} 14-\mathrm{H} 11 \cdots \mathrm{O} 7_{(\mathrm{C}=\mathrm{O})} \&(\mathrm{CHcycl}) \\
\mathrm{C} 46-\mathrm{H} 38 \cdots \mathrm{F} 1 \\
{ }_{(\mathrm{CH} 2)} \mathrm{C} 38-\mathrm{H} 32 \cdots \mathrm{O} 3_{(\mathrm{C}=\mathrm{O})} \&(\mathrm{CHcycl}) \\
\mathrm{C} 46-\mathrm{H} 38 \cdots \mathrm{F} 1\end{array}$ \\
\hline & $\mathrm{R}_{2}^{2}(9)$ & $\begin{array}{l}{ }_{(\mathrm{OH})} \mathrm{O} 4-\mathrm{H} 1 \cdots \mathrm{O} 6_{(\mathrm{C}=\mathrm{O})} \&(\mathrm{CH} 2) \mathrm{C} 38-\mathrm{H} 32 \cdots \mathrm{O} 3_{(\mathrm{C}=\mathrm{O})} \\
{ }_{(\mathrm{OH})} \mathrm{O} 8-\mathrm{H} 21 \cdots \mathrm{O} 2_{(\mathrm{C}=\mathrm{O})} \&(\mathrm{CH} 2) \\
\mathrm{C} 14-\mathrm{H} 11 \cdots \mathrm{O} 7_{(\mathrm{C}=\mathrm{O})}\end{array}$ \\
\hline & $\mathrm{D}_{3}^{2}(11)$ & $\begin{array}{l}*\left[(\mathrm{OH}) \mathrm{O} 4-\mathrm{H} 1 \cdots \mathrm{O} 6_{(\mathrm{C}=\mathrm{O})} \&\right] 2 \&(\mathrm{CHcycl}) \\
\mathrm{C} 2\end{array}$ \\
\hline & $\mathrm{D}_{3}^{2}(19)$ & 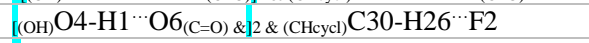 \\
\hline & $\mathrm{D}_{3}^{3}(13)$ & $\begin{array}{l}{ }_{(\mathrm{CHcycl})} \mathrm{C} 3-\mathrm{H} 3{ }^{\cdots \mathrm{O}} 2_{(\mathrm{C}=\mathrm{O})} \&(\mathrm{CH} 2) \mathrm{C} 14-\mathrm{H} 11 \cdots \mathrm{O} 7_{(\mathrm{C}=\mathrm{O})} \\
{ }_{(\mathrm{CHcycl})} \mathrm{C} 27-\mathrm{H} 23^{\cdots} \mathrm{O} 6_{(\mathrm{C}=\mathrm{O})} \&[(\mathrm{CH} 2) \\
\left.\mathrm{C} 38-\mathrm{H} 32^{\cdots} \mathrm{O} 3_{(\mathrm{C}=\mathrm{O})}\right]\end{array}$ \\
\hline & $\mathrm{D}_{3}^{3}(15)$ & $\begin{array}{l}{ }_{(\mathrm{CHcycl})} \mathrm{C} 3-\mathrm{H} 3 \cdots \mathrm{O} 2_{(\mathrm{C}=\mathrm{O})} \&[(\mathrm{CHcycl}) \\
\left.{ }_{(\mathrm{CH}} \mathrm{CH} 4-\mathrm{H} 4\right)\end{array}$ \\
\hline & $\mathrm{D}_{3}^{3}(17)$ & 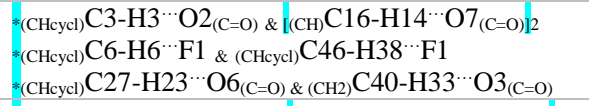 \\
\hline & $\mathrm{D}_{3}^{3}(19)$ & 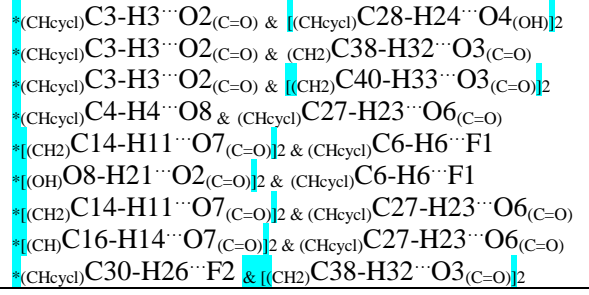 \\
\hline VERQOB & $\mathrm{R}_{2}^{1}(7)$ & ${ }_{(\mathrm{CH} 2)} \mathrm{C} 14-\mathrm{H} 11^{\cdots} \mathrm{F} 3$ \& (CHcycl) $\mathrm{C} 3-\mathrm{H} 3 \cdots \mathrm{F} 3$ \\
\hline & $\mathrm{R}_{2}^{2}(8)$ & {$\left[(\mathrm{OH}) \mathrm{O} 4-\mathrm{H} 1 \cdots \mathrm{O} 3_{(\mathrm{C}=\mathrm{O})}\right]_{2}$} \\
\hline & $\mathrm{R}_{2}^{2}(9)$ & ${ }_{(\mathrm{CH} 2)} \mathrm{C} 14-\mathrm{H} 12 \cdots \mathrm{O} 3_{(\mathrm{C}=\mathrm{O})} \&\left({ }_{(\mathrm{CH} 2)} \mathrm{C} 16-\mathrm{H} 13 \cdots \mathrm{O} 2_{(\mathrm{C}=\mathrm{O})}\right.$ \\
\hline & $\mathrm{R}_{2}^{2}(16)$ & {$[(\mathrm{CH} 2) \mathrm{C} 16-\mathrm{H} 14 \cdots \mathrm{F} 2]_{2}$} \\
\hline & $\mathrm{R}_{4}^{2}(20)$ & $(\mathrm{OH}) \mathrm{O} 4-\mathrm{H} 1 \cdots \mathrm{O} 3_{(\mathrm{C}=\mathrm{O})} \&(\mathrm{CH} 2) \mathrm{C} 14-\mathrm{H} 12 \cdots \mathrm{O} 3_{(\mathrm{C}=\mathrm{O})}$ \\
\hline & $\mathrm{R}_{2}^{3}(12)$ & $(\mathrm{H} 2 \mathrm{O}) \mathrm{O} 5-\mathrm{H} 18 \cdots \mathrm{F} 1$ \& $(\mathrm{CH} 2) \mathrm{C} 16-\mathrm{H} 14 \cdots \mathrm{F} 2$ \\
\hline & $\mathrm{R}_{4}^{4}(20)$ & {$\left[(\mathrm{CHcycl}) \mathrm{C} 3-\mathrm{H} 3{ }^{\cdots} \mathrm{F} 3\right]_{2 \&[(\mathrm{CHcycl})} \mathrm{C} 5-\mathrm{H} 5 \cdots \mathrm{F} 5_{\left.\right|_{2}}$} \\
\hline & $\mathrm{C}(5)$ & ${ }_{(\mathrm{CH} 2)} \mathrm{C} 16-\mathrm{H} 13 \cdots \mathrm{O} 2_{(\mathrm{C}=\mathrm{O})}$ \\
\hline & $\mathrm{C}(8)$ & ${ }_{(\mathrm{CH} 2)} \mathrm{C} 14-\mathrm{H} 12 \cdots \mathrm{O} 3_{(\mathrm{C}=\mathrm{O})}$ \\
\hline & $\mathrm{C}(11)$ & $(\mathrm{CH} 2) \mathrm{C} 14-\mathrm{H} 11 \cdots \mathrm{F} 3$ \\
\hline & $\mathrm{C}(14)$ & $\begin{array}{l}*(\mathrm{CHcycl}) \mathrm{C} 3-\mathrm{H} 3 \cdots \mathrm{F} 3 \\
{ }_{(\mathrm{CH} \text { cycl })} \mathrm{C} 5-\mathrm{H} 5 \cdots \mathrm{F} 5\end{array}$ \\
\hline & $C_{2}^{2}(11)$ & $\begin{array}{l}{ }_{(\mathrm{CH} 2)} \mathrm{C} 14-\mathrm{H} 11 \cdots \mathrm{F} 3{ }_{\&(\mathrm{CH} 2)} \mathrm{C} 16-\mathrm{H} 14-\mathrm{F} 2 \\
{ }_{(\mathrm{CH} 2)} \mathrm{C} 16-\mathrm{H} 14-\mathrm{F} 2{ }_{(\mathrm{CH} 2)} \mathrm{C} 16-\mathrm{H} 13 \cdots \mathrm{O} 2_{(\mathrm{C}=\mathrm{O})}\end{array}$ \\
\hline & $\mathrm{C}_{2}^{2}(13)$ & $\begin{array}{l}{ }_{(\mathrm{CH} 2)} \mathrm{C} 14-\mathrm{H} 12 \cdots \mathrm{O} 3_{(\mathrm{C}=\mathrm{O})} \&(\mathrm{CH} 2) \mathrm{C} 16-\mathrm{H} 13 \cdots \mathrm{O} 2_{(\mathrm{C}=\mathrm{O})} \\
{ }_{(\mathrm{CH} 2)} \mathrm{C} 16-\mathrm{H} 14-\mathrm{F} 2 \text { \& }{ }_{(\mathrm{CH} 2)} \mathrm{C} 16-\mathrm{H} 13 \cdots \mathrm{O} 2_{(\mathrm{C}=\mathrm{O})}\end{array}$ \\
\hline & $\mathrm{C}_{2}^{2}(14)$ & $\begin{array}{l}{ }_{(\mathrm{OH})} \mathrm{O} 4-\mathrm{H} 1 \cdots \mathrm{O} 3_{(\mathrm{C}=\mathrm{O})} \&(\mathrm{CH} 2) \mathrm{C} 16-\mathrm{H} 14-\mathrm{F} 2 \\
{ }_{(\mathrm{CHcycl})} \mathrm{C} 3-\mathrm{H} 3{ }^{\cdots} \mathrm{F} 3_{\&}{ }_{(\mathrm{CH} 2)} \mathrm{C} 16-\mathrm{H} 14-\mathrm{F} 2 \\
{ }_{(\mathrm{CH} 2)} \mathrm{C} 14-\mathrm{H} 12 \cdots \mathrm{O} 3_{(\mathrm{C}=\mathrm{O})} \&(\mathrm{CH} 2) \mathrm{C} 16-\mathrm{H} 14-\mathrm{F} 2\end{array}$ \\
\hline & $\mathrm{C}_{2}^{2}(16)$ & $\begin{array}{l}{ }_{(\mathrm{CH} 2)} \mathrm{C} 14-\mathrm{H} 12 \cdots \mathrm{O} 3_{(\mathrm{C}=\mathrm{O})} \&(\mathrm{CHcycl}) \mathrm{C} 5-\mathrm{H} 5 \cdots \mathrm{F} 5 \\
{ }_{(\mathrm{CH} 2)} \mathrm{C} 14-\mathrm{H} 11 \cdots \mathrm{F} 3_{\&(\mathrm{CH} 2)} \mathrm{C} 16-\mathrm{H} 13 \cdots \mathrm{O} 2_{(\mathrm{C}=\mathrm{O})} \\
{ }_{(\mathrm{CH} 2)} \mathrm{C} 14-\mathrm{H} 12 \cdots \mathrm{O} 3_{(\mathrm{C}=\mathrm{O})} \&(\mathrm{CH} 2) \mathrm{C} 16-\mathrm{H} 14-\mathrm{F} 2\end{array}$ \\
\hline & $\mathrm{C}_{2}^{2}(17)$ & $(\mathrm{CHcycl}) \mathrm{C} 5-\mathrm{H} 5 \cdots \mathrm{F} 5 \&(\mathrm{CH} 2) \mathrm{C} 16-\mathrm{H} 13 \cdots \mathrm{O} 2_{(\mathrm{C}=\mathrm{O})}$ \\
\hline & $\mathrm{C}_{2}^{2}(19)$ & 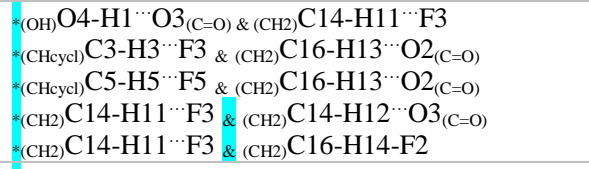 \\
\hline & $\mathrm{C}_{2}^{3}(15)$ & $\begin{array}{l}*(\mathrm{H} 2 \mathrm{O}) \mathrm{O} 5-\mathrm{H} 18 \cdots \mathrm{F} 1_{\&(\mathrm{CH} 2)} \mathrm{C} 14-\mathrm{H} 11 \cdots \mathrm{F} 3 \\
*\left[(\mathrm{H} 2 \mathrm{O}) \mathrm{O} 5-\mathrm{H} 18 \cdots \mathrm{F} 1_{\mid 2 \&(\mathrm{CH} 2)} \mathrm{C} 16-\mathrm{H} 13 \cdots \mathrm{O} 2\right.\end{array}$ \\
\hline & $\mathrm{C}_{2}^{3}(18)$ & $\begin{array}{l}*_{(\mathrm{H} 2 \mathrm{O})} \mathrm{O} 5-\mathrm{H} 18 \cdots \mathrm{F} 1_{22} \&(\mathrm{CHcycl}) \mathrm{C} 3-\mathrm{H} 3 \cdots \mathrm{F} 3 \\
{ }_{(\mathrm{CH} 2)} \mathrm{C} 14-\mathrm{H} 12 \cdots \mathrm{O} 3_{(\mathrm{C}=\mathrm{O})} \&[(\mathrm{H} 2 \mathrm{O}) \\
\mathrm{O} 5-\mathrm{H} 18^{\cdots}{ }^{\cdots} 1_{12}\end{array}$ \\
\hline & $\mathrm{D}(2)$ & $(\mathrm{H} 2 \mathrm{O}) \mathrm{O} 5-\mathrm{H} 18 \cdots \mathrm{F} 1$ \\
\hline & $\mathrm{D}^{2}{ }_{1}(3)$ & {$\left[(\mathrm{H} 2 \mathrm{O}) \mathrm{O} 5-\mathrm{H} 18^{\cdots} \mathrm{F} 1_{2}\right.$} \\
\hline VERXIC & $\mathrm{C}(4)$ & ${ }_{(\mathrm{CH} 2)} \mathrm{C} 16-\mathrm{H} 13 \cdots \mathrm{O} 3_{(\mathrm{C}=\mathrm{O})}$ \\
\hline & $\mathrm{C}(7)$ & ${ }_{(\mathrm{OH})} \mathrm{O} 4-\mathrm{H} 1 \cdots \mathrm{O} 2_{(\mathrm{C}=\mathrm{O})}$ \\
\hline & $\mathrm{C}(8)$ & $\begin{array}{l}{ }_{(\mathrm{CHcycl})} \mathrm{C} 3-\mathrm{H} 3 \cdots \mathrm{O} 2_{(\mathrm{C}=\mathrm{O})} \\
*_{(\mathrm{CH} 2)} \mathrm{C} 14-\mathrm{H} 12 \cdots \mathrm{O} 3_{(\mathrm{C}=\mathrm{O})}\end{array}$ \\
\hline & $\mathrm{C}(12)$ & ${ }_{(\mathrm{CHcycl})} \mathrm{C} 4-\mathrm{H} 4 \cdots \mathrm{O} 4_{(\mathrm{OH})}$ \\
\hline Level 2 & $\mathrm{C}_{2}^{1}(3)$ & $(\mathrm{OH}) \mathrm{O} 4-\mathrm{H} 1 \cdots \mathrm{O} 2_{(\mathrm{C}=\mathrm{O})} \&(\mathrm{CHcycl}) \mathrm{C} 3-\mathrm{H} 3 \cdots \mathrm{O} 2_{(\mathrm{C}=\mathrm{O})}$ \\
\hline & $\mathrm{C}_{2}^{1}(8)$ & ${ }_{(\mathrm{CH} 2)} \mathrm{C} 16-\mathrm{H} 13 \cdots \mathrm{O} 3_{(\mathrm{C}=\mathrm{O})} \&(\mathrm{CH} 2) \mathrm{C} 14-\mathrm{H} 12 \cdots \mathrm{O} 3_{(\mathrm{C}=\mathrm{O})}$ \\
\hline & $\mathrm{C}_{2}^{2}(9)$ & $(\mathrm{OH}) \mathrm{O} 4-\mathrm{H} 1 \cdots \mathrm{O} 2_{(\mathrm{C}=\mathrm{O})} \&(\mathrm{CH} 2) \mathrm{C} 16-\mathrm{H} 13 \cdots \mathrm{O} 3_{(\mathrm{C}=\mathrm{O})}$ \\
\hline & $\mathrm{C}_{2}^{2}(10)$ & $\begin{array}{l}{ }_{(\mathrm{CHcycl})} \mathrm{C} 3-\mathrm{H} 3 \cdots \mathrm{O} 2_{(\mathrm{C}=\mathrm{O})} \&(\mathrm{CHcycl}) \\
{ }_{(\mathrm{CH} y \mathrm{Cl})} \mathrm{C} 4-\mathrm{H} 4-\mathrm{H} 4 \cdots \mathrm{O} 4^{\cdots} 4_{(\mathrm{OH})} \&(\mathrm{CH} 2) \\
\mathrm{C} 14-\mathrm{H} 12 \cdots \mathrm{O} 3_{(\mathrm{C}=\mathrm{O})}\end{array}$ \\
\hline & $\mathrm{C}_{2}^{2}(11)$ & $\begin{array}{l}{ }_{(\mathrm{OH})} \mathrm{O} 4-\mathrm{H} 1 \cdots \mathrm{O} 2_{(\mathrm{C}=\mathrm{O})} \&(\mathrm{CHcycl}) \mathrm{C} 4-\mathrm{H} 4 \cdots \mathrm{O} 4_{(\mathrm{OH})} \\
{ }_{(\mathrm{OH})} \mathrm{O} 4-\mathrm{H} 1 \cdots \mathrm{O} 2_{(\mathrm{C}=\mathrm{O})} \&(\mathrm{CH} 2) \\
\mathrm{C} 16-\mathrm{H} 13 \cdots \mathrm{O} 3_{(\mathrm{C}=\mathrm{O})}\end{array}$ \\
\hline & $\mathrm{C}_{2}^{2}(12)$ & $\begin{array}{l}{ }_{(\mathrm{CHcycl})} \mathrm{C} 3-\mathrm{H} 3 \cdots \mathrm{O} 2_{(\mathrm{C}=\mathrm{O})} \&(\mathrm{CH} 2) \mathrm{C} 14-\mathrm{H} 12 \cdots \mathrm{O} 3_{(\mathrm{C}=\mathrm{O})} \\
{ }_{(\mathrm{CH} 2)} \mathrm{C} 14-\mathrm{H} 12 \cdots \mathrm{O} 3_{(\mathrm{C}=\mathrm{O})} \&(\mathrm{CH} 2) \mathrm{C} 16-\mathrm{H} 13 \cdots \mathrm{O} 3_{(\mathrm{C}=\mathrm{O})}\end{array}$ \\
\hline & $\mathrm{C}_{2}^{2}(14)$ & ${ }_{(\mathrm{CHcycl})} \mathrm{C} 4-\mathrm{H} 4 \cdots \mathrm{O} 4_{(\mathrm{OH})} \&(\mathrm{CH} 2)$ \\
\hline & $C_{2}^{2}(15)$ & $\begin{array}{l}{ }_{(\mathrm{OH})} \mathrm{O} 4-\mathrm{H} 1 \cdots \mathrm{O} 2_{(\mathrm{C}=\mathrm{O})} \&(\mathrm{CHcycl}) \\
\left.{ }^{*} \mathrm{CH}\right)\end{array}$ \\
\hline & $\mathrm{C}_{2}^{2}(16)$ & $*_{(\mathrm{CHcycl})} \mathrm{C} 3-\mathrm{H} 3 \cdots \mathrm{O} 2_{(\mathrm{C}=\mathrm{O})} \&(\mathrm{CH} 2) \mathrm{C} 14-\mathrm{H} 12 \cdots \mathrm{O} 3_{(\mathrm{C}=\mathrm{O})}$ \\
\hline
\end{tabular}




\begin{tabular}{|c|c|c|}
\hline & & $\begin{array}{l}{ }_{(\mathrm{CHcycl})} \mathrm{C} 3-\mathrm{H} 3 \cdots \mathrm{O} 2_{(\mathrm{C}=\mathrm{O})} \&(\mathrm{CH} 2) \mathrm{C} 16-\mathrm{H} 13 \cdots \mathrm{O} 3_{(\mathrm{C}=\mathrm{O})} \\
\left.{ }_{*}^{*} \mathrm{CHcycl}\right) \\
\mathrm{C} 4-\mathrm{H} 4 \cdots \mathrm{O} 4_{(\mathrm{OH})} \&(\mathrm{CH} 2) \\
\mathrm{C} 16-\mathrm{H} 13 \cdots 3_{(\mathrm{C}=\mathrm{O})}\end{array}$ \\
\hline & $\mathrm{C}_{2}^{2}(18)$ & $\begin{array}{l}{ }_{(\mathrm{CHcycl})} \mathrm{C} 3-\mathrm{H} 3 \cdots \mathrm{O} 2_{(\mathrm{C}=\mathrm{O})} \&(\mathrm{CHcycl}) \mathrm{C} 11-\mathrm{H} 9 \cdots \mathrm{O} 5_{(\mathrm{NO} 2)} \\
{ }_{(\mathrm{CHcycl})} \mathrm{C} 11-\mathrm{H} 9 \cdots \mathrm{O} 5_{(\mathrm{NO} 2)} \&(\mathrm{CH} 2) \mathrm{C} 14-\mathrm{H} 12 \cdots \mathrm{O} 3_{(\mathrm{C}=\mathrm{O})}\end{array}$ \\
\hline & $\mathrm{C}_{2}^{2}(19)$ & 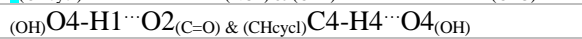 \\
\hline & $\mathrm{C}_{2}^{2}(20)$ & 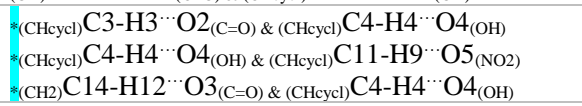 \\
\hline & $\mathrm{C}^{3}{ }_{4}(20)$ & ${ }_{(\mathrm{CH} 2)} \mathrm{C} 14-\mathrm{H} 12 \cdots \mathrm{O} 3_{(\mathrm{C}=\mathrm{O})} \&(\mathrm{CH} 2) \mathrm{C} 16-\mathrm{H} 13 \cdots \mathrm{O} 3_{(\mathrm{C}=\mathrm{O})}$ \\
\hline & $\mathrm{C}_{4}^{4}(20)$ & {$\left[(\mathrm{OH}) \mathrm{O} 4-\mathrm{H} 1 \cdots \mathrm{O} 2_{(\mathrm{C}=\mathrm{O})}\right] 2 \&\left[(\mathrm{CH} 2) \mathrm{C} 16-\mathrm{H} 13 \cdots \mathrm{O} 3_{(\mathrm{C}=\mathrm{O})}\right] 2$} \\
\hline & $\mathrm{R}_{2}^{2}(9)$ & ${ }_{(\mathrm{OH})} \mathrm{O} 4-\mathrm{H} 1 \cdots \mathrm{O} 2_{(\mathrm{C}=\mathrm{O})} \&(\mathrm{CH} 2) \mathrm{C} 14-\mathrm{H} 12 \cdots \mathrm{O} 3_{(\mathrm{C}=\mathrm{O})}$ \\
\hline & $\mathrm{R}_{4}^{3}(20)$ & ${ }_{(\mathrm{CH} 2)} \mathrm{C} 14-\mathrm{H} 12 \cdots \mathrm{O} 3_{(\mathrm{C}=\mathrm{O})} \&(\mathrm{CH} 2) \mathrm{C} 16-\mathrm{H} 13 \cdots \mathrm{O} 3_{(\mathrm{C}=\mathrm{O})}$ \\
\hline & $\mathrm{R}_{4}^{4}(20)$ & {$\left[(\mathrm{OH}) \mathrm{O} 4-\mathrm{H} 1 \cdots \mathrm{O} 2_{(\mathrm{C}=\mathrm{O})] 2} \&\left[(\mathrm{CH} 2) \mathrm{C} 16-\mathrm{H} 13 \cdots \mathrm{O} 3_{(\mathrm{C}=\mathrm{O})}\right] 2\right.$} \\
\hline VERXOI & $\mathrm{S}(7)$ & $(\mathrm{CHcycl}) \mathrm{C} 44-\mathrm{H} 38 \cdots \mathrm{O} 5_{(\mathrm{C}-\mathrm{O}-\mathrm{C})}$ \\
\hline & $\mathrm{C}(7)$ & $\begin{array}{l}{ }_{(\mathrm{OH})} \mathrm{O} 4-\mathrm{H} 1 \cdots \mathrm{O} 2_{(\mathrm{C}=\mathrm{O})} \\
{ }_{(\mathrm{OH})} \mathrm{O} 8-\mathrm{H} 22 \cdots 6_{(\mathrm{C}=\mathrm{O})}\end{array}$ \\
\hline & $\mathrm{C}(8)$ & $\begin{array}{l}{ }^{*}{ }_{(\mathrm{CH} 2)} \mathrm{C} 14-\mathrm{H} 11 \cdots \mathrm{O} 3_{(\mathrm{C}=\mathrm{O})} \\
{ }_{(\mathrm{CH} 2)} \mathrm{C} 38-\mathrm{H} 33 \cdots \mathrm{O} 7_{(\mathrm{C}=\mathrm{O})}\end{array}$ \\
\hline & $\mathrm{C}(12)$ & $(\mathrm{CHcycl}) \mathrm{C} 4-\mathrm{H} 4 \cdots \mathrm{O} 4_{(\mathrm{C}=\mathrm{O})}$ \\
\hline & $\mathrm{D}(2)$ & $\begin{array}{l}*{ }_{(\mathrm{CHcycl})} \mathrm{C} 3-\mathrm{H} 3 \cdots \mathrm{O}_{(\mathrm{C}=\mathrm{O})} \\
*_{(\mathrm{CHcycl})} \mathrm{C} 27-\mathrm{H} 24 \cdots \mathrm{O} 2_{(\mathrm{C}=\mathrm{O})} \\
{ }_{(\mathrm{CH} 2)} \mathrm{C} 16-\mathrm{H} 14 \cdots \mathrm{O} 7_{(\mathrm{C}=\mathrm{O})} \\
*_{(\mathrm{CH} 2)} \mathrm{C} 40-\mathrm{H} 34 \cdots \mathrm{O} 3_{(\mathrm{C}=\mathrm{O})}\end{array}$ \\
\hline Level 2 & $\mathrm{C}_{2}^{2}(8)$ & ${ }_{(\mathrm{CH} 2)} \mathrm{C} 16-\mathrm{H} 14 \cdots \mathrm{O} 7_{(\mathrm{C}=\mathrm{O})} \&(\mathrm{CH} 2) \mathrm{C} 40-\mathrm{H} 34 \cdots \mathrm{O} 3_{(\mathrm{C}=\mathrm{O})}$ \\
\hline & $\mathrm{C}_{2}^{2}(10)$ & ${ }_{(\mathrm{CHcycl})} \mathrm{C} 4-\mathrm{H} 4 \cdots \mathrm{O} 4_{(\mathrm{OH})} \&(\mathrm{CH} 2) \mathrm{C} 14-\mathrm{H} 11 \cdots \mathrm{O} 3_{(\mathrm{C}=\mathrm{O})}$ \\
\hline & $\mathrm{C}_{2}^{2}(11)$ & $(\mathrm{OH}) \mathrm{O} 4-\mathrm{H} 1 \cdots \mathrm{O} 2_{(\mathrm{C}=\mathrm{O})} \&(\mathrm{CHcycl}) \mathrm{C} 4-\mathrm{H} 4 \cdots \mathrm{O} 4_{(\mathrm{OH})}$ \\
\hline & $\mathrm{C}_{2}^{2}(15)$ & $\begin{array}{l}*(\mathrm{OH}) \mathrm{O} 4-\mathrm{H} 1 \cdots \mathrm{O} 2_{(\mathrm{C}=\mathrm{O})} \&(\mathrm{CH} 2) \mathrm{C} 14-\mathrm{H} 11 \cdots \mathrm{O} 3_{(\mathrm{C}=\mathrm{O})} \\
*(\mathrm{OH}) \mathrm{O} 8-\mathrm{H} 22 \cdots{ }^{\circ} 6_{(\mathrm{C}=\mathrm{O})} \&(\mathrm{CH} 2) \mathrm{C} 38-\mathrm{H} 33 \cdots \mathrm{O} 7_{(\mathrm{C}=\mathrm{O})}\end{array}$ \\
\hline & $\mathrm{C}_{2}^{2}(16)$ & $\begin{array}{l}{ }_{(\mathrm{CH})} \mathrm{C} 3-\mathrm{H} 3 \cdots 6_{(\mathrm{C}=\mathrm{O})} \&(\mathrm{CH} 2) \mathrm{C} 16-\mathrm{H} 14 \cdots \mathrm{O} 7_{(\mathrm{C}=\mathrm{O})} \\
{ }_{(\mathrm{CH})} \mathrm{C} 3-\mathrm{H} 3 \cdots 6_{(\mathrm{C}=\mathrm{O})} \&(\mathrm{CHcycl}) \mathrm{C} 27-\mathrm{H} 24 \cdots \mathrm{O} 2_{(\mathrm{C}=\mathrm{O})} \\
{ }_{(\mathrm{CH})} \mathrm{C} 3-\mathrm{H} 3 \cdots 6_{(\mathrm{C}=\mathrm{O})} \&(\mathrm{CH} 2) \mathrm{C} 40-\mathrm{H} 34 \cdots \mathrm{O} 3_{(\mathrm{C}=\mathrm{O})} \\
{ }_{(\mathrm{CH} 2)} \mathrm{C} 16-\mathrm{H} 14 \cdots \mathrm{O} 7_{(\mathrm{C}=\mathrm{O})} \&(\mathrm{CHcycl}) \\
{ }_{(\mathrm{CHcycl})} \mathrm{C} 27-\mathrm{H} 24 \cdots \mathrm{O} 2_{(\mathrm{C}=\mathrm{O})}-\mathrm{H} 24 \cdots \mathrm{O} 2_{(\mathrm{C}=\mathrm{O})} \&(\mathrm{CH} 2) \\
\mathrm{C} 40-\mathrm{H} 34 \cdots \mathrm{O} 3_{(\mathrm{C}=\mathrm{O})}\end{array}$ \\
\hline & $\mathrm{C}_{2}^{2}(19)$ & 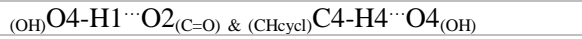 \\
\hline & $\mathrm{C}_{2}^{2}(20)$ & ${ }_{(\mathrm{CHcycl})} \mathrm{C} 4-\mathrm{H} 4 \cdots \mathrm{O} 4_{(\mathrm{OH})} \&(\mathrm{CH} 2) \mathrm{C} 14-\mathrm{H} 11 \cdots \mathrm{O} 3_{(\mathrm{C}=\mathrm{O})}$ \\
\hline & $\mathrm{R}_{2}^{2}(9)$ & $\begin{array}{l}*_{(\mathrm{OH})} \mathrm{O} 4-\mathrm{H} 1 \cdots \mathrm{O} 2_{(\mathrm{C}=\mathrm{O})} \&(\mathrm{CH} 2) \mathrm{C} 14-\mathrm{H} 11 \cdots \mathrm{O} 3_{(\mathrm{C}=\mathrm{O})} \\
*_{(\mathrm{OH})} \mathrm{O} 8-\mathrm{H} 22 \cdots 6_{(\mathrm{C}=\mathrm{O})} \&(\mathrm{CH} 2) \mathrm{C} 38-\mathrm{H} 33 \cdots \mathrm{O} 7_{(\mathrm{C}=\mathrm{O})}\end{array}$ \\
\hline & $\mathrm{D}_{3}^{2}(10)$ & $\begin{array}{l}{ }_{(\mathrm{OH})} \mathrm{O} 4-\mathrm{H} 1 \cdots \mathrm{O} 2_{(\mathrm{C}=\mathrm{O})} \&[(\mathrm{CHcycl}) \\
{ }_{(\mathrm{CH})} \mathrm{C} 3-\mathrm{H} 3 \cdots \mathrm{O} 6_{(\mathrm{C}=\mathrm{O})} \&(\mathrm{OH}) \mathrm{O} 8-\mathrm{H} 24 \cdots \mathrm{O} 222_{(\mathrm{C}=\mathrm{O}) 12} \mathrm{O} 6_{(\mathrm{C}=\mathrm{O})}\end{array}$ \\
\hline & $\mathrm{D}_{3}^{2}(11)$ & $\begin{array}{l}{ }_{(\mathrm{CH} 2)} \mathrm{C} 14-\mathrm{H} 11 \cdots \mathrm{O} 3_{(\mathrm{C}=\mathrm{O})} \&\left[{ }_{(\mathrm{CHcycl})} \mathrm{C} 27-\mathrm{H} 24 \cdots \mathrm{O} 2_{(\mathrm{C}=\mathrm{O})] 2}\right. \\
{ }_{(\mathrm{CH} 2)} \mathrm{C} 14-\mathrm{H} 11 \cdots \mathrm{O} 3_{(\mathrm{C}=\mathrm{O})} \&\left[(\mathrm{CH} 2) \mathrm{C} 40-\mathrm{H} 34 \cdots \mathrm{O} 3_{(\mathrm{C}=\mathrm{O})}\right]_{2} \\
\left.{ }_{[(\mathrm{CH} 2)} \mathrm{C} 16-\mathrm{H} 14 \cdots \mathrm{O} 7_{(\mathrm{C}=\mathrm{O})}\right] 2 \&(\mathrm{CH} 2) \mathrm{C} 38-\mathrm{H} 33^{\cdots} \mathrm{O} 7_{(\mathrm{C}=\mathrm{O})}\end{array}$ \\
\hline & $\mathrm{D}_{3}^{3}(12)$ & $\begin{array}{l}{ }_{(\mathrm{OH})} \mathrm{O} 4-\mathrm{H} 1 \cdots \mathrm{O} 2_{(\mathrm{C}=\mathrm{O})} \&\left[\left(\mathrm{CH}_{2}\right) \mathrm{C} 16-\mathrm{H} 14 \cdots \mathrm{O} 7_{(\mathrm{C}=\mathrm{O})]_{2}}\right. \\
{ }_{(\mathrm{OH})} \mathrm{O} 4-\mathrm{H} 1 \cdots \mathrm{O} 2_{(\mathrm{C}=\mathrm{O})} \&\left[(\mathrm{CH} 2) \mathrm{C} 40-\mathrm{H} 34 \cdots \mathrm{O} 3_{(\mathrm{C}=\mathrm{O})]_{2}}\right. \\
{ }_{(\mathrm{OH})} \mathrm{O} 8-\mathrm{H} 22 \cdots \mathrm{O} 6_{(\mathrm{C}=\mathrm{O})} \&\left[(\mathrm{CH} 2) \mathrm{C} 16-\mathrm{H} 14 \cdots \mathrm{O} 7_{(\mathrm{C}=\mathrm{O})]_{2}}\right. \\
*_{(\mathrm{OH})} \mathrm{O} 8-\mathrm{H} 22 \cdots \mathrm{O} 6_{(\mathrm{C}=\mathrm{O})} \&\left[\left({ }_{(\mathrm{CH} 2)} \mathrm{C} 40-\mathrm{H} 34 \cdots \mathrm{O} 3_{(\mathrm{C}=\mathrm{O})]_{2}}\right.\right.\end{array}$ \\
\hline & $\mathrm{D}_{3}^{3}(13)$ & $\begin{array}{l}\left.{ }_{[(\mathrm{CH})} \mathrm{C} 3-\mathrm{H} 3 \cdots \mathrm{O}_{(\mathrm{C}=\mathrm{O})}\right] 2 \&(\mathrm{CH} 2) \\
\mathrm{C} 38-\mathrm{H} 33 \cdots \mathrm{O} 7_{(\mathrm{C}=\mathrm{O})} \\
{ }_{(\mathrm{CHcycl})} \mathrm{C} 4-\mathrm{H} 4 \cdots \mathrm{O} 4_{(\mathrm{OH})} \&(\mathrm{CH} 2) \mathrm{C} 16-\mathrm{H} 14 \cdots \mathrm{O} 7_{(\mathrm{C}=\mathrm{O})} \\
{ }_{(\mathrm{CH} 2)} \mathrm{C} 14-\mathrm{H} 11 \cdots \mathrm{O} 3_{(\mathrm{C}=\mathrm{O})} \&\left[(\mathrm{CHcycl}) \mathrm{C} 27-\mathrm{H} 24 \cdots \mathrm{O} 2_{(\mathrm{C}=\mathrm{O})] 2}\right. \\
{ }_{(\mathrm{CH} 2)} \mathrm{C} 38-\mathrm{H} 33 \cdots \mathrm{O} 7_{(\mathrm{C}=\mathrm{O})} \&\left[(\mathrm{CH} 2) \mathrm{C} 40-\mathrm{H} 34 \cdots \mathrm{O} 3_{(\mathrm{C}=\mathrm{O})] 2}\right.\end{array}$ \\
\hline & $\mathrm{D}_{3}^{3}(16)$ & ${ }_{(\mathrm{CH} 2)} \mathrm{C} 40-\mathrm{H} 34 \cdots \mathrm{O} 3_{(\mathrm{C}=\mathrm{O})} \&\left[(\mathrm{CHcycl}) \mathrm{C} 27-\mathrm{H} 24 \cdots \mathrm{O} 2_{(\mathrm{C}=\mathrm{O})}\right] 2$ \\
\hline & $\mathrm{D}_{3}^{3}(17)$ & 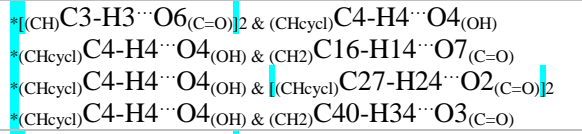 \\
\hline & $\mathrm{D}^{3}{ }_{3}(19)$ & 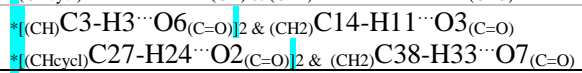 \\
\hline XAVYIE & $\mathrm{D}(2)$ & $\begin{array}{l}*_{(\mathrm{OH})} \mathrm{O} 1-\mathrm{H} 1 \cdots \mathrm{O} 7_{(\mathrm{OH})} \\
*_{(\mathrm{OH})} \mathrm{O} 7-\mathrm{H} 37 \cdots \mathrm{O} 5_{(\mathrm{C}=\mathrm{O})} \mathrm{C} 29-\mathrm{H} 40 \cdots \mathrm{O} 2_{(\mathrm{C}=\mathrm{O})}\end{array}$ \\
\hline & $\mathrm{C}(4)$ & ${ }_{(\mathrm{NH})} \mathrm{N} 2-\mathrm{H} 16 \cdots \mathrm{O} 3_{(\mathrm{C}=\mathrm{O})}$ \\
\hline & $\mathrm{R}_{2}^{2}(8)$ & {$\left[(\mathrm{CH} 2) \mathrm{C} 2-\mathrm{H} 2 \cdots \mathrm{O} 1_{(\mathrm{OH})}\right] 2$} \\
\hline Level 2 & $\mathrm{C}_{2}^{1}(11)$ & ${ }_{(\mathrm{CH} 2)} \mathrm{C} 2-\mathrm{H} 2 \cdots \mathrm{O} 1_{(\mathrm{OH})} \&\left(\mathrm{CHCycl}_{1}\right) \mathrm{C} 27-\mathrm{H} 36 \cdots \mathrm{O} 1_{(\mathrm{OH})}$ \\
\hline & $\mathrm{C}^{2}{ }_{2}(9)$ & ${ }_{(\mathrm{OH})} \mathrm{O} 1-\mathrm{H} 1 \cdots \mathrm{O} 7_{(\mathrm{OH})} \&(\mathrm{OH}) \mathrm{O} 7-\mathrm{H} 37 \cdots \mathrm{O} 5_{(\mathrm{C}=\mathrm{O})}$ \\
\hline & $\mathrm{C}_{2}^{2}(15)$ & ${ }_{(\mathrm{CH} 2)} \mathrm{C} 2-\mathrm{H} 2 \cdots \mathrm{O} 1_{(\mathrm{OH})} \&(\mathrm{CHcycl}) \mathrm{C} 27-\mathrm{H} 36 \cdots \mathrm{O} 1_{(\mathrm{OH})}$ \\
\hline & $\mathrm{R}_{2}^{2}(14)$ & 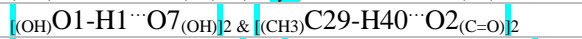 \\
\hline & $\mathrm{R}_{4}^{4}(20)$ & {$\left[(\mathrm{OH}) \mathrm{O} 7-\mathrm{H} 37 \cdots \mathrm{O} 5_{(\mathrm{C}=\mathrm{O})}\right] 2 \&\left[(\mathrm{CH} 3) \mathrm{C} 29-\mathrm{H} 40 \cdots \mathrm{O} 2_{(\mathrm{C}=\mathrm{O})}\right]_{2}$} \\
\hline & $\mathrm{D}_{3}^{3}(9)$ & $\begin{array}{l}*(\mathrm{OH}) \mathrm{O} 1-\mathrm{H} 1 \cdots \mathrm{O} 7_{(\mathrm{OH}) \mid 2} \&(\mathrm{CH} 2) \mathrm{C} 2-\mathrm{H} 2 \cdots \mathrm{O} 1_{(\mathrm{OH})} \\
{ }_{(\mathrm{CH} 2)} \mathrm{C} 2-\mathrm{H} 2 \cdots \mathrm{O} 1_{(\mathrm{OH})} \&\left[(\mathrm{CH} 3) \mathrm{C} 29-\mathrm{H} 40 \cdots \mathrm{O} 2_{(\mathrm{C}=\mathrm{O})]_{2}}\right.\end{array}$ \\
\hline & $\mathrm{D}_{3}^{3}(13)$ & {$\left[(\mathrm{OH}) \mathrm{O} 7-\mathrm{H} 37 \cdots \mathrm{O} 5_{(\mathrm{C}=\mathrm{O})}\right]_{2} \&(\mathrm{CH} 2) \mathrm{C} 2-\mathrm{H} 2 \cdots \mathrm{O} 1_{(\mathrm{OH})}$} \\
\hline & $\mathrm{D}_{3}^{3}(16)$ & 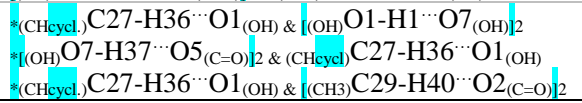 \\
\hline
\end{tabular}

Fmoc -CYSTEINE derivatives 


\begin{tabular}{|c|c|c|}
\hline \multirow[t]{4}{*}{ QOFHID } & $\mathrm{S}(11)$ & ${ }_{\text {(CHcyl.) }} \mathrm{C} 11-\mathrm{H} 16 \cdots \mathrm{O} 2_{(\mathrm{C}=\mathrm{O})}$ \\
\hline & $\mathrm{C}(4)$ & $(\mathrm{OH}) \mathrm{O} 1-\mathrm{H} 1 \cdots \mathrm{O} 2_{(\mathrm{C}=\mathrm{O})}$ \\
\hline & $\mathrm{C}(5)$ & $\begin{array}{l}{ }_{(\mathrm{NH})} \mathrm{N} 1-\mathrm{H} 2 \cdots \mathrm{O} 1_{(\mathrm{OH})} \\
{ }_{(\mathrm{CH})} \mathrm{C} 1-\mathrm{H} 3 \cdots \mathrm{O} 4_{(\mathrm{C}-\mathrm{O}-\mathrm{C})}\end{array}$ \\
\hline & $\mathrm{C}(19)$ & $\begin{array}{l}\text { (CHcycl.) } \mathrm{C} 14-\mathrm{H} 19 \cdots \mathrm{O} 3_{(\mathrm{C}=\mathrm{O})} \\
(\mathrm{CHcycl.}) \\
\mathrm{C} 17-\mathrm{H} 20 \cdots \mathrm{O} 3_{(\mathrm{C}=\mathrm{O})}\end{array}$ \\
\hline \multirow[t]{15}{*}{ Level 2} & $\mathrm{C}_{2}^{2}(7)$ & 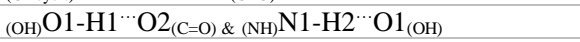 \\
\hline & $\mathrm{C}_{2}^{2}(9)$ & $(\mathrm{OH}) \mathrm{O} 1-\mathrm{H} 1 \cdots \mathrm{O} 2_{(\mathrm{C}=\mathrm{O})} \&(\mathrm{NH}) \mathrm{N} 1-\mathrm{H} 2 \cdots \mathrm{O} 1_{(\mathrm{OH})}$ \\
\hline & $\mathrm{C}_{2}^{2}(10)$ & $(\mathrm{NH}) \mathrm{N} 1-\mathrm{H} 2 \cdots \mathrm{O} 1_{(\mathrm{OH})} \&(\mathrm{CH}) \mathrm{C} 1-\mathrm{H} 3 \cdots \mathrm{O} 4_{(\mathrm{C}-\mathrm{O}-\mathrm{C})}$ \\
\hline & $\mathrm{C}_{2}^{2}(11)$ & ${ }_{(\mathrm{OH})} \mathrm{O} 1-\mathrm{H} 1 \cdots \mathrm{O} 2_{(\mathrm{C}=\mathrm{O})} \&(\mathrm{CH}) \mathrm{C} 1-\mathrm{H} 3 \cdots \mathrm{O} 4_{(\mathrm{C}-\mathrm{O}-\mathrm{C})}$ \\
\hline & $\mathrm{C}_{2}^{2}(12)$ & 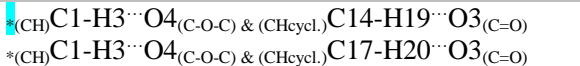 \\
\hline & $\mathrm{C}_{2}^{2}(14)$ & 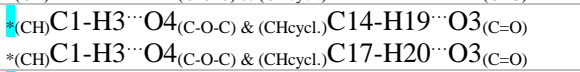 \\
\hline & $\mathrm{C}_{2}^{2}(16)$ & $\begin{array}{l}{ }_{(\mathrm{NH})} \mathrm{N} 1-\mathrm{H} 2 \cdots \mathrm{O} 1_{(\mathrm{OH})} \&(\mathrm{CHcycl} .) \mathrm{C} 14-\mathrm{H} 19 \cdots \mathrm{O} 3_{(\mathrm{C}=\mathrm{O})} \\
{ }_{(\mathrm{NH})} \mathrm{N} 1-\mathrm{H} 2 \cdots \mathrm{O} 1_{(\mathrm{OH})} \&(\mathrm{CHcyl} .) \\
\mathrm{C} 17-\mathrm{H} 20 \cdots \mathrm{O} 3_{(\mathrm{C}=\mathrm{O})}\end{array}$ \\
\hline & $\mathrm{C}_{2}^{2}(18)$ & $\left({ }_{(\mathrm{CH} \text { cycl. }} \mathrm{C} 14-\mathrm{H} 19 \cdots \mathrm{O} 3_{(\mathrm{C}=\mathrm{O})} \&(\mathrm{CHcycl}) \mathrm{C} 17-\mathrm{H} 20 \cdots \mathrm{O} 3_{(\mathrm{C}=\mathrm{O})}\right.$ \\
\hline & $\mathrm{C}_{2}^{2}(19)$ & $\begin{array}{l}{ }_{(\mathrm{OH})} \mathrm{O} 1-\mathrm{H} 1 \cdots \mathrm{O} 2_{(\mathrm{C}=\mathrm{O})} \&(\mathrm{CHcycl.}) \mathrm{C} 14-\mathrm{H} 19 \cdots \mathrm{O} 3_{(\mathrm{C}=\mathrm{O})} \\
{ }_{(\mathrm{OH})} \mathrm{O} 1-\mathrm{H} 1 \cdots \mathrm{O} 2_{(\mathrm{C}=\mathrm{O})} \&(\mathrm{CHcycl.}) \\
\mathrm{C} 17-\mathrm{H} 20 \cdots \mathrm{O} 3_{(\mathrm{C}=\mathrm{O})}\end{array}$ \\
\hline & $\mathrm{R}_{2}^{1}(7)$ & [(CHcycl. $) \mathrm{C} 17-\mathrm{H} 20 \cdots \mathrm{O} 3_{(\mathrm{C}=0)]_{2}}$ \\
\hline & $\mathrm{R}_{2}^{2}(8)$ & ${ }_{(\mathrm{NH})} \mathrm{N} 1-\mathrm{H} 2 \cdots \mathrm{O} 1_{(\mathrm{OH})} \&(\mathrm{CH}) \mathrm{C} 1-\mathrm{H} 3 \cdots \mathrm{O} 4_{(\mathrm{C}-\mathrm{O}-\mathrm{C})}$ \\
\hline & $\mathrm{R}_{3}^{3}(11)$ & {$\left[(\mathrm{OH}) \mathrm{O} 1-\mathrm{H} 1 \cdots \mathrm{O} 2_{(\mathrm{C}=\mathrm{O})} \mid 2 \&(\mathrm{NH}) \mathrm{N} 1-\mathrm{H} 2 \cdots \mathrm{O} 1_{(\mathrm{OH})}\right.$} \\
\hline & $\mathrm{R}_{3}^{3}(15)$ & {$\left[(\mathrm{OH}) \mathrm{O} 1-\mathrm{H} 1 \cdots \mathrm{O} 2_{(\mathrm{C}=\mathrm{O})}\right] 2 \&(\mathrm{CH}) \mathrm{C} 1-\mathrm{H} 3 \cdots \mathrm{O} 4_{(\mathrm{C}-\mathrm{O}-\mathrm{C})}$} \\
\hline & $\mathrm{R}_{4}^{4}(16)$ & 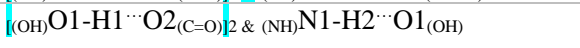 \\
\hline & & Fmoc-LEUCINE derivatives \\
\hline \multirow[t]{3}{*}{ BIZXUE } & $\mathrm{C}(5)$ & $\begin{array}{l}{ }_{(\mathrm{NH})} \mathrm{N} 1-\mathrm{H} 2 \cdots \mathrm{O} 1_{(\mathrm{C}=\mathrm{O})} \\
{ }_{(\mathrm{CH})} \mathrm{C} 1-\mathrm{H} 3 \cdots \mathrm{O} 4{ }_{(\mathrm{C}-\mathrm{O}-\mathrm{C})} \\
{ }_{(\mathrm{CH} 2)} \mathrm{C} 8-\mathrm{H} 14 \cdots \mathrm{O} 3_{(\mathrm{C}=\mathrm{O})}\end{array}$ \\
\hline & $C(7)$ & ${ }_{(\mathrm{OH})} \mathrm{O} 2-\mathrm{H} 1 \cdots \mathrm{O} 3_{(\mathrm{C}=\mathrm{O})}$ \\
\hline & $\mathrm{C}(8)$ & ${ }_{(\mathrm{CH} 2)} \mathrm{C} 8-\mathrm{H} 13 \cdots \mathrm{O} 1_{(\mathrm{C}=\mathrm{O})}$ \\
\hline \multirow[t]{16}{*}{ Level 2} & $\mathrm{C}_{2}^{1}(7)$ & ${ }_{(\mathrm{NH})} \mathrm{N} 1-\mathrm{H} 2 \cdots \mathrm{O} 1_{(\mathrm{C}=\mathrm{O})} \&(\mathrm{CH} 2) \mathrm{C} 8-\mathrm{H} 13 \cdots \mathrm{O} 1_{(\mathrm{C}=\mathrm{O})}$ \\
\hline & $\mathrm{C}_{2}^{1}(10)$ & ${ }_{(\mathrm{CH} 2)} \mathrm{C} 8-\mathrm{H} 14 \cdots \mathrm{O} 3_{(\mathrm{C}=\mathrm{O})} \&(\mathrm{OH}) \mathrm{O} 2-\mathrm{H} 1 \cdots \mathrm{O} 3_{(\mathrm{C}=\mathrm{O})}$ \\
\hline & $\mathrm{C}_{2}^{2}(7)$ & ${ }_{(\mathrm{CH})} \mathrm{C} 1-\mathrm{H} 3 \cdots \mathrm{O} 4_{(\mathrm{C}-\mathrm{O}-\mathrm{C})} \&{ }_{(\mathrm{CH} 2)} \mathrm{C} 8-\mathrm{H} 13 \cdots \mathrm{O} 1_{(\mathrm{C}=\mathrm{O})}$ \\
\hline & $\mathrm{C}_{2}^{2}(8)$ & $\begin{array}{l}{ }^{(\mathrm{OH})} \mathrm{O} 2-\mathrm{H} 1 \cdots \mathrm{O} 3_{(\mathrm{C}=\mathrm{O})} \&(\mathrm{NH}) \\
\\
{ }_{(\mathrm{OH})} \mathrm{O} 1-\mathrm{O} 2-\mathrm{H} 1 \cdots{ }^{2} \cdots 3_{(\mathrm{C}=\mathrm{O})} \&(\mathrm{CH}) \\
\mathrm{C} 1-\mathrm{H} 3 \cdots \mathrm{O} 4_{(\mathrm{C}-\mathrm{O}-\mathrm{C})}\end{array}$ \\
\hline & $\mathrm{C}_{2}^{2}(9)$ & ${ }_{(\mathrm{CH} 2)} \mathrm{C} 8-\mathrm{H} 13 \cdots \mathrm{O} 1_{(\mathrm{C}=\mathrm{O})} \&(\mathrm{CH} 2) \mathrm{C} 8-\mathrm{H} 14 \cdots \mathrm{O} 3_{(\mathrm{C}=\mathrm{O})}$ \\
\hline & $\mathrm{C}_{2}^{2}(10)$ & $\begin{array}{l}{ }_{(\mathrm{NH})} \mathrm{N} 1-\mathrm{H} 2 \cdots \mathrm{O} 1_{(\mathrm{C}=\mathrm{O})} \&(\mathrm{CH}) \mathrm{C} 1-\mathrm{H} 3 \cdots \mathrm{O} 4_{(\mathrm{C}-\mathrm{O}-\mathrm{C})} \\
{ }_{(\mathrm{CH})} \mathrm{C} 1-\mathrm{H} 3 \cdots \mathrm{O} 4_{(\mathrm{C}-\mathrm{O}-\mathrm{C})} \&(\mathrm{CH} 2) \\
\mathrm{C} 8-\mathrm{H} 14 \cdots{ }^{2} 3_{(\mathrm{C}=\mathrm{O})}\end{array}$ \\
\hline & $\mathrm{C}_{2}^{2}(12)$ & $\begin{array}{l}{ }^{(\mathrm{OH})} \mathrm{O} 2-\mathrm{H} 1 \cdots \mathrm{O} 3_{(\mathrm{C}=\mathrm{O})} \&(\mathrm{NH}) \mathrm{N} 1-\mathrm{H} 2 \cdots \mathrm{O} 1_{(\mathrm{C}=\mathrm{O})} \\
{ }^{(\mathrm{OH})} \mathrm{O} 2-\mathrm{H} 1 \cdots \mathrm{O} 3_{(\mathrm{C}=\mathrm{O})} \&(\mathrm{CH}) \mathrm{C} 1-\mathrm{H} 3 \cdots \mathrm{O} 4_{(\mathrm{C}-\mathrm{O}-\mathrm{C})} \\
{ }^{(\mathrm{OH})} \mathrm{O} 2-\mathrm{H} 1 \cdots \mathrm{O} 3_{(\mathrm{C}=\mathrm{O})} \&(\mathrm{CH} 2) \mathrm{C} 8-\mathrm{H} 14 \cdots \mathrm{O} 3_{(\mathrm{C}=\mathrm{O})} \\
{ }_{(\mathrm{NH})} \mathrm{N} 1-\mathrm{H} 2 \cdots \mathrm{O} 1_{(\mathrm{C}=\mathrm{O})} \&(\mathrm{CH} 2) \\
\mathrm{C} 8-\mathrm{H} 14 \cdots \mathrm{O} 3_{(\mathrm{C}=\mathrm{O})}\end{array}$ \\
\hline & $\mathrm{C}_{2}^{2}(13)$ & $\begin{array}{l}{ }^{*}{ }_{(\mathrm{NH})} \mathrm{N} 1-\mathrm{H} 2 \cdots \mathrm{O} 1_{(\mathrm{C}=\mathrm{O})} \&(\mathrm{CH} 2) \mathrm{C} 8-\mathrm{H} 13 \cdots \mathrm{O} 1_{(\mathrm{C}=\mathrm{O})} \\
{ }^{(\mathrm{CH})} \mathrm{C} 1-\mathrm{H} 3 \cdots \mathrm{O} 4_{(\mathrm{C}-\mathrm{O}-\mathrm{C})} \&(\mathrm{CH} 2) \mathrm{C} 8-\mathrm{H} 13 \cdots \mathrm{O} 1_{(\mathrm{C}=\mathrm{O})} \\
{ }_{(\mathrm{CH} 2)} \mathrm{C} 8-\mathrm{H} 13 \cdots 1_{(\mathrm{C}=\mathrm{O})} \&(\mathrm{CH} 2) \\
\mathrm{C} 8-\mathrm{H} 14 \cdots \mathrm{O} 3_{(\mathrm{C}=\mathrm{O})}\end{array}$ \\
\hline & $\mathrm{C}_{2}^{2}(15)$ & $(\mathrm{OH}) \mathrm{O} 2-\mathrm{H} 1 \cdots \mathrm{O} 3_{(\mathrm{C}=\mathrm{O})} \&(\mathrm{CH} 2) \mathrm{C} 8-\mathrm{H} 13 \cdots \mathrm{O} 1_{(\mathrm{C}=\mathrm{O})}$ \\
\hline & $\mathrm{C}_{4}^{3}(20)$ & $(\mathrm{NH})=1-\mathrm{H} 2 \cdots \mathrm{O} 1_{(\mathrm{C}=\mathrm{O})} \&(\mathrm{CH} 2)$ \\
\hline & $\mathrm{C}_{4}^{4}(20)$ & 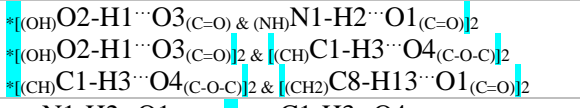 \\
\hline & $\mathrm{R}_{2}^{2}(8)$ & $\begin{array}{l}(\mathrm{NH}) \mathrm{N} 1-\mathrm{H} 2 \cdots \mathrm{O} 1_{(\mathrm{C}=\mathrm{O})} \&(\mathrm{CH}) \mathrm{C} 1-\mathrm{H} 3 \cdots \mathrm{O} 4_{(\mathrm{C}-\mathrm{O}-\mathrm{C})} \\
(\mathrm{CH}) \mathrm{C} 1-\mathrm{H} 3 \cdots \mathrm{O} 4_{(\mathrm{C}-\mathrm{O}-\mathrm{C})} \&(\mathrm{CH} 2) \mathrm{C}-\mathrm{H} 14 \cdots \mathrm{O} 3_{(\mathrm{C}=\mathrm{O})}\end{array}$ \\
\hline & $\mathrm{R}_{2}^{2}(9)$ & $(\mathrm{OH}) \mathrm{O} 2-\mathrm{H} 1 \cdots \mathrm{O} 3_{(\mathrm{C}=\mathrm{O})} \&(\mathrm{CH} 2) \mathrm{C} 8-\mathrm{H} 13 \cdots \mathrm{O} 1_{(\mathrm{C}=\mathrm{O})}$ \\
\hline & $\mathrm{R}_{2}^{2}(12)$ & $(\mathrm{NH}) \mathrm{N} 1-\mathrm{H} 2 \cdots \mathrm{O} 1_{(\mathrm{C}=\mathrm{O})} \&(\mathrm{CH} 2) \mathrm{C} 8-\mathrm{H} 14 \cdots \mathrm{O} 3_{(\mathrm{C}=\mathrm{O})}$ \\
\hline & $\mathrm{R}_{4}^{3}(20)$ & {$\left[(\mathrm{NH}) \mathrm{N} 1-\mathrm{H} 2 \cdots \mathrm{O} 1_{(\mathrm{C}=\mathrm{O})} \mid 2 \&\left[(\mathrm{CH} 2) \mathrm{C} 8-\mathrm{H} 13 \cdots \mathrm{O} 1_{\left.(\mathrm{C}=\mathrm{O})\right|_{2}}\right.\right.$} \\
\hline & $\mathrm{R}_{4}^{4}(20)$ & 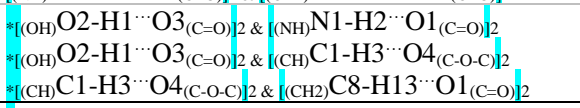 \\
\hline \multirow[t]{3}{*}{ EXOFAY } & $\mathrm{D}(2)$ & $\begin{array}{l}{ }_{(\mathrm{OH})} \mathrm{O} 1-\mathrm{H} 2 \cdots \mathrm{Cl} 1 \\
{ }_{(\mathrm{NH} 3+)} \mathrm{N} 1-\mathrm{H} 9 \cdots \mathrm{Cl} 1 \\
{ }_{(\mathrm{NH} 3+)} \mathrm{N} 1-\mathrm{H} 9 \cdots \mathrm{O} 5_{(\mathrm{C}-\mathrm{O}-\mathrm{C})} \\
{ }_{(\mathrm{NH}+3} \mathrm{N} 1-\mathrm{H} 11 \cdots \mathrm{Cl} 1\end{array}$ \\
\hline & $\mathrm{C}(4)$ & $(\mathrm{NH}) \mathrm{N} 2-\mathrm{H} 12 \cdots \mathrm{O} 4_{(\mathrm{C}=\mathrm{O})}$ \\
\hline & $\mathrm{C}(5)$ & $*_{(\mathrm{CH} 2)} \mathrm{C} 3-\mathrm{H} 4 \cdots \mathrm{O} 1_{(\mathrm{OH})}$ \\
\hline \multirow[t]{9}{*}{ level 2} & $\mathrm{C}_{2}^{1}(4)$ & $\left({ }_{(\mathrm{NH} 3+}^{+}\right) \mathrm{N} 1-\mathrm{H} 9 \cdots \mathrm{Cl} 1_{\&(\mathrm{NH} 3+)} \mathrm{N} 1-\mathrm{H} 11 \cdots \mathrm{Cl} 1$ \\
\hline & $\mathrm{C}_{2}^{1}(10)$ & ${ }_{(\mathrm{OH})} \mathrm{O} 1-\mathrm{H} 2 \cdots \mathrm{Cl} 1_{\&(\mathrm{NH} 3+)} \mathrm{N} 1-\mathrm{H} 9 \cdots \mathrm{Cl} 1$ \\
\hline & $\mathrm{C}_{2}^{2}(11)$ & ${ }_{(\mathrm{NH})} \mathrm{N} 2-\mathrm{H} 12 \cdots \mathrm{O} 4_{(\mathrm{C}=\mathrm{O})} \&(\mathrm{CH} 2) \mathrm{C} 3-\mathrm{H} 4 \cdots \mathrm{O} 1_{(\mathrm{OH})}$ \\
\hline & $\mathrm{R}_{2}^{2}(11)$ & 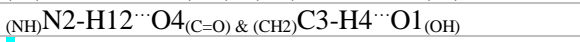 \\
\hline & $\mathrm{D}_{2}^{2}(5)$ & $\begin{array}{l}*_{(\mathrm{NH} 3++} \mathrm{N} 1-\mathrm{H} 9 \cdots \mathrm{Cl} 11_{\&(\mathrm{NH} 3++} \mathrm{N} 1-\mathrm{H} 10 \cdots \mathrm{O} 5_{(\mathrm{C}-\mathrm{O}-\mathrm{C})} \\
{ }_{\left({ }_{(\mathrm{NH} 3+}\right)} \mathrm{N} 1-\mathrm{H} 10 \cdots 5_{(\mathrm{C}-\mathrm{O}-\mathrm{C})} \&(\mathrm{NH} 3++) \\
\mathrm{N} 1-\mathrm{H} 11 \cdots \mathrm{Cl} 1\end{array}$ \\
\hline & $\mathrm{D}_{2}^{2}(11)$ & $(\mathrm{OH}) \mathrm{O} 1-\mathrm{H} 2 \cdots \mathrm{Cl} 1_{\&(\mathrm{NH} 3+)} \mathrm{N} 1-\mathrm{H} 10 \cdots \mathrm{O} 5_{(\mathrm{C}-\mathrm{O}-\mathrm{C})}$ \\
\hline & $\mathrm{D}_{3}^{3}(10)$ & {$\left[(\mathrm{OH}) \mathrm{O} 1-\mathrm{H} 2 \cdots \mathrm{Cl} 1_{2} \&\left(_{(\mathrm{CH} 2)} \mathrm{C} 3-\mathrm{H} 4 \cdots \mathrm{O} 1_{(\mathrm{OH})}\right.\right.$} \\
\hline & $\mathrm{D}_{3}^{3}(15)$ & ${ }_{(\mathrm{OH})} \mathrm{O} 1-\mathrm{H} 2 \cdots \mathrm{Cl} 1_{2 \&(\mathrm{NH})} \mathrm{N} 2-\mathrm{H} 14 \cdots \mathrm{O} 4_{(\mathrm{C}=\mathrm{O})}$ \\
\hline & $\mathrm{D}_{3}^{3}(16)$ & 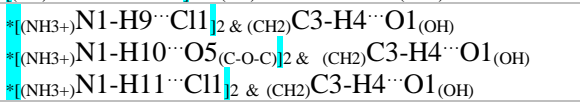 \\
\hline
\end{tabular}


$\mathrm{D}_{3}^{3}{ }_{3}(19) \quad *\left(\mathrm{NH}_{3}+\mathrm{N} 1-\mathrm{H} 9 \cdots \mathrm{Cl} 1_{2 \&(\mathrm{NH})} \mathrm{N} 2-\mathrm{H} 14 \cdots \mathrm{O} 4_{(\mathrm{C}=\mathrm{O})}\right.$

$*\left(\mathrm{NH}_{3}+\mathrm{N} 1-\mathrm{H} 10 \cdots \mathrm{O} 5_{(\mathrm{C}-\mathrm{O}-\mathrm{C})} 2\right.$ \& $(\mathrm{NH}) \mathrm{N} 2-\mathrm{H} 12 \cdots \mathrm{O} 4_{(\mathrm{C}=\mathrm{O})}$

${ }_{(\mathrm{NH} 3+)} \mathrm{N} 1-\mathrm{H} 11 \cdots \mathrm{Cl} 1_{\&}(\mathrm{NH}) \mathrm{N} 2-\mathrm{H} 12 \cdots \mathrm{O} 4_{(\mathrm{C}=\mathrm{O})}$

Fmoc SERINE derivatives

\begin{tabular}{|c|c|c|}
\hline ADAGOE & $\mathrm{D}(2)$ & $\begin{array}{l}*_{(\mathrm{Ccycl} .)} \mathrm{C} 1-\mathrm{H} 3 \cdots \mathrm{O}_{(\mathrm{H} 2 \mathrm{O})} \\
{ }_{(\mathrm{H} 2 \mathrm{O})} \mathrm{O} 6-\mathrm{H} 16 \cdots \mathrm{O} 4_{(\mathrm{C}=\mathrm{O})} \\
*_{(\mathrm{H} 2 \mathrm{O})} \mathrm{O} 6-\mathrm{H} 17 \cdots \mathrm{O} 2_{(\mathrm{C}=\mathrm{O})}\end{array}$ \\
\hline & $\mathrm{C}(5)$ & ${ }_{(\mathrm{NH})} \mathrm{N} 1-\mathrm{H} 2 \cdots \mathrm{O} 4_{(\mathrm{C}=\mathrm{O})}$ \\
\hline & $\mathrm{C}(9)$ & $($ Ccycl. $) \mathrm{C} 2-\mathrm{H} 4 \cdots \mathrm{O} 2_{(\mathrm{C}=\mathrm{O})}$ \\
\hline \multirow[t]{8}{*}{ Level 2} & $\mathrm{C}^{2}{ }_{2}(9)$ & ${ }_{(\mathrm{H} 2 \mathrm{O})} \mathrm{O} 6-\mathrm{H} 16 \cdots \mathrm{O} 4_{(\mathrm{C}=\mathrm{O})} \&(\mathrm{H} 2 \mathrm{O}) \mathrm{O} 6-\mathrm{H} 17 \cdots \mathrm{O} 2_{(\mathrm{C}=\mathrm{O})}$ \\
\hline & $\mathrm{C}_{2(10)}^{2}$ & ${ }_{(\mathrm{H} 2 \mathrm{O})} \mathrm{O} 6-\mathrm{H} 16 \cdots \mathrm{O} 4_{(\mathrm{C}=\mathrm{O})} \&\left(\mathrm{Ccycl}_{.}\right) \mathrm{C} 1-\mathrm{H} 3 \cdots \mathrm{O}_{(\mathrm{H} 20)}$ \\
\hline & $\mathrm{C}_{2}^{2}(13)$ & ${ }_{(\mathrm{Ccycl} .)} \mathrm{C} 1-\mathrm{H} 3 \cdots \mathrm{O} 6_{(\mathrm{H} 20)} \&(\mathrm{H} 2 \mathrm{O}) \mathrm{O} 6-\mathrm{H} 16 \cdots \mathrm{O} 4_{(\mathrm{C}=\mathrm{O})}$ \\
\hline & $\mathrm{C}_{2}^{2}(16)$ & 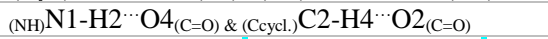 \\
\hline & $\mathrm{D}_{3}^{2}(8)$ & ${ }_{(\mathrm{NH})} \mathrm{N} 1-\mathrm{H} 2 \cdots \mathrm{O} 4_{(\mathrm{C}=\mathrm{O})} \&\left[(\mathrm{H} 2 \mathrm{O}) \mathrm{O} 6-\mathrm{H} 16 \cdots \mathrm{O} 4_{(\mathrm{C}=\mathrm{O})}\right]_{2}$ \\
\hline & $\mathrm{D}_{3}^{3}(12)$ & $\begin{array}{l}{ }_{(\mathrm{H} 2 \mathrm{O})} \mathrm{O} 6-\mathrm{H} 16 \cdots \mathrm{O} 4_{(\mathrm{C}=\mathrm{O})} \&(\mathrm{NH}) \mathrm{N} 1-\mathrm{H} 2 \cdots \mathrm{O} 4_{(\mathrm{C}=\mathrm{O})} \\
{ }_{(\mathrm{H} 2 \mathrm{O})} \mathrm{O} 6-\mathrm{H} 16 \cdots \mathrm{O} 4_{(\mathrm{C}=\mathrm{O})} \&(\mathrm{Ccycl} .) \\
\mathrm{C} 2-\mathrm{H} 4 \cdots \mathrm{O} 2_{(\mathrm{C}=\mathrm{O})}\end{array}$ \\
\hline & $\mathrm{D}_{3}^{3}(14)$ & 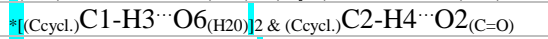 \\
\hline & $\mathrm{D}_{3}^{3}(20)$ & $*\left[(\mathrm{H} 2 \mathrm{O}) \mathrm{O} 6-\mathrm{H} 16 \cdots \mathrm{O} 4_{(\mathrm{C}=\mathrm{O})] 2} \&(\mathrm{Ccycl}) \mathrm{C} 2-\mathrm{H} 4 \cdots \mathrm{O} 2_{(\mathrm{C}=\mathrm{O})}\right.$ \\
\hline \multirow[t]{3}{*}{ MOHCIW } & $\mathrm{C}(5)$ & $(\mathrm{CH}) \mathrm{C} 1-\mathrm{H} 3 \cdots \mathrm{O} 5_{(\mathrm{C}-\mathrm{O}-\mathrm{C})}$ \\
\hline & $\mathrm{C}(7)$ & ${ }_{(\mathrm{OH})} \mathrm{O} 2-\mathrm{H} 1 \cdots \mathrm{O} 4_{(\mathrm{C}=\mathrm{O})}$ \\
\hline & $\mathrm{C}(8)$ & $\begin{array}{l}*(\mathrm{CH} 2) \mathrm{C} 5-\mathrm{H} 7 \cdots{ }^{\cdots} 1_{(\mathrm{C}=\mathrm{O})} \\
*(\mathrm{CHcycl}) \\
\mathrm{C} 8-\mathrm{H} 9 \cdots 4_{(\mathrm{C}=\mathrm{O})}\end{array}$ \\
\hline \multirow[t]{12}{*}{ Level 2} & $\mathrm{C}_{2}^{1}(13)$ & $(\mathrm{OH}) \mathrm{O} 2-\mathrm{H} 1 \cdots \mathrm{O} 4_{(\mathrm{C}=\mathrm{O})} \&(\mathrm{CHcycl}) \mathrm{C} 8-\mathrm{H} 9 \cdots \mathrm{O} 4_{(\mathrm{C}=\mathrm{O})}$ \\
\hline & $\mathrm{C}_{2(7)}^{2}$ & ${ }_{(\mathrm{CH})} \mathrm{C} 1-\mathrm{H} 3 \cdots \mathrm{O} 5_{(\mathrm{C}-\mathrm{O}-\mathrm{C})} \&(\mathrm{CH} 2) \mathrm{C} 5-\mathrm{H} 7 \cdots \mathrm{O} 1_{(\mathrm{C}=\mathrm{O})}$ \\
\hline & $\mathrm{C}_{2}^{2}(8)$ & ${ }_{(\mathrm{CH})} \mathrm{C} 1-\mathrm{H} 3 \cdots \mathrm{O} 5_{(\mathrm{C}-\mathrm{O}-\mathrm{C})} \&(\mathrm{OH}) \mathrm{O} 2-\mathrm{H} 1 \cdots \mathrm{O} 4_{(\mathrm{C}=\mathrm{O})}$ \\
\hline & $\mathrm{C}_{2}^{2}(9)$ & ${ }_{(\mathrm{OH})} \mathrm{O} 2-\mathrm{H} 1^{\cdots} \mathrm{O} 4_{(\mathrm{C}=\mathrm{O})} \&(\mathrm{CH} 2) \mathrm{C} 5-\mathrm{H} 7 \cdots \mathrm{O} 1_{(\mathrm{C}=\mathrm{O})}$ \\
\hline & $\mathrm{C}_{2}^{2}(12)$ & 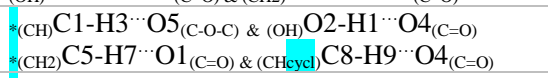 \\
\hline & $\mathrm{C}_{2}^{2}(13)$ & $\begin{array}{l}{ }_{(\mathrm{CH})} \mathrm{C} 1-\mathrm{H} 3 \cdots 5_{(\mathrm{C}-\mathrm{O}-\mathrm{C})} \&(\mathrm{CH}) \mathrm{C} 5-\mathrm{H} 7 \cdots \mathrm{O} 1_{(\mathrm{C}=\mathrm{O})} \\
{ }_{(\mathrm{CH})} \mathrm{C} 1-\mathrm{H} 3 \cdots 5_{(\mathrm{C}-\mathrm{O}-\mathrm{C})} \&\left(\mathrm{CHcycl} \mathrm{C} 8-\mathrm{H} 9 \cdots \mathrm{O} 4_{(\mathrm{C}=\mathrm{O})}\right.\end{array}$ \\
\hline & $\mathrm{C}_{2}^{2}(15)$ & $\begin{array}{l}*(\mathrm{OH}) \mathrm{O} 2-\mathrm{H} 1 \cdots \mathrm{O} 4_{(\mathrm{C}=\mathrm{O})} \&(\mathrm{CH} 2) \mathrm{C} 5-\mathrm{H} 7 \cdots \mathrm{O} 1_{(\mathrm{C}=\mathrm{O})} \\
*(\mathrm{OH}) \mathrm{O} 2-\mathrm{H} 1 \cdots \mathrm{O} 4_{(\mathrm{C}=\mathrm{O})} \&(\mathrm{CH} \text { cycl }) \\
\mathrm{C} 8-\mathrm{H} 9 \cdots \mathrm{O} 4_{(\mathrm{C}=\mathrm{O})}\end{array}$ \\
\hline & $\mathrm{C}_{2}^{2}(16)$ & 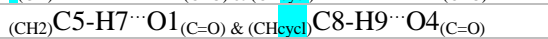 \\
\hline & $\mathrm{C}_{4}^{4}(20)$ & $\begin{array}{l}{ }^{*(\mathrm{CH})} \mathrm{C} 1-\mathrm{H} 3 \cdots \mathrm{O} 5_{(\mathrm{C}-\mathrm{O}-\mathrm{C}) \mid 2} \&\left[(\mathrm{OH}) \mathrm{O} 2-\mathrm{H} 1 \cdots \mathrm{O} 4_{(\mathrm{C}=\mathrm{O}) \mid 2}\right. \\
{ }^{*}(\mathrm{CH}) \mathrm{C} 1-\left.\mathrm{H} 3 \cdots \mathrm{O} 5_{(\mathrm{C}-\mathrm{O}-\mathrm{C})}\right|_{2} \&(\mathrm{CH} 2) \mathrm{C} 5-\mathrm{H} 7 \cdots \mathrm{O} 1_{(\mathrm{C}=\mathrm{O})}\end{array}$ \\
\hline & $\mathrm{R}_{2}^{2}(11)$ & 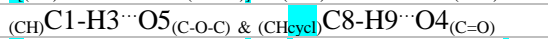 \\
\hline & $\mathrm{R}_{4}^{4}(20)$ & 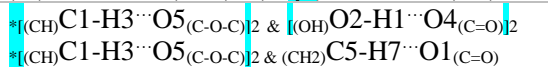 \\
\hline & & Fmoc TRYPTOPHAN derivatives \\
\hline \multirow[t]{2}{*}{ DIZNIK } & $\mathrm{C}(5)$ & $\begin{array}{l}{ }_{(\mathrm{NH})} \mathrm{N} 1-\mathrm{H} 1 \cdots \mathrm{O} 3_{(\mathrm{C}-\mathrm{O}-\mathrm{C})} \\
{ }_{(\mathrm{CH} 2)} \mathrm{C} 15-\mathrm{H} 13 \cdots \mathrm{O} 2_{(\mathrm{C}=\mathrm{O})} \\
{ }_{(\mathrm{CH})} \mathrm{C} 17-\mathrm{H} 14 \cdots \mathrm{O} 1_{(\mathrm{C}-\mathrm{O}-\mathrm{C})} \\
{ }_{(\mathrm{CH} 3)} \mathrm{C} 19-\mathrm{H} 15 \cdots \mathrm{O} 4_{(\mathrm{C}=\mathrm{O})}\end{array}$ \\
\hline & $\mathrm{C}(11)$ & $(\mathrm{CH} 2) \mathrm{C} 29-\mathrm{H} 24 \cdots \mathrm{O} 4_{(\mathrm{C}=\mathrm{O})}$ \\
\hline \multirow[t]{9}{*}{ Level 2} & $\mathrm{C}_{2}^{1}(14)$ & ${ }_{(\mathrm{CH} 3)} \mathrm{C} 19-\mathrm{H} 15 \cdots \mathrm{O} 4_{(\mathrm{C}=\mathrm{O})} \&(\mathrm{CH} 2) \mathrm{C} 29-\mathrm{H} 24 \cdots \mathrm{O} 4_{(\mathrm{C}=\mathrm{O})}$ \\
\hline & $\mathrm{C}_{2}^{2}(10)$ & $\begin{array}{l}{ }_{(\mathrm{NH})} \mathrm{N} 1-\mathrm{H} 1 \cdots \mathrm{O} 3_{(\mathrm{C}-\mathrm{O}-\mathrm{C})} \&(\mathrm{CH}) \\
{ }_{(\mathrm{CH} 3)} \mathrm{C} 19-\mathrm{H} 15 \cdots \mathrm{O} 4_{(\mathrm{C}=\mathrm{O})} \&(\mathrm{NH}) \mathrm{N} 1-\mathrm{H} 1 \cdots \mathrm{O} 1_{(\mathrm{C}-\mathrm{O}-\mathrm{C})} \\
{ }_{(\mathrm{CH} 2)} \mathrm{C} 15-\mathrm{H} 13 \cdots \mathrm{O} 2_{(\mathrm{C}-\mathrm{O})} \&(\mathrm{CH}) \mathrm{C} 17-\mathrm{H} 14 \cdots \mathrm{O} 1_{(\mathrm{C}-\mathrm{O}-\mathrm{C})}\end{array}$ \\
\hline & $\mathrm{C}_{2}^{2}(12)$ & 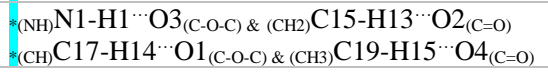 \\
\hline & $\mathrm{C}_{2}^{2}(14)$ & ${ }_{(\mathrm{NH})} \mathrm{N} 1-\mathrm{H} 1 \cdots \mathrm{O} 3_{(\mathrm{C}-\mathrm{O}-\mathrm{C})} \&(\mathrm{CH} 2) \mathrm{C} 29-\mathrm{H} 24 \cdots \mathrm{O} 4_{(\mathrm{C}=\mathrm{O})}$ \\
\hline & $\mathrm{C}_{2}^{2}(16)$ & 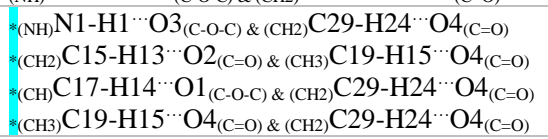 \\
\hline & $\mathrm{C}_{2}^{2}(20)$ & ${ }_{(\mathrm{CH} 2)} \mathrm{C} 15-\mathrm{H} 13 \cdots \mathrm{O} 2_{(\mathrm{C}=\mathrm{O})} \&(\mathrm{CH} 2) \mathrm{C} 29-\mathrm{H} 24 \cdots \mathrm{O} 4_{(\mathrm{C}=\mathrm{O})}$ \\
\hline & $\mathrm{R}_{2}^{2}(8)$ & $\begin{array}{l}{ }_{(\mathrm{NH})} \mathrm{N} 1-\mathrm{H} 1 \cdots \mathrm{O} 3_{(\mathrm{C}-\mathrm{O}-\mathrm{C})} \&(\mathrm{CH}) \\
{ }_{(\mathrm{NH})} \mathrm{N} 1-\mathrm{H} 1 \cdots \mathrm{O} 3_{(\mathrm{C}-\mathrm{O}-\mathrm{C})} \&(\mathrm{CH} 3) \mathrm{C} 19-\mathrm{H} 15 \cdots \mathrm{O} 1_{(\mathrm{C}-\mathrm{O}-\mathrm{C})} \\
{ }_{(\mathrm{CH} 2)} \mathrm{C} 15-\mathrm{H} 13_{(\mathrm{C}=\mathrm{O})} \mathrm{O} 2_{(\mathrm{C}=\mathrm{O})} \&(\mathrm{CH}) \mathrm{C} 17-\mathrm{H} 14 \cdots \mathrm{O} 1_{(\mathrm{C}-\mathrm{O}-\mathrm{C})}\end{array}$ \\
\hline & $\mathrm{R}_{2}^{2}(12)$ & 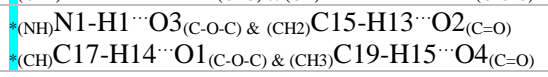 \\
\hline & $\mathrm{R}_{2}^{2}(16)$ & ${ }_{(\mathrm{CH} 2)} \mathrm{C} 15-\mathrm{H} 13 \cdots \mathrm{O} 2_{(\mathrm{C}=\mathrm{O})} \&(\mathrm{CH} 3) \mathrm{C} 19-\mathrm{H} 15 \cdots \mathrm{O} 4_{(\mathrm{C}=\mathrm{O})}$ \\
\hline
\end{tabular}


Table S8

Percentage contribution of close interactions for Fmoc-Tyr/Phe derivatives, according to HS analysis.

\begin{tabular}{|c|c|c|c|c|c|c|c|c|c|c|c|c|c|c|c|c|}
\hline & $\mathrm{H} * \mathrm{H}$ & $\mathrm{C} \cdots \mathrm{H}$ & $\mathrm{O} \cdots \mathrm{H}$ & $\mathrm{O} \cdots \mathrm{O}$ & $\mathrm{C} \cdots \mathrm{O}$ & $\mathrm{C} \cdots \mathrm{C}$ & $\mathrm{N} \cdots \mathrm{H}$ & $\mathrm{N} \cdots \mathrm{O}$ & $\mathrm{Cl}^{\prime \prime} \mathrm{C}$ & $\mathrm{Cl}{ }^{\prime \prime} \mathrm{H}$ & $\mathrm{Cl}^{\prime}{ }^{\circ} \mathrm{O}$ & $\mathrm{Br} \cdot \mathrm{C}$ & $\mathrm{Br} \cdots \mathrm{H}$ & $\mathrm{I} \cdots \mathrm{C}$ & $\mathrm{I} \cdots \mathrm{H}$ & $\mathrm{F} \cdots \mathrm{H}$ \\
\hline \multicolumn{17}{|c|}{ group $\boldsymbol{A}$} \\
\hline CAMLEK & 41.8 & 26.7 & 27.1 & & 2.6 & 1.4 & & & & & & & & & & \\
\hline OGOGIA & 42.5 & 23.6 & 16 & 0.5 & & 0.5 & 11.6 & 4.3 & & & & & & & & \\
\hline OGIYAG & 47.8 & 26.3 & 21.6 & & 1.7 & 1 & 0.6 & & & & & & & & & \\
\hline INEJEQ & 43.9 & 17.1 & 13.5 & & 0.1 & 2.1 & 0.3 & & & & & & & & & \\
\hline (1) & 60.9 & 22.2 & 15 & 1 & 0.8 & - & & & & & & & & & & \\
\hline
\end{tabular}

\begin{tabular}{llllllll}
\hline & & & & & \\
\hline OGIXOT & 48.7 & 27.4 & 20.1 & 0.3 & 2.7 & 0.7 & \\
\hline VERXUO & 53.1 & 23.6 & 19.5 & & 3 & & \\
\hline OGIXUZ & 52.7 & 25.8 & 18.9 & & 0.2 & 2 & 0.3 \\
\hline EKEWUM & 54.7 & 21.6 & 15.2 & & 3.6 & \\
\hline NUBPEH & 55.2 & 22.3 & 16.9 & 2.1 & 3.2 & \\
\hline
\end{tabular}

\begin{tabular}{llllll}
\hline \multicolumn{1}{c}{} & & & group $C$ \\
\hline MOXSUP & 57,4 & 30.2 & 11.6 & - & \\
\hline DULLAZ & 60,3 & 24,7 & 13,5 & 1,1 & \\
& & & & & \\
\hline
\end{tabular}

\begin{tabular}{llllllllllll}
\hline & \multicolumn{10}{c}{ group $D$} & \\
WATSIU01 & 32.6 & 18.2 & 26.4 & 0.3 & 0.5 & 0.5 & 1.3 & & 4.3 & 12.3 & 2.1 \\
\hline UQOGUE & 34.2 & 27.9 & 27.5 & 0.9 & 3.8 & 0.9 & 4.5 & 0.1 & & \\
\hline UQIYUQ & 52 & 28.4 & 16.8 & & 2 & 0.7 & & & & \\
\end{tabular}

\begin{tabular}{|c|c|c|c|c|c|c|c|c|c|c|c|c|}
\hline \multicolumn{13}{|c|}{ group $E$} \\
\hline XATKEL & 38.3 & 22.3 & 16.8 & 2.9 & 0.2 & 3 & 0.6 & & & & 2.5 & 12.1 \\
\hline XATJAG & 38.6 & 23 & 16.9 & 3.2 & 0.2 & 2.9 & 0.6 & & & 2.4 & 11.6 & \\
\hline XATJEK & 41.4 & 24.8 & 17.4 & 0.5 & 0.1 & 3.1 & 0.4 & 0.1 & 10.8 & 1.2 & & \\
\hline XATKIP & 44.6 & 23.8 & 16.9 & 0.3 & & 2.8 & 0.4 & & & & & 9.4 \\
\hline
\end{tabular}

\section{Table S9}

HS parameters for Fmoc-Tyr/Phe derivatives.

\begin{tabular}{|c|c|c|c|c|c|c|c|c|}
\hline CSD code & $\begin{array}{c}\text { Volume, } V \\
{\left[A^{3}\right]}\end{array}$ & $\begin{array}{c}\text { Surface } \\
\text { area, A } \\
{\left[A^{2}\right]}\end{array}$ & $A / V$ & $\begin{array}{c}\text { Globurality } \\
\text { G [-] }\end{array}$ & $\begin{array}{c}\text { Asphericity } \\
\Omega[-]\end{array}$ & $\begin{array}{c}\text { Cell } \\
\text { volume }\end{array}$ & $\begin{array}{c}\text { Occupancy } \\
\text { / unit cell } \\
{[\%]}\end{array}$ & Temp. \\
\hline & & & group $\boldsymbol{A}$ & & & & & \\
\hline CAMLEK & 494.89 & 433.30 & 0.875 & 0.698 & 0.219 & 1039.4 & 47.61 & 100 \\
\hline OGIYAG & 510.58 & 448.67 & 0.878 & 0.689 & 0.232 & 2119.4 & 24.09 & 100 \\
\hline OGOGIA & 583.85 & 476.51 & 0.816 & 0.709 & 0.122 & 2346.8 & 24.87 & 173 \\
\hline (1) & 624.88 & 492.1 & 0.78 & 0.718 & 0.128 & 2531.9 & 24.68 & 100 \\
\hline \multirow[t]{2}{*}{ INEJEQ } & 824.98 & 664.44 & 0.80 & 0.640 & 0.166 & 1327.8 & 62.12 & 160 \\
\hline & & & group $\boldsymbol{B}$ & & & & & \\
\hline VERXUO & 457.56 & 414.76 & 0.90 & 0.692 & 0.198 & 1256.7 & 36.41 & 100 \\
\hline OGIXUZ & 469.73 & 421.58 & 0.89 & 0.693 & 0.185 & 1176.2 & 39.93 & 100 \\
\hline OGIXOT & 470.11 & 423.37 & 0.90 & 0.691 & 0.195 & 993.8 & 47.30 & 100 \\
\hline EKEWUM & 479.13 & 413.66 & 0.86 & 0.686 & 0.242 & 2055.2 & 23.31 & 173 \\
\hline \multirow[t]{2}{*}{ NUBPEH } & 684.44 & 543.95 & 0.79 & 0.690 & 0.118 & 5662 & 12.09 & 100 \\
\hline & & & group C & & & & & \\
\hline DULLAZ & 567.62 & 460.54 & 0.81 & 0.727 & 0.128 & 2372.2 & 23.92 & 110 \\
\hline \multirow[t]{2}{*}{ MOXSUP } & 658.19 & 530.24 & 0.80 & 0.690 & 0.140 & 4995.9 & 13.17 & 90 \\
\hline & & & group $\boldsymbol{D}$ & & & & & \\
\hline UQIYUQ & 489.42 & 435.06 & 0.88 & 0.690 & 0.265 & 2083.8 & 23.48 & 100 \\
\hline UQOGUE & 496.97 & 441.70 & 0.88 & 0.687 & 0.261 & 2161.2 & 22.99 & 100 \\
\hline \multirow[t]{2}{*}{ WATSIU01 } & 517.56 & 426.61 & 0.82 & 0.731 & 0.116 & 2929.5 & 17.66 & 100 \\
\hline & & & group $\boldsymbol{E}$ & & & & & \\
\hline XАТКIP & 468.90 & 420.43 & 0.89 & 0.694 & 0.183 & 2614 & 17.93 & 100 \\
\hline XАTJAG & 494.48 & 409.14 & 0.82 & 0.739 & 0.133 & 3238.4 & 15.27 & 100 \\
\hline XATKEL & 500.69 & 413.45 & 0.82 & 0.738 & 0.123 & 3319.4 & 15.08 & 100 \\
\hline
\end{tabular}




\begin{tabular}{lllllllll}
\hline XATJEK & 506.94 & 451.24 & 0.89 & 0.681 & 0.184 & 1035.3 & 48.96 & 100 \\
\hline
\end{tabular}

*Occupancy per unit cell - Hirshfeld surface volume / cell volume $\times 100 \%$

Table S10

Percentage contributions of close interactions for other Fmoc-AAs (above $1 \%$ ).

\begin{tabular}{|c|c|c|c|c|c|c|c|c|c|c|c|}
\hline CSD code & $\mathrm{H}^{\cdots \cdots} \mathrm{H}$ & $\mathrm{C} \cdots \mathrm{H}$ & $\mathrm{O} \cdots \mathrm{H}$ & $\mathrm{O} \cdots \mathrm{O}$ & $\mathrm{C} \cdots \mathrm{O}$ & $\mathrm{C} \cdots \mathrm{C}$ & $\mathrm{N} \cdots \mathrm{H}$ & $\mathrm{F}^{\cdots} \mathrm{H}$ & $\mathrm{F}^{\cdots} \mathrm{F}$ & $\mathrm{F}^{\cdots} \mathrm{C}$ & $\mathrm{F}^{\cdots} \mathrm{O}$ \\
\hline \multicolumn{12}{|c|}{ Fmoc-GLY CINE derivatives } \\
\hline NOVTOJ & 54.5 & 17.9 & 23.8 & & 0.3 & 3.3 & 0.1 & & & & \\
\hline XAVYIE & 63.2 & 14.3 & 22 & & & & & & & & \\
\hline VERQER & 26.1 & 15.5 & 16.7 & & 1.8 & 1.6 & & 22.8 & 7.5 & 7.2 & \\
\hline VERQIV & 40.1 & 28.8 & 19.7 & & 1.5 & 1.7 & & 6 & & 1 & \\
\hline VERQOB & 26.4 & 18 & 15.7 & & & & & 28 & 4.5 & 4.7 & 1.1 \\
\hline VERXOI & 46.3 & 29.8 & 19.4 & 1 & 1.5 & 1.4 & & & & & \\
\hline VERXIC & 32.5 & 24 & 33.9 & 1.4 & 4.2 & 1.7 & 1.5 & & & & \\
\hline \multicolumn{12}{|c|}{ Fmoc-ALANINE derivatives } \\
\hline ADAGUK & 48.7 & 29 & 17.5 & & & 2.7 & & & & & \\
\hline CUWKIO01 & 49.1 & 26.7 & 22 & & 1.1 & & & & & & \\
\hline CUWKOU01 & 58.2 & 25.3 & 16 & & & & & & & & \\
\hline
\end{tabular}

\begin{tabular}{lllllll}
\hline & & \multicolumn{5}{c}{ Fmoc-SERINE derivatives } \\
\hline ADAGOE & 38.3 & 27.8 & 29.3 & 2.7 & 1.2 \\
MOHCIW & 59.9 & 16.3 & 20.6 & \multicolumn{4}{c}{2.5} \\
& & & & & \\
\hline
\end{tabular}

\begin{tabular}{ll}
\hline & Fmoc CYSTEINE derivative \\
\hline EJEWUL & No $3 D$ coordinates
\end{tabular}

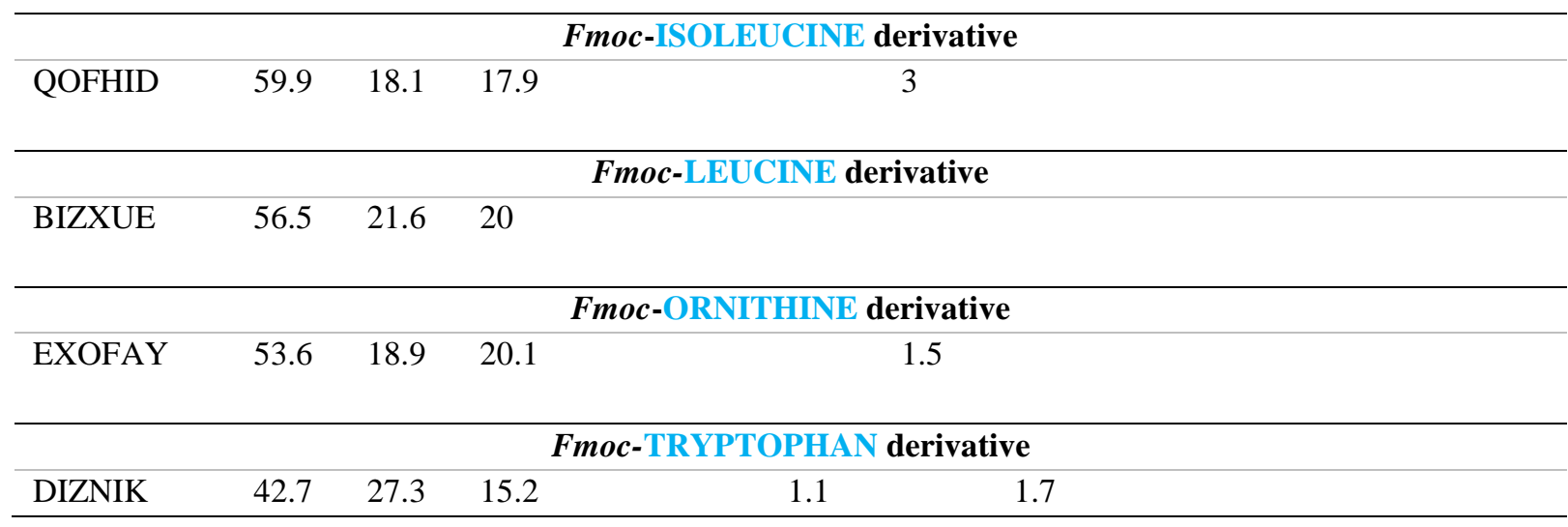

\section{Table S11}

$\pi \cdots \pi$ motifs in Fmoc-Tyr/Phe derivatives.

\begin{tabular}{|c|c|c|c|c|c|}
\hline & $\begin{array}{c}\text { [ratio } \mathrm{C} \cdots \mathrm{H} / \\
\mathrm{C} \cdots \mathrm{C}]\end{array}$ & Motif & & $\begin{array}{c}\text { [ratio } \mathrm{C}^{\cdots} \mathrm{H} / \\
\mathrm{C} \cdots \mathrm{C}]\end{array}$ & Motif \\
\hline CAMLEK & 19.1 & herringbone & NOVTOJ & 5.42 & herringbone \\
\hline OGOGIA & 47.2 & herringbone & XAVYIE & - & - \\
\hline OGIYAG & 26.3 & herringbone & VERQER & 9.7 & herringbone \\
\hline INEJEQ & 8.1 & herringbone & VERQIW & 16.9 & herringbone \\
\hline (1) & - & - & VERQOB & - & \\
\hline
\end{tabular}




\begin{tabular}{lrlcrl}
\hline OGIXOT & 10.1 & herringbone & VERXOI & 21.3 & herringbone \\
VERXUO & 7.9 & herringbone & VERXIC & 14.1 & herringbone \\
OGIXUZ & 12.9 & herringbone & ADAGUK & 10.7 & herringbone \\
EKEWUM & 6 & herringbone & CUWKIO01 & - & \\
NUBPEH & 6.9 & herringbone & CUWKOU01 & - & \\
MOXSUP & - & - & ADAGOE & - & \\
DULLAZ & 22.4 & herringbone & MOHCIW & 6.5 & herringbone \\
WATSIU01 & 36.4 & herringbone & EJEWUL & & \\
UQOGUE & 31 & herringbone & QOFHID & 6 & herringbone \\
UQIYUQ & 40.6 & herringbone & BIZXUE & - & - \\
XATKEL & 7.4 & herringbone & EXOFAY & 12.6 & herringbone \\
XATJAG & 7.9 & herringbone & DIZNIK & - & - \\
XATJEK & 8 & herringbone & & & \\
XATKIP & 8.5 & herringbone & & & \\
\hline
\end{tabular}

